\title{
Harmonic analysis of a class of reproducing kernel Hilbert spaces arising from groups
}

\author{
Palle Jorgensen, Steen Pedersen, and Feng Tian
}

Author address:

(Palle E.T. Jorgensen) Department of Mathematics, The UniverSITY OF IOWA, IOWA CITY, IA 52242-1419, U.S.A.

E-mail address: palle-jorgensen@uiowa.edu

$U R L:$ http://www.math.uiowa.edu/ jorgen/

(Steen Pedersen) Department of Mathematics, Wright State UniVERsity, Dayton, OH 45435, U.S.A.

E-mail address: steen@math.wright.edu

URL: http://www.wright.edu/ steen. pedersen/

(Feng Tian) Department of Mathematics, Wright State University, DAYTON, OH 45435, U.S.A.

E-mail address: feng.tian@wright.edu

URL: http://www. wright.edu/ feng.tian/ 


\section{Contents}

Chapter 1. Introduction 5

1.1. Two Extension Problems 6

1.2. Stochastic Processes 6

1.3. Earlier Papers $\quad 8$

1.4. Organization 8

1.5. Notation and Preliminaries $\quad 10$

1.6. Overview of Applications of RKHSs 11

Chapter 2. Extensions of Continuous Positive Definite Functions 12

2.1. Enlarging the Hilbert Space 12

2.2. $\operatorname{Ext}_{1}(F)$ and $\operatorname{Ext}_{2}(F) \quad 15$

$\begin{array}{ll}\text { 2.3. Preliminaries } & 17\end{array}$

2.4. The Case of Locally Compact Abelian Groups 23

2.5. The Case of $G=\mathbb{R}^{n} \quad 26$

2.6. Lie Groups 30

2.7. The Circle Group $\mathbb{T} \quad 33$

2.8. Example: $e^{i 2 \pi x} \quad 34$

2.9. Example: $e^{-a|x|} \quad 35$

2.10. The Example $e^{-|x|}$ in $(-a, a) \quad 37$

2.11. Discussion 43

Chapter 3. Type I v.s. Type II Extensions 45

3.1. Polya Extensions $\quad 45$

3.2. The Example 3.1.5 53

Chapter 4. The Computation of $\operatorname{Ext}_{1}(F) \quad 56$

4.1. Mercer Operators, and Bessel Frames 56

4.2. Positive Definite Functions, Green's Functions, and Boundary $\quad 77$

4.3. The RKHSs for the Two Examples $F_{2}$ and $F_{3}$ in Table 3.1.1 82

4.4. Higher Dimensions 94

Chapter 5. Comparing Different RKHSs $\mathscr{H}_{F}$ and $\mathscr{H}_{K} \quad 96$

5.1. Applications $\quad 99$

5.2. Radially Symmetric Positive Definite Functions 101

5.3. Connecting $F$ and $\bar{F}$ When $F$ is a Positive Definite Function 102

5.4. The Imaginary Part of a Positive Definite Function 104

$\begin{array}{lll}\text { Chapter 6. Convolution Products } & 110\end{array}$

Chapter 7. Models for Operator Extensions 112

7.1. Model for Restrictions of Continuous p.d. Functions on $\mathbb{R}$ 
7.2. A Model of ALL Deficiency Index- $(1,1)$ Operators 116

7.3. The Case of Indices $(d, d)$ where $d>1 \quad 120$

7.4. Spectral Representation of Index $(1,1)$ Hermitian Operators 122

Chapter 8. Open Questions 126

8.1. From Restriction Operator to Restriction of p.d. Function 126

8.2. The Splitting $\mathscr{H}_{F}=\mathscr{H}_{F}^{(\text {atom })} \oplus \mathscr{H}_{F}^{(a c)} \oplus \mathscr{H}_{F}^{(\text {sing })} \quad 126$

8.3. The Case of $G=\mathbb{R}^{1} \quad 127$

8.4. The Extreme Points of Ext $(F)$ and $\Im\{F\} \quad 128$

$\begin{array}{ll}\text { List of Figures } & 129\end{array}$

List of Tables 131

List of Symbols 132

Index 133

$\begin{array}{ll}\text { Bibliography } & 135\end{array}$ 


\begin{abstract}
We study two extension problems, and their interconnections: (i) extension of positive definite (p.d.) continuous functions defined on subsets in locally compact groups $G$; and (ii) (in case of Lie groups $G$ ) representations of the associated Lie algebras $L a(G)$, i.e., representations of $L a(G)$ by unbounded skew-Hermitian operators acting in a reproducing kernel Hilbert space $\mathscr{H}_{F}$ (RKHS). Our analysis is non-trivial even if $G=\mathbb{R}^{n}$, and even if $n=1$. If $G=\mathbb{R}^{n}$, (ii), we are concerned with finding systems of strongly commuting selfadjoint operators $\left\{T_{i}\right\}$ extending a system of commuting Hermitian operators with common dense domain in $\mathscr{H}_{F}$.

Specifically, we consider partially defined positive definite (p.d.) continuous functions $F$ on a fixed group. From $F$ we then build a reproducing kernel Hilbert space $\mathscr{H}_{F}$, and the operator extension problem is concerned with operators acting in $\mathscr{H}_{F}$, and with unitary representations of $G$ acting on $\mathscr{H}_{F}$. Our emphasis is on the interplay between the two problems, and on the harmonic analysis of our RKHSs $\mathscr{H}_{F}$.

In the cases of $G=\mathbb{R}^{n}$, and $G=\mathbb{T}^{n}=\mathbb{R}^{n} / \mathbb{Z}^{n}$, and generally for locally compact Abelian groups, we establish a new Fourier duality theory; including for $G=\mathbb{R}^{n}$ a time/frequency duality, where the extension questions (i) are in time domain, and extensions from (ii) in frequency domain. Specializing to $n=1$, we arrive of a spectral theoretic characterization of all skew-Hermitian operators with dense domain in a separable Hilbert space, having deficiency-indices $(1,1)$.

Our general results include non-compact and non-Abelian Lie groups, where the study of unitary representations in $\mathscr{H}_{F}$ is subtle.

Received by the editor $01 / 19 / 14$.

2000 Mathematics Subject Classification. Primary 47L60, 46N30, 46N50, 42C15, 65R10; Secondary 46N20, 22E70, 31A15, 58J65, 81S25.

Key words and phrases. Unbounded operators, deficiency-indices, Hilbert space, reproducing kernels, boundary values, unitary one-parameter group, convex, harmonic decompositions, stochastic processes, representations of Lie groups, potentials, quantum measurement, renormalization, partial differential operators, rank-one perturbation, Friedrichs extension, Green's function.

The co-authors thank the following for enlightening discussions: Professors Sergii Bezuglyi, Dorin Dutkay, Paul Muhly, Myung-Sin Song, Wayne Polyzou, Gestur Olafsson, Robert Niedzialomski, and members in the Math Physics seminar at the University of Iowa.
\end{abstract}




\section{CHAPTER 1}

\section{Introduction}

In this Memoir, we study two extension problems, and their interconnections. The first class of extension problems concerns (i) positive definite (p.d.) continuous functions on Lie groups $G$, and the second deals with (ii) Lie algebras of unbounded skew-Hermitian operators in a certain family of reproducing kernel Hilbert spaces (RKHS). Our analysis is non-trivial even if $G=\mathbb{R}^{n}$, and even if $n=1$. If $G=\mathbb{R}^{n}$, we are concerned in (ii) with the study of systems of $n$ skew-Hermitian operators $\left\{S_{i}\right\}$ on a common dense domain in Hilbert space, and in deciding whether it is possible to find a corresponding system of strongly commuting selfadjoint operators $\left\{T_{i}\right\}$ such that, for each value of $i$, the operator $T_{i}$ extends $S_{i}$.

The version of this for non-commutative Lie groups $G$ will be stated in the language of unitary representations of $G$, and corresponding representations of the Lie algebra $L a(G)$ by skew-Hermitian unbounded operators.

In summary, for (i) we are concerned with partially defined positive definite (p.d.) continuous functions $F$ on a Lie group; i.e., at the outset, such a function $F$ will only be defined on a connected proper subset in $G$. From this partially defined p.d. function $F$ we then build a reproducing kernel Hilbert space $\mathscr{H}_{F}$, and the operator extension problem (ii) is concerned with operators acting on $\mathscr{H}_{F}$, as well as with unitary representations of $G$ acting on $\mathscr{H}_{F}$. If the Lie group $G$ is not simply connected, this adds a complication, and we are then making use of the associated simply connected covering group. For an overview of highpoints in our Memoir, see sections 1.4 and 1.6 below.

By a theorem of Kolmogorov, every Hilbert space may be realized as a (Gaussian) reproducing kernel Hilbert space (RKHS), see e.g., [PS75, IM65, SNFBK10]. Since this setting is too general for many applications, it is useful to restrict the very general framework for RKHSs to concrete cases in the study of particular spectral theoretic problems; positive definite functions on groups is a case in point. Such specific issues arise in physics (see e.g., [Fal74, Jor07]) where one is faced with extending positive definite functions $F$ which are only defined on a subset of a given group, say $G$. For a given such $F$, we introduce an associated RKHS $\mathscr{H}_{F}$ constructed directly from the given, partially defined, p.d. function $F$.

The axioms of quantum physics (see e.g., [BM13, OH13, KS02, CRKS79, ARR13, Fan10, Maa10, Par09] for relevant recent papers), are based on Hilbert space, and selfadjoint operators.

A brief sketch: A quantum mechanical observable is a Hermitian (selfadjoint) linear operator mapping a Hilbert space, the space of states, into itself. The values obtained in a physical measurement are in general described by a probability distribution; and the distribution represents a suitable "average" (or "expectation") in a measurement of values of some quantum observable in a state of some prepared system. The states are (up to phase) unit vectors in the Hilbert space, and a 
measurement corresponds to a probability distribution (derived from a projectionvalued spectral measure). The particular probability distribution used depends on both the state and the selfadjoint operator. The associated spectral type may be continuous (such as position and momentum; both unbounded) or discrete (such as spin); this depends on the physical quantity being measured.

Since the spectral theorem serves as the central tool in our study of measurements, we must be precise about the distinction between linear operators with dense domain which are only Hermitian as opposed to selfadjoint. This distinction is accounted for by von Neumann's theory of deficiency indices [AG93, DS88].

(Starting with [vN32a, vN32c, vN32b], J. von Neumann and M. Stone did pioneering work in the 1930s on spectral theory for unbounded operators in Hilbert space; much of it in private correspondence. The first named author has from conversations with M. Stone, that the notions "deficiency-index," and "deficiency space" are due to them; suggested by MS to vN as means of translating more classical notions of "boundary values" into rigorous tools in abstract Hilbert space: closed subspaces, projections, and dimension count.)

\subsection{Two Extension Problems}

While each of the two extension problems has received a considerable amount of attention in the literature, our emphasis here will be the interplay between the two problems: Our aim is a duality theory; and, in the case $G=\mathbb{R}^{n}$, and $G=\mathbb{T}^{n}=$ $\mathbb{R}^{n} / \mathbb{Z}^{n}$, we will state our theorems in the language of Fourier duality of abelian groups: With the time frequency duality formulation of Fourier duality for $G=\mathbb{R}^{n}$ we have that both the time domain and the frequency domain constitute a copy of $\mathbb{R}^{n}$. We then arrive at a setup such that our extension questions (i) are in time domain, and extensions from (ii) are in frequency domain. Moreover we show that each of the extensions from (i) has a variant in (ii). Specializing to $n=1$, we arrive of a spectral theoretic characterization of all skew-Hermitian operators with dense domain in a separable Hilbert space, having deficiency-indices $(1,1)$.

A systematic study of densely defined Hermitian operators with deficiency indices $(1,1)$, and later $(d, d)$, was initiated by M. Krein [Kre46], and is also part of de Branges' model theory; see [dB68, dBR66]. The direct connection between this theme and the problem of extending continuous positive definite (p.d.) functions $F$ when they are only defined on a fixed open subset to $\mathbb{R}^{n}$ was one of our motivations. One desires continuous p.d. extensions to $\mathbb{R}^{n}$.

If $F$ is given, we denote the set of such extensions $\operatorname{Ext}(F)$. If $n=1, \operatorname{Ext}(F)$ is always non-empty, but for $n=2$, Rudin gave examples in [Rud70, Rud63] when $\operatorname{Ext}(F)$ may be empty. Here we extend these results, and we also cover a number of classes of positive definite functions on locally compact groups in general; so cases when $\mathbb{R}^{n}$ is replaced with other groups, both Abelian and non-abelian.

Our results in the framework of locally compact Abelian groups are more complete than their counterparts for non-Abelian Lie groups, one reason is the availability of Bochner's duality theorem for locally compact Abelian groups; - not available for non-Abelian Lie groups.

\subsection{Stochastic Processes}

The interest in positive definite functions has at least three roots: (i) Fourier analysis, and harmonic analysis more generally, including the non-commutative 
variant where we study unitary representations of groups; (ii) optimization and approximation problems, involving for example spline approximations as envisioned by I. Schöenberg; and (iii) the study of stochastic (random) processes.

Below, we sketch a few details regarding (iii). A stochastic process is an indexed family of random variables based on a fixed probability space; in our present analysis, our processes will be indexed by some group $G$; for example $G=\mathbb{R}$, or $G=\mathbb{Z}$ correspond to processes indexed by real time, respectively discrete time. A main tool in the analysis of stochastic processes is an associated covariance function, see (1.2.1).

A process $\left\{X_{g} \mid g \in G\right\}$ is called Gaussian if each random variable $X_{g}$ is Gaussian, i.e., its distribution is Gaussian. For Gaussian processes we only need two moments. So if we normalize, setting the mean equal to 0 , then the process is determined by the covariance function. In general the covariance function is a function on $G \times G$, or on a subset, but if the process is stationary, the covariance function will in fact be a positive definite function defined on $G$, or a subset of $G$. We will be using three stochastic processes in the Memoir, Brownian motion, Brownian Bridge, and the Ornstein-Uhlenbeck process, all Gaussian, or Ito integrals.

We outline a brief sketch of these facts below.

Let $G$ be a locally compact group, and let $(\Omega, \mathscr{F}, \mathbb{P})$ be a probability space, $\mathscr{F}$ a sigma-algebra, and $\mathbb{P}$ a probability measure defined on $\mathscr{F}$. A stochastic $L^{2}$-process is a system of random variables $\left\{X_{g}\right\}_{g \in G}, X_{g} \in L^{2}(\Omega, \mathscr{F}, \mathbb{P})$. The covariance function $c_{X}$ of the process is the function $G \times G \rightarrow \mathbb{C}$ given by

$$
c_{X}\left(g_{1}, g_{2}\right)=\mathbb{E}\left(\bar{X}_{g_{1}} X_{g_{2}}\right), \forall\left(g_{1}, g_{2}\right) \in G \times G .
$$

To simplify will assume that the mean $\mathbb{E}\left(X_{g}\right)=\int_{\Omega} X_{g} d \mathbb{P}(\omega)=0$ for all $g \in G$.

We say that $\left(X_{g}\right)$ is stationary iff

$$
c_{X}\left(h g_{1}, h g_{2}\right)=c_{X}\left(g_{1}, g_{2}\right), \forall h \in G .
$$

In this case $c_{X}$ is a function of $g_{1}^{-1} g_{2}$, i.e.,

$$
\mathbb{E}\left(X_{g_{1}}, X_{g_{2}}\right)=c_{X}\left(g_{1}^{-1} g_{2}\right), \forall g_{1}, g_{2} \in G .
$$

(Just take $h=g_{1}^{-1}$ in (1.2.2).)

We now recall the following theorem of Kolmogorov (see [PS75]). One direction is easy, and the other is the deep part:

Definition 1.2.1. A function $c$ defined on a subset of $G$ is said to be positive definite iff

$$
\sum_{i} \sum_{j} \overline{\lambda_{i}} \lambda_{j} c\left(g_{i}^{-1} g_{j}\right) \geq 0
$$

for all finite summation, where $x_{i} \in \mathbb{C}$ and $g_{i}^{-1} g_{j}$ in the domain of $c$.

TheOREM 1.2.2 (Kolmogorov). A function $c: G \rightarrow \mathbb{C}$ is positive definite if and only if there is a stationary Gaussian process $(\Omega, \mathscr{F}, \mathbb{P}, X)$ with mean zero, such that $c=c_{X}$.

Proof. To stress the idea, we include the easy part of the theorem, and we refer to $[\mathbf{P S 7 5}]$ for the non-trivial direction: 
Let $\lambda_{1}, \lambda_{2}, \ldots, \lambda_{n} \in \mathbb{C}$, and $\left\{g_{i}\right\}_{i=1}^{N} \subset G$, then for all finite summations, we have:

$$
\sum_{i} \sum_{j} \overline{\lambda_{i}} \lambda_{j} c_{X}\left(g_{i}^{-1} g_{j}\right)=\mathbb{E}\left(\left|\sum_{i=1}^{N} \lambda_{i} X_{g_{i}}\right|^{2}\right) \geq 0 .
$$

\subsection{Earlier Papers}

Below we mention some earlier papers dealing with one or the other of the two extension problems (i) or (ii) in section 1.1. To begin with, there is a rich literature on (i), a little on (ii), but comparatively much less is known about their interconnections.

As for positive definite functions, their use and applications are extensive and includes such areas as stochastic processes, see e.g., [JP13a, AJSV13, JP12, AJ12]; harmonic analysis (see [BCR84, JÓ00, JÓ98]), and the references there); potential theory [Fug74, KL14]; operators in Hilbert space $\left[\mathrm{ADL}^{+}\right.$10, Alp92, AD86]; and spectral theory [AH13, Nus75, Dev72, Dev59]. We stress that the literature is vast, and the above list is only a small sample.

Extensions of positive definite (p.d.) continuous functions defined on subsets of Lie groups $G$ was studied in [Jor91a]. In our present analysis of connections between extensions of positive definite (p.d.) continuous functions and extension questions for associated operators in Hilbert space, we will be making use of tools from spectral theory, and from the theory of reproducing kernel-Hilbert spaces, such as can be found in e.g., [Nel69, Jør81, ABDdS93, Aro50].

There is a different kind of notion of positivity involving reflections, restrictions, and extensions. It comes up in physics and in stochastic processes, and is somewhat related to our present theme. While they have several names, "refection positivity" is a popular term.

In broad terms, the issue is about realizing geometric reflections as "conjugations" in Hilbert space. When the program is successful, for a given unitary representation $U$ of a Lie group $G$, for example $G=\mathbb{R}$, it is possible to renormalize the Hilbert space on which $U$ is acting.

Now the Bochner transform $F$ of a probability measure (e.g., the distribution of a stochastic process) which further satisfies refection positivity, has two positivity properties: one (i) because $F$ is the transform of a positive measure, so $F$ is positive definite; and in addition the other, (ii) because of refection symmetry. We have not followed up below with structural characterizations of this family of positive definite functions, but readers interested in the theme, will find details in [JÓ00, JÓ98, Arv86, OS73], and in the references given there.

\subsection{Organization}

The Memoir is organized around the following themes, some involving dichotomies; e.g.,

(1) abelian vs non-abelian;

(2) simply connected vs quotients;

(3) spectral theoretic vs geometric;

(4) extending of positive definite functions vs extensions of systems of operators; and 
(5) existence vs computation and classification.

Item (1) refers to the group $G$ under consideration. In order to get started, we will need $G$ to be locally compact so it comes with Haar measure, but it may be non-abelian. It may be a Lie group, or it may be non-locally Euclidean. In the other end of this dichotomy, we look at $G=\mathbb{R}$, the real line. In all cases, in the study of the themes from (1) it is important whether the group is simply connected or not.

In order to quickly get to concrete examples, we begin the real line $G=\mathbb{R}$, and $G=\mathbb{R}^{n}, n>1$ (section 2.5); and the circle group, $G=\mathbb{T}=\mathbb{R} / \mathbb{Z}$ (section 2.7).

Of the other groups, we offer a systematic treatment of the classes when $G$ is locally compact Abelian (section 2.4), and the case of Lie groups (section 2.6).

We note that the subdivision into classes of groups is necessary as the theorems we prove in the case of $G=\mathbb{R}$ have a lot more specificity than their counterparts do, for the more general classes of groups. One reason for this is that our harmonic analysis relies on unitary representations, and the non-commutative theory for unitary representations is much more subtle than is the Abelian counterpart.

Taking a choice of group $G$ as our starting point, we then study continuous positive definite functions $F$ defined on certain subsets in $G$. In the case of $G=\mathbb{R}$, our choice of subset will be a finite open interval centered at $x=0$.

Our next step is to introduce a reproducing kernel Hilbert space (RKHS) $\mathscr{H}_{F}$ that captures the properties of the given p.d. function $F$. The nature and the harmonic analysis of this particular RKHS are of independent interest; see sections $1.6,2.1,2.5$, and 4.1 .

In section 4.1, we study a certain trace class integral operator (the Mercer operator). A mercer operator $T_{F}$ is naturally associated to a given a continuous and positive definite function $F$ defined on the open interval $(-1,1)$. We use $T_{F}$ in order to identify natural Bessel frame in the RKHS $\mathscr{H}_{F}$ corresponding to $F$. We then introduce a notion of Shannon sampling of finite Borel measures on $\mathbb{R}$, sampling from integer points in $\mathbb{R}$. In Corollary 4.1 .16 we then use this to give a necessary and sufficient condition for a given finite Borel measure $\mu$ to fall in the convex set $\operatorname{Ext}(F)$ : The measures in $\operatorname{Ext}(F)$ are precisely those whose Shannon sampling recover the given p.d. function $F$ on the interval $(-1,1)$.

The questions we address (in the general case for $G$ ) are as follows:

(a) What (if any) are the continuous positive definite functions on $G$ which extend $F$ ? (See section 2.1, section 2.10, chapter 3, and chapter 4.) Denoting the set of these extensions $\operatorname{Ext}(F)$, then $\operatorname{Ext}(F)$ is a compact convex set. Our next questions are:

(b) What are the parameters for $\operatorname{Ext}(F)$ ? (See section 2.10, chapter 3, and chapter 8.)

And (c) How can we understand $\operatorname{Ext}(F)$ from a (generally non-commutative) extension problem for operators in $\mathscr{H}_{F}$ ? (See especially section 4.1.)

We are further concerned with (d) applications to scattering theory (e.g., Theorem 2.5.1), and to commutative and non-commutative harmonic analysis.

The unbounded operators we consider are defined naturally from given p.d. function $F$, and they have a common dense domain in the RKHS $\mathscr{H}_{F}$. In studying possible selfadjoint operator extensions in $\mathscr{H}_{F}$ we make use of von Neumann's theory of deficiency indices. 
For concrete cases, (e) we must then find the deficiency indices; they must be equal, but whether they are $(0,0),(1,1),(d, d), d>1$, is of great significance to the answers to the questions from (a)-(d).

Finally, (f): What is the relevance of the solutions in (a) and (b) for the theory of operators in Hilbert space and their harmonic (and spectral) analysis? (Sections $2.5,7.2,7.3$, and chapter 8.)

\subsection{Notation and Preliminaries}

In our theorems and proofs, we shall make use of reproducing kernel Hilbert spaces (RKHS), but the particular RKHSs we need here will have additional properties (as compared to a general framework); which allow us to give explicit formulas for our solutions. In a general setup, reproducing kernel Hilbert spaces were pioneered by Aronszajn in the 1950s [Aro50]; and subsequently they have been used in a host of applications; e.g., [SZ09, SZ07].

The key idea of Aronszajn is that a RKHS is a Hilbert space $\mathscr{H}_{K}$ of functions $f$ on a set such that the values $f(x)$ are "reproduced" from $f$ in $\mathscr{H}_{K}$ and a vector $K_{x}$ in RKHS, in such a way that the inner product $\left\langle K_{x}, K_{y}\right\rangle=: K(x, y)$ is a positive definite kernel. Our present setting is more restrictive in two ways: $(i)$ we study groups $G$, and translation-invariant kernels, and $(i i)$ we further impose continuity. By "translation" we mean relative to the operation in the particular group under discussion. Our presentation below begins with the special case when $G$ is the circle group $\mathbb{T}(:=\mathbb{R} / \mathbb{Z})$, or the real line $\mathbb{R}$.

1.5.1. Reproducing Kernel Hilbert spaces. For simplicity we focus on the case $G=\mathbb{R}$, indicating the changes needed for $G=\mathbb{T}$. Modifications, if any, necessitated by considering other groups $G$ will be described in the body of the Memoir.

Definition 1.5.1. Fix $0<a$, let $\Omega$ be an open interval of length $a$, then $\Omega-\Omega=(-a, a)$. A function

$$
F: \Omega-\Omega \rightarrow \mathbb{C}
$$

be continuous, bounded, and defined on $\Omega-\Omega . F$ is positive definite (p.d.) if

$$
\sum_{i} \sum_{j} \overline{c_{i}} c_{j} F\left(x_{i}-x_{j}\right) \geq 0, \text { for all finite sums with } c_{i} \in \mathbb{C}, \text { and all } x_{i} \in \Omega \text {. }
$$

Hence, $F$ is positive definite iff the $N \times N$ matrices $\left(F\left(x_{i}-x_{j}\right)\right)_{i, j=1}^{N}$ are positive definite for all $x_{1}, \ldots, x_{N}$ in $\Omega$ and all $N$.

LEMMA 1.5.2. F is p.d. iff

$$
\int_{\Omega} \int_{\Omega} \overline{\varphi(x)} \varphi(y) F(x-y) d x d y \geq 0 \text { for all } \varphi \in C_{c}^{\infty}(\Omega) .
$$

Proof. Standard.

Consider a continuous positive definite function so $F$ is defined on $\Omega-\Omega$. Let $\mathscr{H}_{F}$ be the reproducing kernel Hilbert space (RKHS), which is the completion of

$$
\sum_{\text {finite }} c_{j} F\left(\cdot-x_{j}\right): c_{j} \in \mathbb{C}
$$


with respect to the inner product

$$
\langle F(\cdot-x), F(\cdot-y)\rangle_{\mathscr{H}_{F}}=F(x-y), \forall x, y \in \Omega
$$

and

$$
\left\langle\sum_{i} c_{i} F\left(\cdot-x_{i}\right), \sum_{j} d_{j} F\left(\cdot-y_{j}\right)\right\rangle_{\mathscr{H}_{F}}=\sum_{i} \sum_{j} \overline{c_{i}} d_{j} F\left(x_{i}-y_{j}\right),
$$

Throughout, we use the convention that the inner product is conjugate linear in the first variable, and linear in the second variable. When more than one inner product is used, subscripts will make reference to the Hilbert space.

LEmma 1.5.3. The RKHS, $\mathscr{H}_{F}$, is the Hilbert completion of the functions

$$
F_{\varphi}(x)=\int_{\Omega} \varphi(y) F(x-y) d y, \forall \varphi \in C_{c}^{\infty}(\Omega), x \in \Omega
$$

with respect to the inner product

$$
\left\langle F_{\varphi}, F_{\psi}\right\rangle_{\mathscr{H}_{F}}=\int_{\Omega} \int_{\Omega} \overline{\varphi(x)} \psi(y) F(x-y) d x d y, \forall \varphi, \psi \in C_{c}^{\infty}(\Omega) .
$$

In particular,

$$
\left\|F_{\varphi}\right\|_{\mathscr{H}_{F}}^{2}=\int_{\Omega} \int_{\Omega} \overline{\varphi(x)} \varphi(y) F(x-y) d x d y, \forall \varphi \in C_{c}^{\infty}(\Omega)
$$

and

$$
\left\langle F_{\varphi}, F_{\psi}\right\rangle_{\mathscr{H}_{F}}=\int_{\Omega} \overline{\varphi(x)} F_{\psi}(x) d x, \forall \phi, \psi \in C_{c}^{\infty}(\Omega) .
$$

\subsection{Overview of Applications of RKHSs}

Subsequently, we shall be revisiting a number of specific instances of these Reproducing kernel Hilbert spaces (RKHSs). Our use of them ranges from the most general case, when a continuous positive definite (p.d.) function $F$ is defined on an open subset of a locally compact group; the RKHS will be denoted $\mathscr{H}_{F}$. However, we stress that the associated RKHS will depend on both the function $F$, and on the subset of $G$ where $F$ is defined; hence on occasion, to be specific about the subset, we shall index the RKHS by the pair $(\Omega, F)$. If the choice of subset is implicit in the context, we shall write simply $\mathscr{H}_{F}$. Depending on the context, a particular RKHS typically will have any number of concrete, hands-on realizations, allowing us thereby to remove the otherwise obtuse abstraction entailed in its initial definition.

A glance at the Table of Contents indicates a large variety of the classes of groups, and locally defined p.d. functions we consider, the subsets. In each case, both the specific continuous, locally defined p.d. function considered, and its domain are important. Each of the separate cases has definite applications. The most explicit computations work best in the case when $G=\mathbb{R}$; and we offer a number of applications in three areas: applications to stochastic processes (sect 3.1-4.4), to harmonic analysis (sections 7.1-7.4), and to operator/spectral theory (section 4.1.). 


\section{CHAPTER 2}

\section{Extensions of Continuous Positive Definite Functions}

Our main theme is the interconnection between (i) the study of extensions of locally defined continuous and positive definite (p.d.) functions $F$ on groups on the one hand, and, on the other, (ii) the question of extensions for an associated system of unbounded Hermitian operators with dense domain in a reproducing kernel Hilbert space (RKHS) $\mathscr{H}_{F}$ associated to $F$.

Because of the role of positive definite functions in harmonic analysis, in statistics, and in physics, the connections in both directions is of interest, i.e., from (i) to (ii), and vice versa. This means that the notion of "extension" for question

(ii) must be inclusive enough in order to encompass all the extensions encountered in (i). For this reason enlargement of the initial Hilbert space $\mathscr{H}_{F}$ are needed. In other words, it is necessary to consider also operator extensions which are realized in a dilation-Hilbert space; a new Hilbert space containing $\mathscr{H}_{F}$ isometrically, and with the isometry intertwining the respective operators.

\subsection{Enlarging the Hilbert Space}

The purpose of this section is to describe this in detail, and to prove some lemmas which will then be used in chapter 3 , below. In chapter 3 , we identify extensions of the initial p.d. function $F$ which are associated with operator extensions in $\mathscr{H}_{F}$ (type 1), and those which require an enlargement of $\mathscr{H}_{F}$, type 2.

Let $F: \Omega-\Omega \rightarrow \mathbb{C}$ be a continuous p.d. function. Let $\mathscr{H}_{F}$ be the corresponding RKHS and $\xi_{x}:=F(x-\cdot) \in \mathscr{H}_{F}$.

$$
\left\langle\xi_{x}, \xi_{y}\right\rangle_{\mathscr{H}_{F}}=F(x-y), \forall x, y \in \Omega .
$$

As usual $F_{\varphi}=\varphi * F, \varphi \in C_{c}^{\infty}(\Omega)$. Then

$$
\begin{aligned}
\left\langle F_{\varphi}, F_{\psi}\right\rangle_{\mathscr{H}_{F}} & =\left\langle\xi_{0}, \pi\left(\varphi^{\#} * \psi\right) \xi_{0}\right\rangle \\
& =\left\langle\pi(\phi) \xi_{0}, \pi(\psi) \xi_{0}\right\rangle
\end{aligned}
$$

where $\pi(\varphi) \xi_{0}=F_{\varphi}$. The following lemma also holds in $\mathbb{R}^{n}$ with $n>1$, but we state it for $n=1$ to illustrate the "enlargement" of $\mathscr{H}_{F}$ question.

THEOREM 2.1.1. The following two conditions are equivalent:

(i) $F$ is extendable to a continuous p.d. function $\widetilde{F}$ defined on $\mathbb{R}$, i.e., $\widetilde{F}$ is a continuous p.d. function defined on $\mathbb{R}$ and $F(x)=\widetilde{F}(x)$ for all $x$ in $\Omega-\Omega$.

(ii) There is a Hilbert space $\mathscr{K}$, an isometry $W: \mathscr{H}_{F} \rightarrow \mathscr{K}$, and a strongly continuous unitary group $U_{t}: \mathscr{K} \rightarrow \mathscr{K}, t \in \mathbb{R}$ such that, if $A$ is the skew-adjoint generator of $U_{t}$, i.e.,

$$
\frac{1}{t}\left(U_{t} k-k\right) \rightarrow A k, \forall k \in \operatorname{dom}(A)
$$


then

and

$$
W F_{\varphi} \in \operatorname{domain}(A), \forall \varphi \in C_{c}^{\infty}(\Omega)
$$

$$
A W F_{\varphi}=W F_{\varphi^{\prime}}, \forall \varphi \in C_{c}^{\infty}(\Omega) .
$$

The rest of this section is devoted to the proof of Theorem 2.1.1.

Proof. $\mathbb{1}$ : First, assume there exists $\mathscr{K}, W, U_{t}$, and $A$ as in $(i i)$. Set

$$
\widetilde{F}(t)=\left\langle W \xi_{0}, U_{t} W \xi_{0}\right\rangle, t \in \mathbb{R} .
$$

Then, if $U_{t}=\int_{\mathbb{R}} e_{t}(\lambda) P(d \lambda)$, set

$$
d \mu(\lambda)=\left\|P(d \lambda) W \xi_{0}\right\|_{\mathscr{K}}^{2}=\left\langle W \xi_{0}, P(d \lambda) W \xi_{0}\right\rangle_{\mathscr{K}}
$$

and $\widetilde{F}=\widehat{d \mu}$ is the Bochner transform.

Lemma 2.1.2. If $s, t \in \Omega$, and $\varphi \in C_{c}^{\infty}(\Omega)$, then

$$
\left\langle W F_{\varphi}, U_{t} W F_{\varphi}\right\rangle=\left\langle\xi_{0}, \pi\left(\varphi^{\#} * \varphi_{t}\right) \xi_{0}\right\rangle_{\mathscr{H}_{F}},
$$

where $\varphi_{t}(\cdot)=\varphi(t-\cdot)$.

Suppose first (2.1.6) has been checked. Let $\phi_{\epsilon}$ be an approximate identity at $x=0$. Then

$$
\begin{aligned}
\widetilde{F}(t) & =\left\langle W \xi_{0}, U_{t} W \xi_{0}\right\rangle \\
& =\lim _{\epsilon \rightarrow 0}\left\langle\xi_{0}, \pi\left(\left(\phi_{\epsilon}\right)_{t}\right) \xi_{0}\right\rangle_{\mathscr{H}_{F}} \\
& =\left\langle\xi_{0}, \xi_{t}\right\rangle=F(t)
\end{aligned}
$$

by (2.1.5), (2.1.6), and (2.1.1).

Proof of Lemma 2.1.2. Now (2.1.6) follows from

$$
U_{t} W F_{\varphi}=W F_{\varphi_{t}},
$$

$\varphi \in C_{c}^{\infty}(\Omega), t \in \Omega$. Consider now

$$
U_{t-s} W F_{\varphi_{s}}= \begin{cases}U_{t} W F_{\varphi} & \text { at } s=0 \\ W F_{\varphi_{t}} & \text { at } s=t\end{cases}
$$

and

$$
\int_{0}^{t} \frac{d}{d s} U_{t-s} W F_{\varphi_{s}} d s=W F_{\varphi_{t}}-U_{t} W F_{\varphi} .
$$

We claim that the left hand side of (2.1.10) equals zero. By (2.1.2) and (2.1.3)

$$
\frac{d}{d s}\left[U_{t-s} W F_{\varphi_{s}}\right]=-U_{t-s} A W F_{\varphi_{s}}+U_{t-s} W F_{\varphi_{s}^{\prime}} .
$$

But, by (2.1.4) applied to $\varphi_{s}$, we get

$$
A W F_{\varphi_{s}}=W F_{\varphi_{s}^{\prime}}
$$

and the desired conclusion (2.1.8) follows.

$\underline{\Downarrow}$ : Assume $(i)$, let $\widetilde{F}=\widehat{d \mu}$ be a p.d. extension and Bochner transform. Then $\mathscr{H}_{\widetilde{F}} \simeq L^{2}(\mu) ;$ and for $\varphi \in C_{c}^{\infty}(\Omega)$, set

$$
W F_{\varphi}=\widetilde{F}_{\varphi}
$$

then $W: \mathscr{H}_{F} \rightarrow \mathscr{H}_{\widetilde{F}}$ is an isometry. 
Proof that (2.1.12) IS AN ISOMEtry . Let $\varphi \in C_{c}^{\infty}(\Omega)$. Then

$$
\begin{aligned}
\left\|\widetilde{F}_{\varphi}\right\|_{\mathscr{H}_{\widetilde{F}}^{2}}^{2} & =\iint \overline{\varphi(s)} \varphi(t) \widetilde{F}(t-s) d s d t \\
& =\left\|F_{\varphi}\right\|_{\mathscr{H}_{F}}^{2}=\int_{\mathbb{R}}|\widehat{\varphi}(\lambda)|^{2} d \mu(\lambda)
\end{aligned}
$$

since $\widetilde{F}$ is an extension of $F$.

Now set $U_{t}: L^{2}(\mu) \rightarrow L^{2}(\mu)$

$$
\left(U_{t} f\right)(\lambda)=e_{t}(\lambda) f(\lambda),
$$

a unitary group acting in $\mathscr{H}_{\widetilde{F}} \simeq L^{2}(\mu)$. Using (2.1.1), we get

$$
\left(W F_{\varphi}\right)(x)=\int e_{x}(\lambda) \widehat{\varphi}(\lambda) d \mu(\lambda), \forall x \in \Omega, \forall \varphi \in C_{c}^{\infty}(\Omega)
$$

And therefore (ii) follows. By (2.1.13)

$$
\begin{aligned}
\left(W F_{\varphi^{\prime}}\right)(x) & =\int e_{x}(\lambda) i \lambda \widehat{\varphi}(\lambda) d \mu(\lambda) \\
& =\left.\frac{d}{d t}\right|_{t=0} U_{t} W F_{\varphi} \\
& =A W F_{\varphi}
\end{aligned}
$$

as claimed.

An early instance of dilations (i.e., enlarging the Hilbert space) is the theorem by Sz.-Nagy [RSN56, Muh74] on unitary dilations of strongly continuous semigroups.

Theorem 2.1.3 (Sz.-Nagy). Let $\left\{S_{t}, t \in \mathbb{R}_{+}\right\}$be a strongly continuous semigroup of contractive operator in a Hilbert space $\mathscr{H}$; then there is

(1) a Hilbert space $\mathscr{K}$,

(2) an isometry $V: \mathscr{H} \rightarrow \mathscr{K}$,

(3) a strongly continuous one-parameter unitary group $\{U(t) \mid t \in \mathbb{R}\}$ acting on $\mathscr{K}$ such that

$$
V S_{t}=U(t) V, \forall t \in \mathbb{R}_{+}
$$

We mention this result here to stress that positive definite functions on $\mathbb{R}$ (and subsets of $\mathbb{R}$ ) often arise from contraction semigroups: Sz.-Nagy proved the following:

TheOREm 2.1.4 (Sz.-Nagy). Let $\left(S_{t}, \mathscr{H}\right)$ be a contraction semigroup, $t \geq 0$, (such that $S_{0}=I_{\mathscr{H}} ;$ ) and let $f_{0} \in \mathscr{H} \backslash\{0\}$; then the following function $F$ on $\mathbb{R}$ is positive definite:

$$
F(t)= \begin{cases}\left\langle f_{0}, S_{t} f_{0}\right\rangle_{\mathscr{H}} & \text { if } t \geq 0, \\ \left\langle f_{0}, S_{-t}^{*} f_{0}\right\rangle_{\mathscr{H}} & \text { if } t<0 .\end{cases}
$$

COROLlary 2.1.5. Every p.d. function as in (2.1.15) has the form:

$$
F(t):=\left\langle k_{0}, U(t) k_{0}\right\rangle_{\mathscr{K}}, t \in \mathbb{R}
$$

where $(U(t), \mathscr{K})$ is a unitary representation of $\mathbb{R}$. 


\section{2. $\operatorname{Ext}_{1}(F)$ and $\operatorname{Ext}_{2}(F)$}

Definition 2.2.1. Let $G$ be a locally compact group, and let $\Omega$ be an open connected subset of $G$. Let $F: \Omega^{-1} \cdot \Omega \rightarrow \mathbb{C}$ be a continuous positive definite function.

Consider a strongly continuous unitary representation $U$ of $G$ acting in some Hilbert space $\mathscr{K}$, containing the RKHS $\mathscr{H}_{F}$. We say that $(U, \mathscr{K}) \in \operatorname{Ext}(F)$ iff there is a vector $k_{0} \in \mathscr{K}$ such that

$$
F(g)=\left\langle k_{0}, U(g) k_{0}\right\rangle_{\mathscr{K}}, \forall g \in \Omega^{-1} \cdot \Omega
$$

I. The subset of $\operatorname{Ext}(F)$ consisting of $\left(U, \mathscr{H}_{F}, k_{0}=F_{e}\right)$ with

$$
F(g)=\left\langle F_{e}, U(g) F_{e}\right\rangle_{\mathscr{H}_{F}}, \forall g \in \Omega^{-1} \cdot \Omega
$$

is denoted $\operatorname{Ext}_{1}(F)$; and we set

$$
\operatorname{Ext}_{2}(F):=\operatorname{Ext}(F) \backslash \operatorname{Ext}_{1}(F) ;
$$

i.e., $\operatorname{Ext}_{2}(F)$ consists of the solutions to problem (2.2.1) for which $\mathscr{K} \supsetneqq \mathscr{H}_{F}$, i.e., unitary representations realized in an enlargement Hilbert space.

(We write $F_{e} \in \mathscr{H}_{F}$ for the vector satisfying $\left\langle F_{e}, \xi\right\rangle_{\mathscr{H}_{F}}=\xi(e), \forall \xi \in \mathscr{H}_{F}$, where $e$ is the neutral (unit) element in $G$, i.e., $e g=g, \forall g \in G$.)

II. In the special case, where $G=\mathbb{R}^{n}$, and $\Omega \subset \mathbb{R}^{n}$ is open and connected, we consider

$$
F: \Omega-\Omega \rightarrow \mathbb{C}
$$

continuous and positive definite. In this case,

$$
\begin{aligned}
\operatorname{Ext}(F)= & \left\{\mu \in \mathscr{M}_{+}\left(\mathbb{R}^{n}\right) \mid \widehat{\mu}(x)=\int_{\mathbb{R}^{n}} e^{i \lambda \cdot x} d \mu(\lambda)\right. \\
& \text { is a p.d. extensiont of } F\} .
\end{aligned}
$$

REMARK 2.2.2. Note that (2.2.3) is consistent with (2.2.1): For if $\left(U, \mathscr{K}, k_{0}\right)$ is a unitary representation of $G=\mathbb{R}^{n}$, such that (2.2.1) holds; then, by a theorem of Stone, there is a projection-valued measure (PVM) $P_{U}(\cdot)$, defined on the Borel subsets of $\mathbb{R}^{n}$ s.t.

$$
U(x)=\int_{\mathbb{R}^{n}} e^{i \lambda \cdot x} P_{U}(d \lambda), x \in \mathbb{R}^{n} .
$$

Setting

$$
d \mu(\lambda):=\left\|P_{U}(d \lambda) k_{0}\right\|_{\mathscr{K}}^{2},
$$

it is then immediate that we have: $\mu \in \mathscr{M}_{+}\left(\mathbb{R}^{n}\right)$, and that the finite measure $\mu$ satisfies

$$
\widehat{\mu}(x)=F(x), \forall x \in \Omega-\Omega .
$$

Set $n=1$ : Start with a local p.d. continuous function $F$, and let $\mathscr{H}_{F}$ be the corresponding RKHS. Let $\operatorname{Ext}(F)$ be the compact convex set of probability measures on $\mathbb{R}$ defining extensions of $F$.

Definition 2.2.3. For $\varphi \in C_{c}^{\infty}(\Omega)$, set

$$
\begin{aligned}
F_{\varphi}(x) & :=\int_{\Omega} \varphi(y) F(x-y) d y, \text { and } \\
D^{(F)}\left(F_{\varphi}\right) & :=F_{\frac{d \varphi}{d x}} ;
\end{aligned}
$$

then $D^{(F)}$ defines a skew-Hermitian operator with dense domain in $\mathscr{H}_{F}$. 
The role of deficiency indices (computed in $\mathscr{H}_{F}$ ) for the canonical skew-Hermitian operator in the RKHS $\mathscr{H}_{F}$ is as follows: the deficiency indices can be only $(0,0)$ or $(1,1)$.

We now divide $\operatorname{Ext}(F)$ into two parts, say $\operatorname{Ext}_{1}(F)$ and $\operatorname{Ext}_{2}(F)$.

Recall that all continuous p.d. extensions of $F$ come from strongly continuous unitary representations. So in the case of $1 \mathrm{D}$, from unitary one-parameter groups of course, say $U(t)$.

Further recall from section 2.1, that some of the p.d. extensions of $F$ may entail a bigger Hilbert space, say $\mathscr{K}$. By this we mean that $\mathscr{K}$ creates a dilation (enlargement) of $\mathscr{H}_{F}$ in the sense that $\mathscr{H}_{F}$ is isometrically embedded in $\mathscr{K}$. Via the embedding we may therefore view $\mathscr{H}_{F}$ as a closed subspace in $\mathscr{K}$.

Now let $\operatorname{Ext}_{1}(F)$ be the subset of $\operatorname{Ext}(F)$ corresponding to extensions when the unitary representation $U(t)$ acts in $\mathscr{H}_{F}$ (internal extensions), and $\operatorname{Ext}_{2}(F)$ denote the part of $\operatorname{Ext}(F)$ associated to unitary representations $U(t)$ acting in a proper enlargement Hilbert space $\mathscr{K}$ (if any), i.e., acting in a Hilbert space $\mathscr{K}$ corresponding to a proper dilation. The Polya extensions, see chapter 3 , account for a part of $\operatorname{Ext}_{2}(F)$. We have the following:

THEOREM 2.2.4. The deficiency indices computed in $\mathscr{H}_{F}$ are $(0,0)$ if and only if $\operatorname{Ext}_{1}(F)$ is a singleton.

REMARK 2.2.5. Even if $\operatorname{Ext}_{1}(F)$ is a singleton, we can still have non-empty $\operatorname{Ext}_{2}(F)$.

In chapter 3 , we include a host of examples, including one with a Polya extension where $\mathscr{K}$ is infinite dimensional, while $\mathscr{H}_{F}$ is 2 dimensional. (If $\mathscr{H}_{F}$ is 2 dimensional, then obviously we must have deficiency indices $(0,0)$.) In other examples we have $\mathscr{H}_{F}$ infinite dimensional, non-trivial Polya extensions and deficiency indices $(0,0)$.

Definition 2.2.6. Let $K_{i}, i=1,2$, be two positive definite kernels defined on some product $S \times S$ where $S$ is a set. We say that $K_{1} \ll K_{2}$ iff there is a finite constant $A$ such that

$$
\sum_{i} \sum_{j} \overline{c_{i}} c_{j} K_{1}\left(s_{i}, s_{j}\right) \leq A \sum_{i} \sum_{j} \overline{c_{i}} c_{j} K_{2}\left(s_{i}, s_{j}\right)
$$

for all finite systems $\left\{c_{i}\right\}$ of complex numbers.

If $F_{i}, i=1,2$, are positive definite functions defined on a subset of a group then we say that $F_{1} \ll F_{2}$ iff the two kernels

$$
K_{i}(x, y):=K_{F_{i}}(x, y)=F_{i}\left(x^{-1} y\right), i=1,2
$$

satisfies the condition in (2.2.9).

Lemma 2.2.7. Let $\mu_{i} \in \mathscr{M}_{+}\left(\mathbb{R}^{n}\right), i=1,2$, i.e., two finite positive Borel measures on $\mathbb{R}^{n}$, and let $F_{i}:=\widehat{d \mu_{i}}$ be the corresponding Bochner transforms. Then the following two conditions are equivalent:

(1) $\mu_{1} \ll \mu_{2}$ (meaning absolutely continuous) with $\frac{d \mu_{1}}{d \mu_{2}} \in L^{1}\left(\mu_{2}\right) \cap L^{\infty}\left(\mu_{2}\right)$.

(2) $F_{1} \ll F_{2}$, referring to the order of positive definite functions on $\mathbb{R}^{n}$.

PROOF. $\Downarrow$ If $(1)$ holds, then there is a Radon-Nikodym derivative $g \in L_{+}^{2}\left(\mathbb{R}^{n}, \mu_{2}\right)$ s.t.

$$
d \mu_{1}=g d \mu_{2}
$$


Let $\left\{c_{i}\right\}_{1}^{N},\left\{x_{i}\right\}_{1}^{N}$ be given: $c_{i} \in \mathbb{C}, x_{i} \in \mathbb{R}^{n} ;$ then

$$
\begin{aligned}
\sum_{j} \sum_{k} \overline{c_{j}} c_{k} F_{1}\left(x_{j}-x_{k}\right) & =\int_{\mathbb{R}^{n}}\left|\sum_{j} c_{j} e^{i x_{j} \lambda}\right|^{2} d \mu_{1}(\lambda) \\
& =\int_{\mathbb{R}^{n}}\left|\sum_{j} c_{j} e^{i x_{j} \lambda}\right|^{2} g(\lambda) d \mu_{2}(\lambda) \quad(\text { by } \quad(2.2 .10) \&(1)) \\
& \leq\|g\|_{L^{\infty}\left(\mu_{2}\right)} \int_{\mathbb{R}^{n}}\left|\sum_{j} c_{j} e^{i x_{j} \lambda}\right|^{2} d \mu_{2}(\lambda) \\
& =\|g\|_{L^{\infty}\left(\mu_{2}\right)} \sum_{j} \sum_{k} \overline{c_{j}} c_{k} F_{2}\left(x_{j}-x_{k}\right) .
\end{aligned}
$$

$\Uparrow$ If (2) holds, $\exists A<\infty$, s.t.

$$
\iint \overline{\varphi(x)} \varphi(y) F_{1}(x-y) d x d y \leq A \iint \overline{\varphi(x)} \varphi(y) F_{2}(x-y), \forall \varphi \in C_{c}(\mathbb{R}) .
$$

Using that $F_{i}=\widehat{d \mu_{i}}$, eq. $(2.2 .11)$ is equivalent to

$$
\int_{\mathbb{R}^{n}}|\widehat{\varphi}(\lambda)|^{2} d \mu_{1}(\lambda) \leq A \int_{\mathbb{R}^{n}}|\widehat{\varphi}(\lambda)|^{2} d \mu_{2}(\lambda)
$$

Now for the functions

$$
|\widehat{\varphi}(\lambda)|^{2}=\widehat{\varphi * \varphi^{\#}}(\lambda), \lambda \in \mathbb{R}^{n},
$$

we have that $\left\{\widehat{\psi}(\cdot) \mid \psi \in C_{c}\left(\mathbb{R}^{n}\right)\right\} \cap L^{1}\left(\mathbb{R}^{n}, \mu\right)$ is dense in $L^{1}\left(\mathbb{R}^{n}, \mu\right)$ for all $\mu \in$ $\mathscr{M}_{+}\left(\mathbb{R}^{n}\right)$.

It follows that $\mu_{1} \ll \mu_{2}$, and $g=\frac{d \mu_{1}}{d \mu_{2}} \in L_{+}^{1}\left(\mathbb{R}^{n}, \mu_{2}\right) \cap L^{\infty}\left(\mathbb{R}^{n}, \mu_{2}\right)$ by the argument in the first half of the proof.

\subsection{Preliminaries}

In the preliminary discussion below, we begin with the special case when $G=\mathbb{R}$, and when $\Omega$ is a bounded open interval.

Lemma 2.3.1. Fix $\Omega=(\alpha, \beta)$, let $a=\beta-\alpha$. Let $\alpha<x<\beta$ and let $\varphi_{n, x}(t)=$ $n \varphi(n(t-x))$, where $\varphi$ satisfies

(1) $\operatorname{supp}(\varphi) \subset(-a, a)$;

(2) $\varphi \in C_{c}^{\infty}, \varphi \geq 0$;

(3) $\int \varphi(t) d t=1$. Note that $\lim _{n \rightarrow \infty} \varphi_{n, x}=\delta_{x}$, the Dirac measure at $x$. Then

$$
\left\|F_{\varphi_{n, x}}-F(\cdot-x)\right\|_{\mathscr{H}_{F}} \rightarrow 0 \text {, as } n \rightarrow \infty \text {. }
$$

Hence $\left\{F_{\varphi}\right\}_{\varphi \in C_{c}^{\infty}(\Omega)}$ spans a dense subspace in $\mathscr{H}_{F}$.

Recall, the following facts about $\mathscr{H}_{F}$, which follow from the general theory [Aro50] of RKHS:

- $F(0)>0$, so we can always arrange $F(0)=1$.

- $F(-x)=\overline{F(x)}$

- $\mathscr{H}_{F}$ consists of continuous functions $\xi: \Omega-\Omega \rightarrow \mathbb{C}$. 


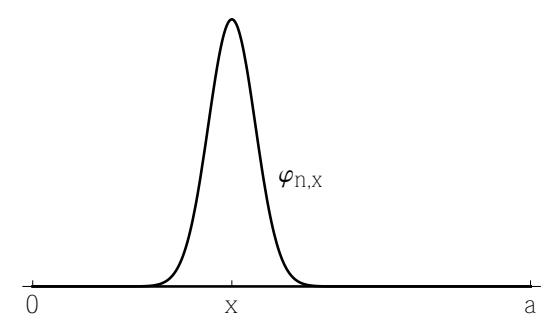

FIGURE 2.3.1. The approximate identity $\varphi_{n, x}(\cdot)$

- The reproducing property:

$$
\langle F(\cdot-x), \xi\rangle_{\mathscr{H}_{F}}=\xi(x), \forall \xi \in \mathscr{H}_{F}, \forall x \in \Omega,
$$

is a direct consequence of (1.5.4).

REMARK 2.3.2. It follows from the reproducing property that if $F_{\phi_{n}} \rightarrow \xi$ in $\mathscr{H}_{F}$, then $F_{\phi_{n}}$ converges uniformly to $\xi$ in $\Omega$. In fact

$$
\begin{aligned}
\left|F_{\phi_{n}}(x)-\xi(x)\right| & =\left|\left\langle F(\cdot-x), F_{\phi_{n}}-\xi\right\rangle_{\mathscr{H}_{F}}\right| \\
& \leq\|F(\cdot-x)\|_{\mathscr{H}_{F}}\left\|F_{\phi_{n}}-\xi\right\|_{\mathscr{H}_{F}} \\
& =F(0)\left\|F_{\phi_{n}}-\xi\right\|_{\mathscr{H}_{F}} .
\end{aligned}
$$

Theorem 2.3.3. A continuous function $\xi: \Omega \rightarrow \mathbb{C}$ is in $\mathscr{H}_{F}$ if and only if there exists $A_{0}>0$, such that

$$
\sum_{i} \sum_{j} \overline{c_{i}} c_{j} \overline{\xi\left(x_{i}\right)} \xi\left(x_{j}\right) \leq A_{0} \sum_{i} \sum_{j} \overline{c_{i}} c_{j} F\left(x_{i}-x_{j}\right)
$$

for all finite system $\left\{c_{i}\right\} \subset \mathbb{C}$ and $\left\{x_{i}\right\} \subset \Omega$.

Equivalently, for all $\psi \in C_{c}^{\infty}(\Omega)$,

$$
\left|\int_{\Omega} \psi(y) \xi(y) d y\right|^{2} \leq A_{0} \int_{\Omega} \int_{\Omega} \overline{\psi(x)} \psi(y) F(x-y) d x d y
$$

Note that, if $\xi \in \mathscr{H}_{F}$, then the LHS of (2.3.3) is $\left|\left\langle F_{\psi}, \xi\right\rangle_{\mathscr{H}_{F}}\right|^{2}$.

These two conditions $(2.3 .2)(\Leftrightarrow(2.3 .3))$ are the best way to characterize elements in the Hilbert space $\mathscr{H}_{F}$, where $F$ is a continuous positive definite function on $\Omega-\Omega$.

We will be using this when considering for example the deficiency-subspaces for skew-symmetric operators with dense domain in $\mathscr{H}_{F}$.

ExAmple 2.3.4. Let $G=\mathbb{T}=\mathbb{R} / \mathbb{Z}$, e.g., represented as $\left(-\frac{1}{2}, \frac{1}{2}\right]$. Fix $0<$ $a<\frac{1}{2}$, then $\Omega-\Omega=(-a, a) \bmod \mathbb{Z}$. So, for example, (1.5.1) takes the form $F:(-a, a) \bmod \mathbb{Z} \rightarrow \mathbb{C}$. See Figure 2.3.2. 

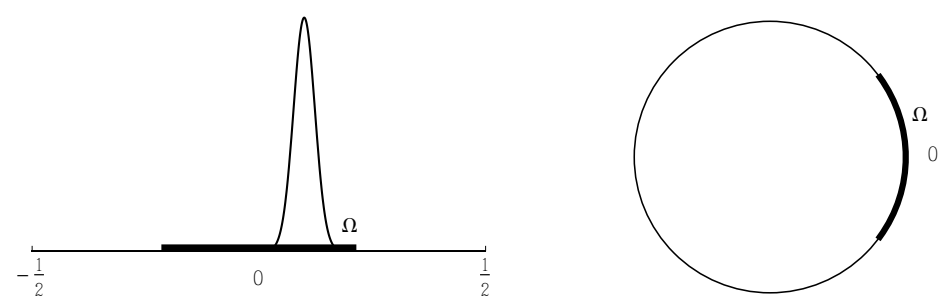

Figure 2.3.2. Two versions of $\Omega=(0, a) \subset \mathbb{T}^{1}$

2.3.1. The Operator $D^{(F)}$. Fix $0<a$ and a continuous positive definite function $F$ defined on $\Omega-\Omega$, where $\Omega=(0, a)$ as above. Let $\mathscr{H}_{F}$ be the corresponding RKHS as in (1.5.3).

Definition 2.3.5. Let $D^{(F)} F_{\psi}=F_{\psi^{\prime}}$, for all $\psi \in C_{c}^{\infty}(\Omega)$, where $\psi^{\prime}=\frac{d \psi}{d t}$ and $F_{\psi}$ is as in (1.5.5). In particular, the domain $\operatorname{dom}\left(D^{(F)}\right)$ of $D^{(F)}$ is the set of all $F_{\psi}, \psi \in C_{c}^{\infty}(\Omega)$.

Note that the recipe for $D^{(F)}$ yields a well-defined operator with dense domain in $\mathscr{H}_{F}$. To see this, use Schwarz' lemma to show that if $F_{\psi}=0$ in $\mathscr{H}_{F}$, then it follows that the vector $F_{\psi^{\prime}} \in \mathscr{H}_{F}$ is 0 as well.

Lemma 2.3.6. The operator $D^{(F)}$ is skew-symmetric and densely defined in $\mathscr{H}_{F}$.

Proof. By Lemma 1.5.3 dom $\left(D^{(F)}\right)$ is dense in $\mathscr{H}_{F}$. If $\psi \in C_{c}^{\infty}(0, a)$ and $|t|<\operatorname{dist}(\operatorname{supp}(\psi)$, endpoints), then

$$
\left\|F_{\psi(\cdot+t)}\right\|_{\mathscr{H}_{F}}^{2}=\left\|F_{\psi}\right\|_{\mathscr{H}_{F}}^{2}=\int_{0}^{a} \int_{0}^{a} \overline{\psi(x)} \psi(y) F(x-y) d x d y
$$

see (1.5.7), so

which is equivalent to

$$
\frac{d}{d t}\left\|F_{\psi(\cdot+t)}\right\|_{\mathscr{H}_{F}}^{2}=0
$$

$$
\left\langle D^{(F)} F_{\psi}, F_{\psi}\right\rangle_{\mathscr{H}_{F}}+\left\langle F_{\psi}, D^{(F)} F_{\psi}\right\rangle_{\mathscr{H}_{F}}=0 .
$$

It follows that $D^{(F)}$ is skew-symmetric.

To show that $D^{(F)} F_{\psi}=F_{\psi^{\prime}}$ is a well-defined operator on is dense domain in $\mathscr{H}_{F}$, we proceed as follows:

Lemma 2.3.7. The following implication holds:

$$
\begin{gathered}
{\left[\psi \in C_{c}^{\infty}(\Omega), F_{\psi}=0 \text { in } \mathscr{H}_{F}\right]} \\
\Downarrow \\
{\left[F_{\psi^{\prime}}=0 \text { in } \mathscr{H}_{F}\right]}
\end{gathered}
$$

Proof. Substituting (2.3.6) into

$$
\left\langle F_{\varphi}, F_{\psi^{\prime}}\right\rangle_{\mathscr{H}_{F}}+\left\langle F_{\varphi^{\prime}}, F_{\psi}\right\rangle_{\mathscr{H}_{F}}=0
$$


we get

$$
\left\langle F_{\varphi}, F_{\psi^{\prime}}\right\rangle_{\mathscr{H}_{F}}=0, \forall \varphi \in C_{c}^{\infty}(\Omega)
$$

Taking $\varphi=\psi^{\prime}$, yields

$$
\left\langle F_{\psi^{\prime}}, F_{\psi^{\prime}}\right\rangle=\left\|F_{\psi^{\prime}}\right\|_{\mathscr{H}_{F}}^{2}=0
$$

which is the desired conclusion (2.3.7).

This finishes the proof of Lemma 2.3.6.

Lemma 2.3.8. Let $\Omega=(\alpha, \beta)$. Suppose $F$ is a real valued positive definite function defined on $\Omega-\Omega$. The operator $J$ on $\mathscr{H}_{F}$ determined by

$$
J F_{\varphi}=\overline{F_{\varphi(\alpha+\beta-x)}}, \varphi \in C_{c}^{\infty}(\Omega)
$$

is a conjugation, i.e., $J$ is conjugate-linear, $J^{2}$ is the identity operator, and

$$
\left\langle J F_{\phi}, J F_{\psi}\right\rangle_{\mathscr{H}_{F}}=\left\langle F_{\psi}, F_{\phi}\right\rangle_{\mathscr{H}_{F}} .
$$

Moreover,

$$
D^{(F)} J=-J D^{(F)} .
$$

Proof. Let $a:=\alpha+\beta$ and $\phi \in C_{c}^{\infty}(\Omega)$. Since $F$ is real valued

$$
\begin{aligned}
J F_{\phi}(x) & =\int_{\alpha}^{\beta} \overline{\phi(a-y)} \overline{F(x-y)} d y \\
& =\int_{\alpha}^{\beta} \psi(y) F(x-y) d y
\end{aligned}
$$

where $\psi(y):=\overline{\phi(a-y)}$ is in $C_{c}^{\infty}(\Omega)$. It follows that $J$ maps the domain $\operatorname{dom}\left(D^{(F)}\right)$ of $D^{(F)}$ onto itself. For $\phi, \psi \in C_{c}^{\infty}(\Omega)$,

$$
\begin{aligned}
\left\langle J F_{\phi}, F_{\psi}\right\rangle_{\mathscr{H}_{F}} & =\int_{\alpha}^{\beta} F_{\phi(a-\cdot)}(x) \psi(x) d x \\
& =\int_{\alpha}^{\beta} \int_{\alpha}^{\beta} \phi(a-y) F(x-y) \psi(x) d y d x .
\end{aligned}
$$

Making the change of variables $(x, y) \rightarrow(a-x, a-y)$ and interchanging the order of integration we see that

$$
\begin{aligned}
\left\langle J F_{\phi}, F_{\psi}\right\rangle_{\mathscr{H}_{F}} & =\int_{\alpha}^{\beta} \int_{\alpha}^{\beta} \phi(y) F(y-x) \psi(a-x) d y d x \\
& =\int_{\alpha}^{\beta} \phi(y) F_{\psi(a-\cdot)}(y) d y \\
& =\left\langle J F_{\psi}, F_{\phi}\right\rangle_{\mathscr{H}_{F}},
\end{aligned}
$$

establishing (2.3.8). For $\phi \in C_{c}^{\infty}(\Omega)$,

$$
J D^{(F)} F_{\phi}=\overline{F_{\phi^{\prime}(a-\cdot)}}=-\overline{F_{\frac{d}{d x}(\phi(a-\cdot))}}=-D^{(F)} J F_{\phi},
$$

hence (2.3.9) holds. 
Definition 2.3.9. Let $\left(D^{(F)}\right)^{*}$ be the adjoint of $D^{(F)}$. The deficiency spaces $D E F^{ \pm}$consists of $\xi_{ \pm} \in \mathscr{H}_{F}$, such that $\left(D^{(F)}\right)^{*} \xi_{ \pm}= \pm \xi_{ \pm}$. That is,

$$
D E F^{ \pm}=\left\{\xi_{ \pm} \in \mathscr{H}_{F}:\left\langle F_{\psi^{\prime}}, \xi_{ \pm}\right\rangle_{\mathscr{H}_{F}}=\left\langle F_{\psi}, \pm \xi_{ \pm}\right\rangle_{\mathscr{H}_{F}}, \forall \psi \in C_{c}^{\infty}(\Omega)\right\}
$$

Corollary 2.3.10. If $F$ is real valued, then $D E F^{+}$and $D E F^{-}$have the same dimension.

Proof. This follows from Lemma 2.3.8, see e.g, [AG93] or [DS88].

Lemma 2.3.11. If $\xi \in D E F^{ \pm}$then $\xi(y)=$ constant $e^{\mp y}$.

Proof. Specifically, $\xi \in D E F^{+}$if and only if

$$
\int_{0}^{a} \psi^{\prime}(y) \xi(y) d y=\int_{0}^{a} \psi(y) \xi(y) d y, \forall \psi \in C_{c}^{\infty}(0, a) .
$$

Equivalently, $y \mapsto \xi(y)$ is a weak solution to

$$
-\xi^{\prime}=\xi
$$

i.e., a strong solution in $C^{1}$. Thus, $\xi(y)=$ constant $e^{-y}$. The $D E F^{-}$case is similar.

Corollary 2.3.12. Suppose $F$ is real valued. Let $\xi_{ \pm}(y):=e^{\mp y}$, for $y \in \Omega$. Then $\xi_{+} \in \mathscr{H}_{F}$ iff $\xi_{-} \in \mathscr{H}_{F}$. In the affirmative case $\left\|\xi_{-}\right\|_{\mathscr{H}_{F}}=e^{a}\left\|\xi_{+}\right\|_{\mathscr{H}_{F}}$.

Proof. Let $J$ be the conjugation from Lemma 2.3.8. A short calculation:

$$
\begin{aligned}
\left\langle J \xi, F_{\phi}\right\rangle_{\mathscr{H}_{\mathscr{F}}} & =\left\langle F_{\overline{\phi(a-\cdot)}}, \xi\right\rangle_{\mathscr{H}_{\mathscr{F}}}=\int \phi(a-x) \xi(x) d x \\
& =\int \phi(x) \xi(a-x) d x=\left\langle\overline{\xi(a-\cdot)}, F_{\phi}\right\rangle_{\mathscr{H}_{\mathscr{F}}}
\end{aligned}
$$

shows that $(J \xi)(x)=\overline{\xi(a-x)}$, for $\xi \in \mathscr{H}_{F}$. In particular, $J \xi_{-}=e^{a} \xi_{+}$. Since, $\left\|J \xi_{-}\right\|_{\mathscr{H}_{F}}=\left\|\xi_{-}\right\|_{\mathscr{H}_{F}}$, the proof is easily completed.

Corollary 2.3.13. The deficiency indices of $D^{(F)}$, with its dense domain in $\mathscr{H}_{F}$ are $(0,0),(0,1),(1,0)$, or $(1,1)$.

The second case in the above corollary happens precisely when $y \mapsto e^{-y} \in \mathscr{H}_{F}$. We can decide this with the use of $(2.3 .2)(\Leftrightarrow(2.3 .3))$.

In chapter 5 we will give some $a$ priori estimates, which enable us to strengthen Corollary 2.3.13 above. For this, see Corollary 5.4.19.

REMARK 2.3.14. Note that deficiency indices $(1,1)$ is equivalent to

$$
\begin{aligned}
\sum_{i} \sum_{j} \overline{c_{i}} c_{j} e^{-\left(x_{i}+x_{j}\right)} & \leq A_{0} \sum_{i} \sum_{j} \overline{c_{i}} c_{j} F\left(x_{i}-x_{j}\right) \\
& \mathbb{1} \\
\left|\int_{0}^{a} \psi(y) e^{-y} d y\right|^{2} & \leq A_{0} \int_{0}^{a} \int_{0}^{a} \overline{\psi(x)} \psi(y) F(x-y) d x d y
\end{aligned}
$$

But it depends on $F$ (given on $(-a, a)$ ).

Lemma 2.3.15. On $\mathbb{R} \times \mathbb{R}$, define the following kernel $K_{+}(x, y)=e^{-|x+y|}$, $(x, y) \in \mathbb{R} \times \mathbb{R}$; then this is a positive definite kernel on $\mathbb{R}_{+} \times \mathbb{R}_{+}$; (see [Aro50] for details on positive definite kernels.) 

Then

Proof. Let $\left\{c_{j}\right\} \subset \mathbb{C}^{N}$ be a finite system of numbers, and let $\left\{x_{j}\right\} \subset \mathbb{R}_{+}^{N}$.

$$
\sum_{j} \sum_{k} \overline{c_{j}} c_{k} e^{-\left(x_{j}+x_{k}\right)}=\left|\sum_{j} c_{j} e^{-x_{j}}\right|^{2} \geq 0
$$

Corollary 2.3.16. Let $F, \mathscr{H}_{F}$, and $D^{(F)}$ be as in Corollary 2.3.13; then $D^{(F)}$ has deficiency indices $(1,1)$ if and only if the kernel $K_{+}(x, y)=e^{-|x+y|}$ is dominated by $K_{F}(x, y):=F(x-y)$ on $(0, a) \times(0, a)$, i.e., there is a finite positive constant $A_{0}$ such that $A_{0} K_{F}-K_{+}$is positive definite on $(0, a) \times(0, a)$.

Proof. This is immediate from the lemma and (2.3.10) above.

By Corollary 2.3.13, we conclude that there exists skew-adjoint extension $A^{(F)} \supset$ $D^{(F)}$ in $\mathscr{H}_{F}$. That is, $\left(A^{(F)}\right)^{*}=-A^{(F)}$, and $\left\{F_{\psi}\right\}_{\psi \in C_{c}^{\infty}(0, a)} \subset \operatorname{dom}\left(A^{(F)}\right) \subset \mathscr{H}_{F}$.

Hence, set $U(t)=e^{t A^{(F)}}: \mathscr{H}_{F} \rightarrow \mathscr{H}_{F}$, and get the unitary one-parameter group

$$
\{U(t): t \in \mathbb{R}\}, U(s+t)=U(s) U(t), \forall s, t \in \mathbb{R}
$$

and if

$$
\xi \in \operatorname{dom}\left(A^{(F)}\right)=\left\{\xi \in \mathscr{H}_{F}: \text { s.t. } \lim _{t \rightarrow 0} \frac{U(t) \xi-\xi}{t} \text { exists }\right\}
$$

then

$$
A^{(F)} \xi=\lim _{t \rightarrow 0} \frac{U(t) \xi-\xi}{t} .
$$

Now use $F_{x}(\cdot)=F(x-\cdot)$ defined in $(0, a)$; and set

$$
F_{A}(t):=\left\langle F_{0}, U(t) F_{0}\right\rangle_{\mathscr{H}_{F}}, \forall t \in \mathbb{R}
$$

then using (2.3.1), we see that $F_{A}$ is a continuous positive definite extension of $F$ on $(-a, a)$, i.e., a continuous positive definite function on $\mathbb{R}$, and if $x \in(0, a)$, then we get the following conclusion:

LEMMA 2.3.17. $F_{A}$ is a continuous bounded positive definite function of $\mathbb{R}$ and

$$
F_{A}(t)=F(t) .
$$

for $t \in(-a, a)$.

Proof. But $\mathbb{R} \ni t \mapsto F_{A}(t)$ is bounded and continuous, since $\{U(t)\}$ is a strongly continuous unitary group acting on $\mathscr{H}_{F}$, and

$$
\left|F_{A}(t)\right|=\left|\left\langle F_{0}, U(t) F_{0}\right\rangle\right| \leq\left\|F_{0}\right\|_{\mathscr{H}_{F}}\left\|U(t) F_{0}\right\|_{\mathscr{H}_{F}}=\left\|F_{0}\right\|_{\mathscr{H}_{F}}^{2}
$$

where $\left|\left\langle F_{0}, U(t) F_{0}\right\rangle\right| \leq\left\|F_{0}\right\|_{\mathscr{H}_{F}}^{2}=F(0)$, see (2.10.4). See the proof of Theorem 2.1.1 and [Jor89, Jor90, Jor91b] for the remaining details.

REMARK 2.3.18. In the circle case $\mathbb{T}$ the function $F_{A}$ in $(2.3 .12)$ needs not be $\mathbb{Z}$-periodic, and we want a positive definite continuous function on $\mathbb{T}$; hence in this case we are not yet done.

$F$ can be normalized by $F(0)=1$. Recall that $F$ is defined on $(-a, a)=\Omega-\Omega$ if $\Omega=(0, a)$. 
Consider the spectral representation:

$$
U(t)=\int_{-\infty}^{\infty} e_{t}(\lambda) P(d \lambda)
$$

where $e_{t}(\lambda)=e^{i 2 \pi \lambda t}$; and $P(\cdot)$ is a projection-valued measure on $\mathbb{R}, P(B): \mathscr{H}_{F} \rightarrow$ $\mathscr{H}_{F}, \forall B \in \operatorname{Borel}(\mathbb{R})$. Then

$$
d \mu(\lambda)=\left\|P(d \lambda) F_{0}\right\|_{\mathscr{H}_{F}}^{2}
$$

satisfies

$$
F_{A}(t)=\int_{-\infty}^{\infty} e_{t}(\lambda) d \mu(\lambda), \forall t \in \mathbb{R}
$$

Conclusion. The extension $F_{A}$ from (2.3.12) has nice transform properties, and via (2.3.15) we get

where $\mathscr{H}_{F_{A}}$ is the RKHS of $F_{A}$.

$$
\mathscr{H}_{F_{A}} \simeq L^{2}(\mathbb{R}, \mu)
$$

\subsection{The Case of Locally Compact Abelian Groups}

We are concerned with extensions of locally defined continuous and positive definite (p.d.) functions $F$ on Lie groups, say $G$, but some results apply to locally compact groups as well. However in the case of locally compact Abelian groups, we have stronger theorems, due to the powerful Fourier analysis theory for locally compact Abelian groups.

We must fix notations:

- $G$ : a given locally compact abelian group, write the operation in $G$ additively;

- $d x$ : denotes the Haar measure of $G$ (unique up to a scalar multiple.)

- $\widehat{G}$ : the dual group, i.e., $\widehat{G}$ consists of all continuous homomorphisms: $\lambda: G \rightarrow \mathbb{T}, \lambda(x+y)=\lambda(x) \lambda(y), \forall x, y \in G ; \lambda(-x)=\overline{\lambda(x)}, \forall x \in G$. Occasionally, we shall write $\langle\lambda, x\rangle$ for $\lambda(x)$. Note that $\widehat{G}$ also has its Haar measure.

THEOREM 2.4.1 (Pontryagin [Rud90]). $\widehat{\widehat{G}} \simeq G$, and we have the following:

$$
[G \text { is compact }] \Longleftrightarrow[\widehat{G} \text { is discrete }]
$$

Let $\phi \neq \Omega \subset G$ be an open connected subset, and let $F: \Omega-\Omega \rightarrow \mathbb{C}$ be a fixed continuous positive definite (p.d.) function. We choose the normalization $F(0)=1$.

Given $F$, we now introduce the corresponding reproducing kernel Hilbert space, (RKHS) for short:

Lemma 2.4.2. For $\varphi \in C_{c}(\Omega)$, set

$$
F_{\varphi}(\cdot)=\int_{\Omega} \varphi(y) F(\cdot-y) d y
$$

then $\mathscr{H}_{F}$ is the Hilbert completion of $\left\{F_{\varphi} \mid \varphi \in C_{c}(\Omega)\right\}$ in the inner product:

$$
\left\langle F_{\varphi}, F_{\psi}\right\rangle_{\mathscr{H}_{F}}=\int_{\Omega} \int_{\Omega} \overline{\varphi(x)} \psi(y) F(x-y) d x d y .
$$

Here $C_{c}(\Omega):=$ all continuous compactly supported functions in $\Omega$. 
Lemma 2.4.3. The Hilbert space $\mathscr{H}_{F}$ is also a Hilbert space of continuous functions on $\Omega$ as follows:

If $\xi: \Omega \rightarrow \mathbb{C}$ is a fixed continuous function, then $\xi \in \mathscr{H}_{F}$ if and only if $\exists$ $K=K_{\xi}<\infty$ such that

$$
\left|\int_{\Omega} \overline{\xi(x)} \varphi(x) d x\right|^{2} \leq K \int_{\Omega} \int_{\Omega} \overline{\varphi\left(y_{1}\right)} \varphi\left(y_{2}\right) F\left(y_{1}-y_{2}\right) d y_{1} d y_{2} .
$$

When (2.4.3) holds, then

$$
\left\langle\xi, F_{\varphi}\right\rangle_{\mathscr{H}_{F}}=\int_{\Omega} \overline{\xi(x)} \varphi(x) d x, \text { for all } \varphi \in C_{c}(\Omega) .
$$

Proof. We refer to the basics on the theory of RKHSs; e.g., [Aro50].

Lemma 2.4.4. There is a bijective correspondence between all continuous p.d. extensions $\tilde{F}$ to $G$ of the given p.d. function $F$ on $\Omega-\Omega$, on the one hand; and all Borel probability measures $\mu$ on $\widehat{G}$, on the other, i.e., all $\mu \in \mathscr{M}(\widehat{G})$ s.t.

$$
F(x)=\widehat{\mu}(x), \forall x \in \Omega-\Omega
$$

where

$$
\widehat{\mu}(x)=\int_{\widehat{G}} \lambda(x) d \mu(\lambda)=\int_{\widehat{G}}\langle\lambda, x\rangle d \mu(\lambda), \forall x \in G .
$$

Proof. This is an immediate application of Bochner's characterization of the continuous positive definite functions on locally compact abelian groups.

Definition 2.4.5. Set

$$
\operatorname{Ext}(F)=\{\mu \in \mathscr{M}(\widehat{G}) \mid \text { s.t. (2.4.4) holds }\} .
$$

Remark 2.4.6. There are examples where $\operatorname{Ext}(F)=\phi$. See section 2.7 where $G=\mathbb{T}$

Lemma 2.4.7. Ext $(F)$ is weak $*$-compact and convex.

Proof. Left to the reader; see e.g., [Rud73].

THEOREM 2.4.8.

(1) Let $F$ and $\mathscr{H}_{F}$ be as above; and let $\mu \in \mathscr{M}(\widehat{G})$; then there is a positive Borel function $h$ on $\widehat{G}$ s.t. $h^{-1} \in L^{\infty}(\widehat{G})$, and $h d \mu \in \operatorname{Ext}(F)$, if and only if $\exists K_{\mu}<\infty$ such that

$$
\int_{\widehat{G}}|\widehat{\varphi}(\lambda)|^{2} d \mu(\lambda) \leq K_{\mu} \int_{\Omega} \int_{\Omega} \overline{\varphi\left(y_{1}\right)} \varphi\left(y_{2}\right) F\left(y_{1}-y_{2}\right) d y_{1} d y_{2} .
$$

(2) Assume $\mu \in \operatorname{Ext}(F)$, then

$$
(f d \mu)^{\vee} \in \mathscr{H}_{F}, \forall f \in L^{2}(\widehat{G}, \mu) .
$$

Proof. The assertion in (2.4.5) is immediate from Lemma 2.4.3.

Our conventions for the two transforms used in (2.4.5) and (2.4.6) are as follows:

$$
\widehat{\varphi}(\lambda)=\int_{G} \overline{\langle\lambda, x\rangle} \varphi(x) d x
$$

if $\varphi \in C_{c}(\Omega)$, of course $\widehat{\varphi}(\lambda)=\int_{G} \overline{\langle\lambda, x\rangle} \varphi(x) d x$. 
The transform in (2.4.6) is:

$$
(f d \mu)^{\vee}=\int_{\widehat{G}}\langle\lambda, x\rangle f(\lambda) d \mu(\lambda) .
$$

The notation $(f d \mu)^{\vee}$ may be more logical.

The remaining computations are left to the reader.

Corollary 2.4.9.

(1) Let $F$ be as above; then $\mu \in \operatorname{Ext}(F)$ iff the following operator

$$
T\left(F_{\varphi}\right)=\widehat{\varphi}, \varphi \in C_{c}(\Omega)
$$

is well-defined on $\mathscr{H}_{F}$, and bounded as follows: $T: \mathscr{H}_{F} \rightarrow L^{2}(\widehat{G}, \mu)$.

(2) In this case, the adjoint operator $T^{*}: L^{2}(\widehat{G}, \mu) \rightarrow \mathscr{H}_{F}$ is given by

$$
T^{*}(f)=(f d \mu)^{\vee}, \forall f \in L^{2}(\widehat{G}, \mu) .
$$

Proof. If $\mu \in \operatorname{Ext}(F)$, then for all $\varphi \in C_{c}(\Omega)$, and $x \in \Omega$, we have (see $(2.4 .1))$

$$
\begin{aligned}
F_{\varphi}(x) & =\int_{\Omega} \varphi(y) F(x-y) d y \\
& =\int_{\Omega} \varphi(y) \widehat{\mu}(x-y) d y \\
& =\int_{\Omega} \varphi(y)\langle\lambda, x-y\rangle d \mu(\lambda) d y \\
& \stackrel{\text { Fubini }}{=} \int_{\widehat{G}}\langle\lambda, x\rangle \widehat{\varphi}(\lambda) d \mu(\lambda) .
\end{aligned}
$$

By Lemma 2.4.3, we note that $(\widehat{\varphi} d \mu)^{\vee} \in \mathscr{H}_{F}$, see (2.4.8). Hence $\exists K<\infty$ such that the estimate (2.4.5) holds. To see that $T\left(F_{\varphi}\right)=\widehat{\varphi}$ is well-defined on $\mathscr{H}_{F}$, we must check the implication:

$$
\left(F_{\varphi}=0 \text { in } \mathscr{H}_{F}\right) \Longrightarrow\left(\widehat{\varphi}=0 \text { in } L^{2}(\widehat{G}, \mu)\right)
$$

but this now follows from estimate (2.4.5).

Using the definition of the respective inner products in $\mathscr{H}_{F}$ and in $L^{2}(\widehat{G}, \mu)$, we check directly that, if $\varphi \in C_{c}(\Omega)$, and $f \in L^{2}(\widehat{G}, \mu)$ then we have:

$$
\langle\widehat{\varphi}, f\rangle_{L^{2}(\mu)}=\left\langle F_{\varphi},(f d \mu)^{\vee}\right\rangle_{\mathscr{H}_{F}} .
$$

On the RHS in (2.4.10), we note that, when $\mu \in \operatorname{Ext}(F)$, then $\widehat{f d \mu} \in \mathscr{H}_{F}$. This last conclusion is a consequence of Lemma 2.4.3. Indeed, since $\mu$ is finite, $L^{2}(\widehat{G}, \mu) \subset L^{1}(\widehat{G}, \mu)$, so $\widehat{f d \mu}$ in $(2.4 .8)$ is continuous on $G$ by Riemann-Lebesgue; and so is its restriction to $\Omega$. If $\mu$ is further assumed absolutely continuous, then $\widehat{f d \mu} \rightarrow 0$ at $\infty$.

With a direct calculation, using the reproducing property in $\mathscr{H}_{F}$, and Fubini's theorem, we check directly that the following estimate holds:

$$
\left|\int_{\Omega} \overline{\varphi(x)}(f d \mu)^{\vee}(x) d x\right|^{2} \leq\left(\int_{\Omega} \int_{\Omega} \overline{\varphi\left(y_{1}\right)} \varphi\left(y_{2}\right) F\left(y_{1}-y_{2}\right) d y_{1} d y_{2}\right)\|f\|_{L^{2}(\mu)}^{2}
$$

and so Lemma 2.4.3 applies; we get $(f d \mu)^{\vee} \in \mathscr{H}_{F}$. 
It remains to verify the formula (2.4.10) for all $\varphi \in C_{c}(\Omega)$ and all $f \in L^{2}(\widehat{G}, \mu)$; but this now follows from the reproducing property in $\mathscr{H}_{F}$, and Fubini.

Once we have this, both assertions in (1) and (2) in the Corollary follow directly from the definition of the adjoint operator $T^{*}$ with respect to the two Hilbert spaces in $\mathscr{H}_{F} \stackrel{T}{\longrightarrow} L^{2}(\widehat{G}, \mu)$. Indeed then (2.4.9) follows.

We recall a general result on continuity of positive definite (p.d.) functions on any locally compact Lie group:

THEOREM 2.4.10. If $F$ is p.d. function on a locally compact group $G$, assumed continuous only in a neighborhood of $e \in G$; then it is automatically continuous everywhere on $G$.

Proof. Since $F$ is positive definite, we may apply the Gelfand-Naimark-Segal (GNS) theorem to get a cyclic unitary representation $(U, \mathscr{H}, v), v$ denoting the cyclic vector, such that $F(g)=\langle v, U(g) v\rangle, g \in G$. The stated assertion about continuity for unitary representations is easy to verify; and so it follows for $F$.

Question. Suppose $\operatorname{Ext}(F) \neq \phi$, then what are its extreme points? Equivalently, characterize $\operatorname{ext}(\operatorname{Ext}(F))$.

Let $\Omega \subset G, \Omega \neq \phi, \Omega$ open and connected, and let " $"$ denote the Fourier transform in the given locally compact Abelian group $G$. Set

$$
K_{\Omega}(\lambda)=\widehat{\chi_{\Omega}(\lambda)}, \forall \lambda \in \widehat{G},
$$

where $\widehat{G}$ is the dual character group (see Theorem 2.4.1).

TheOrem 2.4.11. Let $F: \Omega-\Omega \rightarrow \mathbb{C}$ be continuous, and positive definite on $\Omega-\Omega$; and assume $\operatorname{Ext}(F) \neq \phi$. Let $\mu \in \operatorname{Ext}(F)$, and let $T_{\mu}\left(F_{\phi}\right):=\widehat{\varphi}$, defined initially only for $\varphi \in C_{c}(\Omega)$, be the isometry $T_{\mu}: \mathscr{H}_{F} \rightarrow L^{2}(\mu)=L^{2}(\widehat{G}, \mu)$. Then $Q_{\mu}:=T_{\mu} T_{\mu}^{*}$ is a projection in $L^{2}(\mu)$ with $K_{\Omega}(\cdot)$ as kernel:

$$
\left(Q_{\mu} f\right)(\lambda)=\int_{\widehat{G}} K_{\Omega}(\lambda-\xi) f(\xi) d \mu(\xi), \forall f \in L^{2}(\widehat{G}, \mu), \forall \lambda \in \widehat{G} .
$$

Proof. We showed in Theorem 2.4.8 that $T_{\mu}: \mathscr{H}_{F} \rightarrow L^{2}(\mu)$ is isometric, and so $Q_{\mu}:=T_{\mu} T_{\mu}^{*}$ is the projection in $L^{2}(\mu)$. For $f \in L^{2}(\mu), \lambda \in \widehat{G}$, we have the following computation, where the interchanging of integrals is justified by Fubini's theorem:

$$
\begin{aligned}
\left(Q_{\mu} f\right)(\lambda) & =\int_{\Omega}(f d \mu)^{\vee}(x)\langle\lambda, x\rangle d x(\text { where } d x \text { is Haar measure on G) } \\
& =\int_{\Omega}\langle\lambda, x\rangle\left(\int_{\widehat{G}} f(\xi) \overline{\langle\xi, x\rangle} d \mu(\xi)\right) d x \\
& \stackrel{\text { Fubini }}{=} \int_{\widehat{G}} K_{\Omega}(\lambda-\xi) f(\xi) d \mu(\xi)
\end{aligned}
$$

which is the desired conclusion (2.4.11).

\subsection{The Case of $G=\mathbb{R}^{n}$}

Of course the case of $G=\mathbb{R}^{n}$ is a special case of the setting of locally compact Abelian groups from above, and the results available for $\mathbb{R}^{n}$ are more refined. We focus on this in the present section. This is also the setting of the more classical studies of extension questions. 
Setting. Let $\Omega \subset \mathbb{R}^{n}$ be a fixed open and connected subset; and let $F: \Omega-\Omega \rightarrow$ $\mathbb{C}$ be a given continuous and positive definite function defined on

$$
\Omega-\Omega:=\left\{x-y \in \mathbb{R}^{n} \mid x, y \in \Omega\right\} .
$$

Let $\mathscr{H}_{F}$ be the corresponding reproducing kernel Hilbert space (RKHS). We showed that $\operatorname{Ext}(F) \neq \phi$ if and only if there is a strongly continuous unitary representation $\{U(t)\}_{t \in \mathbb{R}^{n}}$ acting on $\mathscr{H}_{F}$ such that

$$
\mathbb{R}^{n} \ni t \mapsto\left\langle F_{0}, U(t) F_{0}\right\rangle_{\mathscr{H}_{F}}
$$

is a p.d. extension of $F$, extending from (2.5.1) to $\mathbb{R}^{n}$. Finally, if $U$ is a unitary representation of $G=\mathbb{R}^{n}$ we denote by $P_{U}(\cdot)$ the associated projection valued measure $(\mathrm{PVM})$ on $\mathscr{B}\left(\mathbb{R}^{n}\right)$ (= the sigma-algebra of all Borel subsets in $\left.\mathbb{R}^{n}\right)$.

We have

$$
U(t)=\int_{\mathbb{R}^{n}} e^{i t \cdot \lambda} P_{U}(d \lambda), \forall t \in \mathbb{R}^{n}
$$

where $t=\left(t_{1}, \ldots, t_{n}\right), \lambda=\left(\lambda_{1}, \ldots, \lambda_{n}\right)$, and $t \cdot \lambda=\sum_{j=1}^{n} t_{j} \lambda_{j}$. Recall, setting

$$
d \mu(\cdot)=\left\|P_{U}(\cdot) F_{0}\right\|_{\mathscr{H}_{F}}^{2},
$$

then the p.d. function on RHS in (2.5.2) satisfies

$$
\operatorname{RHS}_{(2.5 .2)}=\int_{\mathbb{R}^{n}} e^{i t \cdot \lambda} d \mu(\lambda), \forall t \in \mathbb{R}^{n} .
$$

The purpose of the next theorem is to give an orthogonal splitting of the RKHS $\mathscr{H}_{F}$ associated to a fixed $(\Omega, F)$ when it is assumed that $\operatorname{Ext}(F)$ is non-empty. This orthogonal splitting of $\mathscr{H}_{F}$ depends on a choice of $\mu \in \operatorname{Ext}(F)$, and the splitting is into three orthogonal subspaces of $\mathscr{H}_{F}$, correspond a splitting of spectral types into atomic, completely continuous (with respect to Lebesgue measure), and singular.

THEOREM 2.5.1. Let $\Omega \subset \mathbb{R}^{n}$ be given, $\Omega \neq \phi$, open and connected. Suppose $F$ is given p.d. and continuous on $\Omega-\Omega$, and assume $\operatorname{Ext}(F) \neq \phi$. Let $U$ be the corresponding unitary representations of $G=\mathbb{R}^{n}$, and let $P_{U}(\cdot)$ be its associated PVM acting on $\mathscr{H}_{F}$ (= the RKHS of F.)

(1) Then $\mathscr{H}_{F}$ splits up as an orthogonal sum of three closed and $U(\cdot)$ invariant subspaces

$$
\mathscr{H}_{F}=\mathscr{H}_{F}^{(\text {atom })} \oplus \mathscr{H}_{F}^{(\text {ac })} \oplus \mathscr{H}_{F}^{(\text {sing })}
$$

with these subspaces characterized as follows:

The PVM $P_{U}(\cdot)$ restricted to $\mathscr{H}_{F}^{(\text {atom })},\left(\mathscr{H}_{F}^{(a c)}\right.$, resp., $\left.\mathscr{H}_{F}^{(\text {sing })}\right)$ is purely atomic, is absolutely continuous with respect to Lebesgue measure $d \lambda=$ $d \lambda_{1} \cdots d \lambda_{n}$ on $\mathbb{R}^{n}$, respectively, $P_{U}(\cdot)$ is continuous, purely singular, when restricted to $\mathscr{H}_{F}^{(\text {sing) }}$.

(2) Case $\mathscr{H}_{F}^{(\text {atom })}$. If $\lambda \in \mathbb{R}^{n}$ is an atom in $P_{U}(\cdot)$, i.e., $P_{U}(\{\lambda\}) \neq 0$, where $\{\lambda\}$ denotes the singleton with $\lambda$ fixed; then $P_{U}(\{\lambda\}) \mathscr{H}_{F}$ is onedimensional, and the function $e_{\lambda}(x):=e^{i \lambda \cdot x}$, (complex exponential) restricted to $\Omega$, is in $\mathscr{H}_{F}$. We have:

$$
P_{U}(\{\lambda\}) \mathscr{H}_{F}=\left.\mathbb{C} e_{\lambda}\right|_{\Omega} .
$$


Case $\mathscr{H}_{F}^{(a c)}$. If $\xi \in \mathscr{H}_{F}^{(a c)}$, then it is represented as a continuous function on $\Omega$, and

$\left\langle\xi, F_{\varphi}\right\rangle_{\mathscr{H}_{F}}=\int_{\Omega} \overline{\xi(x)} \varphi(x) d x($ Lebesgue meas. $), \forall \varphi \in C_{c}(\Omega)$.

Moreover, there is a $f \in L^{2}\left(\mathbb{R}^{n}, \mu\right)$ (where $\mu$ is given in (2.5.4) such that

$$
\int_{\Omega} \overline{\xi(x)} \varphi(x) d x=\int_{\mathbb{R}^{n}} \overline{f(\lambda)} \widehat{\varphi}(\lambda) d \mu(\lambda), \forall \varphi \in C_{c}(\Omega) ;
$$

and

$$
\xi=\left.(f d \mu)^{\vee}\right|_{\Omega} .
$$

(We say that $(f d \mu)^{\vee}$ is the $\mu$-extension of $\xi$.)

Conclusion. Every $\mu$-extension of $\xi$ is continuos on $\mathbb{R}^{n}$, and goes to 0

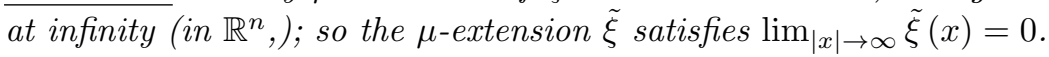

Case $\mathscr{H}_{F}^{(\text {sing })}$. Vectors $\xi \in \mathscr{H}_{F}^{(\text {sing })}$ are characterized by the following property:

The measure

$$
d \mu_{\xi}(\cdot):=\left\|P_{U}(\cdot) \xi\right\|_{\mathscr{H}_{F}}^{2}
$$

is continuous and purely singular.

Proof. Most of the proof details are contained in the previous discussion.

For (2), Case $\mathscr{H}_{F}^{(\text {atom) }}$; suppose $\lambda \in\left(\mathbb{R}^{n}\right)$ is an atom, and that $\xi \in \mathscr{H}_{F} \backslash\{0\}$ satisfies

$$
P_{U}(\{\lambda\}) \xi=\xi
$$

then

$$
U(t) \xi=e^{i t \cdot \lambda} \xi, \forall t \in \mathbb{R}^{n}
$$

Using now (2.5.2)-(2.5.3), we conclude that $\xi$ (as a continuous function on $\mathbb{R}^{n}$ ) is a weak solution to the following elliptic system

$$
\frac{\partial}{\partial x_{j}} \xi=\sqrt{-1} \lambda_{j} \xi(\text { on } \Omega), 1 \leq j \leq n .
$$

Hence $\xi=$ const $\left.\cdot e_{\lambda}\right|_{\Omega}$ as asserted in (2).

Case (2), $\mathscr{H}_{F}^{(a c)}$ follows from (2.5.10) and the Riemann-Lebesgue theorem applied to $\mathbb{R}^{n}$; and case $\mathscr{H}_{F}^{(s i n g)}$ is immediate.

EXAmPle 2.5.2. Consider the following continuous positive definite function $F$ on $\mathbb{R}$, or on some bounded interval $(-a, a), a>0$.

$$
F(x)=\frac{1}{3}\left(e^{-i x}+\prod_{n=1}^{\infty} \cos \left(\frac{2 \pi x}{3^{n}}\right)+e^{i 3 x / 2} \frac{\sin (x / 2)}{(x / 2)}\right) .
$$

(1) This is the decomposition (2.5.6) of the corresponding RKHSs $\mathscr{H}_{F}$, all three subspaces $\mathscr{H}_{F}^{(\text {atom })}, \mathscr{H}_{F}^{(a c)}$, and $\mathscr{H}_{F}^{(\text {sing) }}$ are non-zero; the first one is one-dimensional, and the other two are infinite-dimensional. 


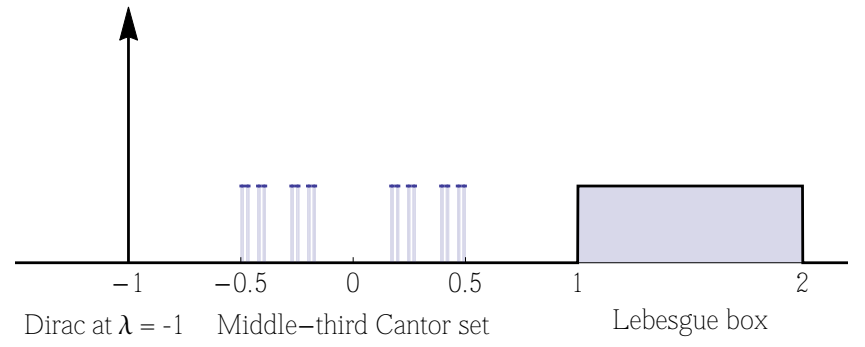

Figure 2.5.1. The measure $d \mu(\lambda)$ in example 2.5.2.

(2) The operator

$$
\begin{gathered}
D^{(F)}\left(F_{\varphi}\right):=F_{\varphi^{\prime}} \text { on } \\
\operatorname{dom}\left(D^{(F)}\right)=\left\{F_{\varphi} \mid \varphi \in C_{c}^{\infty}(0, a)\right\}
\end{gathered}
$$

is bounded, and so extends by closure to a skew-adjoint operator $\overline{D^{(F)}}=$ $-\left(D^{(F)}\right)^{*}$.

PROOF. Using infinite convolutions of operators (see chapter 6), and results from [DJ12], we conclude that $F$ defined in $(2.5 .15)$ is entire analytic, and $F=\widehat{d \mu}$ (Bochner-transform) where

$$
d \mu(\lambda)=\frac{1}{3}\left(\delta_{-1}+\mu_{\text {Cantor }}+\chi_{[1,2]}(\lambda) d \lambda\right) .
$$

The measures on the RHS in (2.5.17) are as follows:

- $\delta_{-1}$ is the Dirac mass at -1 , i.e., $\delta(\lambda+1)$.

- $\mu_{\text {Cantor }}=$ the middle-third Cantor measure $\mu_{c}$ determined as the unique solution in $\mathscr{M}_{+}^{\text {prob }}(\mathbb{R})$ to

$$
\int f(\lambda) d \mu_{c}(\lambda)=\frac{1}{2}\left(\int f\left(\frac{\lambda+1}{3}\right) d \mu_{c}(\lambda)+\int f\left(\frac{\lambda-1}{3}\right) d \mu_{c}\right)
$$

for all $f \in C_{c}(\mathbb{R})$; and the last term

- $\chi_{[1,2]}(\lambda) d \lambda$ is restriction to the closed interval $[1,2]$ of Lebesgue measure.

See Figure 2.5.1-2.5.2.

It follows from the literature (e.g. [DJ12]) that $\mu_{c}$ is supported in $\left[-\frac{1}{2}, \frac{1}{2}\right]$; and so the three measures on the RHS in (2.5.17) have disjoint compact support, with the three supports positively separately.

The conclusions asserted in the example follow from this, in particular the properties for $D^{(F)}$, in fact

$$
\operatorname{spectrum}\left(D^{(F)}\right) \subseteq\{-1\} \cup\left[-\frac{1}{2}, \frac{1}{2}\right] \cup[1,2]
$$

COROLlaRY 2.5.3. Let $\Omega \subset \mathbb{R}^{n}$ be non-empty, open and connected subset. Let $F: \Omega-\Omega \rightarrow \mathbb{C}$ be a fixed continuous and positive definite function; and let $\mathscr{H}_{F}$ be the corresponding RKHS (of functions on $\Omega$.) 


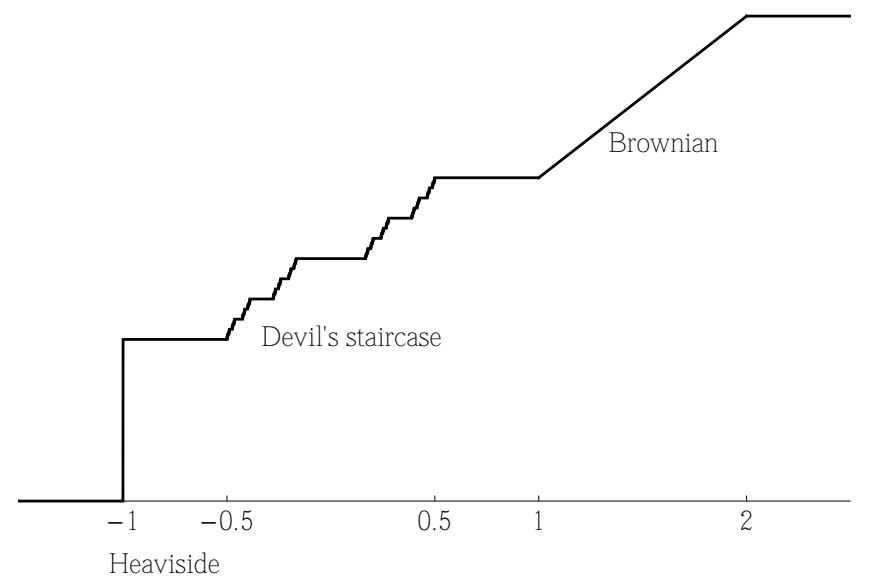

Figure 2.5.2. Cumulative distribution $=\int_{-\infty}^{\lambda} d \mu(\lambda)$, as in example 2.5.2.

(1) If there is a compactly supported measure $\mu \in \operatorname{Ext}(F)$, then every function $\xi$ on $\Omega$, which is in $\mathscr{H}_{F}$, has an entire analytic extension to $\mathbb{C}^{n}$, i.e., extension from $\Omega \subset \mathbb{R}^{n}$ to $\mathbb{C}^{n}$.

(2) If, in addition, it is assume that $\mu \ll d \lambda\left(=d \lambda_{1} \cdots d \lambda_{n}\right)=$ the Lebesgue measure on $\mathbb{R}^{n}$, where "«" means "absolutely continuous;" then the constant non-zero functions on $\Omega$ are NOT in $\mathscr{H}_{F}$.

Proof. Part (1). Let $\Omega, F, \mathscr{H}_{F}$ and $\mu$ be as in the statement of the corollary. Let

$$
T\left(F_{\varphi}\right):=\widehat{\varphi}, \varphi \in C_{c}(\Omega)
$$

be the isometry $\mathscr{H}_{F} \stackrel{T}{\longrightarrow} L^{2}\left(\mathbb{R}^{n}, \mu\right)$ from Corollary 2.4.9. By Coroll. 2.4.9 (2); for all $f \in L^{2}\left(\mathbb{R}^{n}, \mu\right)$, we have

$$
\left(T^{*} f\right)(x)=\int_{\mathbb{R}^{n}} e^{i x \cdot \lambda} f(\lambda) d \mu(\lambda) ; x \in \Omega ;
$$

and further that

$$
T^{*}\left(L^{2}\left(\mathbb{R}^{n}, \mu\right)\right)=\mathscr{H}_{F} .
$$

Now, if $\mu$ is of compact support, then so is the complex measure $f d \mu$. This measure is finite since $L^{2}(\mu) \subset L^{1}(\mu)$. Hence the desired conclusion follows from (2.5.20), (2.5.21), and the Paley-Wiener theorem; see e.g., [Rud73].

We now prove the assertion in part (2) of the corollary. But this follows from Theorem 2.5.1, case $\mathscr{H}_{F}^{(c c)}$. See also Proposition 2.10.8 below.

\subsection{Lie Groups}

Definition 2.6.1. Let $G$ be a Lie group. We consider the extension problem for continuous positive definite functions

$$
F: \Omega^{-1} \Omega \rightarrow \mathbb{C}
$$


where $\Omega \neq \phi$, is a connected and open subset in $G$, i.e., it is assumed that

$$
\sum_{i} \sum_{j} \overline{c_{i}} c_{j} F\left(x_{i}^{-1} x_{j}\right) \geq 0
$$

for all finite systems $\left\{c_{i}\right\} \subset \mathbb{C}$, and points $\left\{x_{i}\right\} \subset \Omega$.

Equivalent,

$$
\int_{\Omega} \overline{\varphi(x)} \varphi(y) F\left(x^{-1} y\right) d x d y \geq 0
$$

for all $\varphi \in C_{c}(\Omega)$; where $d x$ denotes a choice of left-invariant Haar measure on $G$.

Lemma 2.6.2. Let $F$ be defined as in (2.6.1)-(2.6.2); and for all $X \in L a(G)=$ the Lie algebra of $G$, set

$$
(\tilde{X} \varphi)(g):=\frac{d}{d t} \varphi\left(\exp _{G}(-t X) g\right)
$$

for all $\varphi \in C_{c}^{\infty}(\Omega)$. Set

$$
F_{\varphi}(x):=\int_{\Omega} \varphi(y) F\left(x^{-1} y\right) d y ;
$$

then

$$
S_{X}^{(F)}\left(F_{\varphi}\right):=F_{\tilde{X} \varphi}, \varphi \in C_{c}^{\infty}(\Omega)
$$

defines a representation of the Lie algebra $L a(G)$ by skew-Hermitian operators in the RKHS $\mathscr{H}_{F}$, with the operator in (2.6.6) defined on the common dense domain $\left\{F_{\varphi} \mid \varphi \in C_{c}^{\infty}(\Omega)\right\} \subset \mathscr{H}_{F}$.

Proof. The arguments here follow those of the proof of lemma 2.3.6 mutatis mutandis.

DEFinition 2.6.3.

(1) We say that a continuous p.d. function $F: \Omega^{-1} \Omega \rightarrow \mathbb{C}$ is extendable iff there is a continuous p.d. function $F_{e x}: G \rightarrow G$ such that

$$
\left.F_{e x}\right|_{\Omega^{-1} \Omega}=F .
$$

(2) Let $U \in \operatorname{Rep}(G, \mathscr{K})$ be a strongly continuous unitary representation of $G$ acting in some Hilbert space $\mathscr{K}$. We say that $U \in \operatorname{Ext}(F)$ iff (Def.) there is an isometry $\mathscr{H}_{F} \hookrightarrow \mathscr{K}$ such that the function

$$
G \ni g \mapsto\left\langle J F_{e}, U(g) J F_{e}\right\rangle_{\mathscr{K}}
$$

satisfies the condition in (1).

THEOREM 2.6.4. Every extension of some continuous p.d. function $F$ on $\Omega^{-1} \Omega$ as in (1) arises from a unitary representation of $G$ as specified in (2).

Proof. First assume some unitary representation $U$ of $G$ satisfies (2), then (2.6.8) is an extension of $F$. This follows from the argument in our proof of lemma 2.3.6.

For the converse; assume some continuous p.d. function $F_{e x}$ on $G$ satisfies (2.6.7). Now apply the GNS-theorem to $F_{e x}$; and, as a result, we get a cyclic representation $\left(U, \mathscr{K}, v_{0}\right)$ where

- $\mathscr{K}$ is a Hilbert space;

- $U$ is a strongly continuous unitary representation of $G$ acting on $\mathscr{K}$; and 
- $v_{0} \in \mathscr{K}$ is a cyclic vector, $\left\|v_{0}\right\|=1$; and

$$
F_{\text {ex }}(g)=\left\langle v_{0}, U(g) v_{0}\right\rangle, g \in G .
$$

Defining now $J: \mathscr{H}_{F} \rightarrow \mathscr{K}$ as follows,

$$
J(F(\cdot g)):=U\left(g^{-1}\right) v_{0}, \forall g \in \Omega ;
$$

and extension by limit, we check that $J$ is isometric and satisfies the condition from (2) in Definition 2.6.3. We omit details as they parallel arguments already contained in chapter 1.

Theorem 2.6.5. Let $\Omega, G, L a(G)$, and $F: \Omega^{-1} \Omega \rightarrow \mathbb{C}$ be as in Definition 2.6.1. Let $\tilde{G}$ be the simply connected universal covering group for $G$. Then $F$ has an extension to a p.d. continuous function on $\tilde{G}$ iff there is a unitary representation $U$ of $\tilde{G}$ and an isometry $\mathscr{H}_{F} \stackrel{J}{\longrightarrow} \mathscr{K}$ such that

$$
J S_{X}^{(F)}=d U(X) J
$$

holds on $\left\{F_{\varphi} \mid \varphi \in C_{c}^{\infty}(\Omega)\right\}$, for all $X \in L a(G)$; where

$$
d U(X) U(\varphi) v_{0}=U(\tilde{X} \varphi) v_{0} .
$$

Proof. Details are contained in sections 2.1, 2.7, and chapter 3.

Assume $G$ is connected. Note that on $C_{c}^{\infty}(\Omega)$, the Lie group $G$ acts locally, i.e., by $\varphi \mapsto \varphi_{g}$ where $\varphi_{g}$ denotes translation of $\varphi$ by some element $g \in G$, such that $\varphi_{g}$ is also supported in $\Omega$. Then

$$
\left\|F_{\varphi}\right\|_{\mathscr{H}_{F}}=\left\|F_{\varphi_{g}}\right\|_{\mathscr{H}_{F}}
$$

but only for elements $g \in G$ in a neighborhood of $e \in G$, and with the neighborhood depending on $\varphi$.

Corollary 2.6.6. Given

$$
F: \Omega^{-1} \cdot \Omega \rightarrow \mathbb{C}
$$

continuous and positive definite, then set

$$
L_{g}\left(F_{\varphi}\right):=F_{\varphi_{g}}, \varphi \in C_{c}^{\infty}(\Omega),
$$

defining a local representation of $G$ in $\mathscr{H}_{E}$, see [Jor87, Jor86].

Corollary 2.6.7. Given F, positive definite and continuous, as in (2.6.12), and let $L$ be the corresponding local representation of $G$ acting on $\mathscr{H}_{F}$. Then $\operatorname{Ext}(F) \neq \phi$ if and only if the local representation (2.6.13) extends to a global unitary representation acting in some Hilbert space $\mathscr{K}$, containing $\mathscr{H}_{F}$ isometrically.

Proof. We refer to [Jor87, Jor86] for details, as well as chapter 4 below. 


\subsection{The Circle Group $\mathbb{T}$}

While we consider extensions of locally defined continuous and positive definite (p.d.) functions $F$ on groups, say $G$, the question of whether $G$ is simply connected or not plays an essential role in the analysis, and in the possible extensions. It turns out that the geometric issues arising for general Lie groups can be illustrated well in a simple case: To illustrate this point, we isolate below the two groups, the circle group, and its universal cover, the real line. We study extensions defined on a small arc in the circle group $G=\mathbb{T}=\mathbb{R} / \mathbb{Z}$, versus extensions to the universal covering group $\mathbb{R}$.

Let $G=\mathbb{T}=\mathbb{R} / \mathbb{Z}$ represented as $\left(-\frac{1}{2}, \frac{1}{2}\right]$. Pick $0<\varepsilon<\frac{1}{2}$, set $\Omega=(0, \varepsilon)$, then $\Omega-\Omega=(-\varepsilon, \varepsilon) \bmod \mathbb{Z}$.

LEMMA 2.7.1. If $L$ has deficiency indices $(1,1)$, there is a selfadjoint extension $A$ of $L$ acting in $\mathscr{H}_{F}$, such that the corresponding p.d. extension $\widetilde{F}$ of $F$ has period one, then $\varepsilon$ is rational.

Proof. In the notation of Theorem 2.1.1 our assumptions imply that $\mathscr{K}=$ $\mathscr{H}_{F}$. Suppose the deficiency indices of $D^{(F)}=L$ are $(1,1)$, the generator of $U_{A}(t)$, $A$ is a selfadjoint extension of $D^{(F)}$, and

$$
F_{A}(t)=\left\langle F_{0}, U_{A}(t) F_{0}\right\rangle_{F}=\int_{\mathbb{R}} e_{t}(\lambda) d \mu(\lambda)
$$

has period one, where

$$
U_{A}(t)=\int_{\mathbb{R}} e_{t}(\lambda) P_{A}(d \lambda)
$$

and

$$
d \mu_{A}(\lambda)=\left\|P_{A}(d \lambda) F_{0}\right\|_{F}^{2} .
$$

Since the deficiency indices are $(1,1)$, the selfadjoint extensions of $D^{(F)}$ are determined by boundary conditions of the form

$$
\xi(\varepsilon)=e(\theta) \xi(0),
$$

where $0 \leq \theta<1$ is fixed. Let $A_{\theta}$ be the s.a. corresponding to $\theta$. Repeating the calculation of the defect spaces and using that eigenfunctions must satisfy the boundary condition, it follows that the spectrum of $A_{\theta}$ the

$$
\lambda_{n}=\frac{\theta+n}{\varepsilon},
$$

$n \in \mathbb{Z}$ for which $e_{\lambda_{n}}$ is in $\mathscr{H}_{F}$.

The support of $\mu_{\theta}$ is a subset of the spectrum of $A_{\theta}$. (The support is equal to the spectrum, if $F$ is a cyclic vector for $U_{t}$.) Since $F_{A}$ is has period one, the support of $\mu_{\theta}$ to consist of integers. If $\lambda_{n}$ and $\lambda_{m}$ are in the support of $\mu_{\theta}$, then

$$
\varepsilon=\frac{n-m}{\lambda_{n}-\lambda_{m}} .
$$

It follows that $\varepsilon$ is rational. 


\subsection{Example: $e^{i 2 \pi x}$}

This is a trivial example, but it is helpful to understand why the extensions of positive definite functions is a quite different question from the other extensions we looked at before.

Here we refer to classes of unbounded Hermitian operators which arise in scattering theory for wave equations in physics; see e.g., [PT13, JPT12a, LP89, LP85]. However the context of these questions is different. For a discussion of connections, see e.g., section 7.2.1, and Lemma 7.2.5 below.

Take our momentum operator on $L^{2}\left(-\frac{1}{2}, \frac{1}{2}\right)$, where $\left(-\frac{1}{2}, \frac{1}{2}\right] \simeq \mathbb{R} / \mathbb{Z} \simeq \mathbb{T}$.

One of the extensions is characterized by

(1) $(U(t) f)(x)=f(x+t \bmod 1), f \in L^{2}(\mathbb{R} / \mathbb{Z})$; and if $\xi_{n}(x):=e^{i 2 \pi n x}$, then $U(t) \xi_{n}=e_{n}(t) \xi_{n}$.

(2) Take

$$
F(\cdot):=\left.e_{1}(\cdot)\right|_{(-\epsilon, \epsilon)}
$$

where $0<\epsilon<\frac{1}{2}$.

LEMma 2.8.1. The RKHS $\mathscr{H}_{F}$ of $F$ in (2.8.1) is one-dimensional.

Proof. Recall that $\mathscr{H}_{F}$ is the completion of

$$
\left\{F_{\varphi}: \varphi \in C_{c}^{\infty}(0, \epsilon)\right\}
$$

with

$$
\begin{aligned}
F_{\varphi}(x) & =\int_{0}^{\epsilon} \varphi(y) F(x-y) d y \\
& =e_{1}(x) \int_{0}^{\epsilon} \varphi(y) \overline{e_{1}(y)} d y \\
& =e_{1}(x) \widehat{\varphi}(1)
\end{aligned}
$$

and note that $\operatorname{span}\left\{F_{\varphi}: \varphi \in C_{c}^{\infty}(0, \epsilon)\right\}$ is one-dimensional.

Recall that

$$
\begin{aligned}
& \left\langle F_{\varphi}, F_{\psi}\right\rangle_{\mathscr{H}_{F}}=\int_{0}^{\epsilon} \int_{0}^{\epsilon} \overline{\varphi(x)} \psi(y) F(y-x) d x d y \\
& =\overline{\widehat{\varphi}(1)} \widehat{\psi}(1), \forall \varphi, \psi \in C_{c}^{\infty}(0, \epsilon) \text {; }
\end{aligned}
$$

and

$$
\left\|F_{\varphi}\right\|_{\mathscr{H}_{F}}^{2}=|\widehat{\varphi}(1)|^{2} \text {. }
$$

Now this $F$ is defined as a restriction (of $e_{1}(\cdot)$ ) and so it has at least this extension $e_{1}(\cdot)$. It is also the unique continuous positive definite extension to $\mathbb{T}$.

Proof of the uniqueness assertion: Case $\mathbb{T}$. If $F$ is a continuous p.d. extension of $e_{1}$ to $\mathbb{T}$, then by Bochner's theorem,

$$
e_{1}(x)=\sum_{n \in \mathbb{Z}} e_{n}(x) \mu_{n}, \forall x \in(-\epsilon, \epsilon)
$$

where $\mu_{n} \geq 0$, and $\sum_{n \in \mathbb{Z}} \mu_{n}=1$. Now each side of the above equation extends continuously in $x$. By uniqueness of the Fourier expansion in (2.8.2), we get $\mu_{1}=1$, and $\mu_{n}=0, n \in \mathbb{Z} \backslash\{1\}$. 
We argue as follows: By change of summation index in (2.8.2), we get

$$
1 \equiv \sum_{n \in \mathbb{Z}} e_{n}(x) \mu_{n+1},|x|<\epsilon .
$$

Writing $e_{n}(x)=\cos (2 \pi n x)+i \sin (2 \pi n x)$, we note that (2.8.2) is equivalent to a cosine, and a sine expansion. By a change of variable, we are left with proving the following implication:

$$
\left[\sum_{n=1}^{\infty} k_{n} \cos (2 \pi n x) \equiv 0, k_{n} \geq 0,|x|<\epsilon\right] \Longrightarrow\left[k_{1}=k_{2}=\cdots=0\right] ;
$$

and a similar implication for the sine expansion. Now for all $N \in \mathbb{N}$, we then write the infinite sum in (2.8.3) as

$$
\underbrace{\sum_{n>N} k_{n} \cos (2 \pi n x)}_{A_{N}}+\underbrace{\sum_{n=1}^{N} k_{n} \cos (2 \pi n x)}_{B_{N}} \equiv 0 \text { in }|x|<\epsilon .
$$

But if $|x|<\frac{\epsilon}{4 N}$, then $\cos (2 \pi n x)>0,1 \leq n \leq N$. Since $A_{N} \rightarrow 0$ uniformly, we conclude that $k_{1}=k_{2}=\cdots=0$.

\subsection{Example: $e^{-a|x|}$}

Consider $\Omega=(0, \epsilon) \subset \mathbb{R}$ v.s. $\Omega=(0, \epsilon) \subset \mathbb{T}$. In both cases, we assume $F$ is positive definite and continuous on $(-\epsilon, \epsilon)=\Omega-\Omega$, but there are many more solutions $\tilde{F}$, continuous, positive definite on $\mathbb{R}$ than there are periodic solutions $\tilde{F}_{\text {per }}$ on $\mathbb{T}=\mathbb{R} / \mathbb{Z}$.

FACT 2.9.1. A solution $\tilde{F}$ to the $\mathbb{R}$-problem is

$$
\tilde{F}(t)=\left\langle F_{0}, U(t) F_{0}\right\rangle_{\mathscr{H}_{F}}, t \in \mathbb{R} .
$$

The $\mathbb{T}$-problem is equivalent to

$$
\tilde{F}_{\text {per }}(t+n)=\tilde{F}_{\text {per }}(t), \forall t \in \mathbb{R}, \forall n \in \mathbb{Z} .
$$

Note that

$$
\tilde{F}_{\text {per }}(t)=\sum_{k \in \mathbb{Z}} e^{i 2 \pi k t} w_{k}, w_{k} \geq 0, \sum_{k} w_{k}<\infty
$$

i.e., a Fourier transform of a finite measure on $\mathbb{Z}$,

$$
\mu_{\mathbb{Z}}(A)=\sum_{k \in \mathbb{Z} \cap A} w_{k}, \sum_{k \in \mathbb{Z}} w_{k}<\infty .
$$

EXAMPlE 2.9.2. Fix $a>0, w_{k}=\frac{1}{a^{2}+k^{2}}$, then

$$
\tilde{F}_{p e r}(t)=\frac{1}{\pi} \sum_{k \in \mathbb{Z}} \frac{e^{i 2 \pi k t}}{a^{2}+k^{2}}=\sum_{n \in \mathbb{Z}} e^{-a|t-n|}
$$

by the Poisson summation formula. This is a function on $\mathbb{T}=\mathbb{R} / \mathbb{Z}$, and the RHS of $(2.9 .3)$ is a positive definite continuous on $\mathbb{R} / \mathbb{Z}$ and an extension of its restriction to $(0, \epsilon) \subset \mathbb{T}$. 

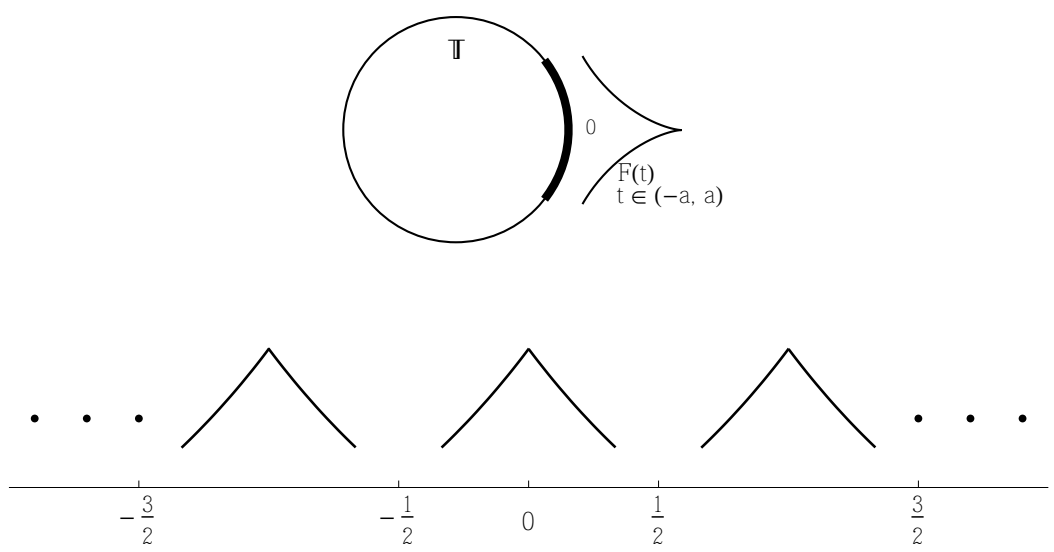

Note this bijection also applies if a continuous p.d. function $F$ is only defined on a subset $(-a, a) \subset \mathbb{T}$, with $0<a<\frac{1}{2}$.

FiguRE 2.9.1. Functions on $\mathbb{T}=\mathbb{R} / \mathbb{Z} \longleftrightarrow$ (1-periodic functions on $\mathbb{R})$.

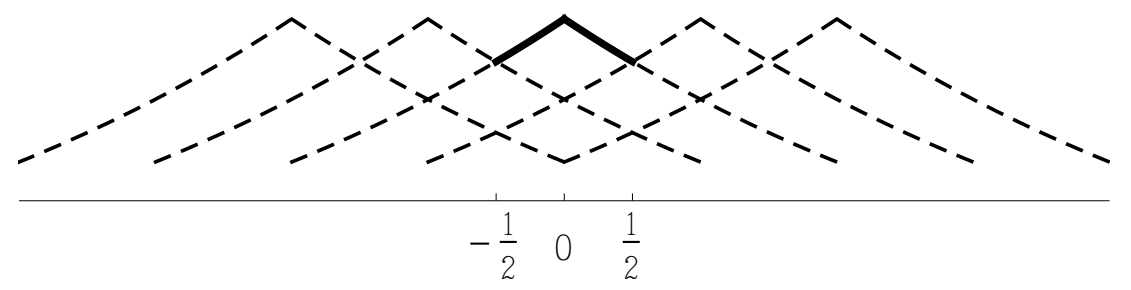

Figure 2.9.2. $\tilde{F}_{\text {per }}(t)>F(t)$ on $\left[-\frac{1}{2}, \frac{1}{2}\right]$

REMARK 2.9.3. There is a bijection between

(i) functions on $\mathbb{T}=\mathbb{R} / \mathbb{Z}$, and

(ii) 1-periodic functions on $\mathbb{R}$.

Note that (ii) include positive definite functions $F$ defined only on a subset $(-\epsilon, \epsilon) \subset \mathbb{T}$. Hence, it is tricky to get extension $\tilde{F}$ to $\mathbb{T}=\mathbb{R} / \mathbb{Z}$.

Example 2.9.4. Fix $a>0$. Let $F(t)=e^{-a|t|}$, for all $t \in(-\epsilon, \epsilon)$. See Figure 2.9.1. Then $\tilde{F}(t)=e^{-a|t|}, t \in \mathbb{R}$, is a continuous positive definite extension to $\mathbb{R}$. The periodic version

$$
\tilde{F}_{p e r}(t)=\sum_{n \in \mathbb{Z}} e^{-a|t-n|}
$$

is continuous, positive definite on $\mathbb{T}$; but $\tilde{F}_{\text {per }}(t)$ is not an extension of $F$. See Figure 2.9.2. Note $\tilde{F}_{\text {per }}(t)>\tilde{F}(t)=e^{-a|t|}$ on $\mathbb{R}$, since

$$
\tilde{F}_{p e r}(t)=\underbrace{e^{-a|t|}}_{F(t)}+\underbrace{\sum_{n \in \mathbb{Z} \backslash\{0\}} e^{-a|t-n|}}_{>0}
$$


REMARK 2.9.5. If $0<\epsilon<\frac{1}{2}$ is given, and if $F$ is continuous and positive definite on $(-\epsilon, \epsilon) \subset \mathbb{T}=\mathbb{R} / \mathbb{Z}$, then the analysis of $\mathscr{H}_{F}$ is totally independent of considerations of periods. $\mathscr{H}_{F}$ does not see global properties. To understand $F$ on $(-\epsilon, \epsilon)$ we only need one period interval; see Fig (ii), but when we pass to $\mathbb{R}$, things can be complicated. See Fig (ii)-(iii).

Question. In the example $F(t)=e^{-a|t|}, t \in(-\epsilon, \epsilon)$, what are the deficiency indices of $D^{(F)}$, i.e., the densely defined skew-Hermitian operator on $\mathscr{H}_{F}, F_{\psi} \mapsto$ $F_{\psi^{\prime}}, \psi \in C_{c}^{\infty}(0, \epsilon)$, understood as an operator in $\mathscr{H}_{F}$ ?

It may be $(0,0)$. We must decide if the two functions $\xi_{ \pm}(t)=e^{ \pm t},|t|<\epsilon$, are in the RKHS $\mathscr{H}_{F}$. To do this we use the estimates $(2.3 .2)(\Leftrightarrow(2.3 .3))$ above. Or we can solve with Laplace transform.

But we do know the RHS of (2.9.3) is a periodization of a positive definite function on $\mathbb{R}$, i.e.,

$$
t \mapsto \frac{1}{\pi} e^{-a|t|}=\int_{-\infty}^{\infty} e^{i 2 \pi \lambda t} \frac{d \lambda}{a^{2}+\lambda^{2}}
$$

and hence (2.9.3) is an application of the Poisson-summation formula.

\subsection{The Example $e^{-|x|}$ in $(-a, a)$}

While section 2.5 deals with the case of $G=\mathbb{R}^{n}$, here we set $n=1$. With this restriction we are able to answer classical questions about positive definite functions, their harmonic analysis, and their operator theory. As an application of this we obtain a spectral classification of all Hermitian operators with dense domain in a separable Hilbert space, having deficiency indices $(1,1)$. Further spectral theoretic results are given in chapter 8 .

Here we study the following example $F(x)=e^{-|x|}$ on any fixed and finite interval, $|x|<a$. So $a$ is fixed; it can be any positive real number, and we study the case when the group $G$ is $\mathbb{R}$.

When we compute the associated RKHS $\mathscr{H}_{F}$ from this $F$, and its skew Hermitian operator in $\mathscr{H}_{F}$, we get deficiency indices $(1,1)$. This is a special example, but still enlightening in connection with deficiency index considerations. But, for contrast, we note that, in general, there will be a host of other interesting $1 \mathrm{D}$ examples of partially defined continuous positive definite functions. Some will have indices $(1,1)$ and others $(0,0)$.

In case $(1,1)$, the convex set $\operatorname{Ext}(F)$ is parameterized by $\mathbb{T}$; while, in case $(0,0), \operatorname{Ext}(F)$ is a singleton.

Setting: Fix $a, 0<a<\infty$. Set $\Omega=(0, a)$, so $\Omega-\Omega=(-a, a)$; and let

$$
F(x):=e^{-|x|}, \text { for }|x|<a .
$$

Note, we do not define $F$ outside $(-a, a)$.

We shall need the following probability measure $\mu$ on $\mathbb{R}$ :

$$
d \mu(\lambda)=\frac{d \lambda}{\pi\left(1+\lambda^{2}\right)}, \lambda \in \mathbb{R}
$$

The Fourier transform on $\mathbb{R}$ will be denoted $\widehat{\bullet}$.

LEMMA 2.10.1.

$$
\left|\left(\chi_{(0, a)}(x) e^{x}\right)^{\wedge}(\lambda)\right|^{2}=\frac{e^{2 a}+1-2 e^{a} \cos (\lambda a)}{1+\lambda^{2}}\left(\in L^{2}(\mathbb{R}, d \lambda)\right)
$$


where $d \lambda$ here means Lebesgue measure on $\mathbb{R}$.

Proof. We have

$$
\left(\chi_{(0, a)}(x) e^{x}\right)^{\wedge}(\lambda)=\int_{0}^{a} e^{-i \lambda x} e^{x} d x=\frac{e^{(1-i \lambda) a}-1}{1-i \lambda}
$$

and (2.10.3) follows.

Now return to the RKHS $\mathscr{H}_{F}$, defined from the function $F$ in $(2.10 .1)$. The set

$$
\left\{F_{\varphi} \mid \varphi \in C_{c}^{\infty}(0, a)\right\}
$$

spans a dense subspace in $\mathscr{H}_{F}$ where

$$
F_{\varphi}(x)=\int_{0}^{a} \varphi(y) e^{-|x-y|} d y
$$

LEMMA 2.10.2. We have

$$
\begin{aligned}
\left\|F_{\varphi}\right\|_{\mathscr{H}_{F}}^{2} & =\int_{0}^{a} \int_{0}^{a} \overline{\varphi(y)} \varphi(x) e^{-|x-y|} d x d y \\
& =\int_{-\infty}^{\infty}|\widehat{\varphi}(\lambda)|^{2} \frac{d \lambda}{\pi\left(1+\lambda^{2}\right)}
\end{aligned}
$$

Proof. This follows from (2.10.2), and the fact that $F$ in (2.10.1) is the restriction of $\left(e^{-|x|}, x \in \mathbb{R}\right)$. Hence (2.10.5) follows from Parseval's identity.

We note that, if $\xi:(0, a) \rightarrow \mathbb{C}$ is any given continuous function, then $\xi$ is in the RKHS $\mathscr{H}_{F}$ if and only if

$$
\left(\lambda \mapsto\left(\chi_{(0, a)}(x) \xi(x)\right)^{\wedge}(\lambda)\right) \in L^{2}(\mathbb{R}, d \mu(\lambda))
$$

where $d \mu(\lambda)$ is the measure in (2.10.2).

LEMMA 2.10.3. Let $\Omega=(0, a), 0<a<\infty$, and let $F$ be a continuous positive definite function on $\Omega-\Omega=(-a, a)$ such that $F(0)=1$. We know that $F$ always has an extension, i.e., Ext $(F) \neq \phi$, so that there is a probability measure $\mu$ on $\mathbb{R}$ such that

$$
F(x)=\widehat{d \mu}(x), \forall x \in(-a, a) .
$$

Suppose further that $\mu$ is absolutely continuous, i.e., $\exists \Phi \in L^{1}(\mathbb{R}, d \lambda)$ such that

$$
d \mu(\lambda)=\Phi(\lambda) d \lambda .
$$

Now consider the operator

$$
D^{(F)}\left(F_{\varphi}\right)=F_{\varphi^{\prime}}
$$

on the dense domain $\left\{F_{\varphi} \mid \varphi \in C_{c}^{\infty}(0, a)\right\} \subseteq \mathscr{H}_{F}$. Then this operator $D^{(F)}$ in (2.10.9) has indices $(0,0)$, or $(1,1)$.

Its indices are $(1,1)$ if and only if

$$
\int_{-\infty}^{\infty} \frac{e^{2 a}+1-2 e^{a} \cos (\lambda a)}{1+\lambda^{2}} \Phi(\lambda) d \lambda<\infty .
$$

PRoOF. This follows from the considerations above, using (2.10.3) and (2.10.5), but applied to $(2.10 .8)$. 
Lemma 2.10.4. Fix $a, 0<a<\infty$, and let $F(\cdot):=\left.e^{-|\cdot|}\right|_{(-a, a)}$ as in (2.10.1). For all $0 \leq x_{0} \leq a$, let

$$
F_{x_{0}}(x):=\left.F\left(x-x_{0}\right)\right|_{(0, a)}(\in C(0, a)) .
$$

With

$$
\begin{aligned}
& D E F^{+}=\left\{\xi:\left(D^{(F)}\right)^{*} \xi=\xi\right\}=\operatorname{span}\left\{\xi_{+}(x):=\left.e^{-x}\right|_{(0, a)}\right\} \\
& D E F^{-}=\left\{\xi:\left(D^{(F)}\right)^{*} \xi=-\xi\right\}=\operatorname{span}\left\{\xi_{-}(x):=\left.e^{-a} e^{+x}\right|_{(0, a)}\right\}
\end{aligned}
$$

we get

$$
\left\|\xi_{+}\right\|_{\mathscr{H}_{F}}^{2}=\left\|\xi_{-}\right\|_{\mathscr{H}_{F}}^{2}=1
$$

Proof. Note that $x=0$ and $x=a$ are the endpoints in the open interval $(0, a)$ :

$$
\begin{aligned}
& \xi_{+}(x)=\left.e^{-x}\right|_{(0, a)}=F_{0}(x) \\
& \xi_{-}(x)=\left.e^{-a} e^{x}\right|_{(0, a)}=F_{a}(x) .
\end{aligned}
$$

Let $\psi_{n} \in C_{c}^{\infty}(0, a)$ be an approximate identity, such that

(1) $\psi_{n} \geq 0, \int \psi_{n}=1$;

(2) $\psi_{n} \rightarrow \delta_{a}$, as $n \rightarrow \infty$.

Then

$$
\xi_{-}(x)=F_{a}(x)=\lim _{n \rightarrow \infty} \int_{0}^{a} \psi_{n}(y) F_{y}(x) d y .
$$

This shows that $\xi_{-} \in \mathscr{H}_{F}$. Also,

$$
\begin{aligned}
\left\|\xi_{-}\right\|_{\mathscr{H}_{F}}^{2} & =\left\|F_{a}\right\|_{\mathscr{H}_{F}}^{2} \\
& =\lim _{n \rightarrow \infty} \frac{1}{2 \pi} \int_{-\infty}^{\infty}\left|\widehat{\psi_{n}}(y)\right|^{2} \widehat{F}(y) d y \\
& =\frac{1}{2 \pi} \int_{-\infty}^{\infty} \widehat{F}(y) d y=1 .
\end{aligned}
$$

Similarly, if instead, $\psi_{n} \rightarrow \delta_{0}$, then

$$
\xi_{+}(x)=F_{0}(x)=\lim _{n \rightarrow \infty} \int_{0}^{a} \psi_{n}(y) F_{y}(x) d y
$$

and $\left\|\xi_{+}\right\|_{\mathscr{H}_{F}}^{2}=1$.

Lemma 2.10.5. Let $F$ be as in (2.10.1). We have the following for its Fourier transform:

$$
\widehat{F}(y)=\frac{2-2 e^{-a}(\cos (a y)-y \sin (a y))}{1+y^{2}} .
$$


ProOF. Let $y \in \mathbb{R}$, then

$$
\begin{aligned}
\widehat{F}(y) & =\int_{-a}^{a} e^{i y x} e^{-|x|} d x \\
& =\int_{-a}^{0} e^{i y x} e^{x} d x+\int_{0}^{a} e^{i y x} e^{-x} d x \\
& =\frac{1-e^{-a(1+i y)}}{1+i y}+\frac{e^{i a(y+i)}-1}{-1+i y} \\
& =\frac{2-2 e^{-a}(\cos (a y)-y \sin (a y))}{1+y^{2}}
\end{aligned}
$$

which is the assertion.

REMARK 2.10.6. If $(F, \Omega)$ is such that $D^{(F)}$ has deficiency indices $(1,1)$, then by von Neumann's theory, the family of selfadjoint extensions is characterized by

$$
\begin{aligned}
& \operatorname{dom}\left(A_{\theta}^{(F)}\right)=\left\{F_{\psi}+c\left(\xi_{+}+e^{i \theta} \xi_{-}\right): \psi \in C_{c}^{\infty}(0, a), c \in \mathbb{C}\right\} \\
& \quad A_{\theta}^{(F)}: F_{\psi}+c\left(\xi_{+}+e^{i \theta} \xi_{-}\right) \mapsto F_{i \psi^{\prime}}+c i\left(\xi_{+}-e^{i \theta} \xi_{-}\right), \text {where } i=\sqrt{-1} .
\end{aligned}
$$

Proposition 2.10.7. Fix $a>0$, and set $\Omega=(0, a)$, so $\Omega-\Omega=(-a, a)$. Let $F:(-a, a) \rightarrow \mathbb{C}$ be positive definite and continuous, $F(0)=1$; let $D^{(F)}$ be the corresponding skew-Hermitian, i.e., $D^{(F)}\left(F_{\varphi}\right)=F_{\varphi^{\prime}}$, for all $\varphi \in C_{c}^{\infty}(0, a)$. We now assume that $D^{(F)}$ has a skew-adjoint extension (in the RKHS $\mathscr{H}_{F}$ ) which has simple and purely atomic spectrum, say $\left\{i \lambda_{n}\right\}_{n \in \mathbb{N}}, \lambda_{n} \in \mathbb{R}$. Then the complex exponentials

$$
e_{\lambda_{n}}(x)=e^{i \lambda_{n} x}
$$

are in $\mathscr{H}_{F}$, and they are orthogonal in $\mathscr{H}_{F}$; and total.

Proof. By the assumptions, we may pick some skew-adjoint extension (in $\left.\mathscr{H}_{F}\right)$, say $A$,

$$
D^{(F)} \subseteq A \subseteq-\left(D^{(F)}\right)^{*}
$$

Set $U_{A}(t):=e^{t A}, t \in \mathbb{R}$, and recall that $U_{A}(t): \mathscr{H}_{F} \rightarrow \mathscr{H}_{F}$ is a unitary representation of $\mathbb{R}$, acting on $\mathscr{H}_{F}$. By the assumption on its spectrum, we may find an orthonormal basis (ONB) $\left\{\xi_{n}\right\}$ in $\mathscr{H}_{F}$, such that

$$
U_{A}(t)=\sum_{n \in \mathbb{N}} e^{i t \lambda_{n}}\left|\xi_{n}\right\rangle\left\langle\xi_{n}\right|, t \in \mathbb{R}
$$

where $\left|\xi_{n}\right\rangle\left\langle\xi_{n}\right|$ is Dirac's term for the rank-1 projection onto $\mathbb{C} \xi_{n}$ in $\mathscr{H}_{F}$.

From (2.10.16), we get that if $0<t<a$, then

$$
U_{A}(t) F_{0}=F_{t}
$$

and by (2.10.17), therefore (with $0<t<a$ ):

$$
F_{t}(\cdot)=\sum_{n \in \mathbb{N}} e^{i t \lambda_{n}} \overline{\xi_{n}(0)} \xi_{n}(\cdot) \text { on } \Omega .
$$

We have used: $\left|\xi_{n}\right\rangle\left\langle\xi_{n}\right|\left(F_{0}\right)=\overline{\xi_{n}(0)} \xi_{n} \in \mathscr{H}_{F}$. We are also using the reproducing property in $\mathscr{H}_{F}$.

Now fix $n \in \mathbb{N}$, and take the inner-product $\left\langle\xi_{n}, \cdot\right\rangle_{\mathscr{H}_{F}}$ on both sides in (2.10.19). Using again the reproducing property, we get

$$
\xi_{n}(t)=e^{i t \lambda_{n}} \overline{\xi_{n}(0)}, t \in \Omega ;
$$


which is the desired conclusion.

Note that this makes the functions $\left\{e_{\lambda_{n}}\right\}_{n \in \mathbb{N}}$ in (2.10.15) orthogonal, and total in $\mathscr{H}_{F}$; but they are not normalized; in fact, one checks from (2.10.20) that

$$
\left\|e_{\lambda_{n}}(\cdot)\right\|_{\mathscr{H}_{F}}=\frac{1}{\left|\xi_{n}(0)\right|} \text {. }
$$

Proposition 2.10.8. For $F(x)=\left.e^{-|x|}\right|_{(-a, a)}$, the complex exponentials $e_{\lambda}$ are NOT in the corresponding RKHS $\mathscr{H}_{F}$.

Proof. We check that $e_{0} \equiv \mathbf{1}$, i.e., the constant function, is not in $\mathscr{H}_{F}$. The general case is similar.

Suppose $1 \in \mathscr{H}_{F}$. Recall the isometric isomorphic $T: \mathscr{H}_{F} \rightarrow L^{2}(\mathbb{R}, \mu)$, with

$$
d \mu(\lambda)=\frac{d \lambda}{\pi\left(1+\lambda^{2}\right)}
$$

and $T\left(F_{\varphi}\right)=\widehat{\varphi}$, for all $\varphi \in C_{c}(0, a) ; T^{*}(f)=(f d \mu)^{\vee}$, for all $f \in L^{2}(\mu)$. Also note that $R_{T^{*}}=\left(N_{T}\right)^{\perp}=\mathscr{H}_{F}$, since $T$ is isometric. So, if $\mathbf{1}=e_{0} \in \mathscr{H}_{F}$, then $\exists f \in L^{2}(\mathbb{R}, \mu)$ s.t.

$$
T^{*} f=\mathbf{1} \Longleftrightarrow \int_{\mathbb{R}} e^{-i \lambda x} \frac{f(\lambda)}{\pi\left(1+\lambda^{2}\right)} d \lambda \equiv 1, \forall x \in(0, a) .
$$

But there is only a distribution solution $f$ to $(2.10 .22)$; it is $f(\lambda):=\delta(\lambda-0)=$ Dirac mass at $\lambda=0$, so that

$$
\int_{\mathbb{R}} e^{i \lambda x} \frac{\delta(\lambda-0)}{\pi\left(1+\lambda^{2}\right)} d \lambda \equiv 1, \forall x
$$

and since $f(\lambda)=\delta(\lambda-0)$ is not in $L^{2}(\mathbb{R}, \mu)$ it follows that $\mathbf{1} \notin \mathscr{H}_{F}$.

THEOREM 2.10.9. Fix an open interval $\Omega=(0, a)$ and a continuous positive definite function $F$ on $\Omega-\Omega=(-a, a)$. Let $z$ be a complex number, then $y \rightarrow e^{-z y}$ is in $\mathscr{H}_{F}$ iff $z$ is an eigenvalue for the adjoint $\left(D^{(F)}\right)^{*}$ of $D^{(F)}$. In the affirmative case the corresponding eigenspace is $\mathbb{C} e_{z}$, in particular, it has dimension one.

Proof. If $z$ is an eigenvalue for $\left(D^{(F)}\right)^{*}$ and $\xi$ in $\mathscr{H}_{F}$ is a corresponding eigenvector, then

$$
\left\langle D^{(F)} F_{\psi}, \xi\right\rangle_{F}=\left\langle F_{\psi}, z \xi\right\rangle_{F}
$$

for all $\psi$ in $C_{c}^{\infty}(\Omega)$. Hence

$$
\int_{\Omega} \psi^{\prime}(y) \xi(y) d y=\int_{\Omega} z \psi(y) \xi(y) d y
$$

for all $\psi$ in $C_{c}^{\infty}(\Omega)$. Equivalently, $\xi$ is a weak solution to

$$
-\xi^{\prime}(y)=z \xi(y)
$$

in $\Omega$. Thus $\xi(y)=$ constant $e^{-z y}$.

Conversely, suppose $\xi_{z}: y \rightarrow e^{-z y}$ is in $\mathscr{H}_{F}$. It is sufficient to show $\xi_{z}$ is in the domain of $\left(D^{(F)}\right)^{*}$. Equivalently, we must show there is a constant $C$ such that

$$
\left|\left\langle D^{(F)} F_{\psi}, \xi_{z}\right\rangle_{F}\right| \leq C\left\|F_{\psi}\right\|_{F}
$$


for all $\psi$ in $C_{c}^{\infty}(\Omega)$. But

$$
\begin{aligned}
\left|\left\langle D^{(F)} F_{\psi}, \xi_{z}\right\rangle_{F}\right| & =\left|\int_{\Omega} \overline{\psi^{\prime}(y)} \xi_{z}(y) d y\right| \\
& =\left|z \int_{\Omega} \overline{\psi(y)} \xi_{z}(y) d y\right| \\
& =|z|\left|\left\langle F_{\psi}, \xi_{z}\right\rangle_{F}\right| .
\end{aligned}
$$

Consequently, (2.10.23) follows from the Cauchy-Schwarz inequality.

CoRollary 2.10.10. If $\xi_{r+i s}(y)=e^{(r+i s) y}$ is in $\mathscr{H}_{F}$ for some real numbers $r \neq 0$ and $s$, then $\xi_{r+i s}$ is in $\mathscr{H}_{F}$ for all real numbers $r \neq 0$ and $s$.

Proof. Let $r \neq 0$ and $s$ be real numbers. Consider the deficiency spaces

$$
D E F^{r+i s}=\left\{\xi \in \mathscr{H}_{F}:\left\langle D^{(F)} F_{\psi}, \xi\right\rangle_{F}=\left\langle F_{\psi}, z \xi\right\rangle_{F}\right\} .
$$

For $r>0$ the spaces $D E F^{r+i s}$ all have the same dimension as $D E F^{+}=D E F^{1}$ and the spaces $D E F^{-r+i s}$ all have the same dimension as $D E F^{-}=D E F^{-1}$. See, e.g., [AG93] or [DS88]. But the spaces $D E F^{ \pm}$have the same dimension. By assumption one of the spaces $D^{r+i s}$ has dimension $\geq 1$. Since the deficiency spaces are eigenspaces for $\left(D^{(F)}\right)^{*}$ the rest follows from Theorem 2.10.9.

Theorem 2.10.11. Let $a \in \mathbb{R}_{+}$, and $\lambda_{1} \in \mathbb{R}$ be given. Let $F:(-a, a) \rightarrow \mathbb{C}$ be a fixed continuous positive function. Then there following two conditions (1) and (2) are equivalent:

(1) $\exists \mu_{1} \in \operatorname{Ext}(F)$ such that $\mu_{1}\left(\left\{\lambda_{1}\right\}\right)>0$; and

(2) $e_{\lambda_{1}}(x):=\left.e^{i \lambda_{1} x}\right|_{[-a, a]} \in \mathscr{H}_{F}$.

Remark 2.10.12. Assertion (1) is stating that $\lambda_{1}$ is an atom for some $\mu_{1} \in$ $\operatorname{Ext}(F)$.

Proof. The implication $(1) \Longrightarrow(2)$ is already contained in the previous discussion.

Proof of $(2) \Longrightarrow(1)$. We first consider the skew-Hermitian operator $D^{(F)}\left(F_{\varphi}\right):=$ $F_{\varphi^{\prime}}, \varphi \in C_{c}^{\infty}(0, a)$. Using an idea of M. Krein, (see [Kre46, KL14]), we may always find a Hilbert space $\mathscr{K}$, and an isometry $J: \mathscr{H} \rightarrow \mathscr{K}$, and a strongly continuous unitary one-parameter group $U_{A}(t)=e^{t A}, t \in \mathbb{R}$, with $A^{*}=-A$; $U_{A}(t)$ acting in $\mathscr{K}$, such that

$$
\begin{gathered}
J D^{(F)}=A J \text { on } \\
\operatorname{dom}\left(D^{(F)}\right)=\left\{F_{\varphi} \mid \varphi \in C_{c}^{\infty}(0, a)\right\} ;
\end{gathered}
$$

see also Theorem 2.6.5. Since

$$
e_{1}(x)=\left.e^{i \lambda_{1} x}\right|_{[-a, a]}
$$

is in $\mathscr{H}_{F}$, we can form the following measure $\mu_{1} \in \mathscr{M}_{+}(\mathbb{R})$, now given by

$$
d \mu_{1}(\lambda):=\left\|P_{A}(d \lambda) J e_{1}\right\|_{\mathscr{K}}^{2}, \lambda \in \mathbb{R},
$$

where $P_{A}(\cdot)$ is the PVM of $U_{A}(t)$, i.e.,

$$
U_{A}(t)=\int_{\mathbb{R}} e^{i t \lambda} P_{A}(d \lambda), t \in \mathbb{R} .
$$


We claim the following two assertions:

(i) $\mu_{1} \in \operatorname{Ext}(F)$; and

(ii) $\lambda_{1}$ is an atom in $\mu_{1}$, i.e., $\mu_{1}\left(\left\{\lambda_{1}\right\}\right)>0$.

This is the remaining conclusion in the theorem.

The proof of of (i) is immediate from the construction above; using the intertwining isometry $J$ from (2.10.24), and formulas (2.10.27)-(2.10.28).

To prove (ii), we need the following:

Lemma 2.10.13. Let $F, \lambda_{1}, e_{1}, \mathscr{K}, J$ and $\left\{U_{A}(t)\right\}_{t \in \mathbb{R}}$ be as above; then we have the identity:

$$
\left\langle J e_{1}, U_{A}(t) J e_{1}\right\rangle_{\mathscr{K}}=e^{i t \lambda_{1}}\left\|e_{1}\right\|_{\mathscr{H}_{F}}^{2}, \forall t \in \mathbb{R} .
$$

Proof. It is immediate from (2.10.24)-(2.10.27) that (2.10.29) holds for $t=0$. To get it for all $t$, fix $t$, say $t>0$ (the argument is the same if $t<0$ ); and we check that

$$
\frac{d}{d s}\left(\left\langle J e_{1}, U_{A}(t-s) J e_{1}\right\rangle_{\mathscr{K}}-e^{i(t-s) \lambda_{1}}\left\|e_{1}\right\|_{\mathscr{H}_{F}}^{2}\right) \equiv 0, \forall s \in(0, t) .
$$

But this, in turn, follow from the assertions above: First

$$
D_{(F)}^{*} e_{1}=D_{(F)}^{*} J^{*} J e_{1}=J^{*} A J e_{1}
$$

holds on account of (2.10.24). We get: $e_{1} \in \operatorname{dom}\left(D_{(F)}^{*}\right)$, and $D_{(F)}^{*} e_{1}=-i \lambda_{1} e_{1}$.

Using this, the verification of is (2.10.29) now immediate.

As a result, we get:

$$
U_{A}(t) J e_{1}=e^{i t \lambda_{1}} J e_{1}, \forall t \in \mathbb{R}
$$

and by $(2.10 .28)$ :

$$
P_{A}\left(\left\{\lambda_{1}\right\}\right) J e_{1}=J e_{1}
$$

where $\left\{\lambda_{1}\right\}$ denotes the desired $\lambda_{1}$-atom. Hence, by $(2.10 .27), \mu_{1}\left(\left\{\lambda_{1}\right\}\right)=\left\|J e_{1}\right\|_{\mathscr{K}}^{2}=$ $\left\|e_{1}\right\|_{\mathscr{H}_{F}}^{2}$, which is the desired conclusion in (2).

\subsection{Discussion}

Now in the $\mathbb{R}$-case, the positive definite continuous extension $\tilde{F}$ are as in (2.4.4), and

$$
\tilde{F}(t)=\int_{\mathbb{R}} e_{t}(\lambda) d \mu(\lambda)
$$

where $\mu$ is a finite measure on $\mathbb{R}$. In this case, it is much easier to specify the RKHS.

Lemma 2.11.1. $\mathscr{H}_{\tilde{F}} \simeq L^{2}(\mathbb{R}, \mu)$.

Proof. Denote $\widehat{\varphi}$ the Fourier transform of $\varphi$, and $\check{\varphi}$ be the inverse transform.

For all $\varphi \in C_{c}^{\infty}(\mathbb{R})$,

$$
\begin{aligned}
\tilde{F}_{\varphi}(x) & =\int_{\mathbb{R}} \varphi(y) \tilde{F}(y-x) d y \\
& =\int_{\mathbb{R}} \varphi(y)\left(\int e_{y-x}(\lambda) d \mu(\lambda)\right) d y
\end{aligned}
$$




$$
\begin{aligned}
& =\int \widehat{\varphi}(\lambda) \overline{e_{x}(\lambda)} d \mu(\lambda), \text { and } \\
& \left\|\tilde{F}_{\varphi}\right\|_{\mathscr{H}_{\tilde{F}}}^{2}=\int|\widehat{\varphi}(\lambda)|^{2} d \mu(\lambda)
\end{aligned}
$$

The lemma follows from the fact the $\mathscr{H}_{\tilde{F}}$ is the completion under (2.11.1) of the set of functions $\tilde{F}_{\varphi}$.

\section{Caution.}

(1) There is NOT a similar formula for the RKHS $\mathscr{H}_{F}$ of $F$ is positive definite, continuous, but only defined on $(0, \epsilon)$.

(2) There is natural isometry

$$
\begin{aligned}
\mathscr{H}_{F} & \hookrightarrow \mathscr{H}_{\tilde{F}} \\
\left(F_{\varphi}\right) & \stackrel{W}{\rightarrow}\left(\tilde{F}_{\varphi}\right), \varphi \in C_{c}^{\infty}(0, \epsilon)
\end{aligned}
$$

but the isometry $W$ in (2.11.2) may not be onto. In some cases yes, and in other cases no.

We discussed the Lie group case $G, \Omega \subset G$ open connected, $F$ continuous and positive definite on $\Omega \cdot \Omega^{-1}=\left\{x y^{-1}: x, y \in \Omega\right\}$. In this case the most easy extensions to positive definite continuous functions $\tilde{F}$ will be to $\tilde{G}$, being the simply connected covering group of $G$. For example, $G=\mathbb{T}, \tilde{G}=\mathbb{R}$.

If $G=S L_{2}(\mathbb{R})$, then $\tilde{G}$ is a 2 -fold cover. But $\mathbb{R} \rightarrow \mathbb{R} / \mathbb{Z}$ is an $\infty$ covering of $\mathbb{T}$. 


\section{CHAPTER 3}

\section{Type I v.s. Type II Extensions}

In this chapter, we identify extensions of the initially give p.d. function $F$ which are associated with operator extensions in the RKHS $\mathscr{H}_{F}$ itself (Type I), and those which require an enlargement of $\mathscr{H}_{F}$, Type II. In the case of $G=\mathbb{R}$ (the real line) some of these continuous p.d. extensions arising from the second construction involve a spline-procedure, and a theorem of G. Polya, which leads to p.d. extensions of $F$ that are symmetric around $x=0$, and convex on the left and right half-lines. Further these extensions are supported in a compact interval, symmetric around $x=0$.

\subsection{Polya Extensions}

Part of this is the construction of Polya extensions as follow: Starting with $F$ on $(-a, a)$; we create a new $F_{e x}$, p.d. on $\mathbb{R}$, such that $\left.F_{e x}\right|_{\mathbb{R}_{+}}$is convex.

In Figure 3.1.1, the slope of $L_{+}$is chosen to be $F^{\prime}(a)$; and we take the slope of $L_{-}$to be $F^{\prime}(-a)=-F^{\prime}(a)$. Recall that $F$ is defined initially only on some fixed interval $(-a, a)$. It then follows by Polya's theorem that each of these spline extensions is continuous and positive definite.

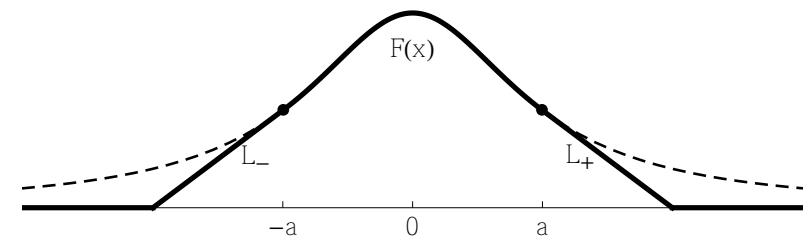

Figure 3.1.1. Spline extension of $F:(-a, a) \rightarrow \mathbb{R}$

After extending $F$ from $(-a, a)$ by adding one or more line-segments over $\mathbb{R}_{+}$, and using symmetry by $x=0$; the lines in the extensions will be such that there is a $c, 0<a<c$, and the extension $F_{\text {Polya }}(\cdot)$ satisfies $F_{\text {Polya }}(x)=0$ for all $|x| \geq c$. See Figure 3.1.2 below. 


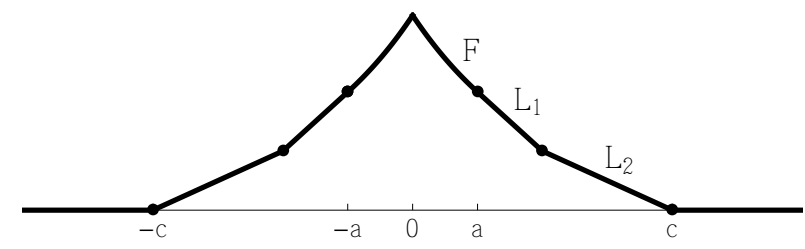

Figure 3.1.2. An example of Polya extension of $F$ on $(-a, a)$.

LEMma 3.1.1. Consider the following two functions $F_{2}(x)=1-|x|$, and $F_{3}(x)=e^{-|x|}$, each defined on a finite interval $(-a, a)$, possibly a different value of a from one to the next. The distributional double derivatives are as follows:

$$
\begin{aligned}
& F_{2}^{\prime \prime}=-2 \delta_{0} \\
& F_{3}^{\prime \prime}=F_{3}-2 \delta_{0}
\end{aligned}
$$

where $\delta_{0}$ is Dirac's delta function (i.e., point mass at $x=0, \delta_{0}=\delta(x-0)$.)

Proof. The conclusion follows from a computation, making use of L. Schwartz' theory of distributions; see [Trè06].

Proposition 3.1.2. Given $F:(-a, a) \rightarrow \mathbb{C}$, and assume $F$ has a Polya extension $F_{\text {ex }}$, then the corresponding measure $\mu_{e x} \in \operatorname{Ext}(F)$ has the following form

$$
d \mu_{e x}(\lambda)=\Phi_{e x}(\lambda) d \lambda
$$

where

$$
\Phi_{e x}(\lambda)=\frac{1}{2 \pi} \int_{-c}^{c} e^{-i \lambda y} F_{e x}(y) d y
$$

is entire analytic in $\lambda$.

Proof. An application of Fourier inversion, and the Paley-Wiener theorem.

For splines and positive definite functions, we refer to [Sch83, GSS83].

EXAmple 3.1.3 (Cauchy distribution). $F_{1}(x)=\frac{1}{1+x^{2}} ;|x|<1 . F_{1}$ is concave near $x=0$.

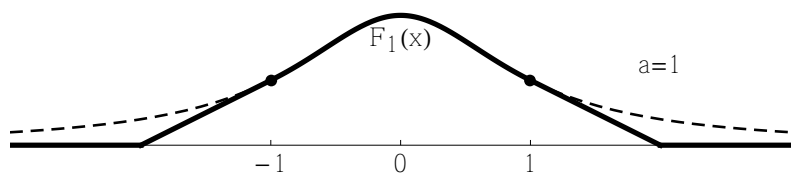

FigURE 3.1.3. Extension of $F_{1}(x)=\frac{1}{1+x^{2}} ; \Omega=(0,1)$

Example 3.1.4. $F_{2}(x)=1-|x| ;|x|<\frac{1}{2}$. Consider the following Polya extension

$$
F(x)= \begin{cases}1-|x| & \text { if }|x|<\frac{1}{2} \\ \frac{1}{3}(2-|x|) & \text { if } \frac{1}{2} \leq|x|<2 \\ 0 & \text { if }|x| \geq 2\end{cases}
$$




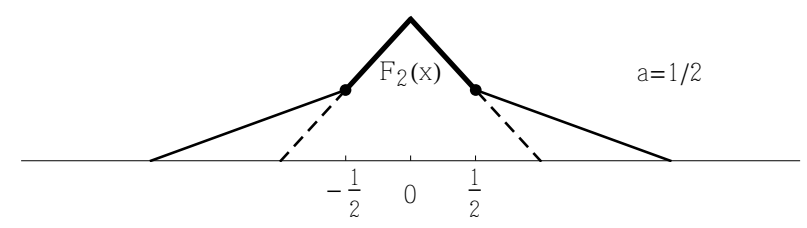

Figure 3.1.4. Extension of $F_{2}(x)=1-|x| ; \Omega=\left(0, \frac{1}{2}\right)$

This is a p.d. spline extension which is convex on $\mathbb{R}_{+}$. The corresponding measure $\mu \in \operatorname{Ext}(F)$ has the following form $d \mu(\lambda)=\Phi(\lambda) d \lambda$ where $d \lambda=$ Lebesgue measure on $\mathbb{R}$, and where

$$
\Phi(\lambda)= \begin{cases}\frac{3}{4 \pi} & \text { if } \lambda=0 \\ \frac{1}{3 \pi \lambda^{2}}(3-2 \cos (\lambda / 2)-\cos (2 \lambda)) & \text { if } \lambda \neq 0 .\end{cases}
$$

This solution is in $\operatorname{Ext}_{2}(F)$ where $F(x):=1-|x|$ for $x \in\left(-\frac{1}{2}, \frac{1}{2}\right)$. By contrast, the measure $\mu_{2}$ in Table 3.1.3 satisfies $\mu_{2} \in \operatorname{Ext}_{1}(F)$. See section 2.2.

EXAmple 3.1.5 (Ornstein-Uhlenbeck). $F_{3}(x)=e^{-|x|} ;|x|<1$. A p.d. spline extension which is convex on $\mathbb{R}_{+}$.

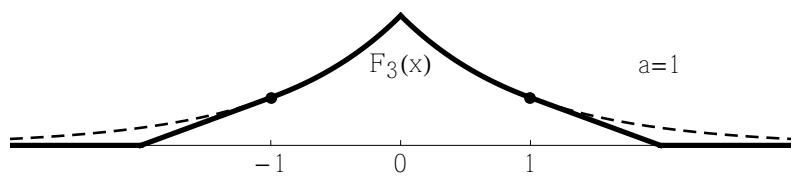

Figure 3.1.5. Extension of $F_{3}(x)=e^{-|x|} ; \Omega=(0,1)$

EXAmple 3.1.6 (Shannon). $F_{4}(x)=\left(\frac{\sin \pi x}{\pi x}\right)^{2} ;|x|<\frac{1}{2} . F_{4}$ is concave near $x=0$.

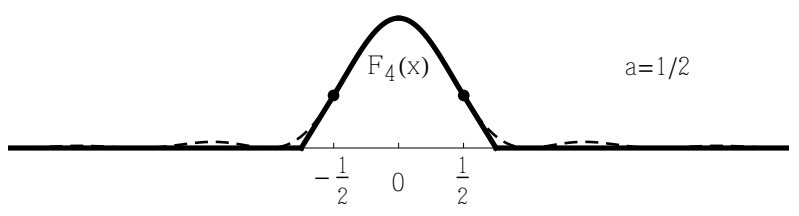

FiguRE 3.1.6. Extension of $F_{4}(x)=\left(\frac{\sin \pi x}{\pi x}\right)^{2} ; \Omega=\left(0, \frac{1}{2}\right)$

Example 3.1.7 (Gaussian distribution). $F_{5}(x)=e^{-x^{2} / 2} ;|x|<1 . \quad F_{5}$ is concave in $-1<x<1$.

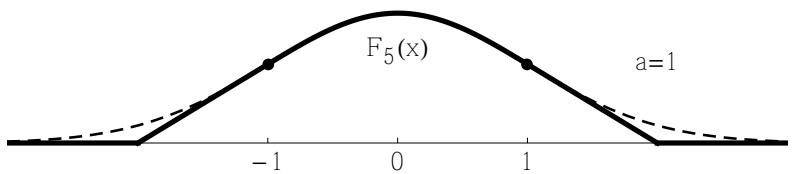

FiguRE 3.1.7. Extension $F_{5}(x)=e^{-x^{2} / 2} ; \Omega=(0,1)$ 
REMARK 3.1.8. Some spline extensions may not be positive definite. Start with a fixed continuous p.d. function $F$ on some finite interval $(-a, a)$, and consider spline extensions say $F_{e x}$ such that for some $c>a, F_{e x}(x)=0$, for $|x|>c$.

In order for Polya's theorem to be applicable, the spline extended function $F_{e x}$ to $\mathbb{R}$ must be convex on $\mathbb{R}_{+}$. By construction, then our extension to $\mathbb{R}$ is by mirror symmetry around $x=0$. So if we start with a symmetric p.d. function $F$ in $(-a, a)$ which is concave near $x=0$, then the spline extension does not satisfy the premise in Polya's theorem.

Now Polya's theorem of course only accounts for some p.d. functions, not all.

But it is not surprising that the spline extension in the list of figures 3.1.1-3.1.7 may not be p.d., e.g., Figure 3.1.3, and 3.1.6. Indeed, it is easy to check that the two partially defined functions $F$ in Figure 3.1.3, and 3.1.6 are concave near $x=0$, just calculate the double derivative $F^{\prime \prime}$.

Of the p.d. functions $F_{1}, F_{2}, \ldots F_{6}$, from table 3.1.1, we note that for the following $F_{1}, F_{4}, F_{5}$, and $F_{6}$ satisfy this: there is a $c>0$ such that the function in question is concave in the interval $[0, c]$, the value of $c$ varies from one to the next.

So these four cases from table 3.1.1 do not yield spline extensions $F_{e x}$ which are convex when restricted to $\mathbb{R}_{+}$. Polya's theorem only applies when convexity holds on $\mathbb{R}_{+}$. In that case, the spline extensions will be p.d.. And so Polya's theorem only accounts for those spline extensions $F_{e x}$ which are convex when restricted to $\mathbb{R}_{+}$. Now there may be p.d. spline extensions that are not convex when restricted to $\mathbb{R}_{+}$, and we leave open the discovery of those.

Above we introduced a special class of positive definite (p.d.) continuous extensions using a spline technique based on a theorem by Polya [Pól49]: More precisely, we extend from a fixed and finite open interval, extending a given locally defined p.d. function $F$ to the whole real line. At the endpoints of the interval we construct a linear spline extension thereby creating a real valued p.d. extension of $F$ which is symmetric around $x=0$, and is convex on the positive half-line. This spline extension is supported in a larger interval on $\mathbb{R}$ which we are free to adjust. We will refer to these p.d. extensions as Polya extensions.

For all of these examples we can use Polya's trick to generate families of continuous positive definite extensions from $(-a, a)$ to all of the real line $\mathbb{R}$. See Figure 3.1.2, 3.1.4, and 3.1.5. Some of these figures (3.1.1-3.1.8) indicate spline extensions that are not p.d..

We get the nicest p.d. extensions if we make the derivative $F^{\prime}=\frac{d F}{d x}$ a spline at the break-points. In Example 3.1.3-3.1.7, we compute $F^{\prime}(a)$. We then use symmetry for the left-hand-side of the figure:

$$
\begin{aligned}
F_{1}^{\prime}(1) & =-\frac{1}{2} \\
F_{2}^{\prime}(1 / 2) & =-1 \\
F_{3}^{\prime}(1) & =-e^{-1} \\
F_{4}^{\prime}(1 / 2) & =-16 \pi^{-2} \\
F_{5}^{\prime}(1) & =-e^{-1 / 2}
\end{aligned}
$$

So we use these slopes in making the straight line extension.

We can use Polya's theorem on each of the five functions $F_{i}, i=1, \ldots, 5$, defined initially only on $\Omega-\Omega=(-a, a)$. There we take $a=1$, or $\frac{1}{2}$, etc. 
We then get a deficiency index-problem in the RKHSs $\mathscr{H}_{F_{i}}, i=1, \ldots 5$, for the operator $D^{\left(F_{i}\right)} F_{\varphi}^{(i)}=F_{\varphi^{\prime}}^{(i)}, \forall \varphi \in C_{c}^{\infty}(0, a)$. And all the five skew-Hermitian operators in $\mathscr{H}_{F i}$ will have deficiency indices $(1,1)$.

Following is an example with deficiency indices $(0,0)$

EXAmple 3.1.9. $F_{6}(x)=\cos (x) ;|x|<\frac{\pi}{4}$

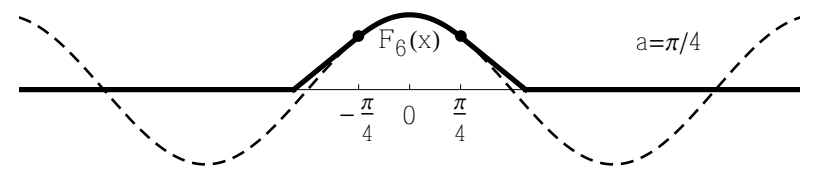

Figure 3.1.8. Extension of $F_{6}(x)=\cos (x) ; \Omega=\left(0, \frac{\pi}{4}\right)$

Lemma 3.1.10. The function $\cos x$ is positive definite on $\mathbb{R}$.

Proof. For all finite system of coefficients $\left\{c_{j}\right\}$ in $\mathbb{C}$, we have

$$
\begin{aligned}
\sum_{j} \sum_{k} \overline{c_{j}} c_{k} \cos \left(x_{j}-x_{k}\right) & =\sum_{j} \sum_{k} \overline{c_{j}} c_{k}\left(\cos x_{j} \cos x_{k}+\sin x_{j} \sin x_{k}\right) \\
& =\left|\sum_{j} c_{j} \cos x_{j}\right|^{2}+\left|\sum_{j} c_{j} \sin x_{j}\right|^{2} \geq 0 .
\end{aligned}
$$

Hence Polya also applies here, and we get more than one continuous positive definite extension from $\Omega-\Omega=\left(-\frac{\pi}{4}, \frac{\pi}{4}\right)$ to all of $\mathbb{R}$. The other extension is $x \mapsto \cos x$, for all $x \in \mathbb{R}$.

Lemma 3.1.11. $\mathscr{H}_{F_{6}}$ is 2-dimensional.

Proof. For all $\varphi \in C_{c}^{\infty}\left(0, \frac{\pi}{4}\right)$, we have:

$$
\begin{aligned}
& \int_{\Omega} \int_{\Omega} \overline{\varphi(x)} \varphi(y) F_{6}(x-y) d x d y \\
= & \left|\int_{\Omega} \varphi(x) \cos x d x\right|^{2}+\left|\int_{\Omega} \varphi(x) \sin x d x\right|^{2} \\
= & \left|\widehat{\varphi}^{(c)}(1)\right|^{2}+\left|\hat{\varphi}^{(s)}(1)\right|^{2}
\end{aligned}
$$

where $\widehat{\varphi}^{(c)}=$ the cosine-transform, and $\widehat{\varphi}^{(s)}=$ the sine-transform.

So the deficiency indices only account from some of the extension of a given positive definite function $F$ on $\Omega-\Omega$ at the starting point.

In all five examples above $\mathscr{H}_{F_{i}},(i=1, \ldots, 5)$, is infinite-dimensional; but $\mathscr{H}_{F_{6}}$ is 2-dimensional.

In the given five examples, we have p.d. continuous extensions to $\mathbb{R}$ of the following form, $\widehat{d \mu_{i}}(\cdot), i=1, \ldots, 5$, where these measures are as follows:

$$
\begin{aligned}
& d \mu_{1}(\lambda)=\frac{1}{2} e^{-|\lambda|} d \lambda \\
& d \mu_{2}(\lambda)=\left(\frac{\sin \pi \lambda}{\pi \lambda}\right)^{2} d \lambda
\end{aligned}
$$




$$
\begin{aligned}
d \mu_{3}(\lambda) & =\frac{d \lambda}{\pi\left(1+\lambda^{2}\right)} \\
d \mu_{4}(\lambda) & =\chi_{(-1,1)}(\lambda)(1-|\lambda|) d \lambda \\
d \mu_{5}(\lambda) & =\frac{1}{\sqrt{2 \pi}} e^{-\lambda^{2} / 2} d \lambda
\end{aligned}
$$

with $\lambda \in \mathbb{R}$; see Table 7.1.1 and Figure 7.1.1 below.

Corollary 3.1.12. In all five examples above, we get isometries as follows

$$
\begin{gathered}
T^{(i)}: \mathscr{H}_{F_{i}} \rightarrow L^{2}\left(\mathbb{R}, \mu_{i}\right) \\
T^{(i)}\left(F_{\varphi}^{(i)}\right)=\widehat{\varphi}
\end{gathered}
$$

for all $\varphi \in C_{c}^{\infty}(\Omega)$, where we note that $\widehat{\varphi} \in L^{2}\left(\mathbb{R}, \mu_{i}\right), i=1, \ldots, 5$; and

$$
\left\|F_{\varphi}^{(i)}\right\|_{\mathscr{H}_{F_{i}}}^{2}=\|\widehat{\varphi}\|_{L^{2}(\mu)}^{2}=\int_{\mathbb{R}}|\widehat{\varphi}|^{2} d \mu_{i}, i=1, \ldots, 5 ;
$$

but note that $T^{(i)}$ is only isometric into $L^{2}\left(\mu_{i}\right)$.

For the adjoint operator:

$$
\left(T^{(i)}\right)^{*}: L^{2}\left(\mathbb{R}, \mu_{i}\right) \rightarrow \mathscr{H}_{F_{i}}
$$

we have

$$
\left(T^{(i)}\right)^{*} f=\left(f d \mu_{i}\right)^{\vee}, \forall f \in L^{2}\left(\mathbb{R}, \mu_{i}\right) .
$$

Here is an infinite-dimensional example as a version of $F_{6}$. Fix some positive $p, 0<p<1$, and set

$$
\prod_{n=1}^{\infty} \cos \left(2 \pi p^{n} x\right)=F_{p}(x)
$$

then this is a continuous positive definite function on $\mathbb{R}$, and the law is the corresponding Bernoulli measure $\mu_{p}$ satisfying $F_{p}=\widehat{d \mu_{p}}$. Note that some of those measures $\mu_{p}$ are fractal measures.

For fixed $p \in(0,1)$, the measure $\mu_{p}$ is the law of the following random power series

$$
X_{p}(w):=\sum_{n=1}^{\infty}( \pm) p^{n}
$$

where $w \in \prod_{1}^{\infty}\{ \pm 1\}$ (= infinite Cartesian product) and where the distribution of each factor is $\left\{-\frac{1}{2}, \frac{1}{2}\right\}$, and statically independent. For relevant references on random power series, see [Neu13, Lit99].

The extensions we generate with the application of Polya's theorem are realized in a bigger Hilbert space. The deficiency indices are computed for the RKHS $\mathscr{H}_{F}$, i.e., for the "small" p.d. function $F: \Omega-\Omega \rightarrow \mathbb{C}$.

EXAmple 3.1.13. $F_{6}$ on $\left(-\frac{\pi}{4}, \frac{\pi}{4}\right)$ has the obvious extension $\mathbb{R} \ni x \rightarrow \cos x$, with a 2-dimensional Hilbert space; but the other p.d. extensions (from Polya) will be in infinite-dimensional Hilbert spaces. See Figure 3.1.9.

We must make a distinction between two classes of p.d. extensions of $F$ : $\Omega-\Omega \rightarrow \mathbb{C}$ to continuous p.d. functions on $\mathbb{R}$.

Case 1. There exists a unitary representation $U(t): \mathscr{H}_{F} \rightarrow \mathscr{H}_{F}$ such that

$$
F(t)=\left\langle\xi_{0}, U(t) \xi_{0}\right\rangle_{\mathscr{H}_{F}}, t \in \Omega-\Omega
$$




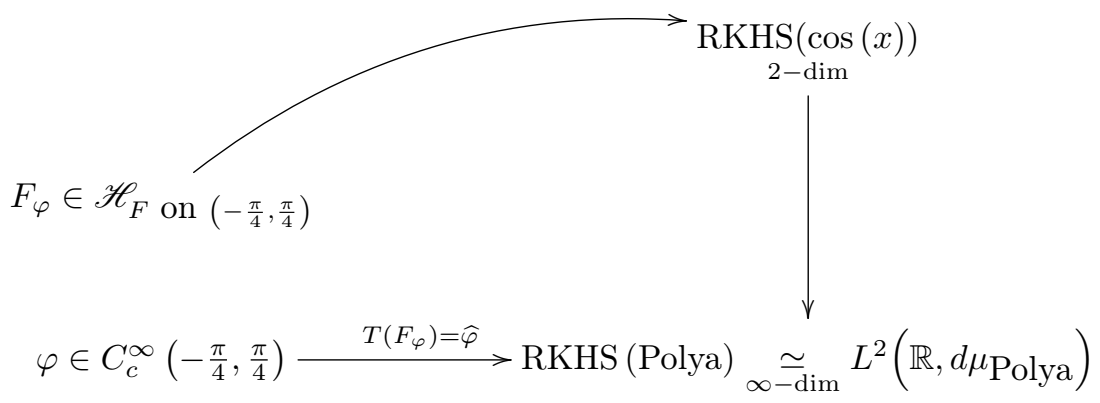

Figure 3.1.9. $\operatorname{dim}($ RKHS $(\cos x$ on $\mathbb{R}))=2$; but RKHS(Polya ext. to $\mathbb{R})$ is $\infty$-dimensional.

Case 2. (e.g., Polya extension) There exist a Hilbert space $\mathscr{K}$, and an isometry $J: \mathscr{H}_{F} \rightarrow \mathscr{K}$, and a unitary representation $U(t): \mathscr{K} \rightarrow \mathscr{K}$, such that

$$
F(t)=\left\langle J \xi_{0}, U(t) J \xi_{0}\right\rangle_{\mathscr{K}}, t \in \Omega-\Omega
$$

In both cases, $\xi_{0}=F(0-\cdot) \in \mathscr{H}_{F}$.

In case 1 , the unitary representation is realized in $\mathscr{H}_{(F, \Omega-\Omega)}$, while, in case 2 , the unitary representation $U(t)$ lives in the expanded Hilbert space $\mathscr{K}$.

Note that the RHS in both (3.1.3) and (3.1.4) is defined for all $t \in \mathbb{R}$.

Lemma 3.1.14. Let $F_{\text {ex }}$ be one of the Polya extensions if any. Then by the Galfand-Naimark-Segal (GNS) construction applied to $F_{\text {ext }}: \mathbb{R} \rightarrow \mathbb{R}$, there is a Hilbert space $\mathscr{K}$ and a vector $v_{0} \in \mathscr{K}$ and a unitary representation $\{U(t)\}_{t \in \mathbb{R}}$; $U(t): \mathscr{K} \rightarrow \mathscr{K}$, such that

$$
F_{\text {ex }}(t)=\left\langle v_{0}, U(t) v_{0}\right\rangle_{\mathscr{K}}, \forall t \in \mathbb{R} .
$$

Setting $J: \mathscr{H}_{F} \rightarrow \mathscr{K}, J \xi_{0}=v_{0}$, then $J$ defines (by extension) an isometry such that

$$
U(t) J \xi_{0}=J(\text { local translation in } \Omega)
$$

holds locally (i.e., for t sufficiently close to 0.)

Moreover, the function

$$
\mathbb{R} \ni t \mapsto U(t) J \xi_{0}=U(t) v_{0}
$$

is compactly supported.

Proof. The existence of $\mathscr{K}, v_{0}$, and $\{U(t)\}_{t \in \mathbb{R}}$ follows from the GNS-construction.

The conclusions in (3.1.6) and (3.1.7) follow from the given data, i.e., $F$ : $\Omega-\Omega \rightarrow \mathbb{R}$, and the fact that $F_{e x}$ is a spline-extension, i.e., it is of compact support; but by (3.1.5), this means that (3.1.7) is also compactly supported.

Example 3.1.4 gives a p.d. $F$ in $\left(-\frac{1}{2}, \frac{1}{2}\right)$ with $D^{(F)}$ of index $(1,1)$ and explicit measures in $\operatorname{Ext}_{1}(F)$ and in $\operatorname{Ext}_{2}(F)$.

We have the following:

Deficiency $(0,0)$ : The p.d. extension of type 1 is unique; see (3.1.3); but there may still be p.d. extensions of type 2; see (3.1.4).

Deficiency $(1,1)$ : This is a one-parameter family of extensions of type 1 ; and some more p.d. extensions are type 2 . 


\begin{tabular}{|l|c|c|}
\hline$F:(-a, a) \rightarrow \mathbb{C}$ & Indices & The Operator $D^{(F)}$ \\
\hline \hline$F_{1}(x)=\frac{1}{1+x^{2}},|x|<1$ & $(0,0)$ & $D^{(F)}$ unbounded, skew-adjoint \\
\hline$F_{2}(x)=1-|x|,|x|<\frac{1}{2}$ & $(1,1)$ & $D^{(F)}$ has unbounded sk. adj. extensions \\
\hline$F_{3}(x)=e^{-|x|},|x|<1$ & $(1,1)$ & $D^{(F)}$ has unbounded sk. adj. extensions \\
\hline$F_{4}(x)=\left(\frac{\sin \pi x}{\pi x}\right)^{2},|x|<\frac{1}{2}$ & $(0,0)$ & $D^{(F)}$ bounded, skew-adjoint \\
\hline$F_{5}(x)=e^{-x^{2} / 2},|x|>1$ & $(0,0)$ & $D^{(F)}$ unbounded, skew-adjoint \\
\hline$F_{6}(x)=\cos x,|x|<\frac{\pi}{4}$ & $(0,0)$ & $D^{(F)}$ is rank-one, dim $\left(\mathscr{H}_{F_{6}}\right)=2$ \\
\hline
\end{tabular}

TABLE 3.1.1. The deficiency indices of $D^{(F)}: F_{\varphi} \mapsto F_{\varphi^{\prime}}$ in examples 3.1.3-3.1.9
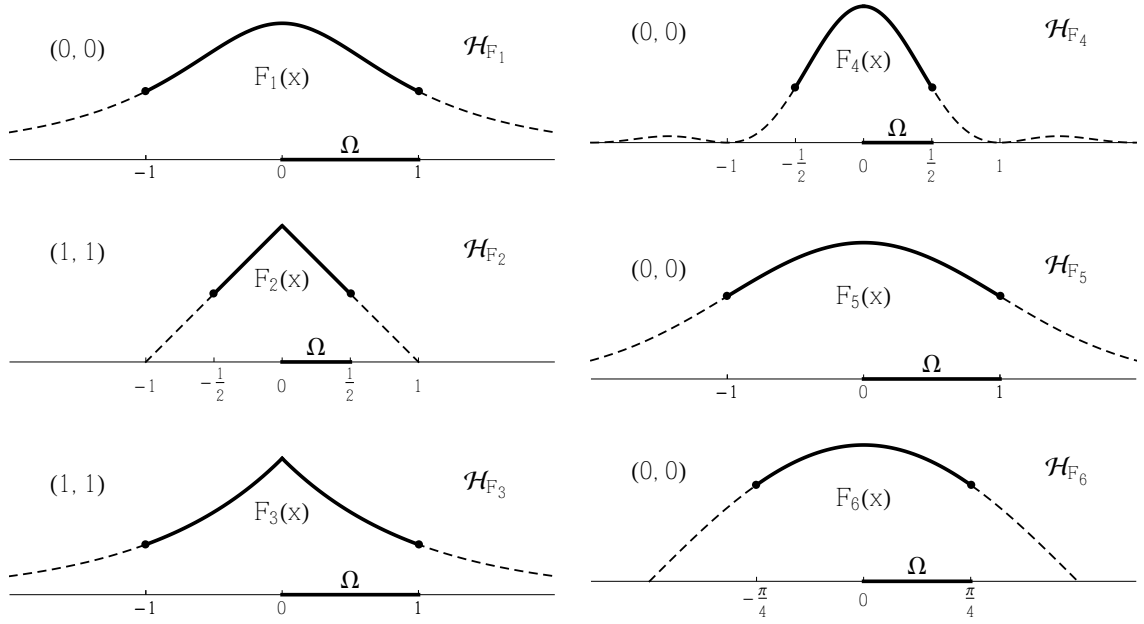

TABLE 3.1.2. Type II extensions. Six cases of p.d. continuous functions $F_{i}$ defined on a finite interval $(-a, a)$.

So we now divide

$$
\operatorname{Ext}(F)=\{\mu \in \operatorname{Prob}(\mathbb{R}) \mid \widehat{d \mu} \text { is an extension of } F\}
$$

up in subsets

$$
\operatorname{Ext}(F)=\operatorname{Ext}_{\text {type1 }}(F) \cup \operatorname{Ext}_{\text {type2 }}(F) ;
$$

where $\operatorname{Ext}_{\text {type } 2}(F)$ corresponds to the Polya extensions. 


\begin{tabular}{|l|l|}
\hline$d \mu_{1}(\lambda)=\frac{1}{2} e^{-|\lambda|} d \lambda$ & $d \mu_{4}(\lambda)=\chi_{(-1,1)}(\lambda)(1-|\lambda|) d \lambda$, cpt. support \\
\hline$d \mu_{2}(\lambda)=\left(\frac{\sin \pi \lambda}{\pi \lambda}\right)^{2} d \lambda$, Shannon & $d \mu_{5}(\lambda)=\frac{1}{\sqrt{2 \pi}} e^{-\lambda^{2} / 2} d \lambda$, Gaussian \\
\hline$d \mu_{3}(\lambda)=\frac{d \lambda}{\pi\left(1+\lambda^{2}\right)}$, Cauchy & $d \mu_{6}(\lambda)=\frac{1}{2}\left(\delta_{1}+\delta_{-1}\right)$, atomic; two Dirac masses \\
\hline
\end{tabular}

TABLE 3.1.3. The canonical isometric embeddings: $\mathscr{H}_{F_{i}} \hookrightarrow$ $L^{2}\left(\mathbb{R}, d \mu_{i}\right), i=1, \ldots, 6$.

Return to a continuous p.d. function $F:(-a, a) \rightarrow \mathbb{C}$, we take for the RKHS $\mathscr{H}_{F}$, and the skew-Hermitian operator

$$
D\left(F_{\varphi}\right)=F_{\varphi^{\prime}}, \varphi^{\prime}=\frac{d \varphi}{d x}
$$

If $D \subseteq A, A^{*}=-A$ in $\mathscr{H}_{F}$ then there exists an isometry $J: \mathscr{H}_{F} \rightarrow L^{2}(\mathbb{R}, \mu)$, where $d \mu(\cdot)=\left\|P_{U}(\cdot) \xi_{0}\right\|^{2}$,

$$
U_{A}(t)=e^{t A}=\int_{\mathbb{R}} e^{i t \lambda} P_{U}(d \lambda),
$$

$\xi_{0}=F(\cdot-0) \in \mathscr{H}_{F}, J \xi_{0}=1 \in L^{2}(\mu)$.

\subsection{The Example 3.1.5}

Here, we study Example 3.1.5, and compute the spectral date of the corresponding Mercer operator. Recall that

$$
F(x):= \begin{cases}e^{-|x|} & |x|<1 \\ e^{-1}(2-|x|) & 1 \leq|x|<2 \\ 0 & |x| \geq 2\end{cases}
$$

See Figure 3.2.1 below.

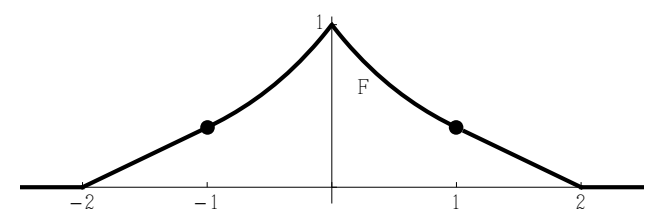

Figure 3.2.1. A Polya (spline) extension of $F_{3}=e^{-|x|} ; \Omega=(-1,1)$.

The distributional derivative of $F$ satisfies

$$
\begin{aligned}
F^{\prime \prime} & =F-2 \delta_{0}+e^{-1}\left(\delta_{2}+\delta_{-2}\right) \\
& \Uparrow \\
(1-\triangle) F & =2 \delta_{0}-e^{-1}\left(\delta_{2}+\delta_{-2}\right)
\end{aligned}
$$

This can be verified directly, using Schwartz' theory of distributions. See lemma 3.1.1 and [Trè06]. 
Thus, for

$$
F_{x}(\cdot):=F(x-\cdot)
$$

we have the translation

$$
(1-\triangle) F_{x}=2 \delta_{x}-e^{-1}\left(\delta_{x-2}+\delta_{x+2}\right)
$$

Consider the Mercer operator (see section 4.1):

$$
L^{2}(0,2) \ni g \longmapsto \int_{0}^{2} F_{x}(y) g(y) d y=\left\langle F_{x}, g\right\rangle \text {. }
$$

Suppose $\lambda \in \mathbb{R}$, and

$$
\left\langle F_{x}, g\right\rangle=\lambda g(x) .
$$

Applying $(1-\triangle)$ to both sides of $(3.2 .2)$, we get

$$
\left\langle(1-\triangle) F_{x}, g\right\rangle=\lambda\left(g(x)-g^{\prime \prime}(x)\right), 0<x<2 .
$$

By (3.2.1), we also have

$$
\left\langle(1-\triangle) F_{x}, g\right\rangle=2 g(x), 0<x<2 ;
$$

using the fact the two Dirac masses in (3.2.1), i.e., $\delta_{x \pm 2}$, are supported outside the open interval $(0,2)$.

Therefore, combining (3.2.3)-(3.2.4), we have

$$
g^{\prime \prime}=\frac{\lambda-2}{\lambda} g, \forall x \in(0,2) .
$$

By Mercer's theorem, $0<\lambda<2$. Setting

$$
k:=\sqrt{\frac{2-\lambda}{\lambda}}\left(\Leftrightarrow \lambda=\frac{2}{1+k^{2}}\right)
$$

we have

$$
g^{\prime \prime}=-k^{2} g, \forall x \in(0,2)
$$

Boundary conditions:

In $(3.2 .3)$, set $x=0$, and $x=2$, we get

$$
\begin{aligned}
& 2 g(0)-e^{-1} g(2)=\lambda\left(g(0)-g^{\prime \prime}(0)\right) \\
& 2 g(2)-e^{-1} g(0)=\lambda\left(g(2)-g^{\prime \prime}(2)\right)
\end{aligned}
$$

Now, assume

$$
g(x)=A e^{i k x}+B e^{-i k x}
$$

where

$$
\begin{aligned}
g(0) & =A+B \\
g(2) & =A e^{i k 2}+B e^{-i k 2} \\
g^{\prime \prime}(0) & =-k^{2}(A+B) \\
g^{\prime \prime}(2) & =-k^{2}\left(A e^{i k 2}+B e^{-i k 2}\right) .
\end{aligned}
$$

Therefore, for (3.2.5), we have

$$
\begin{aligned}
2(A+B)-e^{-1}\left(A e^{i k 2}+B e^{-i k 2}\right) & =\lambda\left(1+k^{2}\right)(A+B) \\
& =2(A+B)
\end{aligned}
$$

i.e.,

$$
A e^{i k 2}+B e^{-i k 2}=0 \text {. }
$$


Now, from (3.2.6) and using (3.2.7), we have

$$
A=-B \text {. }
$$

Combining (3.2.7)-(3.2.8), we conclude that

$$
\sin 2 k=0 \Longleftrightarrow k=\frac{\pi n}{2}, n \in \mathbb{Z} ;
$$

i.e.,

$$
\lambda_{n}:=\frac{2}{1+\left(\frac{n \pi}{2}\right)^{2}}, n \in \mathbb{N} .
$$

The associated ONB in $L^{2}(0,2)$ is

$$
\xi_{n}(x)=\sin \left(\frac{n \pi x}{2}\right), n \in \mathbb{N} .
$$

And the corresponding ONB in $\mathscr{H}_{F}$ consists of the functions $\left\{\sqrt{\lambda_{n}} \xi_{n}\right\}_{n \in \mathbb{N}}$, i.e.,

$$
\left\{\frac{\sqrt{2}}{\left(1+\left(\frac{n \pi}{2}\right)^{2}\right)^{1 / 2}} \sin \left(\frac{n \pi x}{2}\right)\right\}_{n \in \mathbb{N}}
$$




\section{CHAPTER 4}

\section{The Computation of $\operatorname{Ext}_{1}(F)$}

Given a continuous positive definite function $F$ on the open interval $(-1,1)$. We are concerned with the set $\operatorname{Ext}(F)$ of its extensions to continuous positive definite function defined on all of $\mathbb{R}$, as well as a certain subset $\operatorname{Ext}_{1}(F)$ of $\operatorname{Ext}(F)$. Since every such p.d. extension of $F$ is a Bochner transform of a unique positive and finite Borel measure on $\mathbb{R}$, we will speak of the set $\operatorname{Ext}(F)$ as a set of finite positive Borel measure on $\mathbb{R}$. The purpose of this chapter is to gain insight into the nature and properties of $\operatorname{Ext}_{1}(F)$ of $\operatorname{Ext}(F)$.

In section 4.1, we study a certain trace class integral operator (the Mercer operator $T_{F}$ ). We use it to identify natural Bessel frame in the RKHS $\mathscr{H}_{F}$; and we further introduce a notion of Shannon sampling of finite Borel measures on $\mathbb{R}$. We then use this in Corollary 4.1.16 to give a necessary and sufficient condition for a given finite Borel measure $\mu$ to fall in the convex set $\operatorname{Ext}(F)$ : The measures in $\operatorname{Ext}(F)$ are precisely those whose Shannon sampling recover the given p.d. function $F$ on the interval $(-1,1)$.

\subsection{Mercer Operators, and Bessel Frames}

In the considerations below, we shall be primarily concerned with the case when a fixed continuous p.d. function $F$ is defined on a finite interval $(-a, a) \subset \mathbb{R}$. In this case, by a Mercer operator, we mean an operator $T_{F}$ in $L^{2}(0, a)$ where $L^{2}(0, a)$ is defined from Lebesgue measure on $(0, a)$. We have

$$
\left(T_{F} \varphi\right)(x)=\int_{0}^{a} \varphi(y) F(x-y) d y, \forall \varphi \in L^{2}(0, a), \forall x \in(0, a) .
$$

LEMMA 4.1.1. Under the assumptions stated above, the Mercer operator $T_{F}$ is trace class in $L^{2}(0, a)$; and if $F(0)=1$, then

$$
\operatorname{trace}\left(T_{F}\right)=a \text {. }
$$

Proof. This is an application of Mercer's theorem [LP89, FR42, FM13] to the integral operator $T_{F}$ in (4.1.1). But we must check that $F$, on $(-a, a)$, extends uniquely by limit to a continuous p.d. function $F_{e x}$ on $[-a, a]$, the closed interval. This is true, and easy to verify; also see Lemma 5.0.2.

Corollary 4.1.2. Let $F$ and $(-a, a)$ be as in lemma 4.1.1. Then there is a

sequence $\left(\lambda_{n}\right)_{n \in \mathbb{N}}, \lambda_{n}>0$, s.t. $\sum_{n \in \mathbb{N}} \lambda_{n}=a$, and a system of orthogonal functions $\left\{\xi_{n}\right\} \subset L^{2}(0, a) \cap \mathscr{H}_{F}$ such that

$$
\begin{aligned}
& F(x-y)=\sum_{n \in \mathbb{N}} \lambda_{n} \xi_{n}(x) \overline{\xi_{n}(y)}, \text { and } \\
& \int_{0}^{a} \overline{\xi_{n}(x)} \xi_{m}(x) d x=\delta_{n, m}, n, m \in \mathbb{N} .
\end{aligned}
$$


Proof. An application of Mercer's theorem [LP89, FR42, FM13].

Corollary 4.1.3. Let $a \in \mathbb{R}_{+}$, and let $F:(-a, a) \rightarrow \mathbb{C}$ be positive definite and continuous, $F(0)=1$. Let $D^{(F)}$ be the skew-Hermitian operator in $\mathscr{H}_{F}$, i.e., $D^{(F)}\left(F_{\varphi}\right):=F_{\varphi^{\prime}}, \forall \varphi \in C_{c}^{\infty}(0, a)$. Let $z \in \mathbb{C} \backslash\{0\}$, and $D E F_{F}(z) \subset \mathscr{H}_{F}$ be the corresponding defect space.

Let $\left\{\xi_{n}\right\}_{n \in \mathbb{N}} \subset \mathscr{H}_{F} \cap L^{2}(\Omega), \Omega:=(0, a) ;$ and $\left\{\lambda_{n}\right\}_{n \in \mathbb{N}}$ s.t. $\lambda_{n}>0$, and $\sum_{n=1}^{\infty} \lambda_{n}=a$, be one Mercer-system as in Corollary 4.1.2.

Then $D E F_{F}(z) \neq 0$ if and only if

$$
\sum_{n=1}^{\infty} \frac{1}{\lambda_{n}}\left|\int_{0}^{a} \overline{\xi_{n}(x)} e^{z x} d x\right|^{2}<\infty .
$$

Proof. We saw in Corollary 4.1.2 that if $\left\{\xi_{n}\right\}_{n \in \mathbb{N}},\left\{\lambda_{n}\right\}_{n \in \mathbb{N}}$, is a Mercer system (i.e., the spectral data for the Mercer operator $T_{F}$ in $L^{2}(0, a)$ ), then $\xi_{n} \in \mathscr{H}_{F} \cap$ $L^{2}(\Omega)$; and $\left(\sqrt{\lambda_{n}} \xi_{n}(\cdot)\right)_{n \in \mathbb{N}}$ is an ONB in $\mathscr{H}_{F}$.

But (4.1.5) in the corollary is merely stating that the function $e_{z}(x):=e^{z x}$ has a finite $l^{2}$-expansion relative to this ONB. The rest is an immediate application of Parseval's identity (w.r.t. this ONB.)

REMARK 4.1.4. The conclusion in the corollary applies more generally: It shows that a continuous function $f$ on $[0, a]$ is in $\mathscr{H}_{F}$ iff

$$
\sum_{n=1}^{\infty} \frac{1}{\lambda_{n}}\left|\int_{0}^{a} \overline{\xi_{n}(x)} f(x) d x\right|^{2}<\infty
$$

In Theorem 4.1.5 below, we revisit the example $F_{2}$ (in $|x|<\frac{1}{2}$ ) from Table 3.1.1. This example has a number of intriguing properties that allow us to compute the eigenvalues and the eigenvectors for the Mercer operator from Corollary 4.1.2, (so $a=\frac{1}{2}$.).

Theorem 4.1.5. Set $E(x, y)=x \wedge y=\min (x, y), x, y \in\left(0, \frac{1}{2}\right)$, and

$$
\left(T_{E} \varphi\right)(x)=\int_{0}^{\frac{1}{2}} \varphi(y) x \wedge y d y
$$

then the spectral resolution of $T_{E}$ in $L^{2}\left(0, \frac{1}{2}\right)$ (Mercer operator) is as follows:

$$
E(x, y)=\sum_{n=1}^{\infty} \frac{4}{(\pi(2 n-1))^{2}} \sin ((2 n-1) \pi x) \sin ((2 n-1) \pi y)
$$

for all $\forall(x, y) \in\left(0, \frac{1}{2}\right) \times\left(0, \frac{1}{2}\right)$.

Setting

$$
\begin{gathered}
u(x)=\left(T_{E} \varphi\right)(x), \text { for } \varphi \in C_{c}^{\infty}\left(0, \frac{1}{2}\right), \text { we get } \\
\begin{array}{c}
u^{\prime}(x)=\int_{x}^{\frac{1}{2}} \varphi(y) d y, \text { and } \\
u^{\prime \prime}(x)=-\varphi(x) ;
\end{array}
\end{gathered}
$$

moreover, $u$ satisfies the boundary condition

$$
\begin{cases}u(0) & =0 \\ u^{\prime}\left(\frac{1}{2}\right) & =0\end{cases}
$$


Note that $E(x, y)$ is a p.d. kernel, but not a positive definite function in the sense of Definition 1.5.1.

In particular, $E$ is a function of two variables, as opposed to one. The purpose of Theorem 4.1.6, and Lemmas 4.1.8, and 4.1.11 is to show that the Mercer operator $T_{F}$ defined from $F=F_{2}$ (see table 3.1.1) is a rank-1 perturbation of the related operator $T_{E}$ defined from $E(x, y)$. The latter is of significance in at least two ways: $T_{E}$ has an explicit spectral representation, and $E(x, y)$ is the covariance kernel for Brownian motion; see also Figure 4.3.2. By contrast, we show in Lemma 4.3.6 that the Mercer operator $T_{F}$ is associated with pinned Brownian motion.

The connection between the two operators $T_{F}$ and $T_{E}$ is reflecting a more general feature of boundary conditions for domains $\Omega$ in Lie groups; - a topic we consider in chapter 3 and chapter 7 below.

Rank-one perturbations play a role in spectral theory in different problems; see e.g., [Yos12, DJ10, Ion01, DRSS94, TW86].

Proof of Theorem 4.1.5. We verify directly that (4.1.9)-(4.1.10) hold.

Consider the Hilbert space $L^{2}\left(0, \frac{1}{2}\right)$. The operator $\triangle_{E}:=T_{E}^{-1}$ is a selfadjoint extension of $-\left.\left(\frac{d}{d x}\right)^{2}\right|_{C_{c}^{\infty}\left(0, \frac{1}{2}\right)}$ in $L^{2}\left(0, \frac{1}{2}\right)$; and under the ONB $f_{n}(x)=$ $2 \sin ((2 n-1) \pi x), n \in \mathbb{N}$, and the boundary condition (4.1.11), $\triangle_{E}$ is diagonalized as

We conclude that

$$
\triangle_{E} f_{n}=((2 n-1) \pi)^{2} f_{n}, n \in \mathbb{N}
$$

$$
\begin{aligned}
& \triangle_{E}=\sum_{n=1}^{\infty}((2 n-1) \pi)^{2}\left|f_{n}\right\rangle\left\langle f_{n}\right| \\
& T_{E}=\triangle_{E}^{-1}=\sum_{n=1}^{\infty} \frac{1}{((2 n-1) \pi)^{2}}\left|f_{n}\right\rangle\left\langle f_{n}\right|
\end{aligned}
$$

where $P_{n}:=\left|f_{n}\right\rangle\left\langle f_{n}\right|=$ Dirac's rank-1 projection in $L^{2}\left(0, \frac{1}{2}\right)$, and

$$
\begin{aligned}
\left(P_{n} \varphi\right)(x) & =\left\langle f_{n}, \varphi\right\rangle f_{n}(x) \\
& =\left(\int_{0}^{\frac{1}{2}} f_{n}(y) \varphi(y) d y\right) f_{n}(x) \\
& =4 \sin ((2 n-1) \pi x) \int_{0}^{\frac{1}{2}} \sin ((2 n-1) \pi y) \varphi(y) d y .
\end{aligned}
$$

Combing (4.1.14) and (4.1.15), we get

$$
\left(T_{E} \varphi\right)(x)=\sum_{n=1}^{\infty} \frac{4 \sin ((2 n-1) \pi x)}{((2 n-1) \pi)^{2}} \int_{0}^{\frac{1}{2}} \sin ((2 n-1) \pi y) \varphi(y) d y .
$$

Note the normalization considered in (4.1.7) and (4.1.16) is consistent with the condition:

$$
\sum_{n=1}^{\infty} \lambda_{n}=\operatorname{Trace}\left(T_{E}\right)
$$

in Corollary 4.1.2 for the Mercer eigenvalues $\left(\lambda_{n}\right)_{n \in \mathbb{N}}$. Indeed,

$$
\operatorname{Trace}\left(T_{E}\right)=\int_{0}^{\frac{1}{2}} x \wedge x d x=\frac{1}{8} \text { and }
$$




$$
\sum_{n=1}^{\infty} \frac{1}{((2 n-1) \pi)^{2}}=\frac{1}{8} .
$$

Theorem 4.1.6. Set $F(x-y)=1-|x-y|, x, y \in\left(0, \frac{1}{2}\right) ; K^{(E)}(x, y)=x \wedge y=$ $\min (x, y)$, and let

$$
\left(T_{E} \varphi\right)(x)=\int_{0}^{\frac{1}{2}} \varphi(y) K^{(E)}(x, y) d y
$$

then

$$
K^{(E)}(x, y)=\sum_{n=1}^{\infty} \frac{4 \sin ((2 n-1) \pi x) \sin ((2 n-1) \pi y)}{(\pi(2 n-1))^{2}}
$$

and

$$
F(x-y)=1-x-y+2 \sum_{n=1}^{\infty} \frac{4 \sin ((2 n-1) \pi x) \sin ((2 n-1) \pi y)}{(\pi(2 n-1))^{2}} .
$$

That is,

$$
F(x-y)=1-x-y+2 K^{(E)}(x, y) .
$$

REMARK 4.1.7. Note the trace normalization $\operatorname{trace}\left(T_{F}\right)=\frac{1}{2}$ holds. Indeed, from (4.1.19), we get

$$
\begin{aligned}
\operatorname{Trace}\left(T_{F}\right) & =\int_{0}^{\frac{1}{2}}\left(1-2 x+2 \sum_{n=1}^{\infty} \frac{4 \sin ^{2}((2 n-1) \pi x)}{(\pi(2 n-1))^{2}}\right) d x \\
& =\frac{1}{2}-\frac{1}{4}+2 \sum_{n=1}^{\infty} \frac{1}{(\pi(2 n-1))^{2}} \\
& =\frac{1}{2}-\frac{1}{4}+2 \cdot \frac{1}{8}=\frac{1}{2}
\end{aligned}
$$

where $\frac{1}{2}$ on the RHS is the right endpoint of the interval $\left[0, \frac{1}{2}\right]$.

Proof of Theorem 4.1.6. The theorem follows from lemma 4.1.8 and lemma 4.1.11.

Lemma 4.1.8. Consider the two integral kernels:

$$
\begin{aligned}
F(x-y) & =1-|x-y|, x, y \in \Omega ; \\
K^{(E)}(x, y) & =x \wedge y=\min (x, y), x, y \in \Omega .
\end{aligned}
$$

We take $\Omega=\left(0, \frac{1}{2}\right)$. Then

$$
\begin{gathered}
F(x-y)=2 K^{(E)}(x, y)+1-x-y ; \text { and } \\
\left(F_{x}-2 K_{x}^{(E)}\right)^{\prime \prime}(y)=-2 \delta(0-y) .
\end{gathered}
$$

Proof. A calculation yields:

$$
x \wedge y=\frac{x+y-1+F(x-y)}{2},
$$

and therefore, solving for $F(x-y)$, we get (4.1.23). 
To prove (4.1.24), we calculate the respective Schwartz derivatives (in the sense of distributions). Let $H_{x}=$ the Heaviside function at $x$, with $x$ fixed. Then

$$
\begin{aligned}
& \left(K_{x}^{(E)}\right)^{\prime}=H_{0}-H_{x}, \text { and } \\
& \left(K_{x}^{(E)}\right)^{\prime \prime}=\delta_{0}-\delta_{x}
\end{aligned}
$$

combining (4.1.26) with $\left(F_{x}\right)^{\prime \prime}=-2 \delta_{x}$, we get

$$
\left(F_{x}-2 K_{x}^{(E)}\right)^{\prime \prime}=-2 \delta_{x}-2\left(\delta_{0}-\delta_{x}\right)=-2 \delta_{0}
$$

which is the desired conclusion (4.1.24).

REMARK 4.1.9. From (4.1.23), we get the following formula for three integral operators

$$
\begin{gathered}
T_{F}=2 T_{E}+L, \text { where } \\
(L \varphi)(x)=\int_{0}^{\frac{1}{2}} \varphi(y)(1-x-y) d y .
\end{gathered}
$$

Now in Lemma 4.1.8, we diagonalize $T_{E}$, but the two Hermitian operators on the RHS in (4.1.28), do not commute. But the perturbation $L$ in (4.1.28) is still relatively harmless; it is a rank-1 operator with just one eigenfunction: $\varphi(x)=a+b x$, where $a$ and $b$ are determined from $(L \varphi)(x)=\lambda \varphi(x)$; and

$$
\begin{aligned}
(L \varphi)(x) & =(1-x) \frac{a}{2}-\frac{1}{8}\left(a+\frac{b}{3}\right) \\
& =\left(\frac{3}{8} a-\frac{b}{24}\right)-\left(\frac{a}{2}\right) x=\lambda(a+b x)
\end{aligned}
$$

thus the system of equations

$$
\left\{\begin{array}{l}
\left(\frac{3}{8}-\lambda\right) a-\frac{1}{24} b=0 \\
-\frac{1}{2} a-\lambda b=0
\end{array}\right.
$$

It follows that

$$
\begin{aligned}
\lambda & =\frac{1}{48}(9+\sqrt{129}) \\
b & =-\frac{1}{2}(\sqrt{129}-9) a .
\end{aligned}
$$

REMARK 4.1.10 (A dichotomy for integral kernel operators). Note the following dichotomy for the two integral kernel-operators, one with the kernel $L(x, y)=$ $1-x-y$, a rank-one operator; and the other $T_{F}$ corresponding to $F=F_{2}$, i.e., with kernel $F(x-y)=1-|x-y|$. And by contrast, $T_{F}$ is an infinite dimensional integral-kernel operator. Denoting both the kernel $L$, and the rank-one operator, by the same symbol, we then establish the following link between the two integral operators: The two operators $T_{F}$ and $L$ satisfy the identity $T_{F}=L+2 T_{E}$, where $T_{E}$ is the integral kernel-operator defined from the covariance function of Brownian motion. For more applications of rank-one perturbations, see e.g., [Yos12, DJ10, Ion01, DRSS94, TW86]. 
Lemma 4.1.11. Set

$$
\left(T_{E} \varphi\right)(x)=\int_{0}^{\frac{1}{2}} K^{(E)}(x, y) \varphi(y) d y, \varphi \in L^{2}\left(0, \frac{1}{2}\right), x \in\left(0, \frac{1}{2}\right)
$$

Then $s_{n}(x):=\sin ((2 n-1) \pi x)$ satisfies

$$
T_{E} s_{n}=\frac{1}{((2 n-1) \pi)^{2}} s_{n}
$$

and we have

$$
K^{(E)}(x, y)=\sum_{n \in \mathbb{N}} \frac{4}{((2 n-1) \pi)^{2}} \sin ((2 n-1) \pi x) \sin ((2 n-1) \pi y)
$$

Proof. Let $\Omega=\left(0, \frac{1}{2}\right)$. Setting

$$
s_{n}(x):=\sin ((2 n-1) \pi x) ; x \in \Omega, n \in \mathbb{N} .
$$

Using (4.1.26), we get

$$
\left(T_{E} s_{n}\right)(x)=\frac{1}{((2 n-1) \pi)^{2}} s_{n}(x), x \in \Omega, n \in \mathbb{N},
$$

where $T_{E}$ is the integral operator with kernel $K^{(E)}$, and $s_{n}$ is as in (4.1.33). Since

$$
\int_{0}^{\frac{1}{2}} \sin ^{2}((2 n-1) \pi x) d x=\frac{1}{4}
$$

the desired formula (4.1.32) holds.

Corollary 4.1.12. Let $T_{F}$ and $T_{E}$ be the integral operators in $L^{2}\left(0, \frac{1}{2}\right)$ defined in the lemmas; i.e., $T_{F}$ with kernel $F(x-y)$; and $T_{E}$ with kernel $x \wedge y$. Then the selfadjoint operator $\left(T_{F}-2 T_{E}\right)^{-1}$ is well-defined, and it is the Friedrichs extension of $-\left.\frac{1}{2}\left(\frac{d}{d x}\right)^{-1}\right|_{C_{c}^{\infty}\left(0, \frac{1}{2}\right)}$ as a Hermitian and semibounded operator in $L^{2}\left(0, \frac{1}{2}\right)$.

Proof. Formula (4.1.24) in the lemma translates into

$$
\left(T_{F} \varphi-2 T_{E} \varphi\right)^{\prime \prime}=-2 \varphi
$$

for all $\varphi \in C_{c}^{\infty}\left(0, \frac{1}{2}\right)$. Hence $\left(T_{F}-2 T_{E}\right)^{-1}$ is well-defined as an unbounded selfadjoint operator in $L^{2}\left(0, \frac{1}{2}\right)$; and

$$
\left(T_{F}-2 T_{E}\right)^{-1} \varphi=-\frac{1}{2} \varphi^{\prime \prime}, \forall \varphi \in C_{c}^{\infty}\left(0, \frac{1}{2}\right) .
$$

Since the Friedrichs extension is given by Dirichlet boundary condition in $L^{2}\left(0, \frac{1}{2}\right)$, the result follows.

Theorem 4.1.13. Let $F:(-1,1) \rightarrow \mathbb{C}$ be a fixed continuous and positive definite function, and let

$$
T_{F}: L^{2}(0,1) \rightarrow \mathscr{H}_{F}
$$

be the corresponding Mercer operator, where

- $L^{2}(0,1)=$ the $L^{2}$-space of $[0,1]$ w.r.t. Lebesgue measure restricted to $[0,1]$; and

- $\mathscr{H}_{F}$ is the RKHS from $F$. 
Let $\mu \in \operatorname{Ext}(F)$; then the range of $T_{F}$ as a subspace of $\mathscr{H}_{F}$ admits the following representation:

$$
\operatorname{Ran}\left(T_{F}\right)=\left\{\sum_{n \in \mathbb{Z}} c_{n} f_{n}(x)\right\} \text { where }
$$

- $\left(c_{n}\right) \in l^{2}(\mathbb{Z})$, i.e., $\sum_{n \in \mathbb{Z}}\left|c_{n}\right|^{2}<\infty$; and

- $\left\{f_{n} \mid n \in \mathbb{Z}\right\} \subset \mathscr{H}_{F}$ is given by

$$
f_{n}(x)=\int_{\mathbb{R}} e^{i \lambda x} \frac{\sin \pi(\lambda-n)}{\pi(\lambda-n)} e^{-i \pi(\lambda-n) / 2} d \mu(\lambda)
$$

for all $n \in \mathbb{Z}$, and all $x \in[0,1]$.

Setting

$$
\begin{gathered}
\operatorname{Sh} a(\xi)=e^{i \xi / 2} \frac{\sin \xi}{\xi}, \text { then } \\
f_{n}(x)=\int_{\mathbb{R}} \operatorname{Sh} a(\pi(\lambda-n)) e^{i \lambda x} d \mu(x) .
\end{gathered}
$$

Proof. We first show $\subseteq$ in (4.1.36). If $\varphi \in L^{2}(0,1)$, then we may apply Shannon's sampling theorem [KT09] to

$$
\widehat{\varphi}(\lambda)=\int_{0}^{1} e^{-i \lambda x} \varphi(x) d x .
$$

Hence (Shannon sampling),

$$
\begin{aligned}
\widehat{\varphi}(\lambda)= & \sum_{n \in \mathbb{Z}} \widehat{\varphi}(n) \frac{\sin \pi(\lambda-n)}{\pi(\lambda-n)} e^{-i \pi(\lambda-n) / 2} \\
= & \sum_{n \in \mathbb{Z}} \widehat{\varphi}(n) \operatorname{Sh} a(\pi(\lambda-n)) e^{-i \pi(\lambda-n) / 2} \quad \text { where } \\
& \int_{0}^{1}|\varphi(x)|^{2} d x=\sum_{n \in \mathbb{Z}}|\widehat{\varphi}(n)|^{2}
\end{aligned}
$$

where the kernel on the RKS in (4.1.41) is the Shannon integral kernel.

But we already proved that

$$
\left(T_{F} \varphi\right)(x)=\int_{\mathbb{R}} e^{i \lambda x} \widehat{\varphi}(\lambda) d \mu(\lambda)
$$

holds for all $\varphi \in L^{2}(0,1), x \in[0,1]$. Now substituting (4.1.41) into the RHS of (4.1.42), we arrive at the desired conclusion (4.1.36). The interchange of integration and summation is justified by (4.1.42) and Fubini.

It remains to prove $\supseteq$ in (4.1.36). Let $\left(c_{n}\right) \in l^{2}(\mathbb{Z})$ be given. By Parseval, it follows that

$$
\varphi(x)=\sum_{n \in \mathbb{Z}} c_{n} e^{i 2 \pi n x} \in L^{2}(0,1),
$$

and that (4.1.42) holds. Now the same argument (as above) with Fubini proves that

$$
\left(T_{F} \varphi\right)(x)=\sum_{n \in \mathbb{Z}} c_{n} f_{n}(x), x \in[0,1] .
$$




\begin{tabular}{|l|c|c|}
\hline & $\Re\left\{f_{n}\right\}(x)$ & $\Im\{f\}(x)$ \\
\hline$x=0$ & $\frac{e^{-1}+1-2}{1+(2 \pi n)^{2}}$ & $-\frac{2 \pi n\left(1-e^{-1}\right)}{1+(2 \pi n)^{2}}$ \\
\hline$x=1$ & $\frac{e^{-1}+1-2}{1+(2 \pi n)^{2}}$ & $\frac{2 \pi n\left(1-e^{-1}\right)}{1+(2 \pi n)^{2}}$ \\
\hline
\end{tabular}

TABLE 4.1.1. Boundary values of the Shannon functions, s.t. $\Re\left\{f_{n}\right\}(1)=\Re\left\{f_{n}\right\}(0)$, and $\Im\left\{f_{n}\right\}(1)=-\Im\left\{f_{n}\right\}(0)$.

EXAMPLE 4.1.14. Let $F=F_{3}$ from Table 3.1.1, i.e., $F(x):=e^{-|x|}, x \in(-1,1)$; then the generating function system $\left\{f_{n}\right\}_{n \in \mathbb{Z}}$ in $\mathscr{H}_{F}$ (from Shannon sampling) is as follows:

$$
\begin{aligned}
& \Re\left\{f_{n}\right\}(x)=\frac{e^{x-1}+e^{-x}-2 \cos (2 \pi n x)}{1+(2 \pi n)^{2}}, \text { and } \\
& \Im\left\{f_{n}\right\}(x)=\frac{\left(e^{x-1}-e^{-x}\right) 2 \pi n-2 \sin (2 \pi n x)}{1+(2 \pi n)^{2}}, \forall n \in \mathbb{Z}, x \in[0,1] .
\end{aligned}
$$

REMARK 4.1.15. While this system is explicit, it has a drawback compared to the eigenfunctions for the Mercer operator, in that $\left\{f_{n}\right\}_{n \in \mathbb{Z}}$ is not orthogonal. The boundary values are as in Table 4.1.1.

Corollary 4.1.16. Let $F:(-1,1) \rightarrow \mathbb{C}$ be continuous and p.d., $F(0)=1$, and let $\mu \in \mathscr{M}_{+}(\mathbb{R})$; then the following two conditions are equivalent:

(1) $\mu \in \operatorname{Ext}(F)$

(2) For all $x \in(-1,1)$,

$$
\sum_{n \in \mathbb{Z}} \int_{\mathbb{R}} e^{i \lambda x} \operatorname{Sh} a(\pi(\lambda-n)) d \mu(\lambda)=F(x) .
$$

Proof. In the assertion (2) on the LHS, we use "Sha" to denote Shannon's sampling kernel; see (4.1.38)-(4.1.39).

The implication $(2) \Longrightarrow(1)$ follows since $\operatorname{LHS}_{(4.1 .43)}$ is a continuous p.d. function defined on all of $\mathbb{R}$; and so it is an extension as required. It remains to show that $\operatorname{LHS}_{(4.1 .43)}=\widehat{d \mu}$ (Bochner transform), but his follows from the proof of Theorem 4.1.13.

The argument for $(1) \Longrightarrow(2)$ is as follows: Let $x \in(-1,1)$; then

$$
\begin{aligned}
F(x) & =\sum_{n \in \mathbb{Z}} \int_{\mathbb{R}} e^{i \lambda x} \operatorname{Sh} a(\pi(\lambda-n)) d \mu(\lambda) \quad\left(\text { Parseval on } L^{2}\right) \\
& =\widehat{d \mu}(x) ;
\end{aligned}
$$

which is $(2)$.

In the remaining of this section, we turn to domains in Lie groups.

REMARK 4.1.17. A trace class Mercer operator may be defined for any continuous p.d. function $F: \Omega^{-1} \cdot \Omega \rightarrow \mathbb{C}$ where $\Omega \subset G$ is a subset in a Lie group satisfying the following: 
B1. $\Omega \neq \phi$,

B2. $\Omega$ is open and connected,

B3. the closure $\bar{\Omega}$ is compact,

B4. the boundary of $\bar{\Omega}$ has Haar measure zero.

We then set

$$
\left(T_{F} \varphi\right)(x)=\int_{\Omega} \varphi(y) F\left(y^{-1} x\right) d y
$$

where $d y$ is the restriction of Haar measure on $G$ to the subset $\Omega$, or equivalently to $\bar{\Omega}$.

Note that with Lemma 5.0.2 and assumption B3 in Remark 4.1.17, we conclude that

$$
\mathscr{H}_{F} \subseteq C(\bar{\Omega}) \subset L^{2}(\Omega)
$$

so it is natural to ask: "What is the orthogonal complement of $\mathscr{H}_{F}$ in the larger Hilbert space $L^{2}(\Omega)$ ?" The answer is as follows:

Corollary 4.1.18. We have

$$
L^{2}(\Omega) \ominus \mathscr{H}_{F}=\operatorname{Ker}\left(T_{F}\right)
$$

where $T_{F}$ is the Mercer operator. As an operator in $L^{2}(\Omega), T_{F}$ takes the form:

$$
T_{F}=\left(\begin{array}{cc}
\mathscr{H}_{F} & K \operatorname{ker}\left(T_{F}\right) \\
T_{F} & 0 \\
0 & 0
\end{array}\right) \begin{gathered}
\mathscr{H}_{F} \\
\operatorname{Ker}\left(T_{F}\right)
\end{gathered}
$$

ProOF. We shall first need the following:

LEMMA 4.1.19. There is a finite constant $C_{1}$ such that

$$
\|\xi\|_{L^{2}(\Omega)} \leq C_{1}\|\xi\|_{\mathscr{H}_{F}}
$$

for all $\xi \in \mathscr{H}_{F}$.

Proof. Since $\mathscr{H}_{F} \subset L^{2}(\Omega)$ by (4.1.45), the inclusion mapping, $\mathscr{H}_{F} \rightarrow L^{2}(\Omega)$, is closed and therefore bounded; by the Closed-Graph Theorem; and so the estimate (4.1.47) follows. (See also Theorem 4.1.25 below for an explicit bound $C_{1}$.)

Proof of the Corollary continued:

Let $\xi \in \mathscr{H}_{F}$, and pick $\varphi_{n} \in C_{c}(\Omega)$ such that

$$
\left\|\xi-F_{\varphi_{n}}\right\|_{\mathscr{H}_{F}} \rightarrow 0, n \rightarrow \infty \text {. }
$$

Note $F_{\varphi_{n}}=T_{F} \varphi_{n} ;$ and we get

$$
\left\|\xi-T_{F} \varphi_{n}\right\|_{L^{2}(\Omega)} \underset{(4.1 .47)}{\leq} C_{1}\left\|\xi-F_{\varphi_{n}}\right\|_{\mathscr{H}_{F}} \underset{(4.1 .48)}{\longrightarrow} 0, n \rightarrow \infty
$$

Therefore, if $f \in L^{2}(\Omega)$ is given, we conclude that the following properties are equivalent:

$$
\begin{aligned}
\langle f, \xi\rangle_{L^{2}(\Omega)} & =0, \forall \xi \in \mathscr{H}_{F} \\
& \Uparrow \\
\left\langle f, F_{\varphi}\right\rangle_{L^{2}(\Omega)} & =0, \forall \varphi \in C_{c}(\Omega) \\
& \Uparrow \\
\left\langle f, T_{F} \varphi\right\rangle_{L^{2}(\Omega)} & =0, \forall \varphi \in C_{c}(\Omega)
\end{aligned}
$$




$$
\begin{aligned}
& \Uparrow \quad \text { (since } T_{F} \text { is selfadjoint as an operator in } L^{2}(\Omega) \text { ) } \\
& \left\langle T_{F} f, \varphi\right\rangle_{L^{2}(\Omega)}=0, \forall \varphi \in C_{c}(\Omega) \\
& \mathbb{1} \quad\left(\text { since } C_{c}(\Omega) \text { is dense in } L^{2}(\Omega)\right. \text { ) } \\
& T_{F} f=0
\end{aligned}
$$

REMARK 4.1.20. Note that $\operatorname{Ker}\left(T_{F}\right)$ may be infinite-dimensional. This happens for example, in the cases studied in section 2.8, and in the case of $F_{6}$ in Table 3.1.1 (section 3.1). On the other hand, these examples are rather degenerate since they have $\operatorname{dim} \mathscr{H}_{F}<\infty$.

Convention. When computing $T_{F}(f)$ for $f \in L^{2}(\Omega)$, we may use (4.1.45) to write $f$ as $f=f^{(F)}+f^{(K)}$, where $f^{(F)} \in \mathscr{H}_{F}$, and $f^{(K)} \in \operatorname{Ker}\left(T_{F}\right)$, and $\left\langle f^{(F)}, f^{(K)}\right\rangle_{L^{2}(\Omega)}=0$. As a result,

$$
T_{F}(f)=T_{F} f^{(F)}+T_{F} f^{(K)}=T_{F} f^{(F)},
$$

and $\operatorname{Ker}\left(T_{F}\right)$ is not a problem: For example, when we write $T_{F}^{-1}(f)$, we mean $T_{F}^{-1} f^{(F)}$.

Corollary 4.1.21. Let $G, \Omega, F$ and $\mathscr{H}_{F}$ be as above, assume that $\Omega \subset G$ satisfies B1-B4 in Remark 4.1.17. Suppose in addition that $G=\mathbb{R}^{k}$, and that there exists $\mu \in \operatorname{Ext}(F)$ such that the support suppt $(\mu)$ contains a non-empty open subset in $\mathbb{R}^{k}$; then $\operatorname{Ker}\left(T_{F}\right)=0$.

Proof. We saw in Corollary 2.4.9 that there is an isometry $\mathscr{H}_{F} \rightarrow L^{2}\left(\mathbb{R}^{k}, \mu\right)$ given on the dense subset $\left\{F_{\varphi} \mid \varphi \in C_{c}(\Omega)\right\}$, by $F_{\varphi} \mapsto \widehat{\varphi}$, s.t. $\left\|F_{\varphi}\right\|_{\mathscr{H}_{F}}=\|\widehat{\varphi}\|_{L^{2}\left(\mathbb{R}^{k}, \mu\right)}$; where $\widehat{\varphi}$ denotes Fourier transform in $\mathbb{R}^{k}$. Since $F_{\varphi}=T_{F}(\varphi)$, for all $\varphi \in C_{c}(\Omega)$, and $C_{c}(\Omega)$ is dense in $L^{2}(\Omega)$, we get

$$
\left\|T_{F} f\right\|_{\mathscr{H}_{F}}=\|\hat{f}\|_{L^{2}\left(\mathbb{R}^{k}, \mu\right)}
$$

holds also for $f \in L^{2}(\Omega)$. So $f \in \operatorname{Ker}\left(T_{F}\right) \Rightarrow \widehat{f} \equiv 0$ on $\operatorname{suppt}(\mu)$, by (4.1.49). But since $\bar{\Omega}$ is compact, we conclude by Paley-Wiener, that $\widehat{f}$ is entire analytic. Since $\operatorname{suppt}(\mu)$ consists a non-empty open set, we conclude that $\widehat{f} \equiv 0$; and by (4.1.49), that therefore $f=0$.

Theorem 4.1.22. Let $G, \Omega, F: \Omega^{-1} \Omega \rightarrow \mathbb{C}$, and $\mathscr{H}_{F}$ be as in Remark 4.1.17, i.e., we assume that B1-B4 hold.

(1) Let $T_{F}$ denote the corresponding Mercer operator $T_{F}: L^{2}(\Omega) \rightarrow \mathscr{H}_{F}$. Assume further that $\Omega$ has finite Haar measure. Then $T_{F}^{*}$ is also a densely defined operator on $\mathscr{H}_{F}$ as follows:

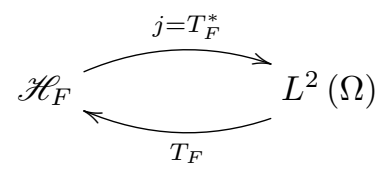

(2) Moreover, every $\xi \in \mathscr{H}_{F}$ has a realization $j(\xi)$ as a bounded uniformly continuous function on $\Omega$, and

$$
T_{F}^{*} \xi=j(\xi), \forall \xi \in \mathscr{H}_{F} .
$$


(3) Finally, the operator $j$ from (1)-(2) satisfies

$$
\operatorname{Ker}(j)=0 .
$$

Proof. We begin with the claims relating (4.1.50) and (4.1.51) in the theorem.

Using the reproducing property in the RKHS $\mathscr{H}_{F}$, we get the following two estimates, valid for all $\xi \in \mathscr{H}_{F}$ :

$$
|\xi(x)-\xi(y)|^{2} \leq 2\|\xi\|_{\mathscr{H}_{F}}^{2}\left(F(e)-\Re\left(F\left(x^{-1} y\right)\right)\right), \forall x, y \in \Omega
$$

and

$$
|\xi(x)| \leq\|\xi\|_{\mathscr{H}_{F}} \sqrt{F(e)}, \forall x \in \Omega
$$

In the remaining of the proof, we shall adopt the normalization $F(e)=1$; so the assertion in (4.1.54) states that point-evaluation for $\xi \in \mathscr{H}_{F}$ is contractive.

REMARK. In the discussion below, we give conditions which yield boundedness of the operators in (4.1.50). If bounded, then, by general theory, we have

$$
\|j\|_{\mathscr{H}_{F} \rightarrow L^{2}(\Omega)}=\left\|T_{F}\right\|_{L^{2}(\Omega) \rightarrow \mathscr{H}_{F}}
$$

for the respective operator-norms. While boundedness holds in "most" cases, it does not in general.

The assertion in (4.1.51) in Part (2) is a statement about the adjoint of an operator mapping between different Hilbert spaces; and it is a result of the following:

$$
\int_{\Omega} \overline{j(\xi)(x)} \varphi(x) d x=\left\langle\xi, T_{F} \varphi\right\rangle_{\mathscr{H}_{F}}\left(=\left\langle\xi, F_{\varphi}\right\rangle_{\mathscr{H}_{F}}\right)
$$

for all $\xi \in \mathscr{H}_{F}$, and $\varphi \in C_{c}(\Omega)$. But eq. (4.1.56), in turn, is immediate from (4.1.44) and the reproducing property in $\mathscr{H}_{F}$.

The assertion in Part 3 of the theorem follows from:

$$
\begin{aligned}
& \operatorname{Ker}(j)=\left(\operatorname{Ran}\left(j^{*}\right)\right)^{\perp} \\
& \underset{\text { by (2)) }}{=}\left(\operatorname{Ran}\left(T_{F}\right)\right)^{\perp}=0 \text {; }
\end{aligned}
$$

where we used that $\operatorname{Ran}\left(T_{F}\right)$ is dense in $\mathscr{H}_{F}$.

REMARK 4.1.23. The RHS in (4.1.51) is subtle because of boundary values for the function $\xi: \bar{\Omega} \rightarrow \mathbb{C}$ which represent some vector (also denoted $\xi$ ) in $\mathscr{H}_{F}$. We refer to Lemma 5.0.2 for specifics.

We showed that if $\varphi \in C_{c}(\Omega)$, then (4.1.56) holds; but if some $\varphi \in L^{2}(\Omega)$ is not in $C_{c}(\Omega)$, then we pick up a boundary term when computing $\left\langle\xi, T_{F} \varphi\right\rangle_{\mathscr{H}_{F}}$. Specifically, we show in section 2.6 (in the case of connected Lie groups) that there is a measure $\beta$ on the boundary $\partial \Omega=\bar{\Omega} \backslash \Omega$ such that

$$
\left\langle\xi, T_{F} \varphi\right\rangle_{\mathscr{H}_{F}}=\int_{\Omega} \overline{\xi(x)} \varphi(x) d x+\int_{\partial \Omega} \overline{\left.\xi\right|_{\partial \Omega}(\sigma)}\left(T_{F} \varphi\right)_{n}(\sigma) d \beta(\sigma)
$$

for all $\varphi \in L^{2}(\Omega)$.

In (4.1.57), on the RHS, we must make the following restrictions:

(1) $\bar{\Omega}$ is compact (and of course $\Omega$ is assumed open connected);

(2) $\partial \Omega$ is a differentiable manifold of dimension $=\operatorname{dim}(G)-1$.

(3) The function $T_{F} \varphi \in \mathscr{H}_{F} \subset C(\Omega)$ has a well-defined inward normal vector field; and $\left(T_{F} \varphi\right)_{n}$ denotes the corresponding normal derivative. 
It follows from Lemma 5.0.2 that the term $b_{\xi}(\sigma):=\left.\xi\right|_{\partial \Omega}(\sigma)$ on the RHS in (4.1.57) satisfies

$$
\left|b_{\xi}(\sigma)\right| \leq\|\xi\|_{\mathscr{H}_{F}}, \forall \sigma \in \partial \Omega .
$$

Corollary 4.1.24 (Renormalization). Let $G, \Omega, F, \mathscr{H}_{F}$, and $T_{F}$ be as in the statement of Theorem 4.1.22. Let $\left\{\xi_{n}\right\}_{n \in \mathbb{N}},\left\{\lambda_{n}\right\}_{n \in \mathbb{N}}$ be the spectral data for the Mercer operator $T_{F}$, i.e., $\lambda_{n}>0$,

$$
\begin{aligned}
& \sum_{n=1}^{\infty} \lambda_{n}=|\Omega|, \text { and }\left\{\xi_{n}\right\}_{n \in \mathbb{N}} \subset L^{2}(\Omega) \cap \mathscr{H}_{F}, \text { satisfying } \\
& T_{F} \xi_{n}=\lambda_{n} \xi_{n}, \int_{\Omega} \overline{\xi_{n}(x)} \xi_{m}(x) d x=\delta_{n, m}, n, m \in \mathbb{N},
\end{aligned}
$$

and

$$
F\left(x^{-1} y\right)=\sum_{n=1}^{\infty} \lambda_{n} \overline{\xi_{n}(x)} \xi_{n}(y), \forall x, y \in \Omega
$$

then

is an ONB in $\mathscr{H}_{F}$.

$$
\left\{\sqrt{\lambda_{n}} \xi_{n}(\cdot)\right\}_{n \in \mathbb{N}}
$$

Proof. This is immediate for the theorem. To stress the idea, we include the proof that $\left\|\sqrt{\lambda_{n}} \xi_{n}\right\|_{\mathscr{H}_{F}}=1, \forall n \in \mathbb{N}$.

Clearly,

$$
\left\|\sqrt{\lambda_{n}} \xi_{n}\right\|_{\mathscr{H}_{F}}^{2}=\lambda_{n}\left\langle\xi_{n}, \xi_{n}\right\rangle_{\mathscr{H}_{F}}=\left\langle\xi_{n}, T_{F} \xi_{n}\right\rangle_{\mathscr{H}_{F}} .
$$

But since $\xi_{n} \in L^{2}(\Omega) \cap \mathscr{H}_{F}$,

$$
\left\langle\xi_{n}, T_{F} \xi_{n}\right\rangle_{\mathscr{H}_{F}}=\int_{\Omega} \overline{\xi_{n}(x)} \xi_{n}(x) d x=\left\|\xi_{n}\right\|_{L^{2}(\Omega)}^{2}=1 \text { by }(4.1 .60),
$$

and the result follows.

Theorem 4.1.25. Let $G, \Omega, F, \mathscr{H}_{F},\left\{\xi_{n}\right\}$, and $\left\{\lambda_{n}\right\}$ be as specified in Corollary 4.1.24.

(1) Then $\mathscr{H}_{F} \subseteq L^{2}(\Omega)$, and there is a finite constant $C_{1}$ such that

$$
\int_{\Omega}|g(x)|^{2} d x \leq C_{1}\|g\|_{\mathscr{H}_{F}}
$$

holds for all $g \in \mathscr{H}_{F}$. Indeed, $C_{1}=\left\|\lambda_{1}\right\|_{\infty}$ will do.

(2) Let $\left\{\xi_{k}\right\},\left\{\lambda_{k}\right\}$ be as above, let $N \in \mathbb{N}$, set

$$
\mathscr{H}_{F}(N)=\operatorname{span}\left\{\xi_{k} \mid k=1,2,3, \ldots, N\right\} ;
$$

and let $Q_{N}$ be the $\mathscr{H}_{F}$-orthogonal projection onto $\mathscr{H}_{F}(N)$; and let $P_{N}$ be the $L^{2}(\Omega)$-orthogonal projection onto $\operatorname{span}_{1 \leq k \leq N}\left\{\xi_{k}\right\}$; then

$$
Q_{N} \geq\left(\frac{1}{\lambda_{1}}\right) P_{N}
$$

where $\leq$ in (4.1.65) is the order of Hermitian operators, and where $\lambda_{1}$ is the largest eigenvalue. 
Proof. Part (1). Pick $\lambda_{n}, \xi_{n}$ as in (4.1.60)-(4.1.62). We saw that $\mathscr{H}_{F} \subset$ $L^{2}(\Omega)$; recall $\bar{\Omega}$ is compact. Hence

$$
\begin{aligned}
\|g\|_{L^{2}(\Omega)}^{2} & =\sum_{n=1}^{\infty}\left|\left\langle\xi_{n}, g\right\rangle_{2}\right|^{2} \\
& =\sum_{n=1}^{\infty}\left|\left\langle T_{F} \xi_{n}, g\right\rangle_{\mathscr{H}_{F}}\right|^{2} \\
& =\sum_{n=1}^{\infty}\left|\left\langle\lambda_{n} \xi_{n}, g\right\rangle_{\mathscr{H}_{F}}\right|^{2} \\
& =\sum_{n=1}^{\infty} \lambda_{n}\left|\left\langle\sqrt{\lambda_{n}} \xi_{n}, g\right\rangle_{\mathscr{H}_{F}}\right|^{2} \\
& \leq\left(\sup _{n \in \mathbb{N}}\left\{\lambda_{n}\right\}\right) \sum_{n=1}^{\infty}\left|\left\langle\sqrt{\lambda_{n}} \xi_{n}, g\right\rangle_{\mathscr{H}_{F}}\right|^{2} \\
& =\left(\sup _{n \in \mathbb{N}}\left\{\lambda_{n}\right\}\right)\|g\|_{\mathscr{H}_{F}}^{2}, \text { by }(4.1 .62) \text { and Parseval. }
\end{aligned}
$$

This proves (1) with $C_{1}=\sup _{n \in \mathbb{N}}\left\{\lambda_{n}\right\}$.

Part (2). Let $f \in L^{2}(\Omega) \cap \mathscr{H}_{F}$. Arrange the eigenvalues $\left(\lambda_{n}\right)$ s.t.

$$
\lambda_{1} \geq \lambda_{2} \geq \lambda_{3} \cdots>0 .
$$

Then

$$
\begin{aligned}
\left\langle f, Q_{N} f\right\rangle_{\mathscr{H}_{F}}=\left\|Q_{N} f\right\|_{\mathscr{H}_{F}}^{2} & \stackrel{(4.1 .62)}{=} \quad \sum_{n=1}^{N}\left|\left\langle\sqrt{\lambda_{n}} \xi_{n}, f\right\rangle_{\mathscr{H}_{F}}\right|^{2} \\
& =\sum_{n=1}^{N} \frac{1}{\lambda_{n}}\left|\left\langle\lambda_{n} \xi_{n}, f\right\rangle_{\mathscr{H}_{F}}\right|^{2} \\
& =\sum_{n=1}^{N} \frac{1}{\lambda_{n}}\left|\left\langle T_{F} \xi_{n}, f\right\rangle_{\mathscr{H}_{F}}\right|^{2} \\
& =\sum_{n=1}^{N} \frac{1}{\lambda_{n}}\left|\left\langle\xi_{n}, f\right\rangle_{L^{2}(\Omega)}\right|^{2} \\
& \stackrel{(4.1 .66)}{\geq} \frac{1}{\lambda_{1}} \sum_{n=1}^{N}\left|\left\langle\xi_{n}, f\right\rangle_{L^{2}(\Omega)}\right|^{2} \\
& \stackrel{\text { (Parseval) }}{=} \frac{1}{\lambda_{1}}\left\|P_{N} f\right\|_{L^{2}(\Omega)}^{2}=\frac{1}{\lambda_{1}}\left\langle f, P_{N} f\right\rangle_{L^{2}(\Omega)} ;
\end{aligned}
$$

and so the a priori estimate (2) holds.

REMARK 4.1.26. The estimate (4.1.63) in Theorem 4.1.25 is related to, but different from a classical Poincaré-inequality [Maz11]. The latter a priori is as follows: 
Let $\Omega \subset \mathbb{R}^{k}$ satisfying in B1-B4 in Remark 4.1.17, and let $|\Omega|_{k}$ denote the $k$-dimensional Lebesgue measure of $\Omega$, i.e.,

$$
|\Omega|_{k}=\int_{\mathbb{R}^{k}} \chi_{\Omega}(x) \underbrace{d x_{1} \cdots d x_{k}}_{d x} ;
$$

let $\nabla=\left(\frac{\partial}{\partial x_{1}}, \ldots, \frac{\partial}{\partial x_{k}}\right)$ be the gradient, and

$$
\|\nabla f\|_{L^{2}(\Omega)}^{2}:=\sum_{i=1}^{k} \int_{\Omega}\left|\frac{\partial f}{\partial x_{i}}\right|^{2} d x .
$$

Finally, let $\lambda_{1}(N)=$ the finite eigenvalue for the Neumann problem on $\Omega(\mathrm{NBP} \Omega)$. Then,

$$
\left\|f-\frac{1}{|\Omega|_{k}} \int_{\Omega} f d x\right\|_{L^{2}(\Omega)}^{2} \leq \frac{1}{\lambda_{1}(N)}\|\nabla f\|_{L^{2}(\Omega)}^{2}
$$

holds for all $f \in L^{2}(\Omega)$ such that $\frac{\partial f}{\partial x_{i}} \in L^{2}(\Omega), 1 \leq i \leq k$.

Definition 4.1.27. A system of functions $\left\{f_{n}\right\}_{n \in \mathbb{Z}} \subset \mathscr{H}_{F}$ is said to be a Bessel frame [CM13] if there is a finite constant $A$ such that

$$
\sum_{n \in \mathbb{Z}}\left|\left\langle f_{n}, \xi\right\rangle_{\mathscr{H}_{F}}\right|^{2} \leq A\|\xi\|_{\mathscr{H}_{F}}^{2}, \forall \xi \in \mathscr{H}_{F} .
$$

TheOrem 4.1.28. Let $F:(-1,1) \rightarrow \mathbb{C}$ be continuous and p.d., and let $\left\{f_{n}\right\}_{n \in \mathbb{Z}} \subset$ $\mathscr{H}_{F}$ be the system (4.1.37) obtained by Shannon sampling in Fourier-domain; then $\left\{f_{n}\right\}$ is a Bessel frame, where we may take $A=\lambda_{1}$ as frame bound in (4.1.69). $\left(\lambda_{1}=\right.$ the largest eigenvalue of the Mercer operator $\left.T_{F}.\right)$

Proof. Let $\xi \in \mathscr{H}_{F}$, then

$$
\begin{array}{rll}
\sum_{n \in \mathbb{Z}}\left|\left\langle f_{n}, \xi\right\rangle_{\mathscr{H}_{F}}\right|^{2} & = & \sum_{n \in \mathbb{Z}}\left|\left\langle T e_{n}, \xi\right\rangle_{\mathscr{H}_{F}}\right|^{2} \\
& \underset{\text { Cor. }}{\stackrel{(4.1 .24)}{=}} & \sum_{n \in \mathbb{Z}}\left|\left\langle e_{n}, \xi\right\rangle_{L^{2}(0,1)}\right|^{2} \\
\underset{\text { (Parseval) }}{=} & \|\xi\|_{L^{2}(0,1)}^{2} \\
& \lambda_{1}\|\xi\|_{\mathscr{H}_{F}}^{2}
\end{array}
$$

which is the desired conclusion.

At the start of the estimate above we used the Fourier basis $e_{n}(x)=e^{i 2 \pi n x}$, $n \in \mathbb{Z}$, an ONB in $L^{2}(0,1)$; and the fact that $f_{n}=T_{F}\left(e_{n}\right), n \in \mathbb{Z}$; see the details in the proof of Theorem 4.1.13.

Corollary 4.1.29. For every $f \in L^{2}(\Omega) \cap \mathscr{H}_{F}$, and every $x \in \bar{\Omega}$ (including boundary points), we have the following estimate:

$$
|f(x)| \leq\|f\|_{\mathscr{H}_{F}}
$$

(Note that this is independent of $x$ and of $f$.)

Proof. The estimate in (4.1.70) follows from Lemma 5.0.2; and (4.1.65) in part (2) of Theorem 4.1.25. 
Corollary 4.1.30. Let $\left\{\xi_{n}\right\},\left\{\lambda_{n}\right\}, T_{F}, \mathscr{H}_{F}$ and $L^{2}(\Omega)$ be as above; and assume $\lambda_{1} \geq \lambda_{2} \geq \cdots$; then we have the following details for operator norms

$$
\begin{aligned}
\left\|\left.T_{F}^{-1}\right|_{\operatorname{span}\left\{\xi_{k}: k=1, \ldots, N\right\}}\right\|_{\mathscr{H}_{F} \rightarrow \mathscr{H}_{F}} & =\left\|\left.T_{F}^{-1}\right|_{\operatorname{span}\left\{\xi_{k}: k=1, \ldots, N\right\}}\right\|_{L^{2}(\Omega) \rightarrow L^{2}(\Omega)} \\
& =\frac{1}{\lambda_{N}} \longrightarrow \infty \text {, as } N \rightarrow \infty
\end{aligned}
$$

Since we shall not have occasion to use this more general version of the Merceroperators we omit details below, and restrict attention to the case of finite interval in $\mathbb{R}$.

Remark 4.1.31. Some of the conclusions in Theorem 4.1.22 hold even if conditions B1-B4 are relaxed. But condition B3 ensures that $L^{2}(\Omega)$ has a realization as a subspace of $\mathscr{H}_{F}$; see eq. (4.1.50). By going to unbounded sets $\Omega$ we give up this.

Even if $\Omega \subset G$ is unbounded, then the operator $T_{F}$ in (4.1.44) is still welldefined; and it may be considered as a possibly unbounded linear operator as follow:

$$
L^{2}(\Omega) \stackrel{T_{F}}{\longrightarrow} \mathscr{H}_{F}
$$

with dense domain $C_{c}(\Omega)$ in $L^{2}(\Omega)$. (If $G$ is a Lie group, we may take $C_{c}^{\infty}(\Omega)$ as dense domain for $T_{F}$ in (4.1.72).)

Lemma 4.1.32. Let $(\Omega, F)$ be as above, but now assume only conditions B1 and B2 for the subset $\Omega \subset G$.

Then the operator $T_{F}$ in (4.1.72) is a closable operator from $L^{2}(\Omega)$ into $\mathscr{H}_{F}$; i.e., the closure of the graph of $T_{F}$, as a subspace in $L^{2}(\Omega) \times \mathscr{H}_{F}$, is the graph of a (closed) operator $\overline{T_{F}}$ from $L^{2}(\Omega)$ into $\mathscr{H}_{F}$; still with dom $\left(\overline{T_{F}}\right)$ dense in $L^{2}(\Omega)$.

Proof. Using a standard lemma on unbounded operators, see [Rud73, ch.13], we need only show that the following implication holds:

Given $\left\{f_{n}\right\} \subset C_{c}(\Omega)\left(\subset L^{2}(\Omega)\right)$, suppose $\exists \xi \in \mathscr{H}_{F}$; and suppose the following two limits holds:

$$
\begin{aligned}
& \lim _{n \rightarrow \infty}\left\|f_{n}\right\|_{L^{2}(\Omega)}=0, \text { and } \\
& \lim _{n \rightarrow \infty}\left\|\xi-T_{F}\left(f_{n}\right)\right\|_{\mathscr{H}_{F}}=0 .
\end{aligned}
$$

Then, it follows that $\xi=0$ in $\mathscr{H}_{F}$.

Now assume $\left\{f_{n}\right\}$ and $\xi$ satisfying (4.1.73)-(4.1.74); the by the reproducing property in $\mathscr{H}_{F}$, we have

$$
\left\langle\xi, T_{F} f_{n}\right\rangle_{\mathscr{H}_{F}}=\int_{\Omega} \overline{\xi(x)} f_{n}(x) d x
$$

Using (4.1.74), we get

$$
\lim _{n \rightarrow \infty}(\mathrm{LHS})_{(4.1 .75)}=\langle\xi, \xi\rangle_{\mathscr{H}_{F}}=\|\xi\|_{\mathscr{H}_{F}}^{2} ;
$$

and using (4.1.73), we get

$$
\lim _{n \rightarrow \infty}(\mathrm{RHS})_{(4.1 .75)}=0 .
$$

The domination here is justified by (4.1.74). Indeed, if (4.1.74) holds, $\exists n_{0}$ s.t.

$$
\left\|\xi-T_{F}\left(f_{n}\right)\right\|_{\mathscr{H}_{F}} \leq 1, \forall n \geq n_{0},
$$


and therefore,

$$
\sup _{n \in \mathbb{N}}\left\|T_{F}\left(f_{n}\right)\right\|_{\mathscr{H}_{F}} \leq \max _{n \leq n_{0}}\left(1+\left\|T_{F}\left(f_{n}\right)\right\|_{\mathscr{H}_{F}}\right)<\infty .
$$

The desired conclusion follows; we get $\xi=0$ in $\mathscr{H}_{F}$.

REMARK 4.1.33. The conclusion in Lemma 4.1.32 is the assertion that the closure of the graph of $T_{F}$ is again the graph of a closed operator, called the closure. Hence the importance of "closability." Once we have existence of the closure of the operator $T_{F}$, as a closed operator, we will denote this closed operator also by the same $T_{F}$. This helps reduce the clutter in operator symbols to follow. From now on, $T_{F}$ will be understood to be the closed operator obtained in Lemma 4.1.32.

REMARK 4.1.34. If in Lemma 4.1.32, for $(F, \Omega)$ the set $\Omega$ also satisfies B3-B4, then the operator $T_{F}$ in (4.1.72) is in fact bounded; but in general it is not; see the example below with $G=\mathbb{R}$, and $\Omega=\mathbb{R}_{+}$.

Corollary 4.1.35. Let $\Omega, G$ and $F$ be as in Lemma 4.1.32, i.e., with $\Omega$ possibly unbounded, and let $T_{F}$ denote the closed operator obtained from (4.1.72), and the conclusion in the lemma. Then we get the following two conclusions:

(1) $T_{F}^{*} T_{F}$ is selfadjoint with dense domain in $L^{2}(\Omega)$, and

(2) $T_{F} T_{F}^{*}$ is selfadjoint with dense domain in $\mathscr{H}_{F}$.

Proof. This is an application of the fundamental theorem for closed operators; see [Rud73, Theorem 13.13].

REMARK 4.1.36. The significance of the conclusions (1)-(2) in the corollary is that we may apply the spectral theorem to the respective selfadjoint operators in order to get that $\left(T_{F}^{*} T_{F}\right)^{1 / 2}$ is a well-defined selfadjoint operator in $L^{2}(\Omega)$; and that $\left(T_{F} T_{F}^{*}\right)^{1 / 2}$ well-defined and selfadjoint in $\mathscr{H}_{F}$.

Moreover, by the polar decomposition applied to $T_{F}$ (see [Rud73, ch. 13]), we conclude that:

$$
\operatorname{spec}\left(T_{F}^{*} T_{F}\right) \backslash\{0\}=\operatorname{spec}\left(T_{F} T_{F}^{*}\right) \backslash\{0\} .
$$

Theorem 4.1.37. Assume $F, G, \Omega$, and $T_{F}$, are as above, where $T_{F}$ denotes the closed operator $L^{2}(\Omega) \stackrel{T}{\longrightarrow} \mathscr{H}_{F}$. We are assuming that $G$ is a Lie group, $\Omega$ satisfies B1-B2. Let $X$ be a vector in the Lie algebra of $G, X \in L a(G)$, and define $D_{X}^{(F)}$ as a skew-Hermitian operator in $\mathscr{H}_{F}$ as follows:

$$
\begin{aligned}
\operatorname{dom}\left(D_{X}^{(F)}\right) & =\left\{T_{F} \varphi \mid \varphi \in C_{c}^{\infty}(\Omega)\right\}, \text { and } \\
D_{X}^{(F)}\left(F_{\varphi}\right) & =F_{\widetilde{X} \varphi}
\end{aligned}
$$

where

$$
(\widetilde{X} \varphi)(g)=\lim _{t \rightarrow 0} \frac{1}{t}(\varphi(\exp (-t X) g)-\varphi(g))
$$

for all $\varphi \in C_{c}^{\infty}(\Omega)$, and all $g \in \Omega$.

Then $\widetilde{X}$ defines a skew-Hermitian operator in $L^{2}(\Omega)$ with dense domain $C_{c}^{\infty}(\Omega)$. (It is closable, and we shall denote its closure also by $\widetilde{X}$.)

We get

$$
D_{X}^{(F)} T_{F}=T_{F} \widetilde{X}
$$


on the domain of $\tilde{X}$; or equivalently

$$
D_{X}^{(F)}=T_{F} \widetilde{X} T_{F}^{-1} .
$$

Proof. By definition, for all $\varphi \in C_{c}^{\infty}(\Omega)$, we have

$$
\left(D_{X}^{(F)} T_{F}\right)(\varphi)=D_{X}^{(F)} F_{\varphi}=F_{\tilde{X} \varphi}=\left(T_{F} \tilde{X}\right)(\varphi) .
$$

Since $\left\{F_{\varphi} \mid \varphi \in C_{c}^{\infty}(\Omega)\right\}$ is a core-domain, (4.1.80) follows. Then the conclusions in the theorem follow from a direct application of Lemma 4.1.32, and Corollary 4.1.35; see also Remark 4.1.36.

Corollary 4.1.38. For the respective adjoint operators in (4.1.81), we have

$$
\left(D_{X}^{(F)}\right)^{*}=T_{F}^{*-1} \tilde{X}^{*} T_{F}^{*} .
$$

PROOF. The formula (4.1.82) in the corollary results from applying the adjoint operation to both sides of eq (4.1.81), and keeping track of the domains of the respective operators in the product on the RHS in eq (4.1.81). Only after checking domains of the three respective operators, occurring as factors in the product on the RHS in eq (4.1.81), may we then use the algebraic rules for adjoint of a product of operators. In this instance, we conclude that adjoint of the product on the RHS in eq (4.1.81) is the product of the adjoint of the factors, but now composed in the reverse order; so product from left to right, becomes product of the adjoints from right to left; hence the result on the RHS in eq (4.1.82).

Now the fact that the domain issues work out follows from application of Corollary 4.1.35, Remark 4.1.36, and Theorem 4.1.37; see especially eqs (4.1.77), and (4.1.78). The rules for adjoint of a product of operators, where some factors are unbounded are subtle, and we refer to [Rud73, chapter 13] and [DS88, Chapter 11-12]. Care must be exercised when the unbounded operators in the product map between different Hilbert spaces. The fact that our operator $T_{F}$ is closed as a linear operator from $L^{2}(\Omega)$ into $\mathscr{H}_{F}$ is crucial in this connection; see Lemma 4.1.32.

Corollary 4.1.39. Let $G, \Omega, F$, and $T_{F}$ be as above; then the RKHS $\mathscr{H}_{F}$ consists precisely of the continuous functions $\xi$ on $\Omega$ such that $\xi \in \operatorname{dom}\left(\left(T_{F}^{*} T_{F}\right)^{-1 / 4}\right)$, and then

$$
\|\xi\|_{\mathscr{H}_{F}}=\left\|\left(T_{F}^{*} T_{F}\right)^{-1 / 4} \xi\right\|_{L^{2}(\Omega)} .
$$

Proof. An immediate application of Corollary 4.1.35; and the polar decomposition, applied to the closed operator $T_{F}$ from Lemma 4.1.32.

ExAmple 4.1.40 (Application). Let $G=\mathbb{R}, \Omega=\mathbb{R}_{+}=(0, \infty)$; so that $\Omega-\Omega=$ $\mathbb{R}$; let $F(x)=e^{-|x|}, \forall x \in \mathbb{R}$, and let $D^{(F)}$ be the skew-Hermitian operator from Corollary 4.1.38. Then $D^{(F)}$ has deficiency indices $(1,0)$ in $\mathscr{H}_{F}$.

Proof. From Corollary 4.1.35, we conclude that $\mathscr{H}_{F}$ consists of all continuous functions $\xi$ on $\mathbb{R}_{+}(=\Omega)$ such that $\xi$ and $\xi^{\prime}=\frac{d \xi}{d x}$ are in $L^{2}\left(\mathbb{R}_{+}\right)$; and then

$$
\|\xi\|_{\mathscr{H}_{F}}^{2}=\frac{1}{2}\left(\int_{0}^{\infty}|\xi(x)|^{2} d x+\int_{0}^{\infty}\left|\xi^{\prime}(x)\right|^{2} d x\right)+\int_{0}^{1} \overline{\xi_{n}} \xi d \beta
$$

where $\xi_{n}$ denote its inward normal derivative, and $d \beta$ is the corresponding boundary measure. Indeed, $d \beta=-\frac{1}{2} \delta_{0}$, with $\delta_{0}:=\delta(\cdot-0)=$ Dirac mass at $x=0$. See sections 4.3.2-4.3.3 for details. 
We now apply Corollary 4.1 .38 to the operator $D_{0}=\frac{d}{d x}$ in $L^{2}\left(\mathbb{R}_{+}\right)$with $\operatorname{dom}\left(D_{0}\right)=C_{c}^{\infty}\left(\mathbb{R}_{+}\right)$. It is well known that $D_{0}$ has deficiency indices $(1,0)$; and the + deficiency space is spanned by $\xi_{+}(x):=e^{-x} \in L^{2}\left(\mathbb{R}_{+}\right)$, i.e., $x>0$.

Hence, using (4.1.82), we only need to show that $\xi_{+} \in \mathscr{H}_{F}$; but this is immediate from (4.1.83); in fact

$$
\left\|\xi_{+}\right\|_{\mathscr{H}_{F}}^{2}=1
$$

Setting $\xi_{-}(x):=e^{x}$, the same argument shows that $\operatorname{RHS}_{(4.1 .83)}=\infty$, so the index conclusion $(1,0)$ follows.

We now return to the case for $G=\mathbb{R}$, and $F$ is fixed continuous positive definite function on some finite interval $(-a, a)$, i.e., the case where $\Omega=(0, a)$.

COROLlaRY 4.1.41. If $G=\mathbb{R}$ and if $\Omega=(0, a)$ is a bounded interval, $a<\infty$, then the operator $D^{(F)}$ has equal indices for all given $F:(-a, a) \rightarrow \mathbb{C}$ which is p.d. and continuous.

Proof. We showed in Theorem 4.1.22, and Corollary 4.1.24 that if $\Omega=(0, a)$ is bounded, then $T_{F}: L^{2}(0, a) \rightarrow \mathscr{H}_{F}$ is bounded. By Corollary 4.1.35, we get that $T_{F}^{-1}: \mathscr{H}_{F} \rightarrow L^{2}(0, a)$ is closed. Moreover, as an operator in $L^{2}(0, a), T_{F}$ is positive and selfadjoint.

Since

$$
D_{0}=\left.\frac{d}{d x}\right|_{C_{c}^{\infty}(0, a)}
$$

has indices $(1,1)$ in $L^{2}(0, a)$, it follows from (4.1.82) applied to (4.1.84) that $D^{(F)}$, as a skew-Hermitian operator in $\mathscr{H}_{F}$, must have indices $(0,0)$ or $(1,1)$.

To finish the proof, use that a skew Hermitian operator with indices $(1,0)$ must generate a semigroup of isometries; one that is non-unitary. If such an isometry semigroup were generated by the particular skew Hermitian operator $D^{(F)}$ then this would be inconsistent with Corollary 4.1.39; see especially the formula for the norm in $\mathscr{H}_{F}$.

To simplify notation, we now assume that the endpoint $a$ in equation (4.1.1) is $a=1$.

Proposition 4.1.42. Let $F$ be p.d. continuos on $I=(-1,1) \subset \mathbb{R}$. Assume $\mu \in \operatorname{Ext}(F)$, and $\mu \ll d \lambda$, i.e., $\exists M \in L^{1}(\mathbb{R})$ s.t.

$$
d \mu(\lambda)=M(\lambda) d \lambda \text {, where } d \lambda=\text { Lebesgue measure on } \mathbb{R} .
$$

Set $\mathscr{L}=(2 \pi \mathbb{Z})($ period lattice $)$, and

$$
\widehat{\varphi_{I}}(\xi)=\int_{0}^{1} e^{-i \xi y} \varphi(y) d y, \forall \varphi \in C_{c}(0,1)
$$

then the Mercer operator is as follows:

$$
\left(T_{F} \varphi\right)(x)=\sum_{l \in \mathscr{L}} M(l) \widehat{\varphi_{I}(l)} e^{i l x} .
$$

Proof. Let $x \in(0,1)$, then

$$
\operatorname{RHS}_{(4.1 .87)}(x) \quad=\quad \sum_{l \in \mathscr{L}} M(l)\left(\int_{0}^{1} e^{-i l y} \varphi(y) d y\right) e^{i l x}
$$




$$
\begin{aligned}
\text { (Fubini) } & \int_{0}^{1} \varphi(y) \underbrace{\left(\sum_{l \in \mathscr{L}} M(l) e^{i l(x-y)}\right)}_{\text {Poisson summation }} d y \\
& =\quad \int_{0}^{1} \varphi(y) F(x-y) d y \\
& =\quad\left(T_{F} \varphi\right)(x) \\
& =\quad \operatorname{LHS}_{(4.1 .87)},
\end{aligned}
$$

where we use that $F=\left.\widehat{d \mu}\right|_{(-1,1)}$, and (4.1.85).

ExAmple 4.1.43. Application to Table on page 52: $\mathscr{L}=2 \pi \mathbb{Z}$.

Proof. Application of Proposition 4.1.42. See Table 4.1.2 below.

\begin{tabular}{|c|c|c|}
\hline p.d. Function & $\left(T_{F} \varphi\right)(x)$ & $M(\lambda), \lambda \in \mathbb{R}$ \\
\hline$F_{1}$ & $\frac{1}{2} \sum_{l \in \mathscr{L}} e^{-|l|} \widehat{\varphi}_{I}(l) e^{i l x}$ & $\frac{1}{2} e^{-|l|}$ \\
\hline$F_{3}$ & $\sum_{l \in \mathscr{L}} \frac{1}{\pi\left(1+l^{2}\right)} \widehat{\varphi}_{I}(l) e^{i l x}$ & $\frac{1}{\pi\left(1+l^{2}\right)}$ \\
\hline$F_{5}$ & $\sum_{l \in \mathscr{L}} \frac{1}{\sqrt{2 \pi}} e^{-l^{2} / 2} \widehat{\varphi}_{I}(l) e^{i l x}$ & $\frac{1}{\sqrt{2 \pi}} e^{-l^{2} / 2}$ \\
\hline
\end{tabular}

Table 4.1.2. Application of Proposition 4.1.42 to Table 3.1.1.

Corollary 4.1.44. Let $F:(-1,1) \rightarrow \mathbb{C}$ be a continuous positive definite function on the interval $(-1,1)$, and assume:

(i) $F(0)=1$

(ii) $\exists \mu \in \operatorname{Ext}_{1}(F)$ s.t. $\mu \ll d \lambda$, i.e., $\exists M \in L^{1}(\mathbb{R})$ s.t. $d \mu(\lambda)=M(\lambda) d \lambda$ on $\mathbb{R}$.

Now consider the Mercer operator

$$
\left(T_{F} \varphi\right)(x)=\int_{0}^{1} \varphi(y) F(x-y) d y, \varphi \in L^{2}(0,1), x \in(0,1) .
$$

Then the following two conditions (bd-1) and (bd-2) are equivalent, where

$$
\begin{array}{ll} 
& \mathscr{L}=(2 \pi \mathbb{Z})=\widehat{\mathbb{T}}, \text { and } \\
(b d-1) & b_{M}:=\sup _{\lambda \in[0,1]} \sum_{l \in \mathscr{L}} M(\lambda+l)<\infty, \text { and } \\
\mathbb{1} & \\
(b d-2) & T_{F}\left(L^{2}(0,1)\right) \subseteq \mathscr{H}_{F} .
\end{array}
$$

If (bd-1) ( $\Leftrightarrow(b d-2))$ holds, then, for the corresponding operator-norm, we then have

$$
\left\|T_{F}\right\|_{L^{2}(0,1) \rightarrow \mathscr{H}_{F}}=\sqrt{b_{M}} \text { in }(b d-1) .
$$


REMARK 4.1.45. Condition (bd-1) is automatically satisfied in all interesting cases (at least from the point of view of our present Memoir.)

Proof. The key step in the proof of " $\Longleftrightarrow$ " was the Parseval duality,

$$
\int_{0}^{1}|f(x)|^{2} d x=\sum_{l \in \mathscr{L}}\left|\widehat{f}_{I}(l)\right|^{2} \text {, where }
$$

$[0,1) \simeq \mathbb{T}=\mathbb{R} / \mathbb{Z}, \widehat{\mathbb{T}} \simeq \mathscr{L}$

Let $F, T_{F}$, and $M$ be as in the statement of the corollary. Then for $\varphi \in C_{c}(0,1)$, we compute the $\mathscr{H}_{F}$-norm of

$$
T_{F}(\varphi)=F_{\varphi}
$$

with the use of (4.1.88), and Proposition 4.1.42.

We return to

$$
\widehat{\varphi_{I}}(l)=\int_{0}^{1} e^{-i l y} \varphi(y) d y, l \in \mathscr{L}
$$

and we now compute $\left(\widehat{T} F \varphi_{I}(l), l \in \mathscr{L}\right.$; starting with $T_{F} \varphi$ from (4.1.88). The result is

$$
\widehat{\left(T_{F} \varphi\right)_{I}}(l)=M(l) \widehat{\varphi_{I}}(l), \forall l \in \mathscr{L}(=2 \pi \mathbb{Z} .)
$$

And further, using chapter 6 , we have:

$$
\begin{aligned}
& \left\|F_{\varphi}\right\|_{\mathscr{H}_{F}}^{2} \quad=\quad\left\|T_{F} \varphi\right\|_{\mathscr{H}_{F}}^{2} \\
& \underset{(\text { Cor. }(2.4 .9))}{=} \int_{\mathbb{R}}|\widehat{\varphi}(\lambda)|^{2} M(\lambda) d \lambda \\
& =\quad \int_{0}^{1} \sum_{l \in \mathscr{L}}|\widehat{\varphi}(\lambda+l)|^{2} M(\lambda+l) d \lambda \\
& \underset{(4.1 .93)}{\leq} \quad\left(\sum_{l \in \mathscr{L}}|\widehat{\varphi}(l)|^{2}\right) \sup _{\lambda \in[0,1)} \sum_{l \in \mathscr{L}} M(\lambda+l) \\
& \underset{(4.1 .91)}{=} \quad\left(\int_{0}^{1}|\varphi(x)|^{2} d x\right) \cdot b_{M}=\|\varphi\|_{L^{2}(0,1)}^{2} \cdot b_{M} . \\
& \text { and (bd-1) }
\end{aligned}
$$

Hence, if $b_{M}<\infty$, (bd-2) holds, with

$$
\left\|T_{F}\right\|_{L^{2}(0,1) \rightarrow \mathscr{H}_{F}} \leq \sqrt{b_{M}} .
$$

Using standard Fourier duality, one finally sees that " $\leq$ " in (4.1.94) is in fact "=".

REMARK 4.1.46. A necessary condition for boundedness of $T_{F}: L^{2}(0,1) \rightarrow$ $\mathscr{H}_{F}$, is $M \in L^{\infty}(\mathbb{R})$ when the function $M(\cdot)$ is as specified in (ii) of the corollary.

Proof. Let $\varphi \in C_{c}(0,1)$, then

$$
\begin{aligned}
\left\|T_{F} \varphi\right\|_{\mathscr{H}_{F}}^{2}=\left\|F_{\varphi}\right\|_{\mathscr{H}_{F}}^{2} & =\int_{\mathbb{R}}|\widehat{\varphi}(\lambda)|^{2} M(\lambda) d \lambda \\
& \leq\|M\|_{\infty} \cdot \int_{\mathbb{R}}|\widehat{\varphi}(\lambda)|^{2} d \lambda
\end{aligned}
$$




$$
\begin{aligned}
& =\|M\|_{\infty} \cdot \int_{\mathbb{R}}|\varphi(x)|^{2} d \lambda \quad \text { (Parseval) } \\
& =\|M\|_{\infty}\|\varphi\|_{L^{2}(0,1)}^{2} .
\end{aligned}
$$

Theorem 4.1.47. Let $F$ be as in Proposition 4.1.42, and $\mathscr{H}_{F}$ the corresponding RKHS. Define the skew-Hermitian operator $D^{(F)}\left(F_{\varphi}\right)=\frac{1}{i} F_{\varphi^{\prime}}$ on

$$
\operatorname{dom}\left(D^{(F)}\right)=\left\{F_{\varphi} \mid \varphi \in C_{c}^{\infty}(0,1)\right\} \subset \mathscr{H}_{F}
$$

as before. Let $A \supset D^{(F)}$ be a selfadjoint extension of $D^{(F)}$, i.e.,

$$
D^{(F)} \subset A \subset\left(D^{(F)}\right)^{*}, A=A^{*} .
$$

Let $P=P_{A}$ be the projection valued measure (PVM) of $A$, and

$$
U_{t}^{(A)}=e^{t A}=\int_{\mathbb{R}} e^{i t \lambda} P_{A}(d \lambda), t \in \mathbb{R}
$$

be the one-parameter unitary group; and for all $f$ measurable on $\mathbb{R}$, set (the Spectral Theorem applied to A)

$$
f(A)=\int_{\mathbb{R}} f(\lambda) P_{A}(d \lambda) ;
$$

then we get the following

$$
\left(T_{F} \varphi\right)(x)=\left(M \widehat{\varphi_{I}}\right)(A) U_{x}^{(A)}=U_{x}^{(A)}\left(M \widehat{\varphi_{I}}\right)(A)
$$

for the Mercer operator $\left(T_{F} \varphi\right)(x)=\int_{0}^{1} F(x-y) \varphi(y), \varphi \in L^{2}(0,1)$.

Proof. Using (4.1.96), we get

$$
\begin{aligned}
\widehat{\varphi_{I}}(A) U_{x}^{(A)} & =\int_{\mathbb{R}} \int_{0}^{1} \varphi(y) e^{-i \lambda y} P_{A}(d \lambda) U^{A}(x) \\
& =\int_{(\text {Fubini) }}^{1} \varphi(y)\left(\int_{\mathbb{R}} e^{i \lambda(x-y)} P_{A}(d \lambda)\right) d y \\
& =\int_{0}^{1} U^{A}(x-y) \varphi(y) d y \\
& =\quad U_{x}^{(A)} U^{(A)}(\varphi), \text { where } \\
U^{(A)}(\varphi) & =\int_{0}^{1} \varphi(y) U^{(A)}(-y) d y
\end{aligned}
$$

all operators in the RKHS $\mathscr{H}_{F}$.

We have a selfadjoint extension $A$ corresponding to $\mu=\mu^{(A)} \in \operatorname{Ext}(F)$, and a cyclic vector $v_{0}$ :

$$
\begin{aligned}
F^{(A)}(t) & =\left\langle v_{0}, U^{(A)}(t) v_{0}\right\rangle \\
& =\int_{\mathbb{R}} e^{i \lambda t} d \mu_{A}(\lambda), \text { where } d \mu_{A}(\lambda)=\left\|P_{A}(d \lambda) v_{0}\right\|^{2},
\end{aligned}
$$

and from (4.1.99):

$$
\left(T_{F} \varphi\right)(x)=\left(F_{\varphi}^{(A)}\right)(x), \forall \varphi \in C_{c}(0,1), \text { and } \forall x \in(0,1) .
$$




\subsection{Positive Definite Functions, Green's Functions, and Boundary}

In this section, we consider a correspondence and interplay between a class of boundary value problems on the one hand, and spectral theoretic properties of extension operators on the other.

Fix a bounded domain $\Omega \subset \mathbb{R}^{n}$, open and connected. Let $F: \Omega-\Omega \rightarrow \mathbb{C}$ be a continuous positive definite (p.d.) function. We consider a special case when $F$ occurs as the Green's function of certain linear operator.

Lemma 4.2.1. Let $\mathscr{D}$ be a Hilbert space, a Fréchet space or an LF-space (see [Trè06]), such that $\mathscr{D} \underset{j}{\hookrightarrow} L^{2}(\Omega)$; and such that the inclusion mapping $j$ is continuous relative to the respective topologies on $\mathscr{D}$, and on $L^{2}(\Omega)$. Let $\mathscr{D}^{*}:=$ the dual of $\mathscr{D}$ when $\mathscr{D}$ is given its Fréchet (LF, or Hilbert) topology; then there is a natural "inclusion" mapping $j^{*}$ from $L^{2}(\Omega)$ to $\mathscr{D}^{*}$, i.e., we get

$$
\mathscr{D} \underset{j}{\hookrightarrow} L^{2}(\Omega) \underset{j^{*}}{\hookrightarrow} \mathscr{D}^{*} .
$$

Proof. It is immediate from the assumptions, and the fact that $L^{2}(\Omega)$ is its own dual. See also [Trè06].

REMARK 4.2.2. In the following we shall use lemma 4.2.1 in two cases:

(1) Let $A$ be a selfadjoint operator (unbounded in the non-trivial cases) acting in $L^{2}(\Omega)$; and with dense domain. For $\mathscr{D}=\mathscr{D}_{A}$, we may choose the domain of $A$ with its graph topology.

(2) Let $\mathscr{D}$ be a space of Schwartz test functions, e.g., $C_{c}^{\infty}(\Omega)$, given its natural LF-topology, see [Trè06]; then the inclusion

$$
C_{c}^{\infty}(\Omega) \underset{j}{\hookrightarrow} L^{2}(\Omega)
$$

satisfies the condition in lemma 4.2.1.

Corollary 4.2.3. Let $\mathscr{D} \subset L^{2}(\Omega)$ be a subspace satisfying the conditions in lemma 4.2.1; and consider the triple of spaces (4.2.1); then the inner product in $L^{2}(\Omega)$, here denoted $\langle\cdot, \cdot\rangle_{2}$, extends by closure to a sesquilinear function $\langle\cdot, \cdot\rangle$ (which we shall also denote by $\left.\langle\cdot, \cdot\rangle_{2}\right)$ :

$$
\langle\cdot, \cdot\rangle: L^{2}(\Omega) \times \mathscr{D}^{*} \rightarrow \mathbb{C} .
$$

Proof. This is a standard argument based on dual topologies; see [Trè06].

Example 4.2.4 (Application). If $\mathscr{D}=C_{c}^{\infty}(\Omega)$ in $(4.2 .1)$, then $\mathscr{D}^{*}=$ the space of all Schwartz-distributions on $\Omega$, including the Dirac masses. Referring to (4.2.3), we shall write $\left\langle\delta_{x}, f\right\rangle_{2}$ to mean $f(x)$, when $f \in C(\bar{\Omega}) \cap L^{2}(\Omega)$.

Adopting the constructions from Lemma lemma 4.2.1 and Corollary 4.2.3, we now turn to calculus of positive definite functions:

Definition 4.2.5. If $F: \Omega-\Omega \rightarrow \mathbb{C}$ is a function, or a distribution, then we say that $F$ is positive definite iff

$$
\langle F, \bar{\varphi} \otimes \varphi\rangle \geq 0
$$

for all $\varphi \in C_{c}^{\infty}(\Omega)$. The meaning of (4.2.4) is the distribution $K_{F}:=F(x-y)$ acting on $(\bar{\varphi} \otimes \varphi)(x, y):=\overline{\varphi(x)} \varphi(y), x, y \in \Omega$. 
Let

$$
\triangle:=\sum_{j=1}^{k}\left(\frac{\partial}{\partial x_{j}}\right)^{2}
$$

and consider an open domain $\Omega \subset \mathbb{R}^{k}$.

In $\mathscr{H}_{F}$, set

$$
D_{j}^{(F)}\left(F_{\varphi}\right):=F_{\frac{\partial \varphi}{\partial x_{j}}}, \varphi \in C_{c}^{\infty}(\Omega), j=1, \ldots, k .
$$

Then this is a system of commuting skew-Hermitian operators with dense domain in $\mathscr{H}_{F}$.

Lemma 4.2.6. Let $F: \Omega-\Omega \rightarrow \mathbb{C}$ be a positive definite function (or a distribution); and set

$$
M:=-\triangle F
$$

where $\triangle F$ on the RHS in (4.2.7) is in the sense of distributions. Then $M$ is also positive definite and

$$
\left\langle M_{\varphi}, M_{\psi}\right\rangle_{\mathscr{H}_{M}}=\sum_{j=1}^{k}\left\langle D_{j}^{(F)} F_{\varphi}, D_{j}^{(F)} F_{\psi}\right\rangle_{\mathscr{H}_{F}}
$$

for all $\varphi, \psi \in C_{c}^{\infty}(\Omega)$. In particular, setting $\varphi=\psi$ in (4.2.8), we have

$$
\left\|M_{\varphi}\right\|_{\mathscr{H}_{M}}^{2}=\sum_{j=1}^{k}\left\|D_{j}^{(F)} F_{\varphi}\right\|_{\mathscr{H}_{F}}^{2} .
$$

Proof. We must show that $M$ satisfies (4.2.4), i.e., that

$$
\langle M, \bar{\varphi} \otimes \varphi\rangle \geq 0 ;
$$

and moreover that (4.2.8), or equivalently (4.2.7), holds.

For $\operatorname{LHS}_{(4.2 .10)}$, we have

$$
\langle M, \bar{\varphi} \otimes \varphi\rangle=\langle-\triangle F, \bar{\varphi} \otimes \varphi\rangle=-\sum_{j=1}^{k}\left\langle\left(\frac{\partial}{\partial x_{j}}\right)^{2} F, \bar{\varphi} \otimes \varphi\right\rangle ;
$$

and using the action of $\frac{\partial}{\partial x_{j}}$ in the sense of distributions, we get,

$$
\operatorname{LHS}_{(4.2 .10)}=\sum_{j=1}^{k}\left\langle F, \frac{\overline{\partial \varphi}}{\partial x_{j}} \otimes \frac{\partial \varphi}{\partial x_{j}}\right\rangle \underset{(\text { by } \underset{(4.2 .6))}{=}}{=} \sum_{j=1}^{k}\left\|D_{j}^{(F)}\left(F_{\varphi}\right)\right\|_{\mathscr{H}_{F}}^{2} \geq 0,
$$

which yields of the desired conclusions.

Example 4.2.7. For $k=1$, consider the functions $F_{2}$ and $F_{3}$ from Table 3.1.1.

(1) Let $F=F_{2}, \Omega=\left(-\frac{1}{2}, \frac{1}{2}\right)$, then

$$
M=-F^{\prime \prime}=2 \delta
$$

where $\delta$ is the Dirac mass at $x=0$, i.e., $\delta=\delta(x-0)$.

(2) Let $F=F_{3}, \Omega=(-1,1)$, then

$$
M=-F^{\prime \prime}=2 \delta-F
$$

Proof. The proof of the assertions in the two examples follows directly from sections 4.3.2 and 4.3.3. 
Now we return to the p.d. function $F: \Omega-\Omega \rightarrow \mathbb{C}$. Suppose $A: L^{2}(\Omega) \rightarrow$ $L^{2}(\Omega)$ is an unbounded positive linear operator, i.e., $A \geq c>0$, for some constant $c$. Further assume that $A^{-1}$ has the integral kernel (Green's function) $F$, i.e.,

$$
\left(A^{-1} f\right)(x)=\int_{\Omega} F(x-y) f(y) d y, \forall f \in L^{2}(\Omega) .
$$

For all $x \in \Omega$, define

$$
F_{x}(\cdot):=F(x-\cdot) .
$$

Here $F_{x}$ is the fundamental solution to the following equation

$$
A u=f
$$

where $u \in \operatorname{dom}(A)$, and $f \in L^{2}(\Omega)$. Hence, in the sense of distribution, we have

$$
\begin{aligned}
A F_{x}(\cdot) & =\delta_{x} \\
& \mathbb{1} \\
A\left(\int_{\Omega} F(x, y) f(y) d y\right) & =\int\left(A F_{x}(y)\right) f(y) d y \\
& =\int \delta_{x}(y) f(y) d y \\
& =f(x) .
\end{aligned}
$$

Note that $A^{-1} \geq 0$ iff $F$ is a p.d. kernel.

Let $\mathscr{H}_{A}=$ the completion of $C_{c}^{\infty}(\Omega)$ in the bilinear form

$$
\langle f, g\rangle_{A}:=\langle A f, g\rangle_{2}
$$

where the RHS extends the inner product in $L^{2}(\Omega)$ as in (4.2.3).

Lemma 4.2.8. $\mathscr{H}_{A}$ is a RKHS and the reproducing kernel is $F_{x}$.

Proof. Since $A \geq c>0$, in the usual ordering of Hermitian operator, (4.2.15) is a well-defined inner product, so $\mathscr{H}_{A}$ is a Hilbert space. For the reproducing property, we check that

$$
\left\langle F_{x}, g\right\rangle_{A}=\left\langle A F_{x}, g\right\rangle_{2}=\left\langle\delta_{x}, g\right\rangle_{2}=g(x) .
$$

Lemma 4.2.9. Let $\mathscr{H}_{F}$ be the RKHS corresponding to $F$, i.e., the completion of $\operatorname{span}\left\{F_{x}: x \in \Omega\right\}$ in the inner product

$$
\left\langle F_{y}, F_{x}\right\rangle_{F}:=F_{x}(y)=F(x-y)
$$

extending linearly. Then we have the isometric embedding $\mathscr{H}_{F} \hookrightarrow \mathscr{H}_{A}$, via the map,

$$
F_{x} \mapsto F_{x} .
$$

Proof. We check directly that

$$
\begin{aligned}
& \left\|F_{x}\right\|_{F}^{2}=\left\langle F_{x}, F_{x}\right\rangle_{F}=F_{x}(x)=F(0) \\
& \left\|F_{x}\right\|_{A}^{2}=\left\langle F_{x}, F_{x}\right\rangle_{A}=\left\langle A F_{x}, F_{x}\right\rangle_{L^{2}}=\left\langle\delta_{x}, F_{x}\right\rangle_{L^{2}}=F_{x}(x)=F(0) .
\end{aligned}
$$


REmark 4.2.10. Now consider $\mathbb{R}$, and let $\Omega=(0, a)$. Recall the Mercer operator

$$
\begin{aligned}
T_{F}: & L^{2}(\Omega) \rightarrow L^{2}(\Omega), \text { by } \\
\left(T_{F} g\right)(x) & :=\int_{0}^{a} F_{x}(y) g(y) d y \\
& =\left\langle F_{x}, g\right\rangle_{2}, \forall g \in L^{2}(0, a) .
\end{aligned}
$$

By Corollary 4.1.2, $T_{F}$ can be diagonalized in $L^{2}(0, a)$ by

$$
T_{F} \xi_{n}=\lambda_{n} \xi_{n}, \lambda_{n}>0
$$

where $\left\{\xi_{n}\right\}_{n \in \mathbb{N}}$ is an ONB in $L^{2}(0, a)$; further $\xi_{n} \subset \mathscr{H}_{F}$, for all $n \in \mathbb{N}$.

From (4.2.18), we then have

$$
\left\langle F_{x}, \xi_{n}\right\rangle_{2}=\lambda_{n} \xi_{n}(x) .
$$

Applying $A$ on both sides of (4.2.19) yields

$$
\begin{aligned}
\operatorname{LHS}_{(4.2 .19)} & =\left\langle A F_{x}, \xi_{n}\right\rangle_{2}=\left\langle\delta_{x}, \xi_{n}\right\rangle_{2}=\xi_{n}(x) \\
\operatorname{RHS}_{(4.2 .19)} & =\lambda_{n}\left(A \xi_{n}\right)(x)
\end{aligned}
$$

therefore, $A \xi_{n}=\frac{1}{\lambda_{n}} \xi_{n}$, i.e.,

$$
A=T_{F}^{-1}
$$

Consequently,

$$
\left\langle\xi_{n}, \xi_{m}\right\rangle_{A}=\left\langle A \xi_{n}, \xi_{m}\right\rangle_{2}=\frac{1}{\lambda_{n}}\left\langle\xi_{n}, \xi_{m}\right\rangle_{2}=\frac{1}{\lambda_{n}} \delta_{n, m} .
$$

And we conclude that $\left\{\sqrt{\lambda_{n}} \xi_{n}\right\}_{n \in \mathbb{N}}$ is an ONB in $\mathscr{H}_{A}=\mathscr{H}_{T_{F}^{-1}}$.

See section 3.2, where $F=$ Polya extension of $F_{3}$, and a specific construction of $\mathscr{H}_{T_{F}^{-1}}$.

4.2.1. Connection to the Energy Space Hilbert Space. Now consider $A=1-\triangle$ defined on $C_{c}^{\infty}(\Omega)$. There is a connection between the RKHS $\mathscr{H}_{A}$ and the energy space as follows:

For $f, g \in \mathscr{H}_{A}$, we have (restricting to real valued functions),

$$
\begin{aligned}
\langle f, g\rangle_{A} & =\langle(1-\triangle) f, g\rangle_{L^{2}} \\
& =\int_{\Omega} f g-\int_{\Omega}(\triangle f) g \\
& =\underbrace{\int_{\Omega} f g+\int_{\Omega} D f \cdot D g}_{\text {energy inner product }}+\text { boundary corrections; }
\end{aligned}
$$

So we define

$$
\langle f, g\rangle_{\text {Energy }}:=\int_{\Omega} f g+\int_{\Omega} D f \cdot D g
$$

and then

$$
\langle f, g\rangle_{A}=\langle f, g\rangle_{\text {Energy }}+\text { boundary corrections. }
$$

Remark 4.2.11. The $A$-inner product on the LHS of (4.2.22) incorporates the boundary information. 
Example 4.2.12. Consider $L^{2}(0,1), F(x)=\left.e^{-|x|}\right|_{(-1,1)}$, and $A=\frac{1}{2}(1-$ $\left.\left(\frac{d}{d x}\right)^{2}\right)$. We have

$$
\begin{aligned}
\langle f, g\rangle_{A} & =\frac{1}{2}\left\langle f-f^{\prime \prime}, g\right\rangle_{L^{2}} \\
& =\frac{1}{2} \int_{0}^{1} f g-\frac{1}{2} \int_{0}^{1} f^{\prime \prime} g \\
& =\frac{1}{2}\left(\int_{0}^{1} f g+\int_{0}^{1} f^{\prime} g^{\prime}\right)+\frac{\left(f^{\prime} g\right)(0)-\left(f^{\prime} g\right)(1)}{2} \\
& =\langle f, g\rangle_{\text {Energy }}+\frac{\left(f^{\prime} g\right)(0)-\left(f^{\prime} g\right)(1)}{2} .
\end{aligned}
$$

Here, the boundary term

$$
\frac{\left(f^{\prime} g\right)(0)-\left(f^{\prime} g\right)(1)}{2}
$$

contains the inward normal derivative of $f^{\prime}$ at $x=0$ and $x=1$.

(1) We proceed to check the reproducing property w.r.t. the $A$-inner product:

$$
2\left\langle e^{-|x-\cdot|}, g\right\rangle_{\text {Energy }}=\int_{0}^{1} e^{-|x-y|} g(y) d y+\int_{0}^{1}\left(\frac{d}{d y} e^{-|x-y|}\right) g^{\prime}(y) d y
$$

where

$$
\begin{aligned}
& \int_{0}^{1}\left(\frac{d}{d y} e^{-|x-y|}\right) g^{\prime}(y) d y \\
= & \int_{0}^{x} e^{-(x-y)} g^{\prime}(y) d y-\int_{x}^{1} e^{-(y-x)} g^{\prime}(y) d y \\
= & 2 g(x)-g(0) e^{-x}-g(1) e^{-(1-x)}-\int_{0}^{1} e^{-|x-y|} g(y) d y ;
\end{aligned}
$$

it follows that

$$
\left\langle e^{-|x-\cdot|}, g\right\rangle_{\text {Energy }}=g(x)-\frac{g(0) e^{-x}+g(1) e^{-(1-x)}}{2}
$$

(2) It remains to check the boundary term in (4.2.24) comes from the inward normal derivative of $e^{-|x-\cdot|}$. Indeed, set $f(\cdot)=e^{-|x-\cdot|}$ in (4.2.23), then

$$
f^{\prime}(0)=e^{-x}, \quad f^{\prime}(1)=-e^{-(1-x)}
$$

therefore,

$$
\frac{\left(f^{\prime} g\right)(0)-\left(f^{\prime} g\right)(1)}{2}=\frac{e^{-x} g(0)+e^{-(1-x)} g(1)}{2} .
$$

Example 4.2.13. Consider $L^{2}\left(0, \frac{1}{2}\right), F(x)=1-|x|$ with $|x|<\frac{1}{2}$, and let $A=-\frac{1}{2}\left(\frac{d}{d x}\right)^{2}$. Then the $A$-inner product yields

$$
\begin{aligned}
\langle f, g\rangle_{A} & =-\frac{1}{2}\left\langle f^{\prime \prime}, g\right\rangle_{L^{2}} \\
& =\frac{1}{2} \int_{0}^{\frac{1}{2}} f^{\prime} g^{\prime}-\frac{\left(f^{\prime} g\right)\left(\frac{1}{2}\right)-\left(f^{\prime} g\right)(0)}{2} \\
& =\langle f, g\rangle_{\text {Energy }}+\frac{\left(f^{\prime} g\right)(0)-\left(f^{\prime} g\right)\left(\frac{1}{2}\right)}{2}
\end{aligned}
$$


where we set

$$
\langle f, g\rangle_{\text {Energy }}:=\frac{1}{2} \int_{0}^{\frac{1}{2}} f^{\prime} g^{\prime} ;
$$

and the corresponding boundary term is

$$
\frac{\left(f^{\prime} g\right)(0)-\left(f^{\prime} g\right)\left(\frac{1}{2}\right)}{2}
$$

(1) To check the reproducing property w.r.t. the $A$-inner product: Set

$F_{x}(y):=1-|x-y|, x, y \in\left(0, \frac{1}{2}\right) ;$ then

$$
\begin{aligned}
\left\langle F_{x}, g\right\rangle_{\text {Energy }} & =\frac{1}{2} \int_{0}^{\frac{1}{2}} F_{x}(y)^{\prime} g^{\prime}(y) d y \\
& ==\frac{1}{2}\left(\int_{0}^{x} g^{\prime}(y) d y-\int_{x}^{\frac{1}{2}} g^{\prime}(y) d y\right) \\
& =g(x)-\frac{g(0)+g\left(\frac{1}{2}\right)}{2} .
\end{aligned}
$$

(2) Now we check the second term on the RHS of (4.2.26) contains the inward normal derivative of $F_{x}$. Note that

$$
\begin{aligned}
F_{x}^{\prime}(0) & =\left.\frac{d}{d y}\right|_{y=0}(1-|x-y|)=1 \\
F_{x}^{\prime}\left(\frac{1}{2}\right) & =\left.\frac{d}{d y}\right|_{y=\frac{1}{2}}(1-|x-y|)=-1
\end{aligned}
$$

Therefore,

$$
\frac{\left(f^{\prime} g\right)(0)-\left(f^{\prime} g\right)\left(\frac{1}{2}\right)}{2}=\frac{g(0)+g\left(\frac{1}{2}\right)}{2}
$$

which verifies the boundary term in (4.2.25).

\subsection{The RKHSs for the Two Examples $F_{2}$ and $F_{3}$ in Table 3.1.1}

In this section, we revisit cases $F_{2}$, and $F_{3}$ (from Table 3.1.1) and their associated RKHSs. We show that they are (up to isomorphism) also the Hilbert spaces used in stochastic integration for Brownian motion, and for the OrnsteinUhlenbeck process (see e.g., [Hid80]), respectively. As reproducing kernel Hilbert spaces, they have an equivalent and more geometric form, of use in for example analysis of Gaussian processes. Analogous results for the respective RKHSs also hold for other positive definite function systems $(F, \Omega)$, but for the present two examples $F_{2}$, and $F_{3}$, the correspondences involved are explicit. As a bonus, we get an easy and transparent proof that the deficiency-indices for the respective operators $D^{(F)}$ are $(1,1)$ in both these examples.

The purpose of the details below are two-fold. First we show that the respective RKHSs corresponding to $F_{2}$ and $F_{3}$ in Table 3.1 .1 are naturally isomorphic to more familiar RKHSs which are used in the study of Gaussian processes, see e.g., [AJL11, AL10, AJ12]; and secondly, to give an easy (and intuitive) proof that the deficiency indices in these two cases are $(1,1)$. Recall for each p.d. function $F$ in an interval $(-a, a)$, we study

$$
D^{(F)}\left(F_{\varphi}\right):=F_{\varphi^{\prime}}, \varphi \in C_{c}^{\infty}(0, a)
$$

as a skew-Hermitian operator in $\mathscr{H}_{F}$; see Lemma 2.4.2. 


\subsubsection{Green's Functions.}

\section{LEMMA 4.3.1.}

(1) For $F_{2}(x)=1-|x|,|x|<\frac{1}{2}$, let $\varphi \in C_{c}^{\infty}\left(0, \frac{1}{2}\right)$, then $u(x):=\left(T_{F_{2}} \varphi\right)(x)$ satisfies

Hence,

$$
\varphi=-\frac{1}{2}\left(\frac{d}{d x}\right)^{2} u
$$

$$
T_{F_{2}}^{-1} \supset-\left.\frac{1}{2}\left(\frac{d}{d x}\right)^{2}\right|_{C_{c}^{\infty}\left(0, \frac{1}{2}\right)} .
$$

(2) For $F_{3}(x)=e^{-|x|},|x|<1$, let $\varphi \in C_{c}^{\infty}(0,1)$, then

$$
\varphi=\frac{1}{2}\left(I-\left(\frac{d}{d x}\right)^{2}\right) u \text {. }
$$

Hence,

$$
\left.T_{F_{3}}^{-1} \supset \frac{1}{2}\left(I-\left(\frac{d}{d x}\right)^{2}\right)\right|_{C_{c}^{\infty}(0,1)} .
$$

Proof. The computation for $F=F_{2}$ is as follows: Let $\varphi \in C_{c}^{\infty}\left(0, \frac{1}{2}\right)$, then

$$
\begin{aligned}
u(x)=\left(T_{F_{2}} \varphi\right)(x) & =\int_{0}^{\frac{1}{2}} \varphi(y)(1-|x-y|) d y \\
& =\int_{0}^{x} \varphi(y)(1-(x-y)) d y+\int_{x}^{\frac{1}{2}} \varphi(y)(1-(y-x)) d y
\end{aligned}
$$

and

$$
\begin{gathered}
\int_{0}^{x} \varphi(y)(1-(x-y)) d y=\int_{0}^{x} \varphi(y) d y-x \int_{0}^{x} \varphi(y) d y+\int_{0}^{x} y \varphi(y) d y \\
u^{\prime}(x)=-\int_{0}^{x} \varphi(y)+\varphi(x)+\int_{x}^{\frac{1}{2}} \varphi(y) d y-\varphi(x) \\
u^{\prime \prime}(x)=-2 \varphi(x) .
\end{gathered}
$$

Thus, $\varphi=-\frac{1}{2} u^{\prime \prime}$, and the desired result follows.

For $F_{3}$, let $\varphi \in C_{c}^{\infty}(0,1)$, then

$$
\begin{aligned}
u(x)=\left(T_{F_{3}} \varphi\right)(x) & =\int_{0}^{1} e^{-|x-y|} \varphi(y) d y \\
& =\int_{0}^{x} e^{-(x-y)} \varphi(y) d y+\int_{x}^{1} e^{-(y-x)} \varphi(y) d y
\end{aligned}
$$

Now,

$$
\begin{aligned}
u^{\prime}(x)=\left(T_{F_{3}} \varphi\right)^{\prime}(x)= & -e^{-x} \int_{0}^{x} e^{-y} \varphi(y) d y+\varphi(x) \\
& +e^{x} \int_{x}^{1} e^{-y} \varphi(y) d y-\varphi(x) \\
= & -e^{-x} \int_{0}^{x} e^{y} \varphi(y) d y+e^{x} \int_{x}^{1} e^{-y} \varphi(y) d y \\
u^{\prime \prime}= & e^{-x} \int_{0}^{x} e^{y} \varphi(y) d y-\varphi(x)
\end{aligned}
$$




$$
\begin{aligned}
& +e^{x} \int_{x}^{1} e^{-y} \varphi(y) d y-\varphi(x) \\
= & -2 \varphi+\int_{0}^{1} e^{-|x-y|} \varphi(y) d y \\
= & -2 \varphi+T_{F_{3}}(\varphi) ;
\end{aligned}
$$

and then

$$
\begin{aligned}
u^{\prime \prime}(x)= & e^{-x} \int_{0}^{x} e^{y} \varphi(y) d y-\varphi(x) \\
& +e^{x} \int_{x}^{1} e^{-y} \varphi(y) d y-\varphi(x) \\
= & -2 \varphi(x)+\int_{0}^{1} e^{-|x-y|} \varphi(y) d y \\
= & -2 \varphi+u(x) .
\end{aligned}
$$

Thus, $\varphi=\frac{1}{2}\left(u-u^{\prime \prime}\right)=\frac{1}{2}\left(I-\frac{1}{2}\left(\frac{d}{d x}\right)^{2}\right) u$. This proves (4.3.4).

Summary 4.3.2 (Conclusions for the two examples). The computation for $F=$ $F_{2}$ is as follows: If $\varphi \in L^{2}\left(0, \frac{1}{2}\right)$, then $u(x):=\left(T_{F} \varphi\right)(x)$ satisfies

$$
\left(F_{2}\right) \quad \varphi=\frac{1}{2}\left(-\left(\frac{d}{d x}\right)^{2}\right) u ;
$$

while, for $F=F_{3}$, the corresponding computation is as follows: If $\varphi \in L^{2}(0,1)$, then $u(x)=\left(T_{F} \varphi\right)(x)$ satisfies

$$
\left(F_{3}\right) \quad \varphi=\frac{1}{2}\left(I-\left(\frac{d}{d x}\right)^{2}\right) u ;
$$

For the operator $D^{(F)}$, in the case of $F=F_{2}$, it follows that the Mercer operator $T_{F}$ plays the following role: $T_{F}^{-1}$ is a selfadjoint extension of $-\frac{1}{2}\left(D^{(F)}\right)^{2}$. In the case of $F=F_{3}$ the corresponding operator $T_{F}^{-1}$ (in the RKHS $\mathscr{H}_{F_{3}}$ ) is a selfadjoint extension of $\frac{1}{2}\left(I-\left(D^{(F)}\right)^{2}\right)$; in both cases, they are the Friedrichs extensions.

REMARK 4.3.3. When solving boundary values for elliptic operators in a bounded domain, say $\Omega \subset \mathbb{R}^{n}$, one often ends up with Green's functions (= integral kernels) which are positive definite kernels, so $K(x, y)$, defined on $\Omega \times \Omega$, not necessarily of the form $K(x, y)=F(x-y)$.

But many of the questions we ask in the special case of p.d. functions, so when the kernel is $K(x, y)=F(x-y)$ will also make sense for p.d. kernels.

4.3.2. The Case of $F_{2}(x)=1-|x|, x \in\left(-\frac{1}{2}, \frac{1}{2}\right)$. Let $F=F_{2}$. Fix $x \in\left(0, \frac{1}{2}\right)$, and set

$$
F_{x}(y)=F(x-y), \text { for } x, y \in\left(0, \frac{1}{2}\right)
$$

where $F_{x}(\cdot)$ and its derivative (in the sense of distributions) are as in Figure 4.3.1 (sketched for two values of $x$ ).

Consider the Hilbert space

$$
\mathscr{H}_{F}:=\left\{\begin{array}{ll}
h ; & \begin{array}{l}
\text { continuous on }\left(0, \frac{1}{2}\right), \text { and } h^{\prime}=\frac{d h}{d x} \in L^{2}\left(0, \frac{1}{2}\right) \\
\text { where the derivative is in the weak sense }
\end{array}
\end{array}\right\}
$$



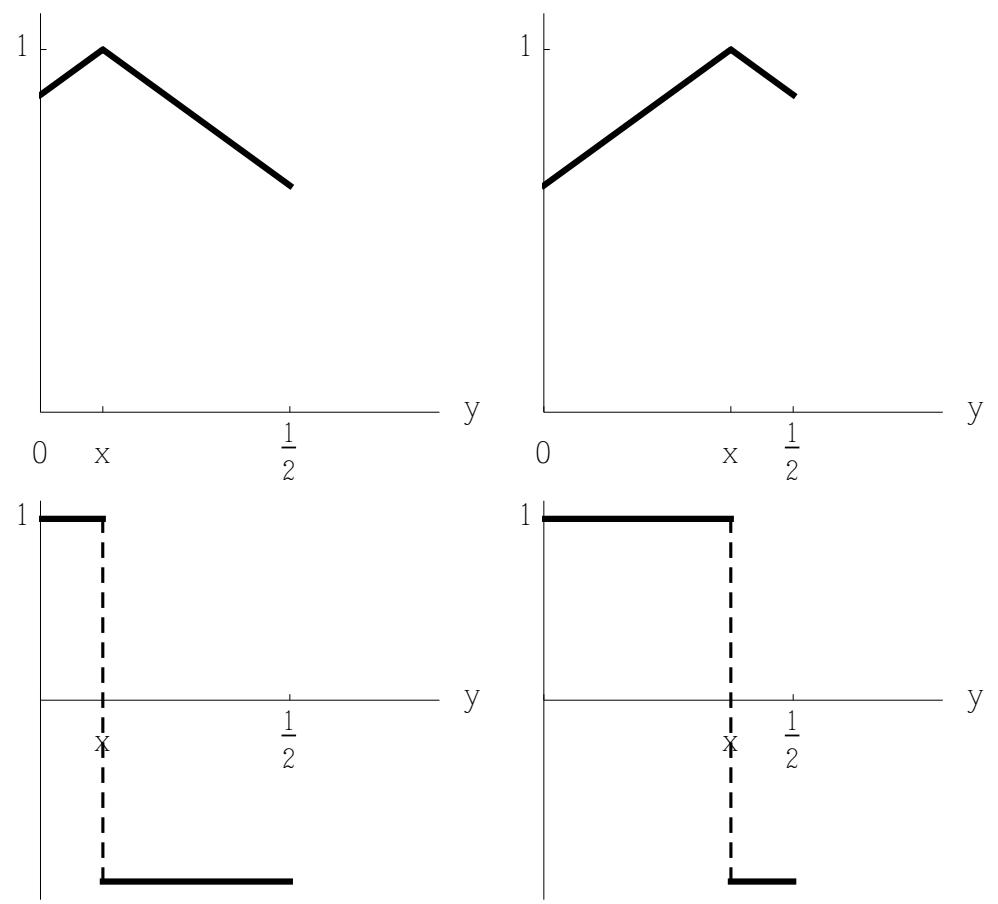

Figure 4.3.1. The kernel $F_{x}$ and its derivative (the case of $F_{2}$ )

modulo constants; and let the norm, and inner-product, in $\mathscr{H}_{F}$ be given by

$$
\|h\|_{\mathscr{H}_{F}}^{2}=\frac{1}{2} \int_{0}^{\frac{1}{2}}\left|h^{\prime}(x)\right|^{2} d x+\int_{\partial \Omega} \overline{h_{n}} h d \beta
$$

On the RHS of (4.3.8), $d \beta$ denotes the corresponding boundary measure, and $h_{n}$ is the inward normal derivative of $h$. See Theorem 4.3.4 below.

Then the reproducing kernel property is as follows:

$$
\left\langle F_{x}, h\right\rangle_{\mathscr{H}_{F}}=h(x), \forall h \in \mathscr{H}_{F}, \forall x \in\left(0, \frac{1}{2}\right) ;
$$

and it follows that $\mathscr{H}_{F}$ is naturally isomorphic to the RKHS for $F_{2}$ from section 2.4.

Theorem 4.3.4. The boundary measure for $F=F_{2}$ (see (4.3.8)) is

$$
\beta=\frac{1}{2}\left(\delta_{0}+\delta_{1 / 2}\right)
$$

Proof. Set

$$
E(\xi):=\frac{1}{2} \int_{0}^{\frac{1}{2}}\left|\xi^{\prime}(x)\right|^{2} d x, \forall \xi \in \mathscr{H}_{F} .
$$

And let $F_{x}(\cdot):=1-|x-\cdot|$ defined on $\left[0, \frac{1}{2}\right]$, for all $x \in\left(0, \frac{1}{2}\right)$. Then

$$
\begin{aligned}
E\left(F_{x}, \xi\right) & =\frac{1}{2} \int_{0}^{\frac{1}{2}} F_{x}^{\prime}(y) \xi^{\prime}(y) d y \\
& =\frac{1}{2}\left(\int_{0}^{x} \xi^{\prime}(y) d y-\int_{x}^{\frac{1}{2}} \xi^{\prime}(y) d y\right)
\end{aligned}
$$




$$
=\xi(x)-\frac{\xi(0)+\xi\left(\frac{1}{2}\right)}{2} . \text { (see Fig. 4.3.1) }
$$

Since

$$
\|\xi\|_{\mathscr{H}_{F}}^{2}=E(\xi)+\int|\xi|^{2} d \beta
$$

we get

We conclude that

$$
\left\langle F_{x}, \xi\right\rangle_{\mathscr{H}_{F}}=\xi(x), \forall \xi \in \mathscr{H}_{F} .
$$

$$
\langle\xi, \eta\rangle_{\mathscr{H}_{F}}=E(\xi, \eta)+\int_{\partial \Omega} \overline{\xi_{n}} \eta d \beta
$$

note the boundary in this case consists two points, $x=0$ and $x=\frac{1}{2}$.

REMARK 4.3.5. The energy form in (4.3.10) also defines a RKHS (Wiener's energy form for Brownian motion, see figure 4.3.3) as follows:

On the space of all continuous functions, $\mathscr{C}\left(\left[0, \frac{1}{2}\right]\right)$, set

$$
\mathscr{H}_{\mathscr{E}}:=\left\{f \in \mathscr{C}\left(\left[0, \frac{1}{2}\right]\right) \mid \mathscr{E}(f)<\infty\right\}
$$

modulo constants, where

$$
\mathscr{E}(f)=\int_{0}^{\frac{1}{2}}\left|f^{\prime}(x)\right|^{2} d x
$$

For $x \in\left[0, \frac{1}{2}\right]$, set

$$
E_{x}(y)=x \wedge y=\min (x, y), y \in\left(0, \frac{1}{2}\right) ;
$$

see Figure 4.3.2; then $E_{x} \in \mathscr{H}_{\mathscr{E}}$, and

$$
\left\langle E_{x}, f\right\rangle_{\mathscr{H}_{\mathscr{E}}}=f(x), \forall f \in \mathscr{H}_{\mathscr{E}}, \forall x \in\left[0, \frac{1}{2}\right] .
$$

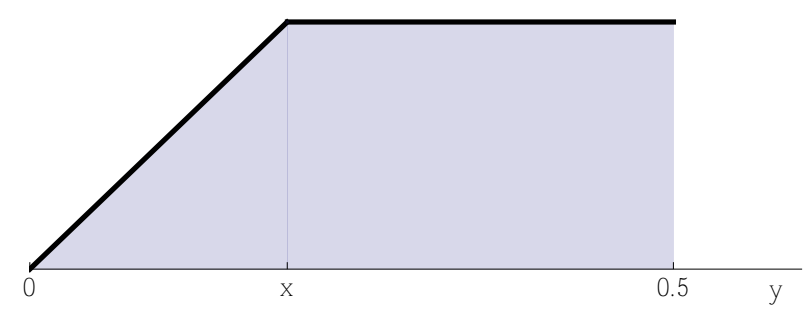

Figure 4.3.2. The covariance function $E_{x}(\cdot)=\min (x, \cdot)$ of Brownian motion.

Proof. For the reproducing property (4.3.14): Let $f$ and $x$ be as stated; then

$$
\begin{aligned}
\left\langle E_{x}, f\right\rangle_{\mathscr{H}_{B}} & =\int_{0}^{\frac{1}{2}} E_{x}^{\prime}(y) f^{\prime}(y) d y \\
& =\int_{0}^{\frac{1}{2}} \chi_{[0, x]}(y) f^{\prime}(y) d y \\
& =\int_{0}^{x} f^{\prime}(y) d y=f(x)-f(0) .
\end{aligned}
$$

Note in (4.3.12) we define $\mathscr{H}_{\mathscr{E}}$ modulo constants; alternatively, we may stipulate $f(0)=0$. 
Note that the Brownian motion RKHS is not defined by a p.d. function, but rather by a p.d. kernel. Nonetheless the remark explains its connection to our present RKHS $\mathscr{H}_{F}$ which is defined by the p.d. function, namely the p.d. function $F_{2}$.

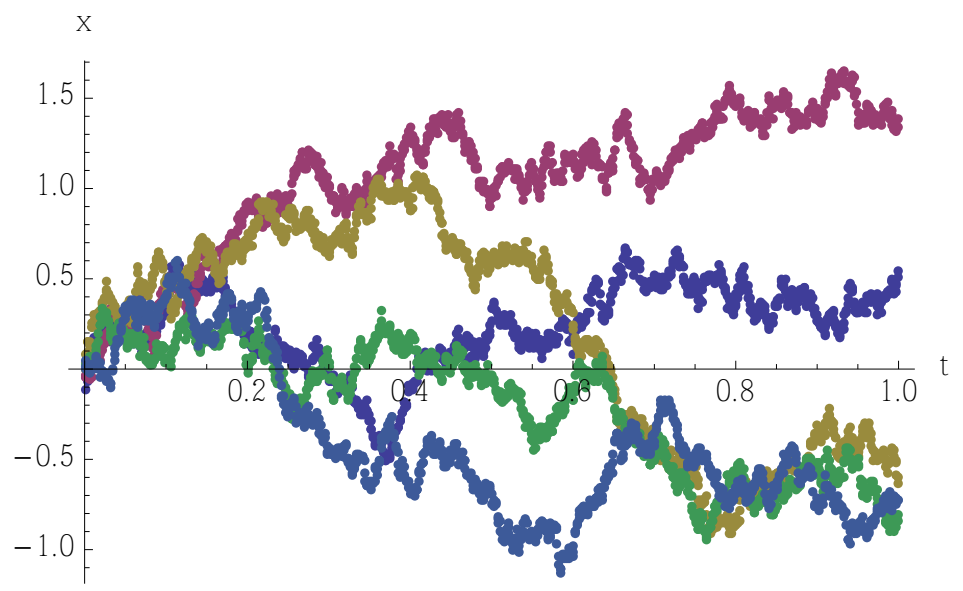

Figure 4.3.3. Monte-Carlo simulation of Brownian motion starting at $x=0$, with 5 sample paths.

\section{Pinned Brownian Motion.}

We illustrate the boundary term in Theorem 4.3.4, eq. (4.3.11) with pinned Brownian motion (also called "Brownian bridge.") In order to simplify constructions, we pin the Brownian motion at the following two points in $(t, x)$ space, $(t, x)=(0,0)$, and $(t, x)=(1,1)$; see Figure 4.3.4. To simplify computations further, we restrict attention to real valued functions only.

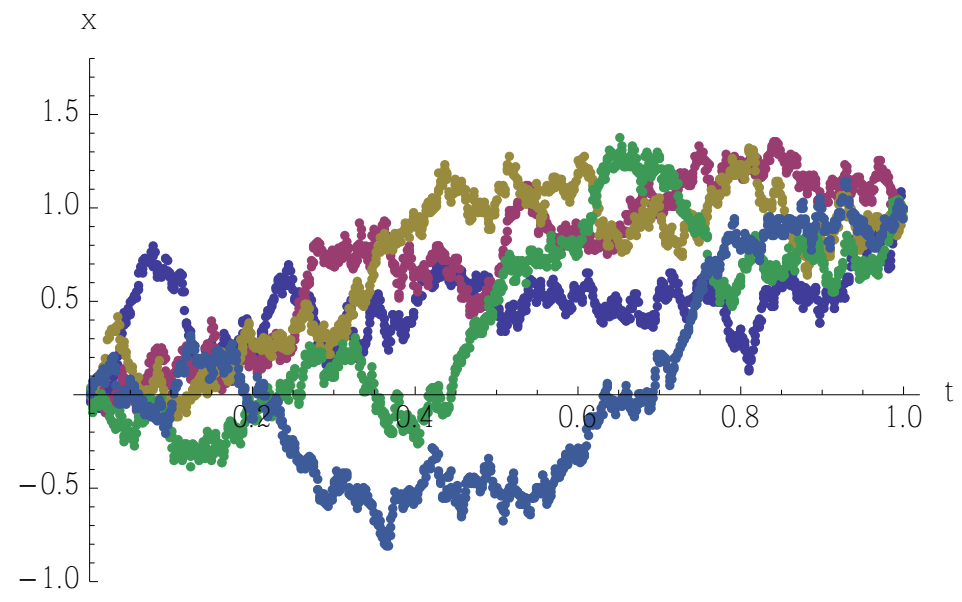

Figure 4.3.4. Monte-Carlo simulation of Brownian bridge pinned at $(0,0)$ and $(1,1)$, with 5 sample paths. 
For background on stochastic processes, the book [IW89] serves our purpose; see especially p. 243-244.

For the pinning down the process $X_{t}$ at the two points $(0,0)$ and $(1,1)$ as in Figure 4.3.4, we have

$$
X_{t}=t+(1-t) \int_{0}^{t} \frac{d B_{s}}{1-s}, 0<t<1
$$

where $d B_{s}$ on the RHS of (4.3.15) refers to the standard Brownian motion $d B_{s}$, and the second term in (4.3.15) is the corresponding Ito-integral; so we have

$$
\begin{aligned}
\mathbb{E}\left(\int_{0}^{t} \frac{d B_{s}}{1-s}\right) & =0, \text { and } \\
\mathbb{E}\left(\left|\int_{0}^{t} \frac{d B_{s}}{1-s}\right|^{2}\right) & =\int_{0}^{t} \frac{d s}{(1-s)^{2}}=\frac{t}{1-t} ;
\end{aligned}
$$

where $\mathbb{E}(\cdots)=$ expectation with respect to the underlying probability space $(\Omega, \mathscr{F}, \mathbb{P})$, i.e., $\mathbb{E}(\cdots)=\int_{\Omega} \cdots d \mathbb{P}$.

Hence, for the mean and covariance, of the process $X_{t}$ in (4.3.15), we get

$$
\begin{gathered}
\mathbb{E}\left(X_{t}\right)=t, \forall t, 0<t<1 ; \text { and } \\
\operatorname{Cov}\left(X_{t} X_{s}\right)=\mathbb{E}\left(\left(X_{t}-t\right)\left(X_{s}-s\right)\right)=s \wedge t-s t ;
\end{gathered}
$$

where $s \wedge t=\min (s, t)$, and $s, t \in(0,1)$.

And it follows in particular that the function on the RHS in eq (4.3.19) is a positive definite kernel. Its connection to $F_{2}$ is given in the next lemma.

Now return to the p.d. function $F=F_{2}$; i.e., $F(t)=1-|t|$, and therefore, $F(s-t)=1-|s-t|, s, t \in(0,1)$, and let $\mathscr{H}_{F}$ be the corresponding RKHS.

Let $\left\{F_{t}\right\}_{t \in(0,1)}$ denote the kernels in $\mathscr{H}_{F}$, i.e., $\left\langle F_{t}, g\right\rangle_{\mathscr{H}_{F}}=g(t), t \in(0,1)$, $g \in \mathscr{H}_{F}$.

Lemma 4.3.6. Let $\left(X_{t}\right)_{t \in(0,1)}$ denote the pinned Brownian motion (4.3.15); then

$$
\left\langle F_{s}, F_{t}\right\rangle_{\mathscr{H}_{F}}=\operatorname{Cov}\left(X_{s} X_{t}\right)
$$

see (4.3.19); and

while the boundary term

$$
\left\langle F_{s}, F_{t}\right\rangle_{\text {energy }}=s \wedge t
$$

$$
b d r(s, t)=-s t .
$$

Proof. With our normalization from Fig 4.3.4, we must take the energy form as follows:

$$
\langle f, g\rangle_{\text {energy }}=\int_{0}^{1} f^{\prime}(x) g^{\prime}(x) d x .
$$

Set $F_{s}(y)=s \wedge y$, see Figure 4.3.2. For the distributional derivative we have

$$
F_{s}^{\prime}(y)=\chi_{[0, s]}(y)= \begin{cases}1 & \text { if } 0 \leq y \leq s \\ 0 & \text { else }\end{cases}
$$

then

$$
\left\langle F_{s}, F_{t}\right\rangle_{\text {energy }}=\int_{0}^{1} F_{s}^{\prime}(y) F_{t}^{\prime}(y) d y
$$




$$
\begin{aligned}
& =\int_{0}^{1} \chi_{[0, s]}(y) \chi_{[0, t]}(y) d y \\
& =|[0, s] \cap[0, t]|_{\text {Lebesgue measure }} \\
& =s \wedge t .
\end{aligned}
$$

The desired conclusions (4.3.21)-(4.3.22) in the lemma now follow. See Remark 4.3.5.

The verification of (4.3.20) uses Ito-calculus as follows: Note that (4.3.15) for $X_{t}$ is the solution to the following Ito-equation:

$$
d X_{t}=\left(\frac{X_{t}-1}{t-1}\right) d t+d B_{t}
$$

and by Ito's lemma, therefore,

$$
\left(d X_{t}\right)^{2}=\left(d B_{t}\right)^{2} .
$$

As a result, if $f: \mathbb{R} \rightarrow \mathbb{R}$ is a function in the energy-Hilbert space defined from (4.3.23), then

$$
\mathbb{E}\left(\left|(d f)\left(X_{t}\right)\right|^{2}\right)=\mathbb{E}\left(\left|(d f)\left(B_{t}\right)\right|^{2}\right)=\int_{0}^{1}\left|f^{\prime}(t)\right|^{2} d t=\mathscr{E}_{2}(f) .
$$

REMARK 4.3.7. In (4.3.24)-(4.3.25), we use standard conventions for Brownian motions $B_{t}$ : Let $(\Omega, \mathscr{F}, \mathbb{P})$ be a choice of probability space for $\left\{B_{t}\right\}_{t \in \mathbb{R}}$, (or $t \in$ $[0,1]$.) With $E(\cdots)=\int_{\Omega} \cdots d \mathbb{P}$, we have

$$
E\left(B_{s} B_{t}\right)=s \wedge t=\min (s, t), t \in[0,1] .
$$

If $f: \mathbb{R} \rightarrow \mathbb{R}$ is a $C^{1}$-function, we write $f\left(B_{t}\right)$ for $f \circ B_{t}$; and $d f\left(B_{t}\right)$ refers to Ito-calculus.

4.3.3. The Case of $F_{3}(x)=e^{-|x|}, x \in(-1,1)$. Let $F_{x}(y)=F(x-y)$, for all $x, y \in(0,1)$; and consider the Hilbert space

$$
\mathscr{H}_{F}:=\left\{\begin{array}{ll}
h ; & \text { continuous on }(0,1), h \in L^{2}(0,1), \text { and } \\
\text { the weak derivative } h^{\prime} \in L^{2}(0,1)
\end{array}\right\} ;
$$

and let the $\mathscr{H}_{F}$-norm, and inner product, be given by

$$
\|h\|_{\mathscr{H}_{F}}=\frac{1}{2}\left(\int_{0}^{1}\left|h^{\prime}(x)\right|^{2} d x+\int_{0}^{1}|h(x)|^{2} d x\right)+\int_{\partial \Omega} \overline{h_{n}} h d \beta .
$$

Here, $d \beta$ on the RHS of (4.3.28) denotes the corresponding boundary measure, and $h_{n}$ is the inward normal derivative of $h$. See Theorem 4.3.9 below.

Then a direct verification yields:

$$
\left\langle F_{x}, h\right\rangle_{\mathscr{H}_{F}}=h(x), \forall h \in \mathscr{H}_{F}, \forall x \in(0,1) ;
$$

and it follows that $\mathscr{H}_{F}$ is naturally isomorphic to RKHS for $F_{3}$ from section 2.4 .

For details of (4.3.29), see also [Jør81].

Corollary 4.3.8. In both $\mathscr{H}_{F_{i}}, i=2,3$, the deficiency indices are $(1,1)$.

PRoOF. In both cases, we are referring to the skew-Hermitian operator $D^{\left(F_{i}\right)}$ in $\mathscr{H}_{F i}, i=2,3$; see (4.3.1) above. But it follows from (4.3.8) and (4.3.28) for the respective inner products, that the functions $e^{ \pm x}$ have finite positive norms in the respective RKHSs. 
Theorem 4.3.9. Let $F=F_{3}$ as before. Consider the energy Hilbert space

$$
\mathscr{H}_{E}:=\left\{\begin{array}{c}
f \in C[0,1] \mid f, f^{\prime} \in L^{2}(0,1) \text { where } \\
f^{\prime} \text { is the weak derivative of } f
\end{array}\right\}
$$

with

$$
\begin{aligned}
\langle f, g\rangle_{E} & =\frac{1}{2}\left(\int_{0}^{1} \overline{f^{\prime}(x)} g^{\prime}(x) d x+\int_{0}^{1} \overline{f(x)} g(x) d x\right) ; \text { and so } \\
\|f\|_{E}^{2} & =\frac{1}{2}\left(\int_{0}^{1}\left|f^{\prime}(x)\right|^{2} d x+\int_{0}^{1}|f(x)|^{2} d x\right), \forall f, g \in \mathscr{H}_{E} .
\end{aligned}
$$

Set

$$
\begin{aligned}
P(f, g) & =\int_{0}^{1} \overline{f_{n}(x)} g(x) d \beta(x), \text { where } \\
\beta & :=\frac{\delta_{0}+\delta_{1}}{2}, \text { i.e., Dirac masses at endpoints }
\end{aligned}
$$

where $g_{n}$ denotes the inward normal derivative at endpoints.

Let

$$
\mathscr{H}_{F}:=\left\{\begin{array}{c}
f \in C[0,1] \mid\|f\|_{E}^{2}+P_{2}(f)<\infty \text { where } \\
P_{2}(f):=P(f, f)=\int_{0}^{1}|f(x)|^{2} d \beta(x)
\end{array}\right\}
$$

Then we have following:

(1) As a Green-Gauss-Stoke principle, we have

$$
\begin{aligned}
\|\cdot\|_{F}^{2} & =\|\cdot\|_{E}^{2}+P_{2} \text { i.e. } \\
\|f\|_{F}^{2} & =\|f\|_{E}^{2}+\int_{0}^{1}|f(x)|^{2} d \beta(x) .
\end{aligned}
$$

(2) Moreover,

$$
\left\langle F_{x}, g\right\rangle_{E}=g(x)-\frac{e^{-x} g(0)+e^{-(1-x)} g(1)}{2} .
$$

(3) As a result of (4.3.38), eqs. (4.3.36)-(4.3.37) is the reproducing property in $\mathscr{H}_{F}$. Specifically, we have

$$
\left\langle F_{x}, g\right\rangle_{\mathscr{H}_{F}}=g(x), \forall g \in \mathscr{H}_{F} \text {. (see (4.3.35)) }
$$

Proof. Given $g \in \mathscr{H}_{F}$, one checks directly that

$$
\begin{aligned}
P\left(F_{x}, g\right) & =\int_{0}^{1} F_{x}(y) g(y) d \beta(y) \\
& =\frac{F_{x}(0) g(0)+F_{x}(1) g(1)}{2} \\
& =\frac{e^{-x} g(0)+e^{-(1-x)} g(1)}{2} ;
\end{aligned}
$$

and, using integration by parts, we have

$$
\begin{aligned}
\left\langle F_{x}, g\right\rangle_{E} & =g(x)-\frac{e^{-x} g(0)+e^{-(1-x)} g(1)}{2} \\
& =\left\langle F_{x}, g\right\rangle_{F}-P\left(F_{x}, g\right) .
\end{aligned}
$$




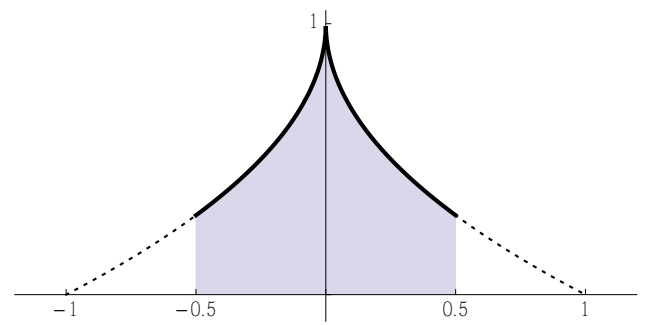

Figure 4.3.5. $F_{p}(x)=1-|x|^{p}, x \in\left(-\frac{1}{2}, \frac{1}{2}\right)$ and $0<p \leq 1$.

Now, using

$$
\int_{\partial \Omega}\left(F_{x}\right)_{n} g d \beta:=\frac{e^{-x} g(0)+e^{-(1-x)} g(1)}{2},
$$

and $\left\langle F_{x}, g\right\rangle_{\mathscr{H}_{F}}=g(x), \forall x \in(0,1)$, and using (4.3.38), the desired conclusion

$$
\left\langle F_{x}, g\right\rangle_{\mathscr{H}_{F}}=\underbrace{\left\langle F_{x}, g\right\rangle_{E}}_{\text {energy term }}+\int_{\partial \Omega}\left(F_{x}\right)_{n} g d \beta
$$

follows. Since the $\mathscr{H}_{F}$-norm closure of the span of $\left\{F_{x} \mid x \in(0,1)\right\}$ is all of $\mathscr{H}_{F}$, from (4.3.41), we further conclude that

$$
\langle f, g\rangle_{\mathscr{H}_{F}}=\langle f, g\rangle_{E}+\int_{\partial \Omega} \overline{f_{n}} g d \beta
$$

holds for all $f, g \in \mathscr{H}_{F}$.

In (4.3.41) and (4.3.42), we used $f_{n}$ to denote the inward normal derivative, i.e., $f_{n}(0)=f^{\prime}(0)$, and $f_{n}(1)=-f^{\prime}(1), \forall f \in \mathscr{H}_{E}$.

EXAMPLE 4.3.10. Fix $p, 0<p \leq 1$, and set

$$
F_{p}(x):=1-|x|^{p}, x \in\left(-\frac{1}{2}, \frac{1}{2}\right) ;
$$

see Figure 4.3.5. Then $F_{p}$ is positive definite and continuous; and so $-F_{p}^{\prime \prime}(x-y)$ is a p.d. kernel, so $-F_{p}^{\prime \prime}$ is a positive definite distribution on $\left(-\frac{1}{2}, \frac{1}{2}\right)$.

We saw that if $p=1$, then

$$
-F_{1}^{\prime \prime}=2 \delta
$$

where $\delta$ is the Dirac mass at $x=0$. But for $0<p<1,-F_{p}^{\prime \prime}$ does not have the form (4.3.44). We illustrate this if $p=\frac{1}{2}$. Then

$$
-F_{\frac{1}{2}}^{\prime \prime}=\chi_{\{x \neq 0\}} \frac{1}{4}|x|^{-\frac{3}{2}}+\frac{1}{4} \delta^{\prime \prime}
$$

where $\delta^{\prime \prime}$ is the double derivative of $\delta$ in the sense of distributions.

REMARK 4.3.11. There is a notion of boundary measure in potential theory. Boundary measures exist for any potential theory. In our case it works even more generally, whenever p.d. $F_{e x}$ for bounded domains $\Omega \subset \mathbb{R}^{n}$, and even Lie groups. But in the example of $F_{3}$, the boundary is two points.

In all cases, we get $\mathscr{H}_{F}$ as a perturbation of the energy form:

$$
\|\cdot\|_{\mathscr{H}_{F}}^{2}=\text { energy form }+ \text { perturbation }
$$

It is a Green-Gauss-Stoke principle. There is still a boundary measure for ALL bounded domains $\Omega \subset \mathbb{R}^{n}$, and even Lie groups. 
And RKHS form

$$
\|\cdot\|_{\mathscr{H}_{F}}^{2}=\text { energy form }+\int_{\partial \Omega} f_{n} f d \mu_{\text {bd meas. }}
$$

For the general theory of boundary measures and their connection to the Green-Gauss-Stoke principle, we refer readers to [JP13b, JP13c, Mov12, Bat90, Tel83, ACF09].

The approach via $\|\cdot\|_{\mathscr{H}_{F}}^{2}=$ "energy term + boundary term", does not fail to give a RKHS, but we must replace "energy term" with an abstract Dirichlet form; see Refs [HS12, Tre88].

4.3.4. Integral Kernels and Positive Definite Functions. Let $0<H<1$ be given, and set

$$
K_{H}(x, y)=\frac{1}{2}\left(|x|^{2 H}+|y|^{2 H}-|x-y|^{2 H}\right), \forall x, y \in \mathbb{R} .
$$

It is known that $K_{H}(\cdot, \cdot)$ is the covariance kernel for fractional Brownian motion [AJ12, AJL11, AL08, Aur11]. The special case $H=\frac{1}{2}$ is Brownian motion; and if $H=\frac{1}{2}$, then

See Figure 4.3.6.

$$
K_{\frac{1}{2}}(x, y)=|x| \wedge|y|=\min (|x|,|y|) .
$$

Set

$$
\begin{aligned}
\widetilde{F}_{H}(x, y) & :=1-|x-y|^{2 H} \\
F_{H}(x) & :=1-|x|^{2 H}
\end{aligned}
$$

and we recover $F(x)=1-|x|\left(=F_{2}\right)$ as a special case of $H=\frac{1}{2}$.
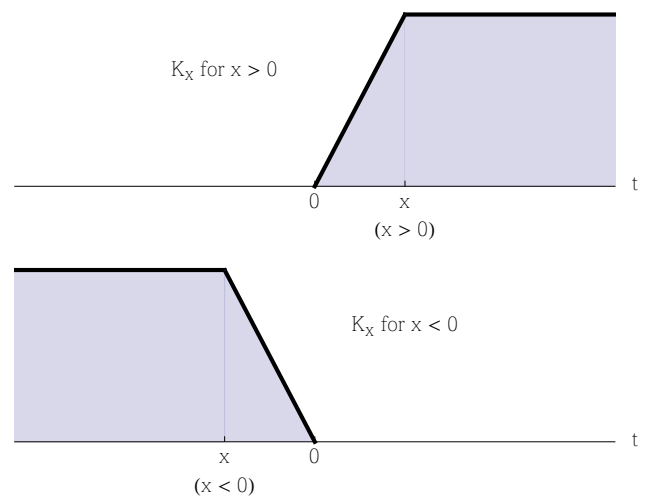

FIGURE 4.3.6. The integral kernel $K_{\frac{1}{2}}(x, y)=|x| \wedge|y|$.

Solving for $F_{H}$ in (4.3.48) $(0<H<1$ fixed), we then get

$$
F_{H}(x-y)=\underbrace{1-|x|^{2 H}-|y|^{2 H}}_{L_{H}}+2 K_{H}(x, y) ;
$$

and specializing to $x, y \in[0,1]$; we then get

$$
F(x-y)=\underbrace{1-x-y}_{L}+2(x \wedge y)
$$


or written differently:

$$
K_{F}(x, y)=L(x, y)+2 E(x, y)
$$

where $E(x, y)=x \wedge y$ is the familiar covariance kernel for Brownian motion.

Introducing the Mercer integral kernels corresponding to (4.3.49), we therefore get:

$$
\begin{aligned}
\left(T_{F_{H}} \varphi\right)(x) & =\int_{0}^{1} \varphi(y) F_{H}(x-y) d y \\
\left(T_{L_{H}} \varphi\right)(x) & =\int_{0}^{1} \varphi(y) L_{H}(x, y) d y \\
\left(T_{K_{H}} \varphi\right)(x) & =\int_{0}^{1} \varphi(y) K_{H}(x, y) d y
\end{aligned}
$$

for all $\varphi \in L^{2}(0,1)$, and all $x \in[0,1]$. Note the special case of (4.3.53) for $H=\frac{1}{2}$ is

$$
\left(T_{E} \varphi\right)(x)=\int_{0}^{1} \varphi(y)(x \wedge y) d y, \varphi \in L^{2}(0,1), x \in[0,1] .
$$

We have the following lemma for these Mercer operators:

Lemma 4.3.12. Let $0<H<1$, and let $F_{H}, L_{H}$ and $K_{H}$ be as in (4.3.49), then the corresponding Mercer operators satisfy:

$$
T_{F_{H}}=T_{L_{H}}+2 T_{K_{H}} .
$$

Proof. This is an easy computation, using (4.3.49), and (4.3.51)-(4.3.53).

4.3.5. Ornstein-Uhlenbeck. The reproducing kernel Hilbert space $\mathscr{H}_{F_{2}}$ in (4.3.7) is used in computations of Ito-integrals of Brownian motion; while the corresponding RKHS $\mathscr{H}_{F 3}$ from (4.3.27)-(4.3.28) is used in calculations of stochastic integration with the Ornstein-Uhlenbeck process.

Motivated by Newton's second law of motion, the Ornstein-Uhlenbeck velocity process is proposed to model a random external driving force. In $1 \mathrm{D}$, the process is the solution to the following stochastic differential equation

$$
d v_{t}=-\gamma v_{t} d t+\beta d B_{t}, \gamma, \beta>0 .
$$

Here, $-\gamma v_{t}$ is the dissipation, $\beta d B_{t}$ denotes a random fluctuation, and $B_{t}$ is the standard Brownian motion.

Assuming the particle starts at $t=0$. The solution to (4.3.55) is a Gaussian stochastic process such that

$$
\begin{aligned}
\mathbb{E}\left[v_{t}\right] & =v_{0} e^{-\gamma t} \\
\operatorname{var}\left[v_{t}\right] & =\frac{\beta^{2}}{2 \gamma}\left(1-e^{-2 \gamma t}\right) ;
\end{aligned}
$$

with $v_{0}$ being the initial velocity. See Figure 4.3.7. Moreover, the process has the following covariance function

$$
c(s, t)=\frac{\beta^{2}}{2 \gamma}\left(e^{-\gamma|t-s|}-e^{-\gamma|s+t|}\right) .
$$

If we wait long enough, it turns to a stationary process such that

$$
c(s, t) \sim \frac{\beta^{2}}{2 \gamma} e^{-\gamma|t-s|} .
$$




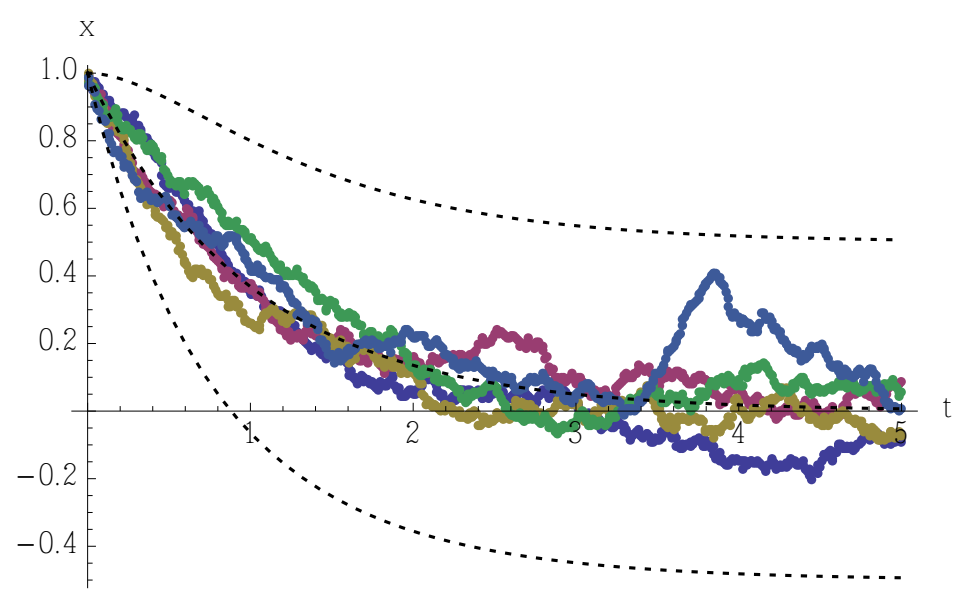

Figure 4.3.7. Monte-Carlo simulation of Ornstein-Uhlenbeck process with 5 sample paths. $\beta=\gamma=1, v_{0}=1, t \in[0,5]$

This corresponds to the function $F_{3}$.

4.3.6. An Overview of the Two Cases: $F_{2}$ and $F_{3}$. In Table 4.4.1 below, we give a list of properties for the particular two cases $F_{2}$ and $F_{3}$ from table 3.1.1. Special attention to these examples is merited; first they share a number of properties with much wider families of locally defined positive definite functions; and these properties are more transparent in their simplest form. Secondly, there are important differences between cases $F_{2}$ and $F_{3}$, and the table serves to highlight both differences and similarities. A particular feature that is common for the two is that, when the Mercer operator $T_{F}$ is introduced, then its inverse $T_{F}^{-1}$ exists as an unbounded positive and selfadjoint operator in $\mathscr{H}_{F}$. Moreover, in each case, this operator coincides with the Friedrichs extension of a certain second order Hermitian semibounded operator (calculated from $D^{(F)}$ ), with dense domain in $\mathscr{H}_{F}$.

\subsection{Higher Dimensions}

Our function above for $F_{3}$ (sect. 4.3.1 and 4.3.3) admits a natural extension to $\mathbb{R}^{n}, n>1$, as follows.

Let $\Omega \subset \mathbb{R}^{n}$ be a subset satisfying $\Omega \neq \phi, \Omega$ open and connected; and assume $\bar{\Omega}$ is compact. Let $\triangle=\sum_{i=1}^{n}\left(\partial / \partial x_{i}\right)^{2}$ be the usual Laplacian in $n$-variables.

Lemma 4.4.1. Let $F: \Omega-\Omega \rightarrow \mathbb{C}$ be continuous and positive definite; and let $\mathscr{H}_{F}$ be the corresponding RKHS. In $\mathscr{H}_{F}$, we set

$$
\begin{aligned}
L^{(F)}\left(F_{\varphi}\right) & :=F_{\triangle \varphi}, \forall \varphi \in C_{c}^{\infty}(\Omega), \text { with } \\
\operatorname{dom}\left(L^{(F)}\right) & =\left\{F_{\varphi} \mid \varphi \in C_{c}^{\infty}(\Omega)\right\} ;
\end{aligned}
$$

then $L^{(F)}$ is semibounded in $\mathscr{H}_{F}, L^{(F)} \leq 0$ in the order of Hermitian operators.

Let $T_{F}$ be the Mercer operator; then there is a positive constant $c\left(=c_{(F, \Omega)}>0\right)$ such that $T_{F}^{-1}$ is a selfadjoint extension of the densely defined operator $c\left(I-L^{(F)}\right)$ in $\mathscr{H}_{F}$. 
1. $F_{2}(x)=1-|x|,|x|<\frac{1}{2}$

2. Mercer operator

$$
T_{F_{2}}: L^{2}\left(0, \frac{1}{2}\right) \rightarrow L^{2}\left(0, \frac{1}{2}\right)
$$

3. $T_{F_{2}}^{-1}$ is unbounded, selfadjoint

Proof. Since $T_{F}$ is positive, bounded, and trace class, it follows that $T_{F_{2}}^{-1}$ is positive, unbounded, and selfadjoint.

4. $T_{F_{2}}^{-1}=$ Friedrichs extension of

$$
-\left.\frac{1}{2}\left(\frac{d}{d x}\right)^{2}\right|_{C_{c}^{\infty}\left(0, \frac{1}{2}\right)}
$$

as a selfadjoint operator on $L^{2}\left(0, \frac{1}{2}\right)$. Sketch of proof.

Setting

$$
u(x)=\int_{0}^{\frac{1}{2}} \varphi(y)(1-|x-y|) d y
$$

then

$$
\begin{aligned}
u^{\prime \prime} & =-2 \varphi \\
& \mathbb{} \\
u & =\left(-\frac{1}{2}\left(\frac{d}{d x}\right)^{2}\right)^{-1} \varphi
\end{aligned}
$$

and so

$$
T_{F_{2}}=\left(-\frac{1}{2}\left(\frac{d}{d x}\right)^{2}\right)^{-1} .
$$

And we get a selfadjoint extension

$$
T_{F_{2}}^{-1} \supset-\frac{1}{2}\left(\frac{d}{d x}\right)^{2}
$$

in $L^{2}\left(0, \frac{1}{2}\right)$, where the containment refers to operator graphs.
1. $F_{3}(x)=e^{-|x|},|x|<1$

2. Mercer operator

$$
T_{F_{3}}: L^{2}(0,1) \rightarrow L^{2}(0,1)
$$

3. $T_{F_{3}}^{-1}$ is unbounded, selfadjoint

Proof. Same argument as in the proof for $T_{F_{2}}^{-1}$; also follows from Mercer's theorem.

4. $T_{F_{2}}^{-1}=$ Friedrichs extension of

$$
\left.\frac{1}{2}\left(I-\left(\frac{d}{d x}\right)^{2}\right)\right|_{C_{c}^{\infty}(0,1)}
$$

as a selfadjoint operator on $L^{2}(0,1)$.

Sketch of proof.

Setting

$$
u(x)=\int_{0}^{1} \varphi(y) e^{-|x-y|} d y
$$

then

$$
\begin{aligned}
u^{\prime \prime} & =u-2 \varphi \\
& \mathbb{} \\
u & =\left(\frac{1}{2}\left(1-\left(\frac{d}{d x}\right)^{2}\right)\right)^{-1} \varphi
\end{aligned}
$$

and so

$$
T_{F_{3}}=\left(\frac{1}{2}\left(I-\left(\frac{d}{d x}\right)^{2}\right)\right)^{-1} .
$$

Now, a selfadjoint extension

$$
T_{F_{3}}^{-1} \supset \frac{1}{2}\left(I-\left(\frac{d}{d x}\right)^{2}\right)
$$

in $L^{2}(0,1)$.

TABLE 4.4.1. An overview of two cases: $F_{2}$ v.s. $F_{3}$.

PRoOF. The ideas for the proof are contained in section 4.3.1 and 4.3.3 above. To get the general conclusions, we may combine these considerations with the general theory of Green's functions for elliptic linear PDEs (partial differential equations); see also [Nel57, JLW69]. 


\section{CHAPTER 5}

\section{Comparing Different RKHSs $\mathscr{H}_{F}$ and $\mathscr{H}_{K}$}

Before we turn to comparison of pairs of RKHSs, we will prove a lemma which accounts for two uniformity principles for positive definite continuous functions defined on open subsets in locally compact groups:

LEMmA 5.0.2. Let $G$ be a locally compact group, and let $\Omega \subset G$ be an open and connected subset, $\Omega \neq \phi$. Let $F: \Omega^{-1} \Omega \rightarrow \mathbb{C}$ be a continuous positive definite function satisfying $F(e)=1$, where $e \in G$ is the unit for the group operation.

(1) Then $F$ extends by limit to a continuous p.d. function

$$
F^{(e x)}: \bar{\Omega}^{-1} \bar{\Omega} \longrightarrow \mathbb{C} \text {. }
$$

(2) Moreover, the two p.d. functions $F$ and $F^{(e x)}$ have the same RKHS consisting of continuous functions $\xi$ on $\bar{\Omega}$ such that, $\exists 0<A<\infty, A=A_{\xi}$, s.t.

$$
\left|\int_{\bar{\Omega}} \overline{\xi(x)} \varphi(x) d x\right|^{2} \leq A\left\|F_{\varphi}\right\|_{\mathscr{H}_{F}}^{2}, \forall \varphi \in C_{c}(\Omega)
$$

where $d x=$ Haar measure on $G$,

$$
F_{\varphi}(\cdot)=\int_{\Omega} \varphi(y) F\left(y^{-1} \cdot\right) d y, \varphi \in C_{c}(\Omega)
$$

and $\left\|F_{\varphi}\right\|_{\mathscr{H}_{F}}$ denotes the $\mathscr{H}_{F}$-norm of $F_{\varphi}$.

(3) Every $\xi \in \mathscr{H}_{F}$ satisfies the following a priori estimate:

$$
|\xi(x)-\xi(y)|^{2} \leq 2\|\xi\|_{\mathscr{H}_{F}}^{2}\left(1-\Re\left\{F\left(x^{-1} y\right)\right\}\right)
$$

for all $\xi \in \mathscr{H}_{F}$, and all $x, y \in \bar{\Omega}$.

PRoOF. The arguments in the proof only use standard tools from the theory of reproducing kernel Hilbert spaces. We covered a special case in section 4.1, we shall omit the details here.

Now add the further assumption on the subset $\Omega \subset G$ from the lemma: Assume in addition that $\Omega$ has compact closure, so $\bar{\Omega}$ is compact. Let $\partial \Omega=\bar{\Omega} \backslash \Omega$ be the boundary. With this assumption, we get automatically the inclusion

$$
C(\bar{\Omega}) \subset L^{2}(\bar{\Omega})
$$

since continuous functions on $\bar{\Omega}$ are automatically uniformly bounded, and Haar measure on $G$ has the property that $|\bar{\Omega}|<\infty$.

Definition 5.0.3. Let $(\Omega, F)$ be as above, i.e.,

$$
F: \bar{\Omega}^{-1} \bar{\Omega} \longrightarrow \mathbb{C}
$$


is continuous and positive definite. Assume $G$ is a Lie group. Recall, extension by limit to $\bar{\Omega}$ is automatic by the limit. Let $\beta \in \mathscr{M}(\partial \Omega)$ be a positive finite measure on the boundary $\partial \Omega$. We say that $\beta$ is a boundary measure iff

$$
\left\langle T_{F} f, \xi\right\rangle_{\mathscr{H}_{F}}-\int_{\Omega} \overline{f(x)} \xi(x) d x=\int_{\partial \Omega} \overline{\left(T_{F} f\right)_{n}(\sigma)} \xi(\sigma) d \beta(\sigma)
$$

holds for all $f \in C(\bar{\Omega}), \forall \xi \in \mathscr{H}_{F}$, where $(\cdot)_{n}=$ normal derivative computed on the boundary $\partial \Omega$ of $\Omega$.

Remark 5.0.4. For the example $G=\mathbb{R}, \bar{\Omega}=[0,1], \partial \Omega=\{0,1\}$, and $F=F_{3}$ on $[0,1]$, where

$$
F(x)=e^{-|x|}, \forall x \in[-1,1]
$$

the boundary measure is

$$
\beta=\frac{1}{2}\left(\delta_{0}+\delta_{1}\right)
$$

Let $G$ be a locally compact group with left-Haar measure, and let $\Omega \subset G$ be a non-empty subset satisfying: $\Omega$ is open and connected; and is of finite Haar measure; write $|\Omega|<\infty$. The Hilbert space $L^{2}(\Omega)=L^{2}(\Omega, d x)$ is the usual $L^{2}$ space of measurable functions $f$ on $\Omega$ such that

$$
\|f\|_{L^{2}(\Omega)}^{2}:=\int_{\Omega}|f(x)|^{2} d x<\infty .
$$

Definition 5.0.5. Let $F$ and $K$ be two continuous and positive definite functions defined on

$$
\Omega^{-1} \cdot \Omega:=\left\{x^{-1} y \mid x, y \in \Omega\right\} .
$$

We say that $K \ll F$ iff there is a finite constant $A$ such that for all finite subsets $\left\{x_{i}\right\}_{i=1}^{N} \subset \Omega$, and all systems $\left\{c_{i}\right\}_{i=1}^{N} \subset \mathbb{C}$, we have:

$$
\sum_{i} \sum_{j} \overline{c_{i}} c_{j} K\left(x_{i}^{-1} x_{j}\right) \leq A \sum_{i} \sum_{j} \overline{c_{i}} c_{j} F\left(x_{i}^{-1} x_{j}\right) .
$$

Lemma 5.0.6. Let $F$ and $K$ be as above; then $K \ll F$ if and only if there is a finite constant $A \in \mathbb{R}_{+}$such that

$$
\int_{\Omega} \int_{\Omega} \overline{\varphi(x)} \varphi(y) K\left(x^{-1} y\right) d x d y \leq A \int_{\Omega} \int_{\Omega} \overline{\varphi(x)} \varphi(y) F\left(x^{-1} y\right) d x d y
$$

holds for all $\varphi \in C_{c}(\Omega)$. The constant $A$ in (5.0.10) and (5.0.11) will be the same.

Proof. Easy; use an approximate identity in $G$, see e.g., [Rud73, Rud90].

Setting

$$
F_{\varphi}(x)=\int_{\Omega} \varphi(y) F\left(y^{-1} x\right) d y,
$$

and similarly for $K_{\varphi}=\int_{\Omega} \varphi(y) K\left(y^{-1} \cdot\right) d y$, we note that $K \ll F$ if and only if:

$$
\left\|K_{\varphi}\right\|_{\mathscr{H}_{K}} \leq \sqrt{A}\left\|F_{\varphi}\right\|_{\mathscr{H}_{F}}, \forall \varphi \in C_{c}(\Omega) .
$$

Further, note that, if $G$ is also a Lie group, then (5.0.13) follows from checking it only for all $\varphi \in C_{c}^{\infty}(G)$. See Lemma 2.2.7. 
Theorem 5.0.7. Let $\Omega, F$ and $K$ be as in Definition 5.0.5, i.e., both continuous and p.d. on the set $\Omega^{-1} \cdot \Omega$ in (5.0.9); then the following two conditions are equivalent:

(i) $K \ll F$, and

(ii) $\mathscr{H}_{K}$ is a closed subspace of $\mathscr{H}_{F}$.

Proof. $\Downarrow$ Assume $K \ll F$, we then define a linear operator $l: \mathscr{H}_{F} \rightarrow \mathscr{H}_{K}$, setting

$$
l\left(F_{\varphi}\right):=K_{\varphi}, \forall \varphi \in C_{c}(\Omega) .
$$

We now use (5.0.13), plus the fact that $\mathscr{H}_{F}$ is the $\|\cdot\|_{\mathscr{H}_{F}}$-completion of

$$
\left\{F_{\varphi} \mid \varphi \in C_{c}(\Omega)\right\}
$$

and similarly for $\mathscr{H}_{K}$.

As a result of (5.0.14) and (5.0.13), we get a canonical extension of $l$ to a bounded linear operator, also denoted $l: \mathscr{H}_{F} \rightarrow \mathscr{H}_{K}$, and

$$
\|l(\xi)\|_{\mathscr{H}_{K}} \leq \sqrt{A}\|\xi\|_{\mathscr{H}_{F}} \text {, for all } \xi \in \mathscr{H}_{K} \text {. }
$$

We interrupt the proof to give a lemma:

Lemma 5.0.8. Let $F, K, \Omega$ be as above. Assume $K \ll F$, and let $l: \mathscr{H}_{F} \rightarrow$ $\mathscr{H}_{K}$ be the bounded operator introduced in (5.0.14) and (5.0.15). Then the adjoint operator $l^{*}: \mathscr{H}_{K} \rightarrow \mathscr{H}_{F}$ satisfies:

$$
\left(l^{*}(\xi)\right)(x)=\xi(x), \text { for all } \xi \in \mathscr{H}_{K}, x \in \Omega .
$$

Proof. By the definite of $l^{*}$, as the adjoint of a bounded linear operator between Hilbert spaces, we get

$$
\left\|l^{*}\right\|_{\mathscr{H}_{K} \rightarrow \mathscr{H}_{F}}=\|l\|_{\mathscr{H}_{F} \rightarrow \mathscr{H}_{K}} \leq \sqrt{A}
$$

for the respective operator norms; and

$$
\left\langle l^{*}(\xi), F_{\varphi}\right\rangle_{\mathscr{H}_{F}}=\left\langle\xi, K_{\varphi}\right\rangle_{\mathscr{H}_{K}}, \forall \varphi \in C_{c}(\Omega) .
$$

Using now the reproducing property in the two RKHSs, we get:

$$
\begin{aligned}
(\mathrm{LHS})_{(5.0 .18)} & =\int_{\Omega} \overline{l^{*}(\xi)(x)} \varphi(x) d x, \text { and } \\
(\mathrm{RHS})_{(5.0 .18)} & =\int_{\Omega} \overline{\xi(x)} \varphi(x) d x, \text { for all } \varphi \in C_{c}(\Omega) .
\end{aligned}
$$

Taking now approximations in $C_{c}(\Omega)$ to the Dirac masses $\left\{\delta_{x} \mid x \in \Omega\right\}$, the desired conclusion (5.0.16) follows.

Proof of Theorem 5.0.7 Resumed . Assume $K \ll F$, the lemma proves that $\mathscr{H}_{K}$ identifies with a linear space of continuous functions $\xi$ on $\Omega$, and if $\xi \in \mathscr{H}_{K}$, then it is also in $\mathscr{H}_{F}$.

We claim that this is a closed subspace in $\mathscr{H}_{F}$ relative to the $\mathscr{H}_{F}$-norm.

Step 1. Let $\left\{\xi_{n}\right\} \subset \mathscr{H}_{K}$ satisfying

$$
\lim _{n, m \rightarrow \infty}\left\|\xi_{n}-\xi_{m}\right\|_{\mathscr{H}_{F}}=0 .
$$

By (5.0.15) and (5.0.16), the lemma; we get

$$
\lim _{n, m \rightarrow \infty}\left\|\xi_{n}-\xi_{m}\right\|_{\mathscr{H}_{K}}=0 .
$$


$\underline{\text { Step 2. }}$. Since $\mathscr{H}_{K}$ is complete, we get $\chi \in \mathscr{H}_{K}$ such that

$$
\lim _{n \rightarrow \infty}\left\|\xi_{n}-\chi\right\|_{\mathscr{H}_{K}}=0 \text {. }
$$

Step 3. We claim that this $\mathscr{H}_{K}$-limit $\chi$ also defines a unique element in $\mathscr{H}_{F}$, and it is therefore the $\mathscr{H}_{F}$-limit.

We have for all $\varphi \in C_{c}(\Omega)$ :

$$
\begin{aligned}
\left|\int_{\Omega} \overline{\chi(x)} \varphi(x) d x\right| & \leq\|\chi\|_{\mathscr{H}_{K}}\left\|K_{\varphi}\right\|_{\mathscr{H}_{K}} \\
& \leq\|\chi\|_{\mathscr{H}_{K}} \sqrt{A}\left\|F_{\varphi}\right\|_{\mathscr{H}_{F}}
\end{aligned}
$$

and so $\chi \in \mathscr{H}_{F}$.

We now turn to the converse implication of Theorem 5.0.7:

$\Uparrow$ Assume $F$ and $K$ are as in the statement of the theorem; and that $\mathscr{H}_{K}$ is a close subspace in $\mathscr{H}_{F}$ via identification of the respective continuous functions on $\Omega$. We then prove that $K \ll F$.

Now let $P_{K}$ denote the orthogonal projection of $\mathscr{H}_{F}$ onto the closed subspace $\mathscr{H}_{K}$. We claim that

$$
P_{K}\left(F_{\varphi}\right)=K_{\varphi}, \forall \varphi \in C_{c}(\Omega) .
$$

Using the uniqueness of the projection $P_{K}$, we need to verify that $F_{\varphi}-K_{\varphi} \in$ $\mathscr{H}_{F} \ominus \mathscr{H}_{K}$; i.e., that

$$
\left\langle F_{\varphi}-K_{\varphi}, \xi_{K}\right\rangle_{\mathscr{H}_{F}}=0 \text {, for all } \xi_{K} \in \mathscr{H}_{K}
$$

But since $\mathscr{H}_{K} \subset \mathscr{H}_{F}$, we have

$$
\operatorname{LHS}_{(5.0 .21)}=\int_{\Omega} \overline{\varphi(x)} \xi_{K}(x) d x-\int_{\Omega} \overline{\varphi(x)} \xi_{K}(x) d x=0,
$$

for all $\varphi \in C_{c}(\Omega)$. This proves (5.0.20).

To verify $K \ll F$, we use the criterion (5.0.13) from Lemma 5.0.6. Indeed, consider $K_{\varphi} \in \mathscr{H}_{K}$. Since $\mathscr{H}_{K} \subset \mathscr{H}_{F}$, we get

$$
\begin{gathered}
l\left(F_{\varphi}\right)=P_{K}\left(F_{\varphi}\right)=K_{\varphi}, \text { and } \\
\left\|K_{\varphi}\right\|_{\mathscr{H}_{K}}=\left\|l\left(F_{\varphi}\right)\right\|_{\mathscr{H}_{K}} \leq \sqrt{A}\left\|F_{\varphi}\right\|_{\mathscr{H}_{F}}
\end{gathered}
$$

which is the desired estimate (5.0.13).

\subsection{Applications}

Below we give an application of Theorem 5.0.7 to the deficiency-index problem, and to the computation of the deficiency spaces; see also Lemma 2.10.4, and Lemma 7.2.1.

As above, we will consider two given continuous p.d. functions $F$ and $K$, but the group now is $G=\mathbb{R}$ : We pick $a, b \in \mathbb{R}_{+}, 0<a<b$, such that $F$ is defined on $(-b, b)$, and $K$ on $(-a, a)$. The corresponding two RKHSs will be denoted $\mathscr{H}_{F}$ and $\mathscr{H}_{K}$. We say that $K \ll F$ iff there is a finite constant $A$ such that

$$
\left\|K_{\varphi}\right\|_{\mathscr{H}_{K}}^{2} \leq A\left\|F_{\varphi}\right\|_{\mathscr{H}_{F}}^{2}
$$

for all $\varphi \in C_{c}(0, a)$. Now this is a slight adaptation of our Definition 5.0.5 above, but this modification will be needed; for example in computing the indices of two 
p.d. functions $F_{2}$ and $F_{3}$ from Table 3.1 .1 ; see also section 4.3 below. In fact, a simple direct checking shows that

$$
F_{2} \ll F_{3} \quad \text { (see Table 3.1.1), }
$$

and we now take $a=\frac{1}{2}, b=1$.

Here, $F_{2}(x)=1-|x|$ in $|x|<\frac{1}{2}$; and $F_{3}(x)=e^{-|x|}$ in $|x|<1$; see Figure 5.1.1.
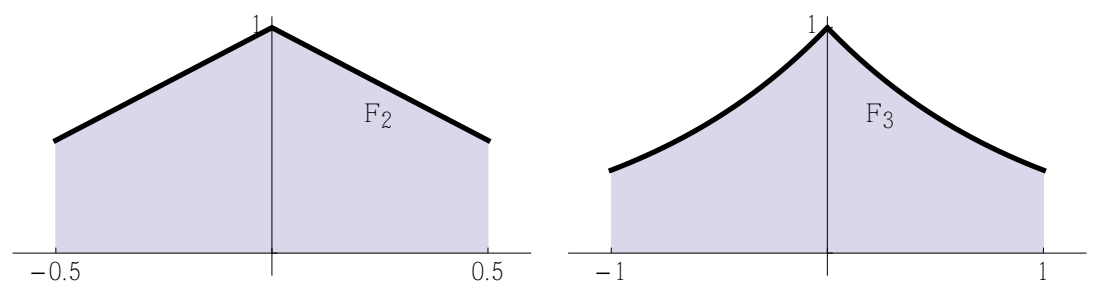

Figure 5.1.1. The examples of $F_{2}$ and $F_{3}$.

We wish to compare the respective skew-Hermitian operators, $D^{(F)}$ in $\mathscr{H}_{F}$; and $D^{(F)}$ in $\mathscr{H}_{K}$; see section 2.5, i.e.,

$$
\begin{aligned}
D^{(F)}\left(F_{\varphi}\right) & =F_{\varphi^{\prime}}, \forall \varphi \in C_{c}^{\infty}(0, b) ; \text { and } \\
D^{(K)}\left(K_{\varphi}\right) & =K_{\varphi^{\prime}}, \forall \varphi \in C_{c}^{\infty}(0, a) .
\end{aligned}
$$

Let $z \in \mathbb{C}$; and we set

$$
\begin{aligned}
& D E F_{F}(z)=\left\{\xi \in \operatorname{dom}\left(\left(D^{(F)}\right)^{*}\right) \mid\left(D^{(F)}\right)^{*} \xi=z \xi\right\}, \text { and } \\
& D E F_{K}(z)=\left\{\xi \in \operatorname{dom}\left(\left(D^{(K)}\right)^{*}\right) \mid\left(D^{(K)}\right)^{*} \xi=z \xi\right\} .
\end{aligned}
$$

TheOREm 5.1.1. Let two continuous p.d. functions $F$ and $K$ be specified as above, and suppose

$$
K \ll F
$$

then

$$
D E F_{K}(z)=\left.D E F_{F}(z)\right|_{(0, a)}
$$

i.e., restriction to the smaller interval.

Proof. Since (5.1.7) is assumed, it follows from Theorem 5.0.7, that $\mathscr{H}_{K}$ is a subspace of $\mathscr{H}_{F}$.

If $\varphi \in C_{c}^{\infty}(0, b)$, and $\xi \in \operatorname{dom}\left(\left(D^{(F)}\right)^{*}\right)$, then

$$
\left\langle\left(D^{(F)}\right)^{*} \xi, F_{\varphi}\right\rangle_{\mathscr{H}_{F}}=\left\langle\xi, F_{\varphi^{\prime}}\right\rangle_{\mathscr{H}_{F}}=\int_{0}^{b} \overline{\xi(x)} \varphi^{\prime}(x) d x ;
$$

and it follows that functions $\xi$ in $D E F_{F}(z)$ must be multiples of

$$
(0, b) \ni x \longmapsto e_{z}(x)=e^{-z x} \text {. }
$$

Hence, by Theorem 5.0.7, we get

$$
D E F_{K}(z) \subseteq D E F_{F}(z),
$$

and by (5.1.9), we see that(5.1.8) must hold.

Conversely, if $D E F_{F}(z) \neq 0$, then $l\left(D E F_{F}(z)\right) \neq 0$, and its restriction to $(0, a)$ is contained in $D E F_{K}(z)$. The conclusion in the theorem follows. 
Remark 5.1.2. The spaces $D E F_{(F)}(z), z \in \mathbb{C}$, are also discussed in Theorems 2.10.9-2.10.10.

Example 5.1.3 (Application). Consider the two functions $F_{2}$ and $F_{3}$ in Table 3.1.1. Both of the operators $D^{\left(F_{i}\right)}, i=2,3$, have deficiency indices $(1,1)$.

Proof. One easily checks that $F_{2} \ll F_{3}$. And it is also easy to check directly that $D^{\left(F_{2}\right)}$ has indices $(1,1)$. Hence, by $(5.1 .8)$ in the theorem, it follows that $D^{\left(F_{3}\right)}$ also must have indices $(1,1)$. (The latter conclusion is not as easy to verify by direct means!)

\subsection{Radially Symmetric Positive Definite Functions}

Among other subclasses of positive definite functions we have radially symmetric p.d. functions. If a given p.d. function happens to be radially symmetric, then there are a number of simplifications available, and the analysis in higher dimension often simplifies. This is to a large extend due to theorems of I. J. Schöenberg and D. V. Widder. Below we sketch two highpoints, but we omit details and application to interpolation and to geometry. These themes are in the literature, see e.g. [Sch38, SW53, Sch64, Wid41, WW75].

REMARK 5.2.1. In some cases, the analysis in one dimension yields insight into the possibilities in $\mathbb{R}^{k}, k>1$. This leads for example for functions $F$ on $\mathbb{R}^{k}$ which are radial, i.e., of the form $F(x)=\Phi\left(\|x\|^{2}\right)$, where $\|x\|^{2}=\sum_{i=1}^{k} x_{i}^{2}$.

A function $q$ on $\mathbb{R}_{+}, q: \mathbb{R}_{+} \rightarrow \mathbb{R}$, is said to be completely monotone iff $q \in$ $C([0, \infty)) \cap C^{\infty}((0, \infty))$ and

$$
(-1)^{n} q^{(n)}(r) \geq 0, r \in \mathbb{R}_{+}, n \in \mathbb{N}_{0} .
$$

EXAMPLE 5.2.2.

$$
\begin{aligned}
& q(r)=e^{-\alpha r}, \quad \alpha \geq 0 ; \\
& q(r)=\frac{\alpha}{r^{1-\alpha}}, \quad \alpha \leq 1 ; \\
& q(r)=\frac{1}{\left(r+\alpha^{2}\right)^{\beta}}, \quad \alpha>0, \beta \geq 0 .
\end{aligned}
$$

TheOREM 5.2.3 (Schöenberg (1938)). A function $q: \mathbb{R}_{+} \rightarrow \mathbb{R}$ is completely monotone iff the corresponding function $F_{q}(x)=q\left(\|x\|^{2}\right)$ is positive definite and radial on $\mathbb{R}^{k}$ for all $k \in \mathbb{N}$.

Proof. We omit details, but the proof uses:

Theorem 5.2.4 (Bernstein-Widder). A function $q: \mathbb{R}_{+} \rightarrow \mathbb{R}$ is completely monotone iff there is a finite positive Borel measure on $\mathbb{R}_{+}$s.t.

$$
q(r)=\int_{0}^{\infty} e^{-r t} d \mu(t), r \in \mathbb{R}_{+},
$$

i.e., $q$ is the Laplace transform of a finite positive measure $\mu$ on $\mathbb{R}_{+}$.

REMARK 5.2.5. The condition that the function $q$ in (5.2.1) be in $C^{\infty}\left(\mathbb{R}_{+}\right)$ may be relaxed; and then (5.2.1) takes the following alternative form:

$$
\sum_{k=1}^{n}(-1)^{k}\left(\begin{array}{l}
n \\
k
\end{array}\right) q(r+k \delta) \geq 0
$$


for all $n \in \mathbb{N}$, all $\delta>0$, and $x \in[0, \infty)$; i.e.,

$$
\begin{gathered}
q(r)-q(r+\delta) \geq 0 \\
q(r)-2 q(r+\delta)+q(r+2 \delta) \geq 0 \text { e.t.c. }
\end{gathered}
$$

It is immediate that every completely monotone function $q$ on $[0, \infty)$ is convex.

\subsection{Connecting $F$ and $\bar{F}$ When $F$ is a Positive Definite Function}

Let $F:(-1,1) \rightarrow \mathbb{C}$ be continuous and positive definite, and let $\bar{F}$ be the complex conjugate, i.e., $\bar{F}(x)=F(-x), \forall x \in(-1,1)$. Below, we construct a contractive-linking operator $\mathscr{H}_{F} \rightarrow \mathscr{H}_{F}$ between the two RKHSs.

Lemma 5.3.1. Let $\mu$ and $\mu^{(s)}$ be as before, $\mu^{(s)}=\mu \circ s, s(x)=-x$; and set

$$
g=\sqrt{\frac{d \mu^{(s)}}{d \mu}} ;
$$

(the square root of the Radon-Nikodym derivative) then the g-multiplication operator is isometric between the respective Hilbert spaces; $L^{2}\left(\mu^{(s)}\right)$ and $L^{2}(\mu)$ as follows:

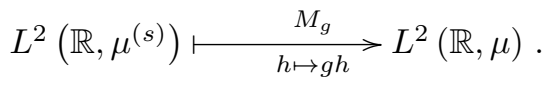

Proof. Let $h \in L^{2}\left(\mu^{(s)}\right)$, then

$$
\begin{aligned}
\int_{\mathbb{R}}|g h|^{2} d \mu & =\int_{\mathbb{R}}|h|^{2} \frac{d \mu^{(s)}}{d \mu} d \mu \\
& =\int_{\mathbb{R}}|h|^{2} d \mu^{(s)}=\|h\|_{L^{2}\left(\mu^{(s)}\right)}^{2} .
\end{aligned}
$$

Lemma 5.3.2. If $F:(-1,1) \rightarrow \mathbb{C}$ is a given continuous p.d. function, and if $\mu \in \operatorname{Ext}(F)$, then

$$
\mathscr{H}_{F} \ni F_{\varphi} \longmapsto V^{(F)} \longrightarrow \widehat{\varphi} \in L^{2}(\mathbb{R}, \mu)
$$

extends by closure to an isometry.

Proof. For $\varphi \in C_{c}(0,1)$, we have:

$$
\begin{aligned}
\left\|F_{\varphi}\right\|_{\mathscr{H}_{F}}^{2} & =\quad \int_{0}^{1} \int_{0}^{1} \overline{\varphi(x)} \varphi(y) F(x-y) d x d y \\
& =\int_{\mu \in \operatorname{Ext}(\mathrm{F})}^{1} \int_{0}^{1} \overline{\varphi(x)} \varphi(y)\left(\int_{\mathbb{R}} e^{i(x-y) \lambda} d \mu(\lambda)\right) d x d y \\
& =\int_{(\text {Fubini })}|\widehat{\varphi}(\lambda)|^{2} d \mu(\lambda) \\
& = \\
(5.3 .3) & \left\|V^{(F)}\left(F_{\varphi}\right)\right\|_{L^{2}(\mathbb{R}, \mu)}^{2}
\end{aligned}
$$


Definition 5.3.3. Set

$$
\begin{aligned}
\left(\varphi * g^{\vee}\right)(x) & :=\int_{0}^{1} \varphi(x) g^{\vee}(x-y) d y \\
& =\left(g^{\vee} * \varphi\right)(x), x \in(0,1), \varphi \in C_{c}(0,1) .
\end{aligned}
$$

Theorem 5.3.4. We have

$$
\left(V^{(F) *} M_{g} V^{(\bar{F})}\right)\left(\bar{F}_{\varphi}\right)=T_{F}\left(g^{\vee} * \varphi\right), \forall \varphi \in C_{c}(0,1) .
$$

Proof. Let $\varphi \in C_{c}(0,1)$, we will then compute the two sides in (5.3.5), where $g^{\vee}:=$ inverse Fourier transform:

$$
\operatorname{LHS}_{(5.3 .5)}=V^{(F) *}(g \widehat{\varphi}) ;
$$

$\left(\widehat{\varphi} \in L^{2}\left(\mu^{(s)}\right)\right.$, and using that $g \widehat{\varphi} \in L^{2}(\mu)$ by lemma 5.3.1) we get:

$$
\begin{aligned}
\operatorname{LHS}_{(5.3 .5)} & =\left(V^{(F)}\right)^{*}(\underbrace{\widehat{g^{\vee} * \varphi}}_{\in L^{2}(\mu)}) \\
& =T_{F}\left(g^{\vee} * \varphi\right)
\end{aligned}
$$

where $T_{F}$ is the Mercer operator $T_{F}: L^{2}(\Omega) \rightarrow \mathscr{H}_{F}$ defined using

$$
\begin{aligned}
T_{F}(\varphi)(x) & =\int_{0}^{1} \varphi(x) F(x-y) d y \\
& =\chi_{[0,1]}(x)(\widehat{\varphi} d \mu)^{\vee}(x) .
\end{aligned}
$$

COROLlary 5.3.5. Let $\mu$ and $\mu^{(s)}$ be as above, with $\mu \in \operatorname{Ext}(F)$, and $\mu^{(s)} \ll \mu$. Setting $g=\sqrt{\frac{d \mu^{(s)}}{d \mu}}$, we get

$$
\left\|\left(V^{(F)}\right)^{*} M_{g} V^{(\bar{F})}\right\|_{\mathscr{H}_{F} \rightarrow \mathscr{H}_{F}} \leq 1 .
$$

Proof. For the three factors in the composite operator $\left(V^{(F)}\right)^{*} M_{b} V^{(\bar{F})}$ in (5.3.6), we have two isometries as follows:

$$
\begin{aligned}
V^{(\bar{F})}: \mathscr{H}_{\bar{F}} & \rightarrow L^{2}\left(\mu^{(s)}\right), \text { and } \\
M_{g}: L^{2}\left(\mu^{(s)}\right) & \rightarrow L^{2}(\mu),
\end{aligned}
$$

and both isometries; while

$$
\left(V^{(F)}\right)^{*}: L^{2}(\mu) \rightarrow \mathscr{H}_{F}
$$

is co-isometric, and therefore contractive, i.e.,

$$
\left\|\left(V^{(F)}\right)^{*}\right\|_{L^{2}(\mu) \rightarrow \mathscr{H}_{F}} \leq 1
$$

But then:

$$
\begin{aligned}
\left\|\left(V^{(F)}\right)^{*} M_{g} V^{(\bar{F})}\right\|_{\mathscr{H}_{F} \rightarrow \mathscr{H}_{F}} & \leq\left\|\left(V^{(F)}\right)^{*}\right\|\left\|M_{g}\right\|\left\|V^{(\bar{F})}\right\| \\
& =\left\|\left(V^{(F)}\right)^{*}\right\| \leq 1 \text {, by }(5.3 .7) .
\end{aligned}
$$




\subsection{The Imaginary Part of a Positive Definite Function}

Lemma 5.4.1. Let $F:(-1,1) \rightarrow \mathbb{C}$ be a continuous p.d. function. For $\phi$ in $C_{c}^{\infty}(0,1)$ let

$$
(t(\phi))(x)=\phi(1-x), \quad \text { for all } x \in(0,1) .
$$

The operator $F_{\phi} \rightarrow F_{t(\phi)}$ is bounded in $\mathscr{H}_{F}$ iff

$$
\bar{F} \ll F
$$

where $\bar{F}$ is the complex conjugate of $F$, and $\ll$ is the order on p.d. functions, i.e., there is an $A<\infty$ such that

$$
\sum \sum \overline{c_{j}} c_{k} \bar{F}\left(x_{j}-x_{k}\right) \leq A \sum \sum \overline{c_{j}} c_{k} F\left(x_{j}-x_{k}\right),
$$

for all finite systems $\left\{c_{j}\right\}$ and $\left\{x_{j}\right\}$, where $c_{j} \in \mathbb{C}, x_{j} \in(0,1)$.

Proof. It follows from (5.4.2) that $\bar{F} \ll F$ iff there is an $A<\infty$ such that

$$
\left\|\overline{F_{\phi}}\right\|_{\mathscr{H}_{F}} \leq \sqrt{A}\left\|F_{\phi}\right\|_{\mathscr{H}_{F}}
$$

for all $\phi$ in $C_{c}^{\infty}(0,1)$. Since

$$
\begin{aligned}
\left\|F_{t(\phi)}\right\|_{\mathscr{H}_{F}}^{2} & =\int_{0}^{1} \int_{0}^{1} \overline{\phi(1-x)} \phi(1-y) F(x-y) d x d y \\
& =\int_{0}^{1} \int_{0}^{1} \overline{\phi(x)} \phi(y) F(y-x) d x d y \\
& =\left\|\overline{F_{\phi}}\right\|_{\mathscr{H}_{\bar{F}}}^{2}
\end{aligned}
$$

we have established the claim.

Let $M=\left(M_{j k}\right)$ be an $N \times N$ matrix over $\mathbb{C}$. Set

$$
\Re\{M\}=\left(\Re\left\{M_{j k}\right\}\right), \Im\{M\}=\left(\Im\left\{M_{j k}\right\}\right) .
$$

Assume $M^{*}=M$, where $M^{*}$ is the conjugate transpose of $M$, and $M \geq 0$. Recall, $M \geq 0$ iff all eigenvalues of $M$ are $\geq 0$ iff all sub-determinants $\operatorname{det} M_{n} \geq 0, n=$ $1, \ldots, N$, where $M_{n}=\left(M_{j k}\right)_{j, k \leq n}$.

Definition 5.4.2. Let $s(x)=-x$. For a measure $\mu$ on $\mathbb{R}$, let $\mu^{s}=\mu \circ s$.

Lemma 5.4.3. If $F=\widehat{d \mu}$ then $\bar{F}=\widehat{d \mu^{s}}$.

Proof. Suppose $F=\widehat{d \mu}$, then the calculation

$$
\begin{aligned}
\overline{F(x)} & =F(-x)=\int_{\mathbb{R}} e_{\lambda}(-x) d \mu(\lambda) \\
& =\int_{\mathbb{R}} e_{-\lambda}(x) d \mu(\lambda) \\
& =\int_{\mathbb{R}} e_{\lambda}(x) d \mu^{s}(\lambda)=\widehat{d \mu^{s}}(x)
\end{aligned}
$$

establishes the claim.

COROLlary 5.4.4. If $F=\widehat{d \mu}$, then (5.4.3) takes the form

$$
\int_{\mathbb{R}}|\widehat{\phi}(\lambda)|^{2} d \mu^{s}(\lambda) \leq A \int_{\mathbb{R}}|\widehat{\phi}(\lambda)|^{2} d \mu(\lambda)
$$

for all $\phi$ in $C_{c}^{\infty}(0,1)$. 
Proof. A calculation show that

$$
\left\|F_{\phi}\right\|_{\mathscr{H}_{F}}^{2}=\int_{\mathbb{R}}|\widehat{\phi}(\lambda)|^{2} d \mu(\lambda)
$$

and similarly $\left\|\overline{F_{\phi}}\right\|_{\mathscr{H}_{\bar{F}}}^{2}=\int_{\mathbb{R}}|\widehat{\phi}(\lambda)|^{2} d \mu^{s}(\lambda)$, where we used Lemma 5.4.3.

EXAMPLE 5.4.5. If $\mu=\frac{1}{2}\left(\delta_{-1}+\delta_{2}\right)$, then $\mu^{s}=\frac{1}{2}\left(\delta_{-1}+\delta_{2}\right)$. Set

$$
\begin{aligned}
& F(x)=\widehat{\mu}(x)=\frac{1}{2}\left(e^{-i x}+e^{i 2 x}\right), \text { then } \\
& \overline{F(x)}=\widehat{\mu^{s}}(x)=\frac{1}{2}\left(e^{i x}+e^{-i 2 x}\right) .
\end{aligned}
$$

It follows from Corollary 5.4.4 and Lemma 5.4.1 that $\bar{F} \nless F$ and $F \nless \bar{F}$. In fact,

$$
\begin{aligned}
\left\|F_{\phi}\right\|_{\mathscr{H}_{F}}^{2} & =\frac{1}{2}\left(|\widehat{\phi}(-1)|^{2}+|\widehat{\phi}(2)|^{2}\right) \\
\left\|\overline{F_{\phi}}\right\|_{\mathscr{H}_{\bar{F}}}^{2} & =\frac{1}{2}\left(|\widehat{\phi}(1)|^{2}+|\widehat{\phi}(-2)|^{2}\right) .
\end{aligned}
$$

Fix $f \in C_{c}^{\infty}$, such that $f(0)=1, f \geq 0$, and $\int f=1$. Considering $\phi_{n}(x)=$ $\frac{1}{2}\left(e^{-i x}+e^{i 2 x}\right) f\left(\frac{x}{n}\right)$, and $\psi_{n}(x)=\frac{1}{2}\left(e^{-i x}+e^{i 2 x}\right) f\left(\frac{x}{n}\right)$, completes the verification, since $\widehat{\phi_{n}} \rightarrow \mu$ and $\widehat{\psi_{n}} \rightarrow \mu^{s}$.

And similarly, $\bar{F} \nless F$ and $F \nless \bar{F}$, where $F$ is as in Example 5.4.16.

REMARK 5.4.6. In fact, $\bar{F} \ll F$ iff $\mu^{s} \ll \mu$ with Radon-Nikodym derivative $\frac{d \mu^{s}}{d \mu} \in L^{\infty}(\mu)$. See, section 5.3.

Corollary 5.4.7. If $F=\widehat{d \mu}$, then

$$
\begin{aligned}
& \Re\{F\}=\frac{1}{2}\left(\widehat{\mu+\mu^{s}}\right), \text { and } \\
& \Im\{F\}=\frac{1}{2 i}\left(\widehat{\mu-\mu^{s}}\right) .
\end{aligned}
$$

We can rewrite the corollary in the form: If $F=\widehat{d \mu}$, then

$$
\begin{aligned}
& \Re\{F\}(x)=\int_{\mathbb{R}} \cos (\lambda x) d \mu(\lambda), \text { and } \\
& \Im\{F\}(x)=\int_{\mathbb{R}} \sin (\lambda x) d \mu(\lambda) .
\end{aligned}
$$

REMARK 5.4.8. (5.4.5) simply states that if $F$ is positive definite, so is its real part $\Re\{F\}$. But (5.4.6) is deeper: If the function $\lambda$ is in $L^{1}(\mu)$, then

$$
\frac{d}{d x} \Im\{F\}(x)=\int_{\mathbb{R}} \cos (\lambda x) \lambda d \mu(\lambda)
$$

is the cosine transform of $\lambda d \mu(\lambda)$.

Suppose $F$ is p.d. on $(-a, a)$ and $\mu \in \operatorname{Ext}(F)$, i.e., $\mu$ is a finite positive measure satisfying

$$
F(x)=\int_{\mathbb{R}} e_{\lambda}(x) d \mu(x) .
$$

For a finite set $\left\{x_{j}\right\} \in(-a, a)$ let

$$
M:=\left(F\left(x_{j}-x_{k}\right)\right) .
$$


For $c_{j}$ in $\mathbb{C}$ consider

$$
\overline{c^{T}} M c=\sum \sum \overline{c_{j}} c_{k} M_{j k}
$$

The for $\Re\{F\}$, we have

$$
\begin{aligned}
& \sum_{j} \sum_{k} \overline{c_{j}} c_{k} \Re\{F\}\left(x_{j}-x_{k}\right) \\
= & \sum_{j} \sum_{k} \overline{c_{j}} c_{k} \int_{\mathbb{R}}\left(\cos \left(\lambda x_{j}\right) \cos \left(\lambda x_{k}\right)+\sin \left(\lambda x_{j}\right) \sin \left(\lambda x_{k}\right)\right) d \mu(\lambda) \\
= & \int_{\mathbb{R}}\left(|C(\lambda)|^{2}+|S(\lambda)|^{2}\right) d \mu(\lambda) \geq 0,
\end{aligned}
$$

where

$$
\begin{aligned}
& C(\lambda)=C\left(\lambda,\left(x_{j}\right)\right)=\sum_{j} c_{j} \cos \left(\lambda x_{j}\right) \\
& S(\lambda)=S\left(\lambda,\left(x_{j}\right)\right)=\sum_{j} c_{j} \sin \left(\lambda x_{j}\right)
\end{aligned}
$$

for all $\lambda \in \mathbb{R}$. Similarly, for $\Im\{F\}$, we have

$$
\begin{aligned}
& \sum_{j} \sum_{k} \overline{c_{j}} c_{k} \Im\{F\}\left(x_{j}-x_{k}\right) \\
= & \sum_{j} \sum_{k} \overline{c_{j}} c_{k} \int_{\mathbb{R}}\left(\sin \left(\lambda x_{j}\right) \cos \left(\lambda x_{k}\right)-\cos \left(\lambda x_{j}\right) \sin \left(\lambda x_{k}\right)\right) d \mu(\lambda) \\
= & \int_{\mathbb{R}}(\overline{S(\lambda)} C(\lambda)-\overline{C(\lambda)} S(\lambda)) d \mu(\lambda) \\
= & 2 i \int_{\mathbb{R}} \Im\{\overline{S(\lambda)} C(\lambda)\} d \mu(\lambda) .
\end{aligned}
$$

If $\left\{c_{j}\right\} \subset \mathbb{R}$, then $S(\lambda), C(\lambda)$ are real valued and

$$
\sum_{j} \sum_{k} \overline{c_{j}} c_{k} \Im\{F\}\left(x_{j}-x_{k}\right)=0 .
$$

\subsubsection{An application of Bochner's Theorem.}

Lemma 5.4.9. Let $F$ be a continuous positive definite function on some open interval $(-a, a)$. Let $K$ be the real part $\Re\{F\}$ of $F$ and let $L$ be the imaginary part $\Im\{F\}$ of $F$, hence $K$ and $L$ are real valued, $K$ is a continuous positive definite real valued function, in particular $K$ is an even function, and $L$ is an odd function.

Proof. The even/odd claims follow from $F(-x)=\overline{F(x)}$ for $x \in(-a, a)$. For a finite set of points $\left\{x_{j}\right\}_{j=1}^{N}$ in $(-a, a)$ form the matrices

$$
M_{F}=\left(F\left(x_{j}-x_{k}\right)\right)_{j, k=1}^{N}, M_{K}=\left(K\left(x_{j}-x_{k}\right)\right)_{j, k=1}^{N} M_{L}=\left(L\left(x_{j}-x_{k}\right)\right)_{j, k=1}^{N} .
$$

Let $c=\left(c_{j}\right)$ be a vector in $\mathbb{R}^{N}$. Since $L$ is an odd function it follows that $c^{T} M_{L} c=0$, consequently,

$$
c^{T} M_{K} c=c^{T} M_{F} c \geq 0 .
$$

It follows that $K$ is positive definite over the real numbers and therefore also over the complex numbers [Aro50]. 
Definition 5.4.10. We say a signed measure $\mu$ is even, if $\mu(B)=\mu(-B)$ for all $\mu$-measurable sets $B$, where $-B=\{-x: x \in B\}$. Similarly, we say $\mu$ is odd, if $\mu(B)=-\mu(-B)$ for all $\mu-$ measurable sets $B$.

REMARK 5.4.11. Let

$$
\begin{aligned}
\mu_{K}(B) & :=\frac{\mu(B)+\mu(-B)}{2} \text { and } \\
\mu_{L}(B) & :=\frac{\mu(B)-\mu(-B)}{2}
\end{aligned}
$$

for all $\mu$-measurable sets $B$. Then $\mu_{K}$ is an even probability measure and $\mu_{L}$ is an odd real valued measure. If $F=\widehat{d \mu}, K=\widehat{d \mu_{K}}$, and $i L=\widehat{d \mu_{L}}$, then $K$ and $L$ are real valued continuous functions, $F$ and $K$ are continuous positive definite functions, $L$ is a real valued continuous odd function and $F=K+i L$.

Lemma 5.4.12. Suppose $K$ as the Fourier transform of some even probability measure $\mu_{K}$ and $i L$ as the Fourier transform of some odd measure $\mu_{L}$, then $F:=$ $K+i L$ is positive definite iff $\mu:=\mu_{K}+\mu_{L}$ is a probability measure, i.e., iff $\mu(B) \geq 0$ for all Borel set $B$.

ProOF. This is a direct consequence of Bochner's theorem.

Corollary 5.4.13. If $F$ is positive definite, and $\Im\{F\} \neq 0$, then

(i) $F_{m}:=\Re\{F\}+i m \Im\{F\}$, is positive definite for all $-1 \leq m \leq 1$ and

(ii) $F_{m}$ is not positive definite for sufficiently large $m$.

Proof. ( $i$ ) We will use the notation from Remark 5.4.11. If $0<m$ and $\mu_{L}(B)<0$, then

$$
\mu_{m}(B):=\mu_{K}(B)+m \mu_{L}(B) \geq \mu(B) \geq 0 .
$$

The cases where $m<0$ are handled by using that $\bar{F}$ is positive definite.

(ii) Is established using a similar argument.

Corollary 5.4.14. Let $K$ and $L$ be real valued continuous functions on $\mathbb{R}$. Suppose $K$ positive definite and $L$ odd, and let $\mu_{k}$ and $\mu_{L}$ be the correspond even and odd measures. If $K+i m L$ is positive definite for some real $m \neq 0$, then the support of $\mu_{L}$ is a subset of the support of $\mu_{K}$.

Proof. Fix $m \neq 0$. If the support of $\mu_{L}$ is not contained in the support of $\mu_{K}$, then $\mu_{K}(B)+m \mu_{L}(B)<0$ for some $B$.

REMARK 5.4.15. The converse fails, support containment does not imply $\mu_{k}+$ $m \mu_{L}$ is positive for some $m>0$ since $\mu_{K}$ can "decrease" much faster than $\mu_{L}$. Then

EXAMPLE 5.4.16. Let $d \mu(\lambda):=\delta_{-1}+\chi_{\mathbb{R}^{+}}(\lambda) e^{-\lambda} d \lambda$ and set $F:=\left.\widehat{d \mu}\right|_{(-1,1)}$.

$$
\begin{aligned}
& \Re\{F\}(x)=\cos (x)+\frac{1}{1+x^{2}} \\
& \Im\{F\}(x)=-\sin (x)+\frac{x}{1+x^{2}} ;
\end{aligned}
$$

and $D^{(F)}$ in $\mathscr{H}_{F}$ has deficiency indices $(0,0)$. 
Proof. By construction:

$$
\begin{aligned}
F(x) & =\int_{\mathbb{R}} e^{i \lambda x} d \mu(\lambda)=e^{-i x}+\int_{0}^{\infty} e^{i \lambda x}-\lambda d \lambda \\
& =e^{-i x}+\frac{1}{1-i x} ;
\end{aligned}
$$

establishing the first claim.

Consider $u=T_{F} \phi$, for some $\phi \in C_{c}^{\infty}(0,1)$. By (5.4.8)

$$
\begin{aligned}
u(x) & =\int_{0}^{1} \phi(y) F(x-y) d y \\
& =\widehat{\phi}(-1) e^{-i x}+\int_{0}^{1} \phi(y) \frac{1}{1-i(x-y)} d y .
\end{aligned}
$$

Taking two derivatives we get

$$
u^{\prime \prime}(x)=-\widehat{\phi}(-1) e^{-i x}+\int_{0}^{1} \phi(y) \frac{-2}{(1-i(x-y))^{3}} d y .
$$

It follows that $u^{\prime \prime}+u \rightarrow 0$ as $x \rightarrow \pm \infty$, i.e.,

$$
\lim _{|x| \rightarrow \infty}\left|u^{\prime \prime}(x)+u(x)\right|=0 .
$$

A standard approximation argument shows that (5.4.9) holds for all $u \in \mathscr{H}_{F}$.

Equation (5.4.9) rules out that either of $e^{ \pm x}$ is on $\mathscr{H}_{F}$, hence the deficiency indices are $(0,0)$ as claimed.

If $\mu_{K}$ is an even probability measure and $f(x)$ is an odd function, s.t. $-1 \leq$ $f(x) \leq 1$, then $d \mu(x):=(1+f(x)) d \mu_{K}(x)$ is a probability measure. Conversely, we have

Lemma 5.4.17. Let $\mu$ be a probability measure on the Borel sets. There is an even probability measure $\mu_{K}$ and an odd real valued $\mu$-measurable function $f$ with $|f| \leq 1$, such that $d \mu(\lambda)=(1+f(\lambda)) d \mu_{K}(\lambda)$.

Proof. Let $\mu_{K}:=\frac{1}{2}\left(\mu+\mu^{s}\right)$ and $\mu_{L}:=\frac{1}{2}\left(\mu-\mu^{s}\right)$. Clearly, $\mu_{K}$ is an even probability measure and $\mu_{L}$ is an odd real valued measure. Since $\mu_{K}(B)+\mu_{L}(B)=$ $\mu(B) \geq 0$, it follows that $\mu_{K}(B) \geq \mu_{L}(B)$ for all Borel sets $B$.

Applying the Hahn decomposition theorem to $\mu_{L}$ we get sets $P$ and $N$ such that $P \cap N=\emptyset, \mu_{L}(B \cap P) \geq 0$ and $\mu_{L}(B \cap N) \leq 0$ for all $B$. Let

$$
\begin{aligned}
& P^{\prime}:=\{x \in P:-x \in N\} \\
& N^{\prime}:=\{x \in N:-x \in P\} \\
& O^{\prime}:=\left(P \backslash P^{\prime}\right) \cup\left(N \backslash N^{\prime}\right),
\end{aligned}
$$

then $N^{\prime}=-P^{\prime}$ and $\mu_{L}\left(B \cap O^{\prime}\right)=0$ for all $B$. Write

$$
\mu_{L}(B)=\mu_{L}\left(B \cap P^{\prime}\right)+\mu_{L}\left(B \cap N^{\prime}\right) .
$$

Then $\mu_{K}(B) \geq \mu_{K}\left(B \cap P^{\prime}\right) \geq \mu_{L}\left(B \cap P^{\prime}\right)$ and

$$
\begin{aligned}
0 \leq-\mu_{L}\left(B \cap N^{\prime}\right) & =\mu_{L}\left(-\left(B \cap N^{\prime}\right)\right) \\
& =\mu_{L}\left(-B \cap P^{\prime}\right) \\
& \leq \mu_{K}\left(-B \cap P^{\prime}\right) \leq \mu_{K}(B) .
\end{aligned}
$$


Hence, $\mu_{L}$ is absolutely continuous with respect to $\mu_{K}$. Setting $f:=\frac{d \mu_{L}}{d \mu_{K}}$, the Radon-Nikodym derivative of $\mu_{L}$ with respect to $\mu_{K}$, completes the proof.

Corollary 5.4.18. Let $F=\widehat{\mu}$ be a positive definite function with $F(0)=1$. Let $\mu_{K}:=\frac{1}{2}\left(\mu+\mu^{s}\right)$ then $\Re\{F\}(x)=\widehat{\mu_{K}}(x)$ and there is an odd function

$$
-1 \leq f(\lambda) \leq 1
$$

such that $\Im\{F\}(x)=\widehat{f \mu_{K}}(x)$.

Corollary 5.4.19. Let $F$ be a continuous p.d. function on $(-a, a)$. Let $\Re\{F\}$ be the real part of $F$. Then $\mathscr{H}_{F}$ is a subset of $\mathscr{H}_{\Re\{F\}}$. In particular, if $D^{(\Re\{F\})}$ has deficiency indices $(1,1)$ so does $D^{(F)}$.

Proof. Recall, a continuous function $\xi$ is in $\mathscr{H}_{F}$ iff

$$
\left|\int_{0}^{1} \psi(y) \xi(y) d y\right|^{2} \leq A \int_{0}^{1} \int_{0}^{1} \overline{\phi(x)} \phi(y) F(x-y) d x d y .
$$

Since,

$$
\begin{aligned}
\int_{0}^{1} \int_{0}^{1} \overline{\phi(x)} \phi(y) F(x-y) d x d y & =\int_{0}^{1} \int_{0}^{1} \int_{\mathbb{R}} \overline{\phi(x)} \phi(y) e^{-i \lambda(x-y)} d \mu(\lambda) d x d y \\
& =\int_{\mathbb{R}}|\phi(\lambda)|^{2} d \mu(\lambda) \\
& \leq 2 \int_{\mathbb{R}}|\phi(\lambda)|^{2} d \mu_{K}(\lambda) \\
& =2 \int_{0}^{1} \int_{0}^{1} \overline{\phi(x)} \phi(y) K(x-y) d x d y
\end{aligned}
$$

it follows that $\mathscr{H}_{F}$ is contained in $\mathscr{H}_{\Re\{F\}}$. 


\section{CHAPTER 6}

\section{Convolution Products}

A source of interesting measures in probability are constructed as product measures or convolutions; and this includes infinite operations; see for example [IM65, Jor07, KS02, Par09].

Below we study these operations in the contest of our positive definite functions, defined on subsets of groups. For example, most realizations of fractal measures arise as infinite convolutions, see e.g., [DJ10, JP12, JKS12, DJ12, JKS11, JKS08]. Motivated by these applications, we show below that, given a system of continuous positive definite functions $F_{1}, F_{2}, \ldots$, defined on an open subset of a group, we can form well defined products, including infinite products, which are again continuous positive definite. We further show that if finite positive measures $\mu_{i}, i=1,2, \ldots$, are given, $\mu_{i} \in \operatorname{Ext}\left(F_{i}\right)$ then the convolution of the measures $\mu_{i}$ is in $\operatorname{Ext}(F)$ where $F$ is the product of the p.d. functions $F_{i}$. This will be applied later in the Memoir.

Definition 6.0.20. Let $F$ be a continuous positive definite function defined on a subset in $G$ (a locally compact Abelian group). Set

$$
\operatorname{Ext}(F)=\{\mu \in \mathscr{M}(\widehat{G}) \mid \widehat{d \mu} \text { is an extension of } F\} .
$$

In order to study the set $\operatorname{Ext}(F)$ from above, it helps to develop tools. One such tool is convolution, which we outline below. It is also helpful in connection with the study of symmetric spaces, such as the case $G=\mathbb{T}=\mathbb{R} / \mathbb{Z}$ (the circle group), versus extensions to the group $\mathbb{R}$.

Let $G$ be a locally compact group, and let $\Omega$ be a non-empty, connected and open subset in $G$. Now consider systems of p.d. and continuous functions on the set $\Omega^{-1} \Omega$. Specifically, let $F_{i}$ be two or more p.d. continuous functions on $\Omega^{-1} \Omega$; possibly an infinite family, so $F_{1}, F_{2}, \ldots$, all defined on $\Omega^{-1} \Omega$. As usual, we normalize our p.d. functions $F_{i}(e)=1$, where $e$ is the unit element in $G$.

Lemma 6.0.21. Form the point-wise product $F$ of any system of p.d. functions $F_{i}$ on $\Omega^{-1} \Omega$; then $F$ is again p.d. and continuous on the set $\Omega^{-1} \Omega$.

Proof. This is an application of a standard lemma about p.d. kernels, see e.g., [BCR84]. From this, we conclude that $F$ is again a continuous and positive definite function on $\Omega^{-1} \Omega$.

If we further assume that $G$ is also Abelian, and so $G$ is locally compact abelian, then the spectral theory takes a more explicit form.

Lemma 6.0.22. Assume Ext $\left(F_{i}\right)$ for $i=1,2, \ldots$ are non-empty. For any system of individual measures $\mu_{i} \in \operatorname{Ext}\left(F_{i}\right)$ we get that the resulting convolutionproduct measure $\mu$ formed from the factors $\mu_{i}$ by the convolution in $G$, is in $\operatorname{Ext}(F)$ 
Proof. This is an application of our results in sections 2.4-2.5.

REMARK 6.0.23. In some applications the convolution $\mu_{1} * \mu_{2}$ makes sense even if only one of the measures is finite.

Application. The case $G=\mathbb{R}$. Let $\mu_{1}$ be the Dirac-comb ([Ric03, Cór89])

$$
d \mu_{1}:=\sum_{n \in \mathbb{Z}} \delta(\lambda-n), \lambda \in \mathbb{R}
$$

let $\Phi \geq 0, \Phi \in L^{1}(\mathbb{R})$, and assume $\int_{\mathbb{R}} \Phi(\lambda) d \lambda=1$. Set $d \mu_{2}=\Phi(\lambda) d \lambda$, where $d \lambda=$ Lebesgue measure on $\mathbb{R}$; then $\mu_{1} * \mu_{2}$ yields the following probability measure on $\mathbb{T}=\mathbb{R} / \mathbb{Z}$ : Set

$$
\Phi_{\text {per }}(\lambda)=\sum_{n \in \mathbb{Z}} \Phi(\lambda-n) ;
$$

then $\Phi_{\text {per }}(\lambda) \in L^{1}(\mathbb{T}, d t)$, where $d t=$ Lebesgue measure on $\mathbb{T}$, i.e., if $f(\lambda+n)=f(n), \forall n \in \mathbb{Z}, \forall \lambda \in \mathbb{R}$, then $f$ defines a function on $\mathbb{T}$, and $\int_{\mathbb{T}} f d t=\int_{0}^{1} f(t) d t$. We get

$$
d\left(\mu_{1} * \mu_{2}\right)=\Phi_{\text {per }}(\cdot) d t \text { on } \mathbb{T} .
$$

Proof. We have

$$
\begin{aligned}
1 & =\int_{-\infty}^{\infty} \Phi(\lambda) d \lambda=\sum_{n \in \mathbb{Z}} \int_{n}^{n+1} \Phi(\lambda) d \lambda \\
& =\int_{0}^{1} \sum_{n \in \mathbb{Z}} \Phi(\lambda-n) d \lambda \\
& =\int_{\mathbb{T}} \Phi_{p e r}(t) d t
\end{aligned}
$$

We now proceed to study the relations between the other items in our analysis, the RKHSs $\mathscr{H}_{F_{i}}$, for $i=1,2, \ldots$; and computing $\mathscr{H}_{F}$ from the RKHSs $\mathscr{H}_{F_{i}}$.

We further study the associated unitary representations of $G$ when $\operatorname{Ext}\left(F_{i}\right)$, $i=1,2, \ldots$ are non-empty?

As an application, we get infinite convolutions, and they are fascinating; include many fractal measures of course.

In the case of $\mathbb{G}=\mathbb{R}$ we will study the connection between deficiency index values in $\mathscr{H}_{F}$ as compared to those of the factor RKHSs $F_{i}$. 


\section{CHAPTER 7}

\section{Models for Operator Extensions}

A special case of our extension question for continuous positive definite (p.d.) functions on a fixed finite interval $|x|<a$ in $\mathbb{R}$ is the following: It offers a spectral model representation for ALL Hermitian operators with dense domain in Hilbert space and with deficiency indices $(1,1)$.

Specifically, on $\mathbb{R}$, all the partially defined continuous p.d. functions extend, and we can make a translation of our p.d. problem into the problem of finding all $(1,1)$ restrictions selfadjoint operators.

By the Spectral theorem, every selfadjoint operator with simple spectrum has a representation as a multiplication operator $M_{\lambda}$ in some $L^{2}(\mathbb{R}, \mu)$ for some probability measure $\mu$ on $\mathbb{R}$. So this accounts for all Hermitian restrictions operators with deficiency indices $(1,1)$.

So the problem we have been studying for just the case of $G=\mathbb{R}$ is the case of finding spectral representations for ALL Hermitian operators with dense domain in Hilbert space having deficiency indices $(1,1)$.

\subsection{Model for Restrictions of Continuous p.d. Functions on $\mathbb{R}$}

Let $\mathscr{H}$ be a Hilbert space, $A$ a skew-adjoint operator, $A^{*}=-A$, which is

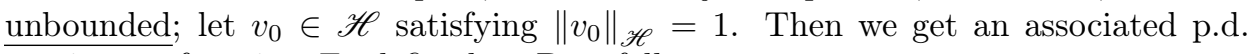
continuous function $F_{A}$ defined on $\mathbb{R}$ as follows:

$$
F_{A}(t):=\left\langle v_{0}, e^{t A} v_{0}\right\rangle=\left\langle v_{0}, U_{A}(t) v_{0}\right\rangle, t \in \mathbb{R},
$$

where $U_{A}(t)=e^{t A}$ is a unitary representation of $\mathbb{R}$. Note that we define $U(t)=$ $U_{A}(t)=e^{t A}$ by the spectral theorem. Note (7.1.1) holds for all $t \in \mathbb{R}$.

Let $P_{U}(\cdot)$ be the projection-valued measure (PVM) of $A$, then

$$
U(t)=\int_{-\infty}^{\infty} e^{i \lambda t} P_{U}(d \lambda), \forall t \in \mathbb{R}
$$

Lemma 7.1.1. Setting

$$
d \mu=\left\|P_{U}(d \lambda) v_{0}\right\|^{2}
$$

we then get

$$
F_{A}(t)=\widehat{d \mu}(t), \forall t \in \mathbb{R}
$$

Moreover, every probability measure $\mu$ on $\mathbb{R}$ arises this way.

Proof. By (7.1.1),

$$
\begin{aligned}
F_{A}(t) & =\int e^{i t \lambda}\left\langle v_{0}, P_{U}(d \lambda) v_{0}\right\rangle \\
& =\int e^{i t \lambda}\left\|P_{U}(d \lambda) v_{0}\right\|^{2}
\end{aligned}
$$




$$
=\int e^{i t \lambda} d \mu(\lambda)
$$

Lemma 7.1.2. For Borel functions $f$ on $\mathbb{R}$, let

$$
f(A)=\int_{\mathbb{R}} f(\lambda) P_{U}(d \lambda)
$$

be given by functional calculus. We note that

$$
v_{0} \in \operatorname{dom}(f(A)) \Longleftrightarrow f \in L^{2}(\mu)
$$

where $\mu$ is the measure in (7.1.3). Then

$$
\left\|f(A) v_{0}\right\|^{2}=\int_{\mathbb{R}}|f|^{2} d \mu .
$$

Proof. The lemma follows from

$$
\begin{array}{rlrl}
\left\|f(A) v_{0}\right\|^{2} & =\left\|\int_{\mathbb{R}} f(\lambda) P_{U}(d \lambda) v_{0}\right\|^{2} & & (\text { by }(7.1 .5)) \\
& =\int|f(\lambda)|^{2}\left\|P_{U}(d \lambda) v_{0}\right\|^{2} & \\
& =\int|f(\lambda)|^{2} d \mu(\lambda) . & \quad(\text { by }(7.1 .3))
\end{array}
$$

Now we consider restriction of $F_{A}$ to, say $(-1,1)$, i.e.,

$$
F(\cdot)=\left.F_{A}\right|_{(-1,1)}(\cdot)
$$

Lemma 7.1.3. Let $\mathscr{H}_{F}$ be the RKHS computed for $F$ in (7.1.4); and for $\varphi \in$ $C_{c}(0,1)$, set $F_{\varphi}=$ the generating vectors in $\mathscr{H}_{F}$, as usual. Set

$$
U(\varphi):=\int_{0}^{1} \varphi(y) U(-y) d y
$$

where $d y=$ Lebesgue measure on $(0,1)$; then

$$
F_{\varphi}(x)=\left\langle v_{0}, U(x) U(\varphi) v_{0}\right\rangle, \forall x \in(0,1) .
$$

Proof. We have

$$
\begin{aligned}
F_{\varphi}(x) & =\int_{0}^{1} \varphi(y) F(x-y) d y \\
& =\int_{0}^{1} \varphi(y)\left\langle v_{0}, U_{A}(x-y) v_{0}\right\rangle d y \quad(\text { by }(7.1 .1)) \\
& =\left\langle v_{0}, U_{A}(x) \int_{0}^{1} \varphi(y) U_{A}(-y) v_{0} d y\right\rangle \\
& =\left\langle v_{0}, U_{A}(x) U(\varphi) v_{0}\right\rangle \quad(\text { by }(7.1 .9)) \\
& =\left\langle v_{0}, U(\varphi) U_{A}(x) v_{0}\right\rangle
\end{aligned}
$$

for all $x \in(0,1)$, and all $\varphi \in C_{c}(0,1)$. 
Corollary 7.1.4. Let $A, U(t)=e^{t A}, v_{0} \in \mathscr{H}, \varphi \in C_{c}(0,1)$, and $F$ p.d. on $(0,1)$ be as above; let $\mathscr{H}_{F}$ be the RKHS of F; then, for the inner product in $\mathscr{H}_{F}$, we have

$$
\left\langle F_{\varphi}, F_{\psi}\right\rangle_{\mathscr{H}_{F}}=\left\langle U(\varphi) v_{0}, U(\psi) v_{0}\right\rangle_{\mathscr{H}}, \forall \varphi, \psi \in C_{c}(0,1) .
$$

Proof. Note that

$$
\begin{aligned}
\left\langle F_{\varphi}, F_{\psi}\right\rangle_{\mathscr{H}_{F}} & =\int_{0}^{1} \int_{0}^{1} \overline{\varphi(x)} \psi(y) F(x-y) d x d y \\
& \left.=\int_{0}^{1} \int_{0}^{1} \overline{\varphi(x)} \psi(y)\left\langle v_{0}, U_{A}(x-y) v_{0}\right\rangle_{\mathscr{H}} d x d y \quad \text { (by }(7.1 .8)\right) \\
& =\int_{0}^{1} \int_{0}^{1}\left\langle\varphi(x) U_{A}(-x) v_{0}, \psi(y) U_{A}(-y) v_{0}\right\rangle_{\mathscr{H}} d x d y \\
& =\left\langle U(\varphi) v_{0}, U(\psi) v_{0}\right\rangle_{\mathscr{H}} \quad(\text { by }(7.1 .9))
\end{aligned}
$$

Corollary 7.1.5. Set $\varphi^{\#}(x)=\overline{\varphi(-x)}, x \in \mathbb{R}, \varphi \in C_{c}(\mathbb{R})$, or in this case, $\varphi \in C_{c}(0,1)$; then we have:

$$
\left\langle F_{\varphi}, F_{\psi}\right\rangle_{\mathscr{H}_{F}}=\left\langle v_{0}, U\left(\varphi^{\#} * \psi\right) v_{0}\right\rangle_{\mathscr{H}}, \forall \varphi, \psi \in C_{c}(0,1) .
$$

ProOF. Immediate from (7.1.11) and Fubini.

COROLlary 7.1.6. Let $F$ and $\varphi \in C_{c}(0,1)$ be as above; then in the $R K H S \mathscr{H}_{F}$ we have:

$$
\left\|F_{\varphi}\right\|_{\mathscr{H}_{F}}^{2}=\left\|U(\varphi) v_{0}\right\|_{\mathscr{H}}^{2}=\int|\widehat{\varphi}|^{2} d \mu
$$

where $\mu$ is the measure in (7.1.3). $\widehat{\varphi}=$ Fourier transform: $\widehat{\varphi}(\lambda)=\int_{0}^{1} e^{-i \lambda x} \varphi(x) d x$, $\lambda \in \mathbb{R}$.

Proof. Immediate from (7.1.12); indeed:

$$
\begin{aligned}
\left\|F_{\varphi}\right\|_{\mathscr{H}_{F}}^{2} & =\int_{0}^{1} \int_{0}^{1} \overline{\varphi(x)} \varphi(y) \int_{\mathbb{R}} e_{\lambda}(x-y) d \mu(\lambda) \\
& =\int_{\mathbb{R}}|\widehat{\varphi}(\lambda)|^{2} d \mu(\lambda), \forall \varphi \in C_{c}(0,1) .
\end{aligned}
$$

Corollary 7.1.7. Every Borel probability measure $\mu$ on $\mathbb{R}$ arises this way.

Proof. We shall need to following:

Lemma 7.1.8. Let $A, \mathscr{H},\left\{U_{A}(t)\right\}_{t \in \mathbb{R}}, v_{0} \in \mathscr{H}$ be as above; and set

$$
d \mu=d \mu_{A}(\cdot)=\left\|P_{U}(\cdot) v_{0}\right\|^{2}
$$

as in (7.1.3). Assume $v_{0}$ is cyclic; then $W_{\mu} f(A) v_{0}=f$ defines a unitary isomorphism $W_{\mu}: \mathscr{H} \rightarrow L^{2}(\mu)$; and

$$
W_{\mu} U_{A}(t)=e^{i t \cdot} W_{\mu}
$$

where $e^{i t .}$ is seen as a multiplication operator in $L^{2}(\mu)$. More precisely:

$$
\left(W_{\mu} U(t) \xi\right)(\lambda)=e^{i t \lambda}\left(W_{\mu} \xi\right)(\lambda), \forall t, \lambda \in \mathbb{R}, \forall \xi \in \mathscr{H} .
$$

(We say that the isometry $W_{\mu}$ intertwines the two unitary one-parameter groups.) 
Proof. Since $v_{0}$ is cyclic, it is enough to consider $\xi \in \mathscr{H}$ of the following form: $\xi=f(A) v_{0}$, with $f \in L^{2}(\mu)$, see (7.1.6) in Lemma 7.1.2. Then

$$
\begin{gathered}
\|\xi\|_{\mathscr{H}}^{2}=\int_{\mathbb{R}}|f(\lambda)|^{2} d \mu(\lambda), \text { so } \\
\left\|W_{\mu} \xi\right\|_{L^{2}(\mu)}=\|\xi\|_{\mathscr{H}}(\Longleftrightarrow(7.1 .17))
\end{gathered}
$$

For the adjoint operator $W_{\mu}^{*}: L^{2}(\mathbb{R}, \mu) \rightarrow \mathscr{H}$, we have

$$
W_{\mu}^{*} f=f(A) v_{0}
$$

see (7.1.5)-(7.1.7). Note that $f(A) v_{0} \in \mathscr{H}$ is well-defined for all $f \in L^{2}(\mu)$. Also $W_{\mu}^{*} W_{\mu}=I_{\mathscr{H}}, W_{\mu} W_{\mu}^{*}=I_{L^{2}(\mu)}$.

Proof of (7.1.16). Take $\xi=f(A) v_{0}, f \in L^{2}(\mu)$, and apply the previous lemma, we have

$$
W_{\mu} U(t) \xi=W_{\mu} U(t) f(A)_{0}=W_{\mu}\left(e^{i t \cdot} f(\cdot)\right)(A) v_{0}=e^{i t \cdot f(\cdot)=e^{i t \cdot} W_{\mu} \xi ;}
$$

or written differently:

$$
W_{\mu} U(t)=M_{e^{i t}} \cdot W_{\mu}, \forall t \in \mathbb{R}
$$

where $M_{e^{i t}}$. is the multiplication operator by $e^{i t}$.

REMARK 7.1.9. Deficiency indices $(1,1)$ occur for probability measures $\mu$ on $\mathbb{R}$ such that

$$
\int_{\mathbb{R}}|\lambda|^{2} d \mu(\lambda)=\infty
$$

See examples below.

\begin{tabular}{|c|c|c|}
\hline measure & condition $(7.1 .18)$ & deficiency indices \\
\hline$\mu_{1}$ & $\int_{\mathbb{R}}|\lambda|^{2} e^{-|\lambda|} d \lambda<\infty$ & $(0,0)$ \\
\hline$\mu_{2}$ & $\int_{\mathbb{R}}|\lambda|^{2}\left(\frac{\sin \pi \lambda}{\pi \lambda}\right)^{2} d \lambda=\infty$ & $(1,1)$ \\
\hline$\mu_{3}$ & $\int_{\mathbb{R}}|\lambda|^{2} \frac{d \lambda}{\pi\left(1+\lambda^{2}\right)}=\infty$ & $(1,1)$ \\
\hline$\mu_{4}$ & $\int_{\mathbb{R}}|\lambda|^{2} \chi_{(-1,1)}(\lambda)(1-|\lambda|) d \lambda<\infty$ & $(0,0)$ \\
\hline$\mu_{5}$ & $\int_{\mathbb{R}}|\lambda|^{2} \frac{1}{\sqrt{2 \pi}} e^{-\lambda^{2} / 2} d \lambda=1<\infty$ & $(0,0)$ \\
\hline
\end{tabular}

TABLE 7.1.1. Application of Theorem 7.1.10 to Table 3.1.1. 

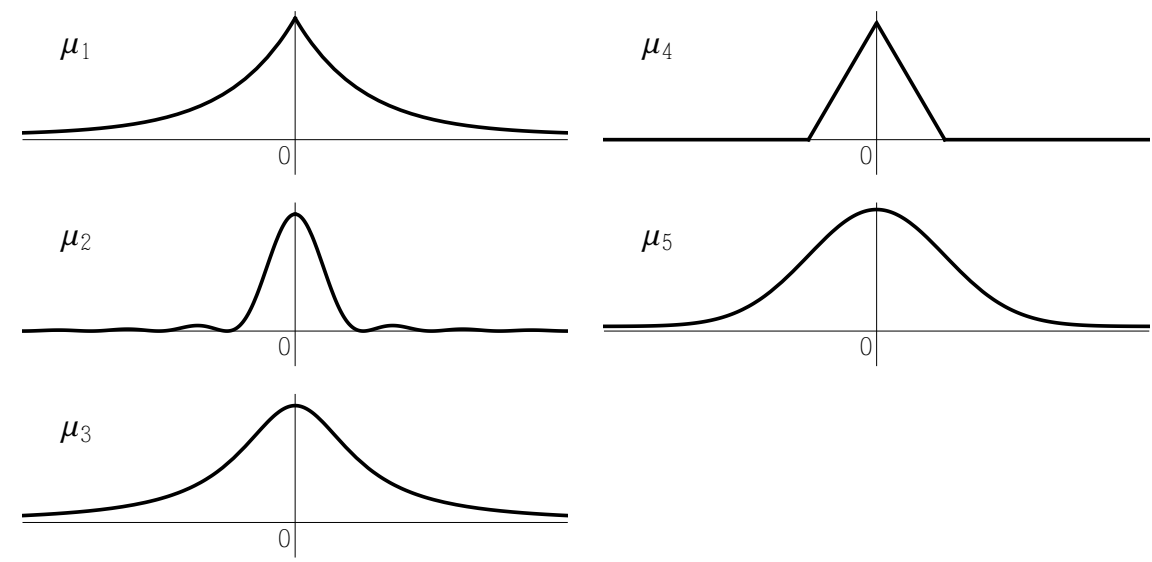

Figure 7.1.1. The measures $\mu_{i} \in \operatorname{Ext}\left(F_{i}\right)$ extending p.d. functions $F_{i}$ in Table $3.1 .1, i=1,2, \ldots 5$.

Summary: restrictions with deficiency indices $(1,1)$.

THEOREM 7.1.10. If $\mu$ is a fixed probability measure on $\mathbb{R}$, then the following two conditions are equivalent:

(1) $\int_{\mathbb{R}} \lambda^{2} d \mu(\lambda)=\infty$;

(2) The set

$$
\operatorname{dom}(S)=\left\{f \in L^{2}(\mu) \mid \lambda f \in L^{2}(\mu) \text { and } \int_{\mathbb{R}}(\lambda+i) f(\lambda) d \mu(\lambda)=0\right\}
$$

is the dense domain of a restriction operator $S \subset M_{\lambda}$ with deficiency indices $\overline{(1,1)}$, and the deficiency space $D E F_{+}=\mathbb{C} 1,(1=$ the constant function 1 in $L^{2}(\mu)$.)

\subsection{A Model of ALL Deficiency Index- $(1,1)$ Operators}

LEMMA 7.2.1. Let $\mu$ be a Borel probability measure on $\mathbb{R}$, and denote $L^{2}(\mathbb{R}, d \mu)$ by $L^{2}(\mu)$. then we have TFAE:

(1)

$$
\int_{\mathbb{R}}|\lambda|^{2} d \mu(\lambda)=\infty
$$

(2) the following two subspaces in $L^{2}(\mu)$ are dense (in the $L^{2}(\mu)$-norm):

$$
\left\{f \in L^{2}(\mu) \mid[(\lambda \pm i) f(\lambda)] \in L^{2}(\mu) \text { and } \int(\lambda \pm i) f(\lambda) d \mu(\lambda)=0\right\}
$$

where $i=\sqrt{-1}$.

Proof. See [Jør81].

REMARK 7.2.2. If (7.2.1) holds, then the two dense subspaces $\mathscr{D}_{ \pm} \subset L^{2}(\mu)$ in (7.2.2) form the dense domain of a restriction $S$ of $M_{\lambda}$ in $L^{2}(\mu)$; and this restriction has deficiency indices $(1,1)$. Moreover, all Hermitian operators having deficiency indices $(1,1)$ arise this way. 
Assume (7.2.1) holds; then the subspace

$$
\mathscr{D}=\left\{f \in L^{2}(\mu) \mid(\lambda+i) f \in L^{2}(\mu) \text { and } \int(\lambda+i) f(\lambda) d \mu(\lambda)=0\right\}
$$

is a dense domain of a restricted operator of $M_{\lambda}$, so $S \subset M_{\lambda}$, and $S$ is Hermitian.

LEMMA 7.2.3. With $i=\sqrt{-1}$, set

$\operatorname{dom}(S)=\left\{f \in L^{2}(\mu) \mid \lambda f \in L^{2}(\mu)\right.$ and $\left.\int(\lambda+i) f(\lambda) d \mu(\lambda)=0\right\}$

then $S \subset M_{\lambda} \subset S^{*}$; and the deficiency subspaces $D E F_{ \pm}$are as follow:

$$
\begin{aligned}
& D E F_{+}=\text {the constant function in } L^{2}(\mu)=\mathbb{C} 1 \\
& D E F_{-}=\operatorname{span}\left\{\frac{\lambda-i}{\lambda+i}\right\}_{\lambda \in \mathbb{R}} \subseteq L^{2}(\mu)
\end{aligned}
$$

where DEF_ is also a 1-dimensional subspace in $L^{2}(\mu)$.

Proof. Let $f \in \operatorname{dom}(S)$, then, by definition,

$$
\begin{gathered}
\int_{\mathbb{R}}(\lambda+i) f(\lambda) d \mu(\lambda)=0 \text { and so } \\
\langle 1,(S+i I) f\rangle_{L^{2}(\mu)}=\int_{\mathbb{R}}(\lambda+i) f(\lambda) d \mu(\lambda)=0
\end{gathered}
$$

hence (7.2.4) follows.

Note we have formula (7.2.3) for $\operatorname{dom}(S)$. Moreover $\operatorname{dom}(S)$ is dense in $L^{2}(\mu)$ because of (7.2.2) in Lemma 7.2.1.

Now to (7.2.5): Let $f \in \operatorname{dom}(S)$; then

$$
\begin{aligned}
\left\langle\frac{\lambda-i}{\lambda+i},(S-i I) f\right\rangle_{L^{2}(\mu)} & =\int_{\mathbb{R}}\left(\frac{\lambda+i}{\lambda-i}\right)(\lambda-i) f(\lambda) d \mu(\lambda) \\
& =\int_{\mathbb{R}}(\lambda+i) f(\lambda) d \mu(\lambda)=0
\end{aligned}
$$

again using the definition of $\operatorname{dom}(S)$ in (7.2.3).

We have established a representation for all Hermitian operators with dense domain in a Hilbert space, and having deficiency indices $(1,1)$. In particular, we have justified the answers in Table 3.1.1 for $F_{i}, i=1, \ldots, 5$.

To further emphasize to the result we need about deficiency indices $(1,1)$, we have the following:

THEOREM 7.2.4. Let $\mathscr{H}$ be a separable Hilbert space, and let $S$ be a Hermitian operator with dense domain in $\mathscr{H}$. Suppose the deficiency indices of $S$ are $(d, d)$; and suppose one of the selfadjoint extensions of $S$ has simple spectrum.

Then the following two conditions are equivalent:

(1) $d=1$;

(2) for each of the selfadjoint extensions $T$ of $S$, we have a unitary equivalence between $(S, \mathscr{H})$ on the one hand, and a system $\left(S_{\mu}, L^{2}(\mathbb{R}, \mu)\right)$ on the other, where $\mu$ is a Borel probability measure on $\mathbb{R}$. Moreover,

$$
\operatorname{dom}\left(S_{\mu}\right)=\left\{f \in L^{2}(\mu) \mid \lambda f(\cdot) \in L^{2}(\mu), \text { and } \int_{\mathbb{R}}(\lambda+i) f(\lambda) d \mu(\lambda)=0\right\} \text {, }
$$


and

$$
\left(S_{\mu} f\right)(\lambda)=\lambda f(\lambda), \forall f \in \operatorname{dom}\left(S_{\mu}\right), \forall \lambda \in \mathbb{R} .
$$

In case $\mu$ satisfies condition (7.2.8), then the constant function 1 (in $L^{2}(\mathbb{R}, \mu)$ ) is in the domain of $S_{\mu}^{*}$, and

$$
S_{\mu}^{*} 1=i 1
$$

i.e., $\left(S_{\mu}^{*} \mathbf{1}\right)(\lambda)=i$, a.a. $\lambda$ w.r.t. $d \mu$.

Proof. For the implication $(2) \Rightarrow(1)$, see Lemma 7.2.3.

$(1) \Rightarrow(2)$. Assume that the operator $S$, acting in $\mathscr{H}$ is Hermitian with deficiency indices $(1,1)$. This means that each of the two subspaces $D E F_{ \pm} \subset \mathscr{H}$ is onedimensional, where

$$
D E F_{ \pm}=\left\{h_{ \pm} \in \operatorname{dom}\left(S^{*}\right) \mid S^{*} h_{ \pm}= \pm i h_{ \pm}\right\} .
$$

Now pick a selfadjoint extension, say $T$, extending $S$. We have

$$
S \subseteq T=T^{*} \subseteq S^{*}
$$

where " $\subseteq$ " in (7.2.11) means "containment of the respective graphs."

Now set $U(t)=e^{i t T}, t \in \mathbb{R}$, and let $P_{U}(\cdot)$ be the corresponding projectionvalued measure, i.e., we have:

$$
U(t)=\int_{\mathbb{R}} e^{i t \lambda} P_{U}(d \lambda), \forall t \in \mathbb{R} .
$$

Using the assumption (1), and (7.2.10), it follows that there is a vector $h_{+} \in \mathscr{H}$ such that $\left\|h_{+}\right\|_{\mathscr{H}}=1, h_{+} \in \operatorname{dom}\left(S^{*}\right)$, and $S^{*} h_{+}=i h_{+}$. Now set

$$
d \mu(\lambda):=\left\|P_{U}(d \lambda) h_{+}\right\|_{\mathscr{H}}^{2} .
$$

Using (7.2.12), we then verify that there is a unitary (and isometric) isomorphism of $L^{2}(\mu) \stackrel{W}{\longrightarrow} \mathscr{H}$ given by

$$
W f=f(T) h_{+}, \forall f \in L^{2}(\mu) ;
$$

where $f(T)=\int_{\mathbb{R}} f(T) P_{U}(d \lambda)$ is the functional calculus applied to the selfadjoint operator $T$. Hence

$$
\begin{aligned}
\|W f\|_{\mathscr{H}}^{2} & =\left\|f(T) h_{+}\right\|_{\mathscr{H}}^{2} \\
& =\int_{\mathbb{R}}|f(\lambda)|^{2}\left\|P_{U}(d \lambda) h_{+}\right\|^{2} \\
& =\int_{\mathbb{R}}|f(\lambda)|^{2} d \mu(\lambda) \quad \text { (by 7.2.13) } \\
& =\|f\|_{L^{2}(\mu)}^{2} .
\end{aligned}
$$

To see that $W$ in (7.2.14) is an isometric isomorphism of $L^{2}(\mu)$ onto $\mathscr{H}$, we use the assumption that $T$ has simple spectrum.

Now set

$$
\begin{aligned}
& S_{\mu}:=W^{*} S W \\
& T_{\mu}:=W^{*} T W .
\end{aligned}
$$

We note that $T_{\mu}$ is then the multiplication operator $M$ in $L^{2}(\mathbb{R}, \mu)$, given by

$$
(M f)(\lambda)=\lambda f(\lambda), \forall f \in L^{2}(\mu)
$$


such that $\lambda f \in L^{2}(\mu)$. This assertion is immediate from (7.2.14) and (7.2.13).

To finish the proof, we compute the integral in (7.2.7) in the theorem, and we use the intertwining properties of the isomorphism $W$ from (7.2.14). Indeed, we have

$$
\begin{array}{rll}
\int_{\mathbb{R}}(\lambda+i) f(\lambda) d \mu(\lambda)= & \langle\mathbf{1},(M+i I) f\rangle_{L^{2}(\mu)} \\
= & \langle W \mathbf{1}, W(M+i I) f\rangle_{\mathscr{H}} \\
\stackrel{(7.2 .13)}{=} & \left\langle h_{+},(T+i I) W f\right\rangle_{\mathscr{H}} .
\end{array}
$$

Hence $W f \in \operatorname{dom}(S) \Longleftrightarrow f \in \operatorname{dom}\left(S_{\mu}\right)$, by (7.2.15); and, so for $W f \in \operatorname{dom}(S)$, the RHS in (7.2.18) yields $\left\langle\left(S^{*}-i I\right) h_{+}, W f\right\rangle_{\mathscr{H}}=0$; and the assertion (2) in the theorem follows.

7.2.1. Momentum Operators in $L^{2}(0,1)$. What about our work on momentum operators in $L^{2}(0,1)$ ? They have deficiency indices $(1,1)$; and there is the family of measures $\mu$ on $\mathbb{R}$ :

Fix $0 \leq \theta<1$, fix

$$
w_{n}>0, \sum_{n \in \mathbb{Z}} w_{n}=1
$$

and let $\mu=\mu_{(\theta, w)}$ as follows:

$$
d \mu=\sum_{n \in \mathbb{Z}} w_{n} \delta_{\theta+n}
$$

such that

$$
\sum_{n \in \mathbb{Z}} n^{2} w_{n}=\infty
$$

In this case, there is the bijective $L^{2}(\mathbb{R}, d \mu) \longleftrightarrow\left\{\xi_{n}\right\}_{n \in \mathbb{Z}}, \xi \in \mathbb{C}$ s.t.

$$
\sum_{n \in \mathbb{Z}}\left|\xi_{n}\right|^{2} w_{n}<\infty
$$

We further assume that

$$
\sum n^{2} w_{n}=\infty
$$

The trick is to pick a representation in which $v_{0}$ in Lemma 7.1.2, i.e., (7.1.5)-(7.1.7): $\mathscr{H}$, cyclic vector $v_{0} \in \mathscr{H},\left\|v_{0}\right\|_{\mathscr{H}}=1,\left\{U_{A}(t)\right\}_{t \in \mathbb{R}}$.

Lemma 7.2.5. The $(1,1)$ index condition is the case of the momentum operator in $L^{2}(0,1)$. To see this we set $v_{0}=e^{x}$, or $v_{0}=e^{2 \pi x}$.

Proof. Defect vector in $\mathscr{H}=L^{2}(0,1)$, say $e^{2 \pi x}$ :

$$
v_{0}(x)=\sum_{n \in \mathbb{Z}} c_{n} e_{n}^{\theta}(x)
$$

with

$$
w_{n} \sim \frac{\left(\cos (2 \pi(\theta+n))-e^{-2 \pi}\right)^{2}+\sin ^{2}(2 \pi(\theta+n))}{1+(\theta+n)^{2}}
$$

and so the $(1,1)$ condition holds, i.e.,

$$
\sum_{n \in \mathbb{Z}} n^{2} w_{n}=\infty
$$


We specialize to $\mathscr{H}=L^{2}(0,1)$, and the usual momentum operator in $(0,1)$ with the selfadjoint extensions. Fix $\theta, 0 \leq \theta<1$, we have an ONB in $\mathscr{H}=L^{2}(0,1)$, $e_{k}^{\theta}(x):=e^{i 2 \pi(\theta+k) x}$, and

$$
v_{0}(x)=\sum_{k \in \mathbb{Z}} c_{k} e_{k}^{\theta}(x), \sum_{k \in \mathbb{Z}}\left|c_{k}\right|^{2}=1 .
$$

Fact. $v_{0}$ is cyclic for $\left\{U_{A_{\theta}}(t)\right\}_{t \in \mathbb{R}} \Longleftrightarrow c_{k} \neq 0, \forall k \in \mathbb{Z}$; then set $w_{k}=\left|c_{k}\right|^{2}$, and the conditions (7.2.19)-(7.2.21) holds. Reason: the measure $\mu=\mu_{v_{0}, \theta}$ depends on the choice of $v_{0}$. The non-trivial examples here $v_{0} \longleftrightarrow\left\{c_{k}\right\}_{k \in \mathbb{Z}}, c_{k} \neq 0, \forall k$, is cyclic, i.e.,

$$
c l \operatorname{span}\left\{U_{A_{\theta}}(t) v_{0} \mid t \in \mathbb{R}\right\}\left(=\mathscr{H}=L^{2}(0,1)\right)
$$

Lemma 7.2.6. The measure $\mu$ in (7.2.20) is determined as:

$$
\begin{aligned}
F_{\theta}(t) & =\left\langle v_{0}, U_{A_{\theta}}(t) v_{0}\right\rangle \\
& =\sum_{k \in \mathbb{Z}} e^{i t 2 \pi(\theta+k)} w_{k} \\
& =\int_{\mathbb{R}} e^{i t \lambda} d \mu(\lambda)
\end{aligned}
$$

where $\mu$ is as in (7.2.20); so purely atomic.

Note that our boundary conditions for the selfadjoint extensions of the minimal momentum operator are implied by (7.2.22), i.e.,

$$
f_{0}(x+1)=e^{i 2 \pi \theta} f_{0}(x), \forall x \in \mathbb{R} .
$$

It is implied by choices of $\theta$ s.t.

$$
f_{0}=\sum_{n \in \mathbb{Z}} B_{n} e_{n}^{\theta}(x), \sum_{n \in \mathbb{Z}}\left|B_{n}\right|^{2}<\infty .
$$

7.2.2. Restriction Operators. In this representation, the restriction operators are represented by sequence $\left\{f_{k}\right\}_{k \in \mathbb{Z}}$ s.t. $\sum\left|f_{k}\right|^{2} w_{k}<\infty$, so use restriction the selfadjoint operator corresponds to the $\theta$-boundary conditions $(7.2 .23), \operatorname{dom}(S)$ where $S$ is the Hermitian restriction operator, i.e., $S \subset s . a . \subset S^{*}$. It has its dense domain $\operatorname{dom}(S)$ as follows: $\left(f_{k}\right) \in \operatorname{dom}(S) \Longleftrightarrow\left(k f_{k}\right) \in l^{2}(\mathbb{Z}, w)$, and

$$
\sum_{k \in \mathbb{Z}}(\theta+k+i) f_{k} w_{k}=0 ; i=\sqrt{-1} .
$$

Comparison with (7.2.3) in the general case. What is special about this case $\mathscr{H}=L^{2}(0,1)$ and the usual boundary conditions at the endpoints is that the family of measures $\mu$ on $\mathbb{R}$ are purely atomic; see (7.2.20)

$$
d \mu=\sum_{k \in \mathbb{Z}} w_{k} \delta_{k+\theta} .
$$

\subsection{The Case of Indices $(d, d)$ where $d>1$}

Let $\mu$ be a Borel probability measure on $\mathbb{R}$, and let

$$
L^{2}(\mu):=L^{2}(\mathbb{R}, \mathscr{B}, \mu) .
$$

The notation $\operatorname{Prob}(\mathbb{R})$ will be used for these measures.

We saw that the restriction/extension problem for continuous positive definite (p.d.) functions $F$ on $\mathbb{R}$ may be translated into a spectral theoretic model in some 
$L^{2}(\mu)$ for suitable $\mu \in \operatorname{Prob}(\mathbb{R})$. We saw that extension from a finite open $(\neq \phi)$ interval leads to spectral representation in $L^{2}(\mu)$, and restrictions of

$$
\left(M_{\mu} f\right)(\lambda)=\lambda f(\lambda), f \in L^{2}(\mu)
$$

having deficiency-indices $(1,1)$; hence the case $d=1$.

Theorem 7.3.1. Fix $\mu \in \operatorname{Prob}(\mathbb{R})$. There is a 1-1 bijective correspondence between the following:

(1) certain closed subspaces $\mathscr{L} \subset L^{2}(\mu)$

(2) Hermitian restrictions $S_{\mathscr{L}}$ of $M_{\mu}$ (see (7.3.2)) such that

$$
D E F_{+}\left(S_{\mathscr{L}}\right)=\mathscr{L}
$$

The closed subspaces in (1) are specified as follows:

(i) $\operatorname{dim}(\mathscr{L})=d<\infty$

(ii) the following implication holds:

$$
g \neq 0, \text { and } g \in \mathscr{L} \Longrightarrow\left([\lambda \mapsto \lambda g(\lambda)] \notin L^{2}(\mu)\right)
$$

Then set

$$
\operatorname{dom}\left(S_{\mathscr{L}}\right):=\left\{f \in \operatorname{dom}\left(M_{\mu}\right) \mid \int \overline{g(\lambda)}(\lambda+i) f(\lambda) d \mu(\lambda), \forall g \in \mathscr{L}\right\}
$$

and set

$$
S_{\mathscr{L}}:=\left.M_{\mu}\right|_{\operatorname{dom}\left(S_{\mathscr{L}}\right)}
$$

where dom $\left(S_{\mathscr{L}}\right)$ is specified as in (7.3.5).

Proof. Note that the case $d=1$ is contained in the previous theorem.

Proof of $(1) \Rightarrow(2)$. We will be using an idea from [Jør81]. With assumptions (i)-(ii), in particular (7.3.4), one checks that $\operatorname{dom}\left(S_{\mathscr{L}}\right)$ as specified in $(7.3 .5)$ is dense in $L^{2}(\mu)$. In fact, the converse implication is also true.

Now setting $S_{\mathscr{L}}$ to be the restriction in (7.3.6), we conclude that

$$
S_{\mathscr{L}} \subseteq M_{\mu} \subseteq S_{\mathscr{L}}^{*}
$$

where

$$
\begin{aligned}
\operatorname{dom}\left(S_{\mathscr{L}}^{*}\right)= & \left\{h \in L^{2}(\mu) \mid \text { s.t. } \exists C<\infty\right. \text { and } \\
& \left|\int_{\mathbb{R}} \overline{h(\lambda)} \lambda f(\lambda) d \mu(\lambda)\right|^{2} \leq C \int_{\mathbb{R}}|f(\lambda)|^{2} d \mu(\lambda) \\
& \text { holds } \left.\forall f \in \operatorname{dom}\left(S_{\mathscr{L}}\right)\right\}
\end{aligned}
$$

The assertions in (2) now follow from this.

Proof of $(2) \Rightarrow(1)$. Assume that $S$ is a densely defined restriction of $M_{\mu}$, and let $D E F_{+}(S)=$ the $(+)$ deficiency space, i.e.,

$$
D E F_{+}(S)=\left\{g \in \operatorname{dom}\left(S^{*}\right) \mid S^{*} g=i g\right\}
$$

Assume $\operatorname{dim}\left(D E F_{+}(S)\right)=d$, and $1 \leq d<\infty$. Then set $\mathscr{L}:=D E F_{+}(S)$. Using [Jør81], one checks that (1) then holds for this closed subspace in $L^{2}(\mu)$. 
The fact that (7.3.4) holds for this subspace $\mathscr{L}$ follows from the observation:

$$
D E F_{+}(S) \cap \operatorname{dom}\left(M_{\mu}\right)=\{0\}
$$

for every densely defined restriction $S$ of $M_{\mu}$.

\subsection{Spectral Representation of Index $(1,1)$ Hermitian Operators}

In this section we give an explicit answer to the following question: How to go from any index $(1,1)$ Hermitian operator to a $\left(\mathscr{H}_{F}, D^{(F)}\right)$ model; i.e., from a given index $(1,1)$ Hermitian operator with dense domain in a separable Hilbert space $\mathscr{H}$, we build a p.d. continuous function $F$ on $\Omega-\Omega$, where $\Omega$ is a finite interval $(0, a)$, $a>0$.

So far, we have been concentrating on building transforms going in the other direction. But recall that, for a given continuous p.d. function $F$ on $\Omega-\Omega$, it is often difficult to answer the question of whether the corresponding operator $D^{(F)}$ in the RKHS $\mathscr{H}_{F}$ has deficiency indices $(1,1)$ or $(0,0)$.

Now this question answers itself once we have an explicit transform going in the opposite direction. Specifically, given any index $(1,1)$ Hermitian operator $S$ in a separable Hilbert space $\mathscr{H}$, we then to find a pair $(F, \Omega)$, p.d. function and interval, with the desired properties. There are two steps:

Step 1, writing down explicitly, a p.d. continuous function $F$ on $\Omega-\Omega$, and the associated RKHS $\mathscr{H}_{F}$ with operator $D^{(F)}$.

Step 2, constructing an intertwining isomorphism $W: \mathscr{H} \rightarrow \mathscr{H}_{F}$, having the following properties. $W$ will be an isometric isomorphism, intertwining the pair $(\mathscr{H}, S)$ with $\left(\mathscr{H}_{F}, D^{(F)}\right)$, i.e., satisfying $W S=D^{(F)} W$; and also intertwining the respective domains and deficiency spaces, in $\mathscr{H}$ and $\mathscr{H}_{F}$.

Moreover, starting with any $(1,1)$ Hermitian operator, we can even arrange a normalization for the p.d. function $F$ such that $\Omega=$ the interval $(0,1)$ will do the job.

We now turn to the details: where:

We will have three pairs $(\mathscr{H}, S),\left(L^{2}(\mathbb{R}, \mu)\right.$, restriction of $\left.M_{\mu}\right)$, and $\left(\mathscr{H}_{F}, D^{(F)}\right)$,

(i) $S$ is a fixed Hermitian operator with dense domain $\operatorname{dom}(S)$ in a separable Hilbert space $\mathscr{H}$, and with deficiency indices $(1,1)$.

(ii) From the given information in (i), we will construct a finite Borel measure $\mu$ on $\mathbb{R}$ such that an index- $(1,1)$ restriction of $M_{\mu}: f \mapsto \lambda f(\lambda)$ in $L^{2}(\mathbb{R}, \mu)$, is equivalent to $(\mathscr{H}, S)$.

(iii) Here $F:(-1,1) \rightarrow \mathbb{C}$ will be a p.d. continuous function, $\mathscr{H}_{F}$ the corresponding RKHS; and $D^{(F)}$ the usual operator with dense domain

$$
\begin{gathered}
\left\{F_{\varphi} \mid \varphi \in C_{c}^{\infty}(0,1)\right\} \text {, and } \\
D^{(F)}\left(F_{\varphi}\right)=\frac{1}{i} F_{\varphi^{\prime}}, \varphi^{\prime}=\frac{d \varphi}{d x} .
\end{gathered}
$$

We will accomplish the stated goal with the following system of intertwining operators: See Figure 7.4.1.

But we stress that, at the outset, only (i) is given; the rest $\left(\mu, F\right.$ and $\left.\mathscr{H}_{F}\right)$ will be constructed. Further, the solutions $(\mu, F)$ in Figure 7.4.1 are not unique; rather they depend on choice of selfadjoint extension in (i): Different selfadjoint 
extensions of $S$ in (i) yield different solutions $(\mu, F)$. But the selfadjoint extensions of $S$ in $\mathscr{H}$ are parameterized by von Neumann's theory; see e.g., [Rud73].

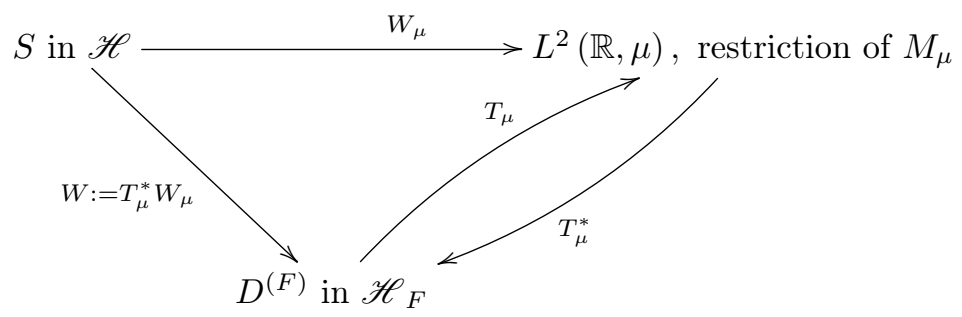

FIGURE 7.4.1. A system of intertwining operators.

REMARK 7.4.1. In our analysis of (i)-(iii), we may without loss of generality assume that the following normalizations hold:

$\left(z_{1}\right) \mu(\mathbb{R})=1$, so $\mu$ is a probability measure;

$\left(z_{2}\right) F(0)=1$, and the p.d. continuous solution

$\left(z_{3}\right) F:(-1,1) \rightarrow \mathbb{C}$ is defined on $(-1,1)$; so $\Omega:=(0,1)$.

Further, we may assume that the operator $S$ in $\mathscr{H}$ from (i) has simple spectrum.

TheOrem 7.4.2. Starting with $(\mathscr{H}, S)$ as in (i), there are solutions $(\mu, F)$ to (ii)-(iii), and intertwining operators $W_{\mu}, T_{\mu}$ as in Figure 7.4.1, such that

$$
W:=T_{\mu}^{*} W_{\mu}
$$

satisfies the intertwining properties for $(\mathscr{H}, S)$ and $\left(\mathscr{H}_{F}, D^{(F)}\right)$.

Proof. Since $S$ has indices $(1,1), \operatorname{dim} D E F_{ \pm}(S)=1$, and $S$ has selfadjoint extensions indexed by partial isometries $D E F_{+} \stackrel{v}{\longrightarrow} D E F_{-}$; see [Rud73, DS88]. We now pick $g_{+} \in D E F_{+},\left\|g_{+}\right\|=1$, and partial isometry $v$ with selfadjoint extension $S_{v}$, i.e.,

$$
S \subset S_{v} \subset S_{v}^{*} \subset S^{*}
$$

Hence $\left\{U_{v}(t) \mid t \in \mathbb{R}\right\}$ is a strongly continuous unitary representation of $\mathbb{R}$, acting in $\mathscr{H}, U_{v}(t):=e^{i t S_{v}}, t \in \mathbb{R}$. Let $P_{S_{v}}(\cdot)$ be the corresponding projection valued measure $(\mathrm{PVM})$ on $\mathscr{B}(\mathbb{R})$, i.e., we have

$$
U_{v}(t)=\int_{\mathbb{R}} e^{i t \lambda} P_{S_{v}}(d \lambda) ;
$$

and set

$$
d \mu(\lambda):=d \mu_{v}(\lambda)=\left\|P_{S_{v}}(d \lambda) g_{+}\right\|_{\mathscr{H}}^{2} .
$$

For $f \in L^{2}\left(\mathbb{R}, \mu_{v}\right)$, set

$$
W_{\mu_{v}}\left(f\left(S_{v}\right) g_{+}\right)=f
$$

then $W_{\mu_{v}}: \mathscr{H} \rightarrow L^{2}\left(\mathbb{R}, \mu_{v}\right)$ is isometric onto; and

$$
W_{\mu_{v}^{*}}(f)=f\left(S_{v}\right) g_{+},
$$


where

$$
f\left(S_{v}\right) g_{+}=\int_{\mathbb{R}} f(\lambda) P_{S_{v}}(d \lambda) g_{+} .
$$

For justification of these assertions, see e.g., [Nel69]. Moreover, $W_{\mu}$ has the intertwining properties sketched in Figure 7.4.1.

Returning to (7.4.4) and (iii) in the theorem, we now set $F=$ the restriction to $(-1,1)$ of

$$
\begin{array}{rlrl}
F_{\mu}(t) \quad & = & \left\langle g_{+}, U_{v}(t) g_{+}\right\rangle \\
= & & \left\langle g_{+}, \int_{\mathbb{R}} e^{i t \lambda} P_{S_{v}}(d \lambda) g_{+}\right\rangle \\
& = & & \int_{\mathbb{R}} e^{i t \lambda}\left\|P_{S_{v}}(d \lambda) g_{+}\right\|^{2} \\
& = & & \int_{\mathbb{R}} e^{i t \lambda} d \mu_{v}(\lambda) \\
\text { (by }(7.4 .5)) & & \widehat{d \mu_{v}}(t), \forall t \in \mathbb{R} . \\
& = & &
\end{array}
$$

We now show that

$$
F:=\left.F_{\mu}\right|_{(-1,1)}
$$

has the desired properties.

From Corollary 2.4.9, we have the isometry $T_{\mu}\left(F_{\varphi}\right)=\widehat{\varphi}, \varphi \in C_{c}(0,1)$, with adjoint

$$
\begin{gathered}
T_{\mu}^{*}(f)=(f d \mu)^{\vee}, \text { and } \\
\mathscr{H}_{F} \underset{T_{\mu}}{T_{\mu}^{*}} L^{2}(\mathbb{R}, \mu) ;
\end{gathered}
$$

see also Fig 7.4.1.

The following properties are easily checked:

$$
\begin{aligned}
& W_{\mu}\left(g_{+}\right)=\mathbf{1} \in L^{2}(\mathbb{R}, \mu), \text { and } \\
& T_{\mu}^{*}(\mathbf{1})=F_{0}=F(\cdot-0) \in \mathscr{H}_{F},
\end{aligned}
$$

as well as the intertwining properties stated in the theorem; see Fig. 7.4.1 for a summary.

Proof of (7.4.12) We will show instead that $W_{\mu}^{*}(\mathbf{1})=g_{+}$. From (7.4.8) we note that if $f \in L^{2}(\mathbb{R}, \mu)$ satisfies $f=\mathbf{1}$, then $f\left(S_{v}\right)=I=$ the identity operator in $\mathscr{H}$. Hence

which is $(7.4 .12)$.

$$
W_{\mu}^{*}(\mathbf{1}) \underset{(\text { by }(7.4 .7))}{=} \mathbf{1}\left(S_{v}\right) g_{+}=g_{+}
$$

Proof of (7.4.13) For $\varphi \in C_{c}(0,1)$ we have $\widehat{\varphi} \in L^{2}(\mathbb{R}, \mu)$, and

$$
\begin{array}{ccl}
T_{\mu}^{*} T_{\mu}\left(F_{\varphi}\right) & = & T_{\mu}^{*}(\widehat{\varphi}) \\
& = & (\widehat{\varphi} d \mu)^{\vee}=F_{\varphi} .
\end{array}
$$

Taking now an approximation $\left(\varphi_{n}\right) \subset C_{c}(0,1)$ to the Dirac unit mass at $x=0$, we get (7.4.13). 
Corollary 7.4.3. The deficiency indices of $D^{(F)}$ in $\mathscr{H}_{F}$ for $F(x)=e^{-|x|}$, $|x|<1$, are $(1,1)$.

Proof. Take $\mathscr{H}=L^{2}(\mathbb{R})=\left\{f\right.$ measurable on $\left.\left.\mathbb{R}\left|\int_{\mathbb{R}}\right| f(x)\right|^{2} d x<\infty\right\}$, where $d x=$ Lebesgue measure.

Take $g_{+}:=\left(\frac{1}{\lambda+i}\right)^{\vee}(x), x \in \mathbb{R} ;$ then $g_{+} \in L^{2}(\mathbb{R})=: \mathscr{H}$ since

$$
\begin{aligned}
\int_{\mathbb{R}}\left|g_{+}(x)\right|^{2} d x \quad \begin{array}{c}
\text { (Parseval) } \\
=
\end{array} & \int_{\mathbb{R}}\left|\frac{1}{\lambda+i}\right|^{2} d \lambda \\
= & \int_{\mathbb{R}} \frac{1}{1+\lambda^{2}} d \lambda=\pi .
\end{aligned}
$$

Now for $S$ and $S_{v}$ in Theorem 7.4.2, we take

$$
\begin{gathered}
S_{v} h=\frac{1}{i} \frac{d}{d x} h \text { on }\left\{h \in L^{2}(\mathbb{R}) \mid h^{\prime} \in L^{2}(\mathbb{R})\right\} \text { and } \\
S=S_{v} \mid\left\{h \mid h, h^{\prime} \in L^{2}(\mathbb{R}), h(0)=0\right\},
\end{gathered}
$$

then by $[\mathbf{J} \varnothing \mathbf{r} \mathbf{8 1}]$, we know that $S$ is an index $(1,1)$ operator, and that $g_{+} \in$ $D E F_{+}(S)$. The corresponding p.d. continuous function $F$ is the restriction to $|t|<1$ of the p.d. function:

$$
\begin{aligned}
\left\langle g_{+}, U_{v}(t) g_{+}\right\rangle_{\mathscr{H}} & =\int_{\mathbb{R}} \frac{1}{\lambda-i} \frac{e^{i t \lambda}}{\lambda+i} d \lambda \\
& =\left(\frac{1}{1+\lambda^{2}}\right)^{\vee}(t)=\pi e^{-|t|}
\end{aligned}
$$

ExAmPLE 7.4.4 (Lévy-measures). Let $0<\alpha \leq 2,-1<\beta<1, v>0$; then the Lévy-measures $\mu$ on $\mathbb{R}$ are indexed by $(\alpha, \beta, \nu)$, so $\mu=\mu_{(\alpha, \beta, \nu)}$. They are absolutely continuous with respect to Lebesgue measure $d \lambda$ on $\mathbb{R}$; and for $\alpha=1$,

$$
F_{(\alpha, \beta, \nu)}(x)=\widehat{\mu_{(\alpha, \beta, \nu)}}(x), x \in \mathbb{R},
$$

satisfies

$$
F_{(\alpha, \beta, \nu)}(x)=\exp \left(-\nu|x| \cdot\left(1+\frac{2 i \beta}{\pi}-\operatorname{sgn}(x) \ln |x|\right)\right) .
$$

The case $\alpha=2, \beta=0$, reduces to the Gaussian distribution.

The measures $\mu_{(1, \beta, \nu)}$ have infinite variance, i.e.,

$$
\int_{\mathbb{R}} \lambda^{2} d \mu_{(1, \beta, \nu)}=\infty
$$

As a Corollary of Theorem 7.4.2, we therefore conclude that, for the restrictions,

$$
F_{(1, \beta, \nu)}^{(\text {res })}(x)=F_{(1, \beta, \nu)}(x), \text { in } x \in(-1,1),(\text { see }(7.4 .15)-(7.4 .16))
$$

the associated Hermitian operator $D^{F^{(r e s)}}$ all have deficiency indices $(1,1)$.

In connection Levy measures, see e.g., [ST94]. 


\section{CHAPTER 8}

\section{Open Questions}

\subsection{From Restriction Operator to Restriction of p.d. Function}

QUESTION 8.1.1. How to write a formula for the restriction of a suitable positive definite (p.d.) function associated to a given $(1,1)$ operator?

In section 7.4 , we create a model for $(1,1)$ operators $S$ with simple spectrum; and a choice of cyclic (defect) vector $v_{0}$. Hence $S$ is the restriction of a selfadjoint operator $T$.

From $T$ (simple spectrum) and the vector $v_{0}$, we get a Borel probability measure $\mu$ on $\mathbb{R}$; and by Bochner, or Stone, we get the corresponding continuous p.d. function $F$ on $\mathbb{R}$. It would be nice to have a formula for the particular restriction of $F$ which corresponds to the $(1,1)$ operator $S$ in the model.

The main difference between our measures on $\mathbb{R}$ and the measures used in fractional Brownian motion and related processes is that our measures are finite on $\mathbb{R}$, but the others aren't; instead they are what is called tempered. See [AL08].

This means that they are unbounded but only with a polynomial degree. For example, Lebesgue measure $d x$ on $\mathbb{R}$ is tempered. Suppose $1 / 2<g<1$, then the positive measure $d \mu(x)=|x|^{g} d x$ is tempered, and this measure is what is used in accounting for the p.d. functions discussed in the papers on Fractional Brownian motion.

But we could ask the same kind of questions for tempered measures as we do for the finite positive measures.

With this analogy, $[\mathbf{J} \varnothing \mathbf{r} \mathbf{8 1}]$ was about $(1,1)$ operators from Lebesgue measure $d x$ on $\mathbb{R}$. PJ also looked at $(m, m)$ operators, but only from Lebesgue measure.

$$
\text { 8.2. The Splitting } \mathscr{H}_{F}=\mathscr{H}_{F}^{(\text {atom })} \oplus \mathscr{H}_{F}^{(a c)} \oplus \mathscr{H}_{F}^{(\text {sing })}
$$

Let $\Omega$ be as usual, connected and open in $\mathbb{R}^{n}$; and let $F$ be a p.d. continuous function on $\Omega^{-1} \Omega$. We then pass to the RKHS $\mathscr{H}_{F}$. Suppose $\operatorname{Ext}(F)$ is non-empty. We then get a unitary representation $U$ of $G=\mathbb{R}^{n}$, acting on $\mathscr{H}_{F}$; and its associated PVM $P_{U}$. This gives rise to an orthogonal splitting of $\mathscr{H}_{F}$ into three parts, atomic, absolutely continuous, and continuous singular, defined from $P_{U}$.

QUESTION 8.2.1. What are some interesting examples illustrating the triple splitting of $\mathscr{H}_{F}$, as in section 2.5, eq. (2.5.6)?

It will be nice to see some examples where we have more than one non-zero subspaces; so an example of $F$ be a p.d. continuous function on $\Omega^{-1} \Omega$ where there is a mixture of the three possibilities, atomic, absolutely continuous, and continuous singular. So we would like an example of a $(F, \Omega)$, such that $F$ be a p.d. continuous function on $\Omega^{-1} \Omega$, has its $\mathscr{H}_{F}$ splitting with more than one of the two non-zero. 
So fix $(F, \Omega)$; and it would be interesting to study cases where the same $(F, \Omega)$ has two of its three subspaces non-zero? Or all three non-zero? The examples of $(F, \Omega)$ fall in one of the three boxes only. Can we get spectrum in all three boxes, or at least not deposit everything in just one of the three?

In section 2.5, we constructed an interesting example for the splitting of the RKHS $\mathscr{H}_{F}$ : All three subspaces $\mathscr{H}_{F}^{(\text {atom })}, \mathscr{H}_{F}^{(a c)}$, and $\mathscr{H}_{F}^{(\text {sing })}$ are non-zero; the first one is one-dimensional, and the other two are infinite-dimensional. See Example 2.5.2 for details.

\subsection{The Case of $G=\mathbb{R}^{1}$}

We can move to the circle group $\mathbb{T}=\mathbb{R} / \mathbb{Z}$, and we already started that. Or we can stay with $\mathbb{R}$, or go to $\mathbb{R}^{n}$; or go to Lie groups (if Lie groups we must also look at the universal simply connected covering groups.) All very interesting questions.

A FEW POINTS, about a single interval and asking for p.d. extensions to $\mathbb{R}$ :

(1) The case of $\mathbb{R}$, we are asking to extend a (small) p.d. continuous function $F$ from an interval to all of $\mathbb{R}$, so that the extension to $\mathbb{R}$ is also p.d. continuous; - in this case we always have existence. This initial interval may even be "very small." If we have several intervals, existence may fail, but the problem is interesting. We have calculated a few examples, but nothing close to a classification! Here is what we know about extension from a single interval:

(2) In all cases, we have existence, but the p.d continuous extension to $\mathbb{R}$ may or may not be unique.

(3) To decide the uniqueness, we turn to the RKHS $\mathscr{H}_{F}$ (remember now we are on the interval), and we calculate deficiency indices there, but we are in RKHS $\mathscr{H}_{F}$. They may be $(0,0)$ or $(1,1)$.

- In the first case, we have uniqueness; in the second case not.

- Both cases are very interesting; also indices $(0,0)$.

By contrast, in our earlier papers, e.g., on momentum operators, we never looked at RKHSs related to this other $\mathscr{H}_{F}$ we now study.

(4) Actually, computable ONBs in $\mathscr{H}_{F}$ would be very interesting. So here is a fun project:

We can use what we know about selfadjoint extensions of operators with deficiency indices $(1,1)$ to try to classify all the p.d continuous extension to $\mathbb{R}$. But it is not trivial; remember the interplay between operator extensions and p.d. extensions is subtle. While both are "extension" problems, the interplay between them is subtle and non-intuitive.

WARNING: This new classification question probably has nothing to do with questions for the moment operator in its various incarnations, see e.g., [JPT12b, JPT12a] and [PT13].

- This is because there are lots of other operators with deficiency indices $(1,1)$, having nothing to do at all with moment operators.

- From examples we calculated, so far, for the relevant operators with deficiency indices $(1,1)$ in RKHS $\mathscr{H}_{F}$ (remember now we are on the interval), are totally unrelated to moment operators.

- With $\mathscr{H}_{F}$, you are aiming to understand deficiency index-operators in the case of $(1,1)$, but in the abstract; not just an example. 
There are two sources of earlier papers; one is M. Krein, and the other L. deBranges. Both are covered beautifully in a book by Dym and McKean; it is now in the Dover series.

\subsection{The Extreme Points of $\operatorname{Ext}(F)$ and $\Im\{F\}$}

Given a locally defined p.d. function $F$, i.e., a p.d. function $F$ defined on a proper subset in a group, then the real part $\Re\{F\}$ is also p.d.. Can anything be said about the imaginary part, $\Im\{F\}$ ? See section 5.4.

Assuming that $F$ is also continuous, then what are the extreme points in the compact convex set $\operatorname{Ext}(F)$, i.e., what is the subset $\operatorname{ext}(\operatorname{Ext}(F))$ ? How do properties of $\operatorname{Ext}(F)$ depend on the imaginary part, i.e., on the function $\Im\{F\}$ ? How do properties of the skew-Hermitian operator $D^{(F)}$ (in the RKHS $\mathscr{H}_{F}$ ) depend on the imaginary part, i.e., on the function $\Im\{F\}$ ? 


\section{List of Figures}

2.3.1 The approximate identity $\varphi_{n, x}(\cdot) \quad 18$

$\begin{array}{ll}\text { 2.3.2 Two versions of } \Omega=(0, a) \subset \mathbb{T}^{1} & 19\end{array}$

2.5.1 The measure $d \mu(\lambda)$ in example 2.5.2. 29

2.5.2 Cumulative distribution $=\int_{-\infty}^{\lambda} d \mu(\lambda)$, as in example 2.5.2. $\quad 30$

2.9.1 Functions on $\mathbb{T}=\mathbb{R} / \mathbb{Z} \longleftrightarrow(1$-periodic functions on $\mathbb{R})$. 36

2.9.2 $\tilde{F}_{\text {per }}(t)>F(t)$ on $\left[-\frac{1}{2}, \frac{1}{2}\right] \quad 36$

3.1.1 Spline extension of $F:(-a, a) \rightarrow \mathbb{R} \quad 45$

3.1.2 An example of Polya extension of $F$ on $(-a, a) . \quad 46$

3.1.3 Extension of $F_{1}(x)=\frac{1}{1+x^{2}} ; \Omega=(0,1) \quad 46$

3.1.4 Extension of $F_{2}(x)=1-|x| ; \Omega=\left(0, \frac{1}{2}\right) \quad 47$

3.1.5 Extension of $F_{3}(x)=e^{-|x|} ; \Omega=(0,1) \quad 47$

3.1.6 Extension of $F_{4}(x)=\left(\frac{\sin \pi x}{\pi x}\right)^{2} ; \Omega=\left(0, \frac{1}{2}\right) \quad 47$

3.1.7 Extension $F_{5}(x)=e^{-x^{2} / 2} ; \Omega=(0,1) \quad 47$

3.1.8 Extension of $F_{6}(x)=\cos (x) ; \Omega=\left(0, \frac{\pi}{4}\right) \quad 49$

3.1.9 $\operatorname{dim}(\operatorname{RKHS}(\cos x$ on $\mathbb{R}))=2$; but RKHS(Polya ext. to $\mathbb{R})$ is $\infty$-dimensional. $\quad 51$

3.2.1 A Polya (spline) extension of $F_{3}=e^{-|x|} ; \Omega=(-1,1) . \quad 53$

4.3.1 The kernel $F_{x}$ and its derivative (the case of $F_{2}$ ) 85

4.3.2 The covariance function $E_{x}(\cdot)=\min (x, \cdot)$ of Brownian motion. $\quad 86$

4.3.3 Monte-Carlo simulation of Brownian motion starting at $x=0$, with 5 sample paths.

4.3.4 Monte-Carlo simulation of Brownian bridge pinned at $(0,0)$ and $(1,1)$, with 5 sample paths.

4.3.5 $F_{p}(x)=1-|x|^{p}, x \in\left(-\frac{1}{2}, \frac{1}{2}\right)$ and $0<p \leq 1 . \quad 91$

4.3.6 The integral kernel $K_{\frac{1}{2}}(x, y)=|x| \wedge|y|$. 92

4.3.7 Monte-Carlo simulation of Ornstein-Uhlenbeck process with 5 sample paths. $\beta=\gamma=1, v_{0}=1, t \in[0,5]$

5.1.1 The examples of $F_{2}$ and $F_{3}$. 
7.1.1 The measures $\mu_{i} \in \operatorname{Ext}\left(F_{i}\right)$ extending p.d. functions $F_{i}$ in Table 3.1.1, $i=1,2, \ldots 5$.

7.4.1 A system of intertwining operators. 


\section{List of Tables}

3.1.1 The deficiency indices of $D^{(F)}: F_{\varphi} \mapsto F_{\varphi^{\prime}}$ in examples 3.1.3-3.1.9 52

3.1.2 Type II extensions. Six cases of p.d. continuous functions $F_{i}$ defined on $\begin{array}{ll}\text { a finite interval }(-a, a) . & 52\end{array}$

3.1.3 The canonical isometric embeddings: $\mathscr{H}_{F_{i}} \hookrightarrow L^{2}\left(\mathbb{R}, d \mu_{i}\right), i=1, \ldots, 6 . \quad 53$

4.1.1 Boundary values of the Shannon functions, s.t. $\Re\left\{f_{n}\right\}(1)=\Re\left\{f_{n}\right\}(0)$, and $\Im\left\{f_{n}\right\}(1)=-\Im\left\{f_{n}\right\}(0) . \quad 63$

4.1.2 Application of Proposition 4.1.42 to Table 3.1.1. 74

4.4.1 An overview of two cases: $F_{2}$ v.s. $F_{3}$. 95

7.1.1 Application of Theorem 7.1.10 to Table 3.1.1. 115 


\section{List of Symbols}

The reproducing kernel Hilbert space (RKHS) of $F$.

$D E F$

The derivative operator $F_{\varphi} \mapsto F_{\frac{d \varphi}{d x}}$

$\operatorname{Ext}(F)$

The deficiency space of $D^{(F)}$ acting in the Hilbert space $\mathscr{H}_{F}$.

Set of unitary representations of $G$ on a Hilbert space $\mathscr{K}$, i.e., the triple $\left(U, \mathscr{K}, k_{0}\right)$, that extends $F$.

$\operatorname{Ext}_{1}(F) \quad$ The triples in $\operatorname{Ext}(F)$, where the representation space is $\mathscr{H}_{F}$, and so the extension of $F$ is realized on $\mathscr{H}_{F}$.

$\operatorname{Ext}_{2}(F) \quad \operatorname{Ext}(F) \backslash \operatorname{Ext}_{1}(F)$, i.e., the extension is realized on an enlargement Hilbert space.

$F_{\varphi} \quad$ The convolution of $F$ and $\varphi$, where $\varphi \in C_{c}(\Omega)$.

$T_{F} \quad$ Mercer operator associated to $F$.
} 


\section{Index}

absolutely continuous, 14, 23, 25, 36, 106, 123,124

approximate identity, 11, 37, 95

atom, 25, 38, 51, 118, 124

Bessel frame, 67

Bochner, 6, 11, 27, 104

Brownian bridge, 85

Brownian motion, 56, 80, 84, 85

complex exponential, 38, 39

covariance, $5,56,58,86$

deficiency indices, 4, 7, 14, 35, 47, 48, 50, $70,80,98,105,107,110,114$

extension problem, 3, 4, 7, 28, 118

Fourier duality, 4, 73

fractional Brownian motion, 90

Friedrichs extension, 59, 82, 92

Gaussian processes, 5, 80

GNS, 24, 29, 49

group

character, 24

circle, 31, 108

covering, 4,30

dual, 21

Lie, 3, 95

locally compact abelian, 21, 108

non-Abelian, 4

non-commutative, 4

simply connected, 4,30

intertwining, 10, 41, 117, 120, 121

isometry, 10, 28, 41, 42, 49, 100, 112, 122

Kolmogorov, 4, 5

Krein, 4, 40, 126

measure

atomic, 118

Bernoulli, 48

Borel, 14, 99, 120

boundary, 64, 89, 95
Cantor, 27

convolution, 108

Dirac, 15, 27

fractal, 48, 108

Haar, 21, 29, 62, 63, 94

Lévy, 123

Lebesgue, 25, 47

positive, 99

probability, 5, 22, 35, 36, 110, 112, 114,

121

product, 108

projection-valued, 21

PVM, 25, 74, 110, 116, 121

singular, 25

tempered, 124

operator

bounded, 23, 96

closed, 68

Mercer, 51, 54, 82, 92, 93

partial differential, 93

selfadjoint, 3, 59, 68, 74, 93

semibounded, 59, 92

skew-Hermitian, 3, 13, 29, 38, 47, 55, 69, $80,98,126$

unbounded, 4, 50, 68, 92

unitary one-parameter group, 14, 20, 112

Ornstein-Uhlenbeck, 5, 45, 80, 91

orthogonal, 25, 54, 124

Paley-Wiener, 28, 63

perturbation, 56, 58, 89

Poincaré-inequality, 66

polar decomposition, 69

Polya, 14, 43

positive definite, $3,5-9,12,13,17,21,28$, $32,33,35,39,43,46,56,74,80,94$, 95, 99, 100, 118

distribution, 75

projection, 56, 65, 97

Radon-Nikodym, 14, 100, 103

Renormalization, 64 
RKHS, 3, 7-10, 13, 15, 21, 25, 32, 36, 43,

$47,48,77,80,94,97,100$

Schöenberg, 5, 99

Schwartz

distribution, 44,51

test function, 74

selfadjoint extension, $31,38,56,74,93$, 115,121

Shannon sampling, 45, 60

spectrum, 31, 38, 110, 116, 124

spline, 5,43

stochastic processes, $6,85,91$

type $1,10,49$

type $2,10,49$

unitary dilations, 12

unitary representation, 4, 12, 13, 24, 29, 48, 110

von Neumann, 4, 38, 121 


\section{Bibliography}

[ABDdS93] Daniel Alpay, Vladimir Bolotnikov, Aad Dijksma, and Henk de Snoo. On some operator colligations and associated reproducing kernel Hilbert spaces. In Operator extensions, interpolation of functions and related topics (Timişoara, 1992), volume 61 of Oper. Theory Adv. Appl., pages 1-27. Birkhäuser, Basel, 1993.

[ACF09] Inés Armendáriz, Antonio Cuevas, and Ricardo Fraiman. Nonparametric estimation of boundary measures and related functionals: asymptotic results. Adv. in Appl. Probab., 41(2):311-322, 2009.

[AD86] Daniel Alpay and Harry Dym. On applications of reproducing kernel spaces to the Schur algorithm and rational $J$ unitary factorization. In I. Schur methods in operator theory and signal processing, volume 18 of Oper. Theory Adv. Appl., pages 89-159. Birkhäuser, Basel, 1986.

$\left[\mathrm{ADL}^{+} 10\right]$ Daniel Alpay, Aad Dijksma, Heinz Langer, Simeon Reich, and David Shoikhet. Boundary interpolation and rigidity for generalized Nevanlinna functions. Math. Nachr., 283(3):335-364, 2010.

[AG93] N. I. Akhiezer and I. M. Glazman. Theory of linear operators in Hilbert space. Dover Publications Inc., New York, 1993. Translated from the Russian and with a preface by Merlynd Nestell, Reprint of the 1961 and 1963 translations, Two volumes bound as one.

[AH13] Giles Auchmuty and Qi Han. Spectral representations of solutions of linear elliptic equations on exterior regions. J. Math. Anal. Appl., 398(1):1-10, 2013.

[AJ12] Daniel Alpay and Palle E. T. Jorgensen. Stochastic processes induced by singular operators. Numer. Funct. Anal. Optim., 33(7-9):708-735, 2012.

[AJL11] Daniel Alpay, Palle Jorgensen, and David Levanony. A class of Gaussian processes with fractional spectral measures. J. Funct. Anal., 261(2):507-541, 2011.

[AJSV13] Daniel Alpay, Palle Jorgensen, Ron Seager, and Dan Volok. On discrete analytic functions: products, rational functions and reproducing kernels. J. Appl. Math. Comput., 41(1-2):393-426, 2013.

[AL08] Daniel Alpay and David Levanony. On the reproducing kernel Hilbert spaces associated with the fractional and bi-fractional Brownian motions. Potential Anal., 28(2):163-184, 2008.

[AL10] Daniel Alpay and David Levanony. Linear stochastic systems: a white noise approach. Acta Appl. Math., 110(2):545-572, 2010.

[Alp92] Daniel Alpay. On linear combinations of positive functions, associated reproducing kernel spaces and a non-Hermitian Schur algorithm. Arch. Math. (Basel), 58(2):174182, 1992.

[Aro50] N. Aronszajn. Theory of reproducing kernels. Trans. Amer. Math. Soc., 68:337-404, 1950.

[ARR13] Luigi Accardi, Habib Rebei, and Anis Riahi. The quantum decomposition of random variables without moments. Infin. Dimens. Anal. Quantum Probab. Relat. Top., 16(2):1350012, 28, 2013.

[Arv86] William Arveson. Markov operators and OS-positive processes. J. Funct. Anal., 66(2):173-234, 1986.

[Aur11] Frank Aurzada. On the one-sided exit problem for fractional Brownian motion. Electron. Commun. Probab., 16:392-404, 2011.

[Bat90] C. J. K. Batty. Vector-valued Choquet theory and transference of boundary measures. Proc. London Math. Soc. (3), 60(3):530-548, 1990. 
[BCR84] Christian Berg, Jens Peter Reus Christensen, and Paul Ressel. Harmonic analysis on semigroups, volume 100 of Graduate Texts in Mathematics. Springer-Verlag, New York, 1984. Theory of positive definite and related functions.

[BM13] John P. Boyd and Philip W. McCauley. Quartic Gaussian and inverse-quartic Gaussian radial basis functions: the importance of a nonnegative Fourier transform. Comput. Math. Appl., 65(1):75-88, 2013.

[CM13] B. Currey and A. Mayeli. The Orthonormal Dilation Property for Abstract Parseval Wavelet Frames. Canad. Math. Bull., 56(4):729-736, 2013.

[Cór89] A. Córdoba. Dirac combs. Lett. Math. Phys., 17(3):191-196, 1989.

[CRKS79] P. Cotta-Ramusino, W. Krüger, and R. Schrader. Quantum scattering by external metrics and Yang-Mills potentials. Ann. Inst. H. Poincaré Sect. A (N.S.), 31(1):43$71,1979$.

[dB68] Louis de Branges. Hilbert spaces of entire functions. Prentice-Hall Inc., Englewood Cliffs, N.J., 1968.

[dBR66] Louis de Branges and James Rovnyak. Canonical models in quantum scattering theory. In Perturbation Theory and its Applications in Quantum Mechanics (Proc. Adv. Sem. Math. Res. Center, U.S. Army, Theoret. Chem. Inst., Univ. of Wisconsin, Madison, Wis., 1965), pages 295-392. Wiley, New York, 1966.

[Dev59] Allen Devinatz. On the extensions of positive definite functions. Acta Math., 102:109134, 1959.

[Dev72] Allen Devinatz. The deficiency index of a certain class of ordinary self-adjoint differential operators. Advances in Math., 8:434-473, 1972.

[DJ10] Dorin Ervin Dutkay and Palle E. T. Jorgensen. Spectral theory for discrete Laplacians. Complex Anal. Oper. Theory, 4(1):1-38, 2010.

[DJ12] Dorin Ervin Dutkay and Palle E. T. Jorgensen. Fourier duality for fractal measures with affine scales. Math. Comp., 81(280):2253-2273, 2012.

[DRSS94] R. Del Rio, B. Simon, and G. Stolz. Stability of spectral types for Sturm-Liouville operators. Math. Res. Lett., 1(4):437-450, 1994.

[DS88] Nelson Dunford and Jacob T. Schwartz. Linear operators. Part II. Wiley Classics Library. John Wiley \& Sons Inc., New York, 1988. Spectral theory. Selfadjoint operators in Hilbert space, With the assistance of William G. Bade and Robert G. Bartle, Reprint of the 1963 original, A Wiley-Interscience Publication.

[Fal74] B. J. Falkowski. Factorizable and infinitely divisible PUA representations of locally compact groups. J. Mathematical Phys., 15:1060-1066, 1974.

[Fan10] Mark Fannes. An introduction to quantum probability. In Theoretical foundations of quantum information processing and communication, volume 787 of Lecture Notes in Phys., pages 1-38. Springer, Berlin, 2010.

[FM13] J. C. Ferreira and V. A. Menegatto. Positive definiteness, reproducing kernel Hilbert spaces and beyond. Ann. Funct. Anal., 4(1):64-88, 2013.

[FR42] W. H. J. Fuchs and W. W. Rogosinski. A note on Mercer's theorem. J. London Math. Soc., 17:204-210, 1942.

[Fug74] Bent Fuglede. Boundary minimum principles in potential theory. Math. Ann., 210:213-226, 1974.

[GSS83] T. N. E. Greville, I. J. Schoenberg, and A. Sharma. The behavior of the exponential Euler spline $S_{n}(x ; t)$ as $n \rightarrow \infty$ for negative values of the base $t$. In Second Edmonton conference on approximation theory (Edmonton, Alta., 1982), volume 3 of CMS Conf. Proc., pages 185-198. Amer. Math. Soc., Providence, RI, 1983.

[Hid80] Takeyuki Hida. Brownian motion, volume 11 of Applications of Mathematics. Springer-Verlag, New York, 1980. Translated from the Japanese by the author and T. P. Speed.

[HS12] Ze-Chun $\mathrm{Hu}$ and Wei Sun. Balayage of semi-Dirichlet forms. Canad. J. Math., 64(4):869-891, 2012.

[IM65] Kiyoshi Itô and Henry P. McKean, Jr. Diffusion processes and their sample paths. Die Grundlehren der Mathematischen Wissenschaften, Band 125. Academic Press Inc., Publishers, New York, 1965.

[Ion01] Eugen J. Ionascu. Rank-one perturbations of diagonal operators. Integral Equations Operator Theory, 39(4):421-440, 2001. 
[IW89] Nobuyuki Ikeda and Shinzo Watanabe. Stochastic differential equations and diffusion processes, volume 24 of North-Holland Mathematical Library. North-Holland Publishing Co., Amsterdam, second edition, 1989.

[JKS08] Palle E. T. Jorgensen, Keri Kornelson, and Karen Shuman. Orthogonal exponentials for Bernoulli iterated function systems. In Representations, wavelets, and frames, Appl. Numer. Harmon. Anal., pages 217-237. Birkhäuser Boston, Boston, MA, 2008.

[JKS11] Palle E. T. Jorgensen, Keri A. Kornelson, and Karen L. Shuman. Iterated function systems, moments, and transformations of infinite matrices. Mem. Amer. Math. Soc., 213(1003):x+105, 2011.

[JKS12] Palle E. T. Jorgensen, Keri A. Kornelson, and Karen L. Shuman. An operator-fractal. Numer. Funct. Anal. Optim., 33(7-9):1070-1094, 2012.

[JLW69] Arthur M. Jaffe, Oscar E. Lanford, III., and Arthur S. Wightman. A general class of cut-off model field theories. Comm. Math. Phys., 15:47-68, 1969.

[JÓ98] Palle E. T. Jorgensen and Gestur Ólafsson. Unitary representations of Lie groups with reflection symmetry. J. Funct. Anal., 158(1):26-88, 1998.

[JÓ00] Palle E. T. Jorgensen and Gestur Ólafsson. Unitary representations and OsterwalderSchrader duality. In The mathematical legacy of Harish-Chandra (Baltimore, MD, 1998), volume 68 of Proc. Sympos. Pure Math., pages 333-401. Amer. Math. Soc., Providence, RI, 2000.

[Jør81] Palle E. T. Jørgensen. A uniqueness theorem for the Heisenberg-Weyl commutation relations with nonselfadjoint position operator. Amer. J. Math., 103(2):273-287, 1981.

[Jor86] Palle E. T. Jorgensen. Analytic continuation of local representations of Lie groups. Pacific J. Math., 125(2):397-408, 1986.

[Jor87] Palle E. T. Jorgensen. Analytic continuation of local representations of symmetric spaces. J. Funct. Anal., 70(2):304-322, 1987.

[Jor89] Palle E. T. Jorgensen. Positive definite functions on the Heisenberg group. Math. Z., 201(4):455-476, 1989.

[Jor90] Palle E. T. Jorgensen. Extensions of positive definite integral kernels on the Heisenberg group. J. Funct. Anal., 92(2):474-508, 1990.

[Jor91a] Palle E. T. Jorgensen. Integral representations for locally defined positive definite functions on Lie groups. Internat. J. Math., 2(3):257-286, 1991.

[Jor91b] Palle E. T. Jorgensen. Integral representations for locally defined positive definite functions on Lie groups. Internat. J. Math., 2(3):257-286, 1991.

[Jor07] Palle E. T. Jorgensen. The measure of a measurement. J. Math. Phys., 48(10):103506, 15, 2007.

[JP12] P. E. T. Jorgensen and A. M. Paolucci. $q$-frames and Bessel functions. Numer. Funct. Anal. Optim., 33(7-9):1063-1069, 2012.

[JP13a] Palle E. T. Jorgensen and Erin P. J. Pearse. A discrete Gauss-Green identity for unbounded Laplace operators, and the transience of random walks. Israel J. Math., 196(1):113-160, 2013.

[JP13b] Palle E. T. Jorgensen and Erin P. J. Pearse. A discrete Gauss-Green identity for unbounded Laplace operators, and the transience of random walks. Israel J. Math., 196(1):113-160, 2013.

[JP13c] Palle E. T. Jorgensen and Erin P. J. Pearse. Multiplication operators on the energy space. J. Operator Theory, 69(1):135-159, 2013.

[JPT12a] Palle Jorgensen, Steen Pedersen, and Feng Tian. Translation representations and scattering by two intervals. J. Math. Phys., 53(5):053505, 49, 2012.

[JPT12b] Palle E.T. Jorgensen, Steen Pedersen, and Feng Tian. Momentum operators in two intervals: Spectra and phase transition. Complex Analysis and Operator Theory, 2012.

[KL14] Mark G. Krein and Heinz Langer. Continuation of Hermitian Positive Definite Functions and Related Questions. Integral Equations Operator Theory, 78(1):1-69, 2014.

[Kre46] M. Krein. Concerning the resolvents of an Hermitian operator with the deficiencyindex $(m, m)$. C. R. (Doklady) Acad. Sci. URSS (N.S.), 52:651-654, 1946.

[KS02] Vadim Kostrykin and Robert Schrader. Statistical ensembles and density of states. In Mathematical results in quantum mechanics (Taxco, 2001), volume 307 of Contemp. Math., pages 177-208. Amer. Math. Soc., Providence, RI, 2002. 
[KT09] Andi Kivinukk and Gert Tamberg. Interpolating generalized Shannon sampling operators, their norms and approximation properties. Sampl. Theory Signal Image Process., 8(1):77-95, 2009.

[Lit99] A. A. Litvinyuk. On types of distributions for sums of a class of random power series with independent identically distributed coefficients. Ukraïn. Mat. Zh., 51(1):128132, 1999.

[LP85] Peter D. Lax and Ralph S. Phillips. Translation representations for automorphic solutions of the wave equation in non-Euclidean spaces; the case of finite volume. Trans. Amer. Math. Soc., 289(2):715-735, 1985.

[LP89] Peter D. Lax and Ralph S. Phillips. Scattering theory, volume 26 of Pure and Applied Mathematics. Academic Press Inc., Boston, MA, second edition, 1989. With appendices by Cathleen S. Morawetz and Georg Schmidt.

[Maa10] H. Maassen. Quantum probability and quantum information theory. In Quantum information, computation and cryptography, volume 808 of Lecture Notes in Phys., pages 65-108. Springer, Berlin, 2010.

[Maz11] Vladimir Maz'ya. Sobolev spaces with applications to elliptic partial differential equations, volume 342 of Grundlehren der Mathematischen Wissenschaften /Fundamental Principles of Mathematical Sciences]. Springer, Heidelberg, augmented edition, 2011.

[Mov12] Yevgenya Movshovich. Inferior mean of Cantor boundary measures. J. Math. Anal. Appl., 386(2):780-795, 2012.

[Muh74] Paul S. Muhly. Some remarks on the spectra of unitary dilations. Studia Math., 49:139-147, 1973/74.

[Nel57] Edward Nelson. Kernel functions and eigenfunction expansions. Duke Math. J., $25: 15-27,1957$.

[Nel69] Edward Nelson. Topics in dynamics. I: Flows. Mathematical Notes. Princeton University Press, Princeton, N.J., 1969.

[Neu13] J. Neunhäuserer. Absolutely continuous random power series in reciprocals of Pisot numbers. Statist. Probab. Lett., 83(2):431-435, 2013.

[Nus75] A. Edward Nussbaum. Extension of positive definite functions and representation of functions in terms of spherical functions in symmetric spaces of noncompact type of rank 1. Math. Ann., 215:97-116, 1975.

[OH13] Anatol Odzijewicz and Maciej Horowski. Positive kernels and quantization. J. Geom. Phys., 63:80-98, 2013.

[OS73] Konrad Osterwalder and Robert Schrader. Axioms for Euclidean Green's functions. Comm. Math. Phys., 31:83-112, 1973.

[Par09] K. R. Parthasarathy. An invitation to quantum information theory. In Perspectives in mathematical sciences. I, volume 7 of Stat. Sci. Interdiscip. Res., pages 225-245. World Sci. Publ., Hackensack, NJ, 2009.

[Pól49] George Pólya. Remarks on characteristic functions. In Proc. First Berkeley Conf. on Math. Stat. and Prob, pages 115-123, 1949.

[PS75] K. R. Parthasarathy and K. Schmidt. Stable positive definite functions. Trans. Amer. Math. Soc., 203:161-174, 1975.

[PT13] Steen Pedersen and Feng Tian. Momentum operators in the unit square. Integral Equations Operator Theory, 77(1):57-88, 2013.

[Ric03] Christoph Richard. Dense Dirac combs in Euclidean space with pure point diffraction. J. Math. Phys., 44(10):4436-4449, 2003.

[RSN56] Friedrich Riesz and Béla Sz.-Nagy. Vorlesungen über Funktionalanalysis. Hochschulbücher für Mathematik, Bd. 27. VEB Deutscher Verlag der Wissenschaften, Berlin, 1956. Translation by Siegfried Brehmer and Brigitte Mai,.

[Rud63] Walter Rudin. The extension problem for positive-definite functions. Illinois J. Math., 7:532-539, 1963.

[Rud70] Walter Rudin. An extension theorem for positive-definite functions. Duke Math. J., 37:49-53, 1970.

[Rud73] Walter Rudin. Functional analysis. McGraw-Hill Book Co., New York, 1973. McGraw-Hill Series in Higher Mathematics.

[Rud90] Walter Rudin. Fourier analysis on groups. Wiley Classics Library. John Wiley \& Sons Inc., New York, 1990. Reprint of the 1962 original, A Wiley-Interscience Publication. 
[Sch38] I. J. Schoenberg. On the Peano curve of Lebesgue. Bull. Amer. Math. Soc., 44(8):519, 1938.

[Sch64] I. J. Schoenberg. Spline interpolation and the higher derivatives. Proc. Nat. Acad. Sci. U.S.A., 51:24-28, 1964.

[Sch83] I. J. Schoenberg. Interpolating splines as limits of polynomials. Linear Algebra Appl., 52/53:617-628, 1983.

[SNFBK10] Béla Sz.-Nagy, Ciprian Foias, Hari Bercovici, and László Kérchy. Harmonic analysis of operators on Hilbert space. Universitext. Springer, New York, enlarged edition, 2010.

[ST94] Gennady Samorodnitsky and Murad S. Taqqu. Lévy measures of infinitely divisible random vectors and Slepian inequalities. Ann. Probab., 22(4):1930-1956, 1994.

[SW53] I. J. Schoenberg and Anne Whitney. On Pólya frequence functions. III. The positivity of translation determinants with an application to the interpolation problem by spline curves. Trans. Amer. Math. Soc., 74:246-259, 1953.

[SZ07] Steve Smale and Ding-Xuan Zhou. Learning theory estimates via integral operators and their approximations. Constr. Approx., 26(2):153-172, 2007.

[SZ09] Steve Smale and Ding-Xuan Zhou. Geometry on probability spaces. Constr. Approx., 30(3):311-323, 2009.

[Tel83] Silviu Teleman. Topological properties of the boundary measures. In Studies in probability and related topics, pages 457-463. Nagard, Rome, 1983.

[Tre88] François Treves. Book Review: Pseudodifferential operators and spectral theory. Bull. Amer. Math. Soc. (N.S.), 19(1):343-346, 1988.

[Trè06] François Trèves. Basic linear partial differential equations. Dover Publications Inc., Mineola, NY, 2006. Reprint of the 1975 original.

[TW86] Lawrence E. Thomas and C. Eugene Wayne. On the stability of dense point spectrum for selfadjoint operators. J. Math. Phys., 27(1):71-75, 1986.

[vN32a] J. von Neumann. Über adjungierte Funktionaloperatoren. Ann. of Math. (2), 33(2):294-310, 1932.

[vN32b] J. von Neumann. Über einen Satz von Herrn M. H. Stone. Ann. of Math. (2), 33(3):567-573, 1932.

[vN32c] J. von Neumann. Zur Operatorenmethode in der klassischen Mechanik. Ann. of Math. (2), 33(3):587-642, 1932.

[Wid41] David Vernon Widder. The Laplace Transform. Princeton Mathematical Series, v. 6. Princeton University Press, Princeton, N. J., 1941.

[WW75] J. H. Wells and L. R. Williams. Embeddings and extensions in analysis. SpringerVerlag, New York, 1975. Ergebnisse der Mathematik und ihrer Grenzgebiete, Band 84 .

[Yos12] Kazushi Yoshitomi. Inverse scattering problems for singular rank-one perturbations of a selfadjoint operator. Asymptot. Anal., 80(3-4):213-221, 2012. 